\title{
Insulator Aging Tests with HVAC and HVDC Excitation using the Tracking Wheel Tester
}

\author{
Prepared by
}

\section{Beulah Sepo Limbo}

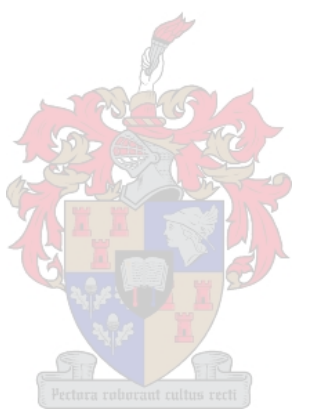

Thesis presented in partial fulfilment of the requirements for the degree

Master of Science in Engineering at the University of Stellenbosch

Supervisor: Prof H.J Vermeulen

Co-supervisor: Dr WL Vosloo

December 2009 


\section{DECLARATION}

By submitting this dissertation electronically, I declare that the entirety of the work contained therein is my own, original work, that I am the owner of the copyright thereof (unless to the extent explicitly otherwise stated) and that I have not previously in its entirety or in part submitted it for obtaining any qualification.

December 2009

Copyright (C) 2009 Stellenbosch University

All rights reserved 


\section{OPSOMMING}

Beperkte navorsingsresultate is beskikbaar oor die verouderingseffekte van oppervlakte ontladings met hoogspanning gelykstroom (HSGS) aandrywing vir praktiese isolators deur gebruik te maak van die Ontladings Wiel Toetser (OWT) metodologie. Hierdie tesis gee besonderhede van 'n eksperimentele ondersoek om die verouderingsgedrag van isolator monsters te vergelyk deur gebruik te maak van die OWT metodiek vir Hoogspanning Wisselstroom (HSWS) en positiewe en negatiewe polariteit Hoogspanning Gelykstroom (HSGS) aandrywing.

Twee reekse van toetse is gedoen. Die eerste toetsreeks het die veroudering van ses isolatorstawe, naamlik drie kamer temperatuur gevulkaniseerde silikon rubber bedekte glasmonsters en drie onbedekte glasmonsters ge-evalueer. Drie kruipafstande is gebruik vir elk van die toetsmateriale, naamlik $277 \mathrm{~mm}, 346 \mathrm{~mm}$ and $433 \mathrm{~mm}$. Die toetse is gedoen met HSWS aandrywing en die toetsmetode beskryf in die IEC 61302 standaard.

Die tweede toetsreeks het die toets van praktiese isolator monsters behels. Die toetsspanning en geleidingvermoë van die soutoplossing is ook aangepas. Ses isolators van verskillende vervaardigers, verteenwoordigend van verskillende materiale en kruipafstande, is met HSWS en positiewe en negatiewe polariteit HSGS aandrywing getoets. Die toestmonsters het uit Ethylene Propylene Diene Monomer (EPDM), Hoë Temperatuur Gevulkaniseerde Silikon Rubber (HTV SR), porselein en porselein met ' $\mathrm{n}$ Silikon Rubber (SR) bedekking bestaan. Die verouderings prestasie van die verskillende toetsmonsters vir die drie tipes aandrywing word vergelyk in terme van piek lekstroom, visuele waarnemings van die oppervlakte degradering en hidrofobiese eienskappe.

Die lekstroomdata vir HSWS aandrywing wys dat die isolators van die verskillende vervaardigers verskillend presteer, selfs vir dieselfde materiaal. Die resultate dui ook op verskille in die manier hoe die oppervlaktes degradeer, sowel as die tempo van degradering. Vir isolators wat dieselfde spesifieke kruipafstand maar verskillende materiale verteenwoordig, is gewys dat die materiaal wel die verouderingsgedrag affekteer. Vergelyking van die lekstroomdata vir die HTV SR isolators van dieselfde vervaardiger, maar met verskillende spesifieke kruipafstande, wys dat die spesifiek kruipafstand die veroudering beduidend be-invloed. 
In die algemeen, toon die toets isolators hoër piek lekstrome vir HSGS aandrywing in vergelyking met HSWS aandrywing. Die resultate vir positiewe polariteit HSGS aandrywing toon dat die droëband vonking, sowel as die ontladings, dieselfde vorm het vir al ses toets isolators. Die kleur van die droëband ontladings het gewissel van ' $n$ blouerige oranje tot donkergeel, afhangende van die intensiteit van die ontladings. Die hidrofobiese isolators, naamlik die HTV SR, EPDM and RTV SR bedekte porselein isolators, het hidrofobisiteit in die eerste week van die toetse verloor.

Die resultate vir negatiewe polariteit HSGS aandrywing toon erge oppervlakte degradering in vergelyking met die resultate vir HSWS aandrywing. Die uitwerking van positiewe polariteit HSGS aandrywing blyk egter meer beduidend te wees in terme van lekstrome en veroudering in vergelyking met negatiewe polariteit HSGS aandrywing. 


\section{SYNOPSIS}

Limited research results are available on the aging impacts of surface discharges for High Voltage Direct Current (HVDC) excitation on practical insulators using the Tracking Wheel Tester (TWT) methodology. This thesis gives details of an experimental investigation to compare the aging performance of insulator samples using the TWT for High Voltage Alternating Current (HVAC) and positive and negative polarity HVDC excitation.

Two series of tests were performed. The first series of tests evaluated the aging of six insulator rods, namely three Room Temperature Vulcanized Silicone Rubber (RTV SR) coated glass samples and three uncoated glass samples. Three creepage distances were used for each of the test materials, namely $277 \mathrm{~mm}, 346 \mathrm{~mm}$ and $433 \mathrm{~mm}$. The tests were conducted with HVAC excitation and the test methodology described in the IEC 61302 standard.

The second series of tests involved the testing of actual insulator samples. The test voltage and conductivity of the salt water solution were also adapted. Six insulators from different manufacturers, representing different materials and specific creepage distances, were tested with HVAC and positive and negative polarity HVDC excitation. The test samples consisted of Ethylene Propylene Diene Monomer (EPDM), High Temperature Vulcanized Silicone Rubber (HTV SR), porcelain and SR coated porcelain insulators. The aging performance of the different test samples for the three types of excitation are compared in terms of peak leakage current, visual observations of surface degradation and hydrophobicity properties.

The leakage current data for HVAC excitation shows that the insulators from the different manufacturers perform differently, even for the same type of material. The results also indicate differences in the way the surfaces degrade, as well as the rate of degradation. For insulators representing the same specific creepage distance, but different materials, it has been shown that the material does influence the aging performance. Comparison of the leakage current data for the HTV SR insulators from the same manufacturer, but with different specific creepage distances, shows that the specific creepage distance affect the aging performance significantly.

In general, the test insulators showed higher peak leakage currents with HVDC excitation compared to HVAC excitation. The results for positive polarity HVDC 
excitation show that the dry band arcing, as well as the discharges, has the same form for all six insulators. The colour of the dry band discharges ranged from a blue-ish orange to a dark yellow, depending on the intensity of the leakage currents. The hydrophobic insulators, namely the HTV SR, EPDM and RTV SR coated porcelain insulators, had lost hydrophobicity within the first week of testing.

The results for negative polarity HVDC excitation show severe surface degradation compared to the results for HVAC excitation. The effect of positive polarity HVDC excitation, however, seems to be more severe in terms of leakage currents and aging compared to negative polarity HVDC excitation. 


\section{ACKNOWLEDGEMENTS}

I would like to express my sincere gratitude and appreciation to the following people for their unwavering support towards the completion of the project:

- $\quad$ Firstly, I would like to thank my study leader Prof. H.J. Vermeulen for his guidance throughout the project. I would like to thank him for the constant support he has given me.

- Secondly, I would like to thank Dr Wallace Vosloo, for the expertise he shared throughout the duration of the project.

- Thirdly, I would like to thank Mr Petrus Pieterse for the technical knowledge and constant technical support he provided for the project.

- I would also like to thank the Nampower team for the financial backing of the project, in particular Mr Frank Engelbrecht, for the support he gave towards the project.

- I would also like to thank my parents, Mr. and Mrs. P.M Limbo, and the entire family for the constant support that they gave. I would not have made it thus far if they didn't believe in me.

- I would also like to thank Tafadzwa Makuza for the emotional support he gave while the thesis was being written up.

- I would also like to thank my friends for the faith they had in me.

- $\quad$ Finally, and most importantly I would like to thank god for giving me strength when I felt weak, for guiding me when I was losing my direction, and for giving me wisdom to know how to handle my project. 


\section{TABLE OF CONTENTS}

1. Project Motivation and Project Description....................................................... 18

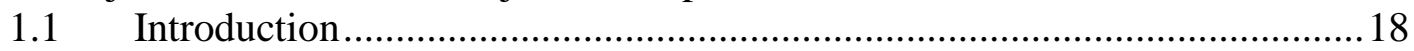

1.2 Project motivation and problem statement ……………………………....20

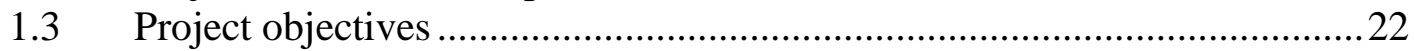

$1.4 \quad$ Scope and limitations of the investigation ...................................................22

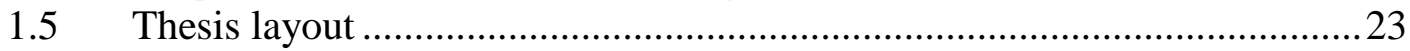

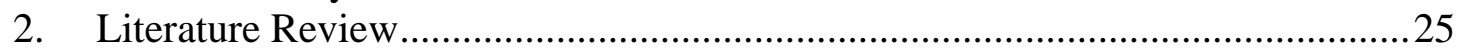

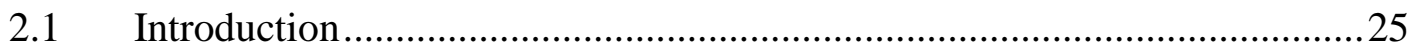

2.2 Insulator design and construction .........................................................2

2.2.1 Insulator construction and materials ...................................................2

2.2.2 Physical shape and dimensions ...........................................................26

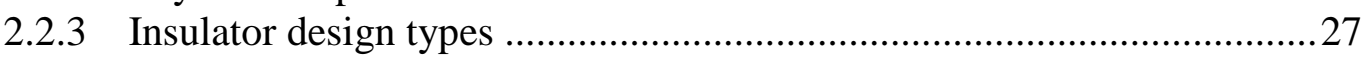

2.3 Operational performance of insulators......................................................2

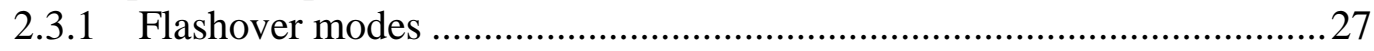

2.3.1.1 Power frequency flashover .........................................................28

2.3.1.2 Lightning and switching impulse flashover .....................................28

2.3.2 Electrical factors affecting insulators...................................................29

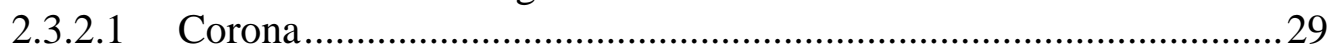

2.3.2.2 Resistance to power arc damage ......................................................29

2.3.2.3 Instantaneous or rapid conductive fog flashover ..............................29

2.3.2.4 Bird streamer flashover ..................................................................

2.3.2.5 Voltage transfer............................................................................. 30

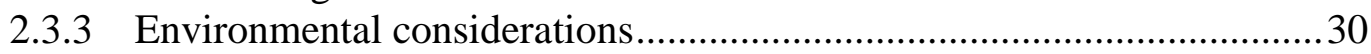

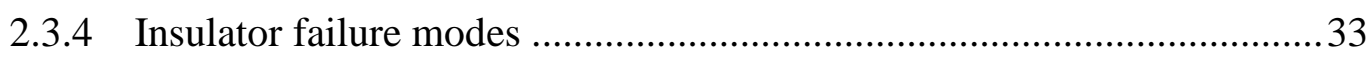

$2.4 \quad$ Insulator pollution...................................................................................

2.4.1 Pollution sources and deposits .................................................................. 34

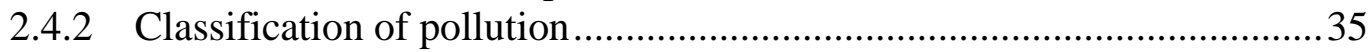

2.4.2.1 Pre-deposited pollution …………………………….......................... 35

2.4.3 Pollution conductivity ………………………........................................

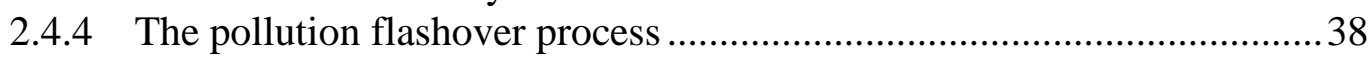

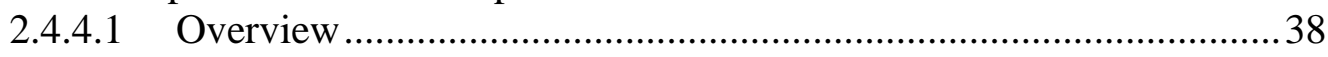

2.4.4.2 Relationships between leakage current amplitude and surface resistance 39

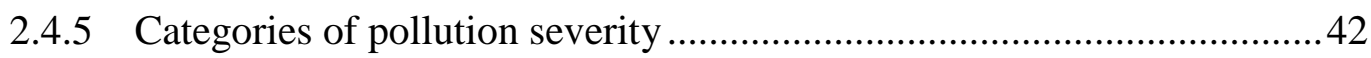

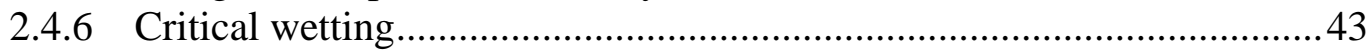

2.4.7 Pollution site severity assessment methodologies .....................................44

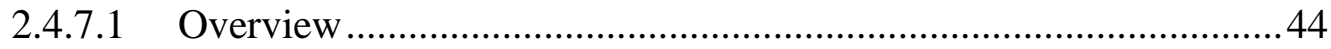

2.4.7.2 Surface pollution deposit technique..................................................44

2.4.7.3 Directional dust deposit gauge technique ...........................................45

2.4.7.4 Site severity class ...........................................................................46

2.4.7.5 Natural pollution test stations .........................................................47

3. Design of the Tracking Wheel Tester …………….........................................49

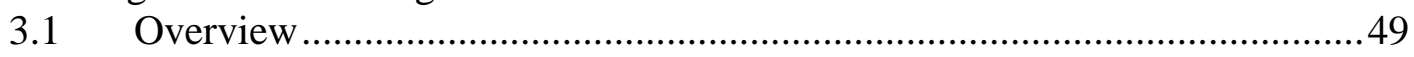

3.2 Design of the Tracking Wheel Tester ............................................................50

3.2.1 Mechanical structure …………………………....................................

3.2.2 Design of the electrical systems ............................................................51

3.2.2.1 Overview ……………………………………….......................

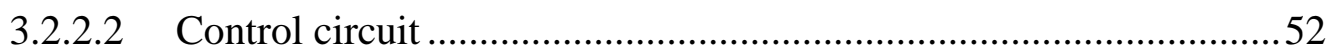




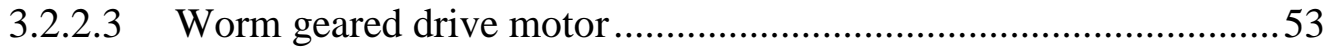

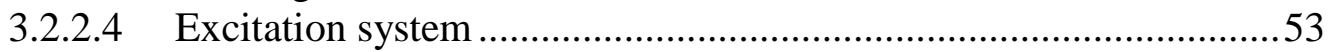

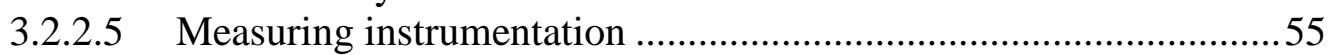

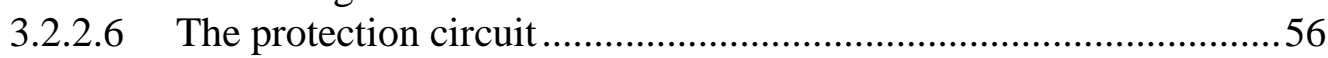

4. Summary of Test Procedures and Results .......................................................6 60

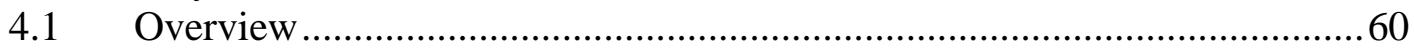

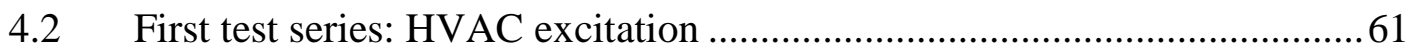

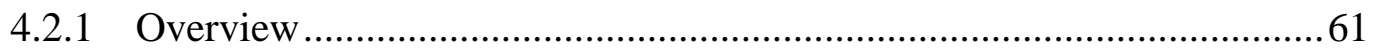

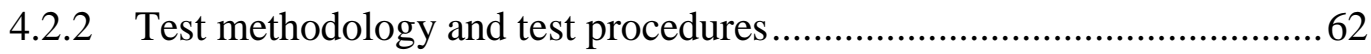

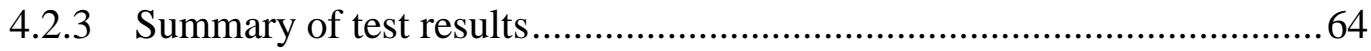

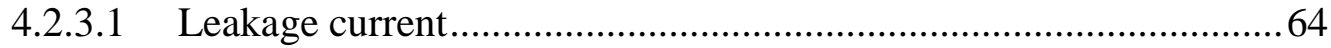

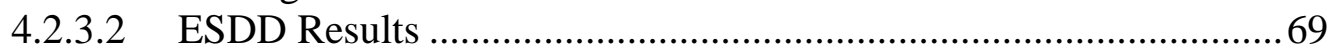

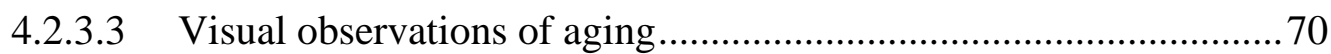

4.2.3.4 Hydrophobic properties ......................................................... 72

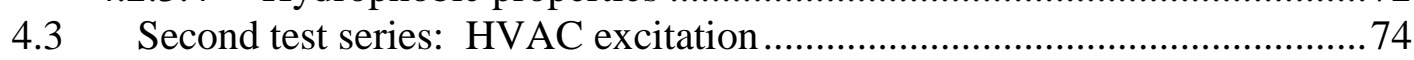

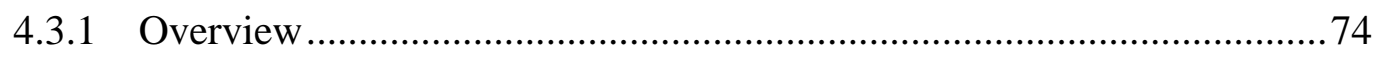

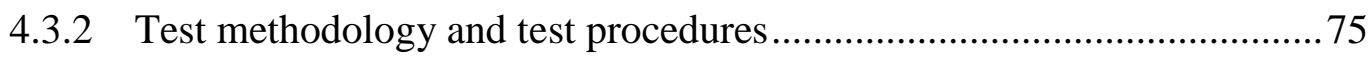

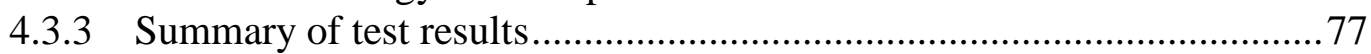

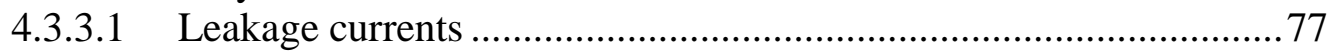

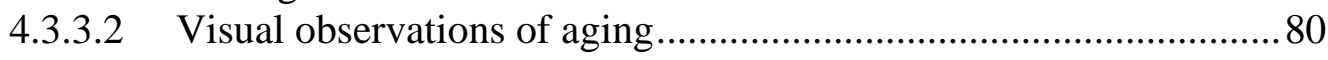

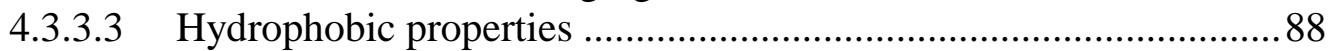

4.4 Second test series: Positive polarity HVDC excitation.............................89

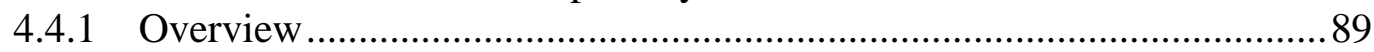

4.4.2 Test methodology and test procedures............................................... 90

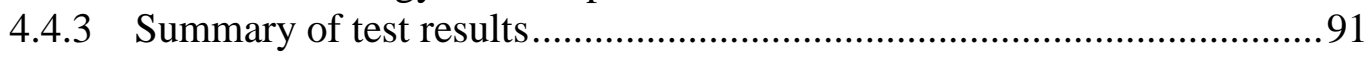

4.4.3.1 Leakage current .................................................................. 91

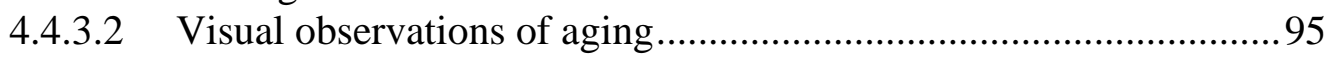

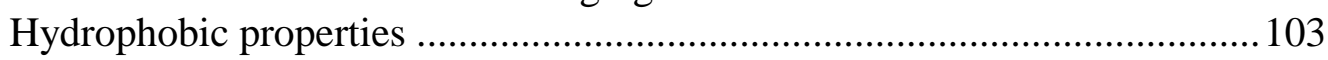

4.5 Second test series: Negative polarity HVDC excitation ........................... 105

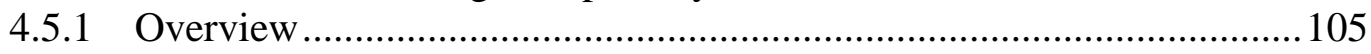

4.5.2 Test methodology and test procedures................................................. 106

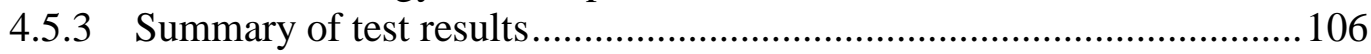

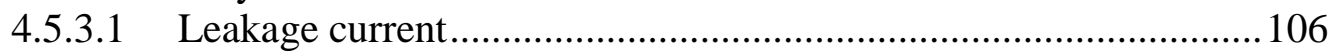

4.5.3.2 Visual observations of aging .................................................. 110

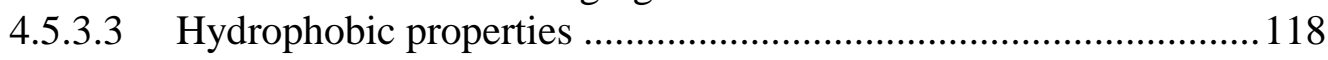

5. Comparison of Results for Different Excitation Types .................................. 121

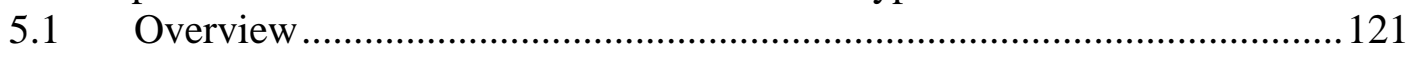

5.1.1 Results obtained for the EPDM insulator (channel 1) with a unified specific creepage distance of $28 \mathrm{~mm} / \mathrm{kV}$ from manufacturer A ...................... 121

5.1.2 Results obtained for the HTV SR insulator (channel 2) with a unified specific creepage distance of $28 \mathrm{~mm} / \mathrm{kV}$ from manufacturer A ....................... 124

5.1.3 Results obtained for the HTV SR insulator (channel 3) with a unified specific creepage distance of $28 \mathrm{~mm} / \mathrm{kV}$ from manufacturer B ...................... 127

5.1.4 Results obtained for the HTV SR insulator (channel 4) with a unified specific creepage distance of $35 \mathrm{~mm} / \mathrm{kV}$ from manufacturer $\mathrm{B}$...................... 130

5.1.5 Results obtained for the porcelain insulator....................................... 133

5.1.6 Results obtained for the RTV SR coated porcelain insulator ................ 136

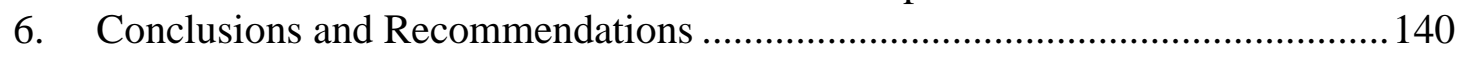

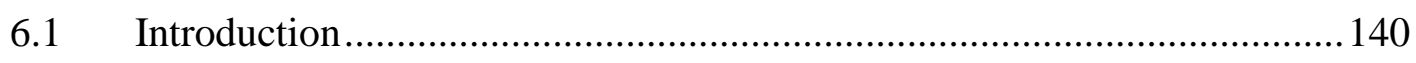


6.2 Conclusions and recommendations for test series 1 with HVAC excitation 140

6.3 Conclusions and recommendations for test series 2 with HVAC excitation 141

6.4 Conclusions and recommendations for test series 2 with positive polarity

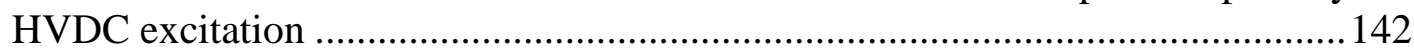

6.5 Conclusions and recommendations for test series 2 with negative polarity

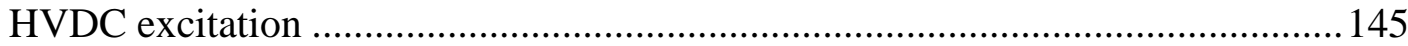

6.6 Conclusions and recommendations based on comparison between the results for different excitation voltages ......................................................................... 148 


\section{LIST OF FIGURES}

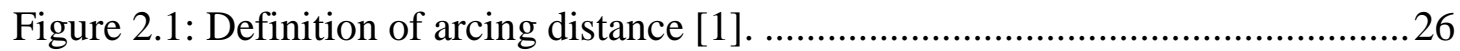

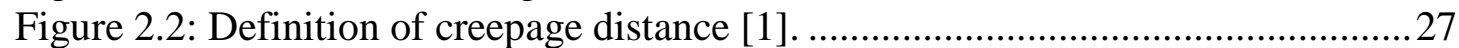

Figure 2.3: Tracking and Erosion of EPDM and SR housings ..................................33

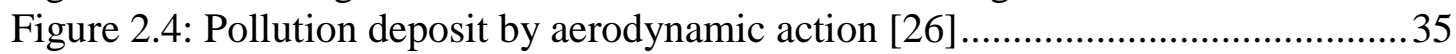

Figure 2.5: Photographs defining wettability classes WC1 to WC6 [23]....................... 37

Figure 2.6: DDDG design details: (a) As installed, (b) Dimensions ...........................46

Figure 3.1: Operating sequence of the Tracking Wheel Tester ..................................50

Figure 3.2: The Tracking Wheel Tester (TWT) ...................................................... 51

Figure 3.3: Schematic diagram of the Tracking Wheel Tester for the AC system .......52

Figure 3.4: Circuit topology of the HVDC Source ...................................................5

Figure 3.5: The HVDC source with the rectifier and capacitor, as well as part of the

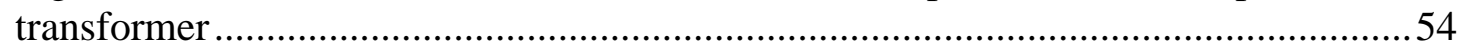

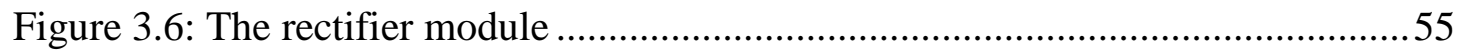

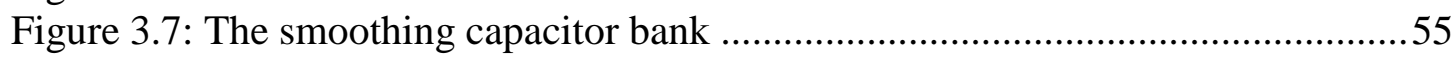

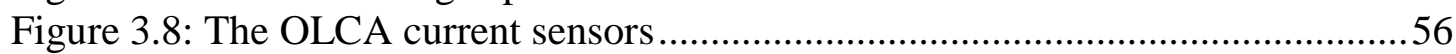

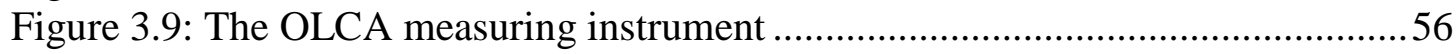

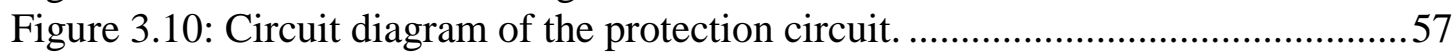

Figure 3.11: Block diagram for the protection circuit. ............................................5

Figure 3.12: Operation of the window comparator .................................................59

Figure 4.1: The six insulator samples tested during the second series of tests with

HVAC, positive polarity HVDC and negative polarity HVDC .................................61

Figure 4.2: The tracking wheel tester with the test samples used in the first series of

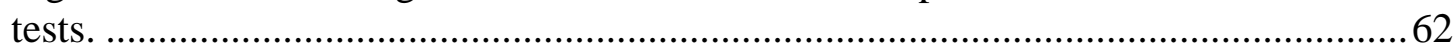

Figure 4.3: Leakage current [mA] waveform before and after the voltage is applied.64

Figure 4.4: Leakage current [mA] waveform during dry band formation ....................65

Figure 4.5: Leakage current [mA] waveform toward the end of the excitation phase 65

Figure 4.6: Peak leakage currents for the RTV SR coated glass samples ...................66

Figure 4.7: Peak leakage currents for the glass samples............................................67

Figure 4.8: Peak leakage currents for the glass and RTV SR coated glass rods with a

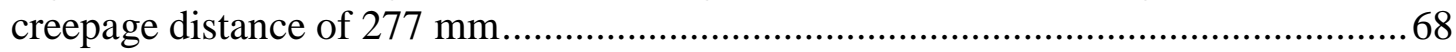

Figure 4.9: Peak leakage currents for the glass and RTV SR coated glass rods with a

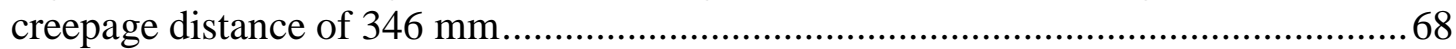

Figure 4.10: Peak leakage currents for the glass and RTV SR coated glass rods with a

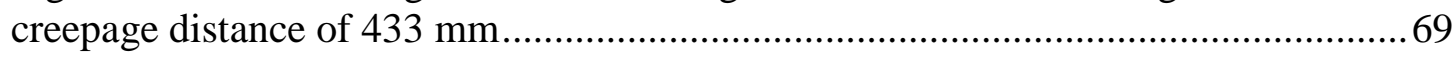

Figure 4.11: Visual aging of the glass samples...................................................... 71

Figure 4.12: Visual aging of the RTV SR coated glass samples ............................... 71

Figure 4.13: Visual aging of the RTV SR coated glass insulator with a creepage

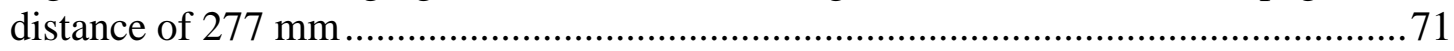

Figure 4.14: Visual aging of the RTV SR coated glass insulator with creepage

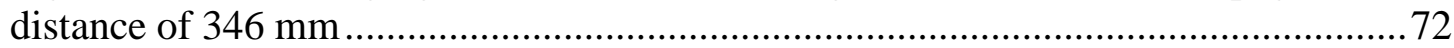

Figure 4.15: Visual aging of the RTV SR coated glass insulator with creepage

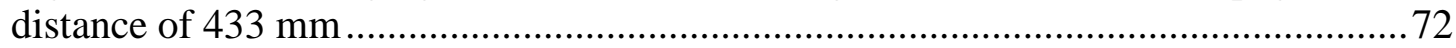

Figure 4.16: Wettability of the RTV SR coated glass sample with creepage distance of

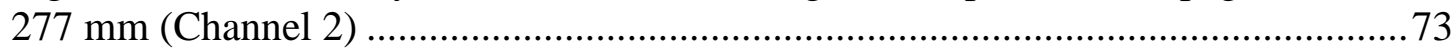

Figure 4.17: Wettability of the RTV SR coated glass sample with creepage distance of

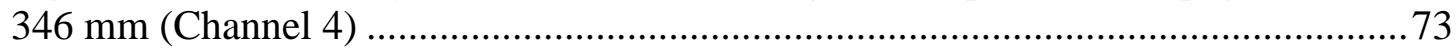

Figure 4.18: Wettability of the RTV SR coated glass sample with creepage distance of $433 \mathrm{~mm}$ (Channel 6) 
Figure 4.19: The Tracking Wheel Tester with the test samples used in the second series of tests with HVAC excitation.

Figure 4.20: Peak leakage currents for the EPDM and HTV SR insulators for HVAC

excitation

Figure 4.21: Peak leakage currents for the two HTV SR insulators with different SCD

for HVAC excitation

Figure 4.22: The puncture on Channel 3 (HTV SR insulator with SCD of $28 \mathrm{~mm} / \mathrm{kV}$ )

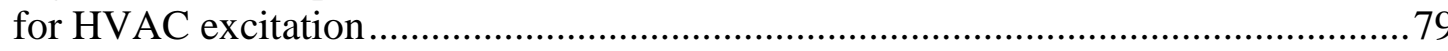

Figure 4.23: Peak leakage currents for the uncoated porcelain and RTV SR coated

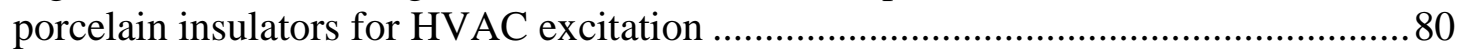

Figure 4.24: The EPDM insulator (Channel 1) before and after the test for HVAC excitation.

Figure 4.25: Detailed view of aging of the EPDM insulator (Channel 1) for HVAC excitation.

Figure 4.26: The HTV SR insulator (Channel 2) before and after the test for HVAC

excitation.

Figure 4.27: Detailed view of aging of the HTV SR insulator (Channel 2) for HVAC

excitation....

Figure 4.28 Comparison of aging of the EPDM and HTV SR insulators for HVAC excitation....

Figure 4.29: HTV SR insulator (Channel 3) before and after the test for HVAC excitation.

Figure 4.30: Detailed view of aging of the HTV SR insulator (Channel 3) for HVAC

excitation.

Figure 4.31: Comparison of aging of HTV SR insulators from manufacture A and manufacturer B for HVAC excitation

Figure 4.32: HTV SR insulator (Channel 4) before and after the test for HVAC excitation.

Figure 4.33: Detailed view of aging of the HTV SR insulator (Channel 4) for HVAC excitation....

Figure 4.34: Comparison of aging of HTV SR insulators with creepage distances of $380 \mathrm{~mm}$ and $476 \mathrm{~mm}$ for HVAC excitation.

Figure 4.35: Reference porcelain insulator (Channel 5) before and after the test for HVAC excitation

Figure 4.36: Detailed view of aging of the reference porcelain insulator (Channel 5)

for HVAC excitation.

Figure 4.37: RTV SR coated porcelain insulator (Channel 6) before and after the test

for HVAC excitation.

Figure 4.38: Detailed view of aging of the RTV SR coated porcelain (Channel 6)

insulator for HVAC excitation.

Figure 4.39: Comparison of aging of the porcelain and RTV SR coated porcelain insulators for HVAC excitation

Figure 4.40: Wettability of the EPDM insulator (Channel 1) and the HTV SR insulator (Channel 2), both with a unified SCD of $28 \mathrm{~mm} / \mathrm{kV}$

Figure 4.41: Wettability of the HTV SR insulator with a unified SCDof $28 \mathrm{~mm} / \mathrm{kV}$

(Channel 3) and the HTV SR insulator with a unified SCD of $35 \mathrm{~mm} / \mathrm{kV}$ (Channel 4)

Figure 4.42: Wettability of the reference porcelain insulator (Channel 5) and the RTV

SR coated porcelain insulator (Channel 6) 
Figure 4.43: Peak leakage currents for the EPDM (Channel 1) and HTV SR (Channel

2) insulators for positive polarity HVDC excitation.

Figure 4.44: Peak leakage currents for HTV SR insulators from manufacturer A

(Channel 2) and manufacturer B (Channel 3) for positive polarity HVDC excitation 93

Figure 4.45: Peak leakage currents for the HTV SR insulator with SCD of $28 \mathrm{~mm} / \mathrm{kV}$

(Channel 3) and the HTV SR insulator with SCD of $35 \mathrm{~mm} / \mathrm{kV}$ (Channel 4) forh

positive polarity HVDC excitation

Figure 4.46: Peak leakage currents for Channel 5 (porcelain) and Channel 6 (RTV SR

coated porcelain) for positive polarity HVDC excitation .95

Figure 4.47: The EPDM insulator with a unified SCD of $28 \mathrm{~mm} / \mathrm{kV}$ (Channel 1)

before and after the test with positive polarity HVDC excitation .....

.96

Figure 4.48: Detailed view of aging of the EPDM insulator (Channel 1) for positive

polarity HVDC excitation

.96

Figure 4.49: The HTV SR insulator with a unified SCD of $28 \mathrm{~mm} / \mathrm{kV}$ from

manufacturer A (Channel 2) before and after the test with positive polarity HVDC

excitation.

Figure 4.50: Detailed view of aging of the HTV SR insulator (Channel 2) for positive

polarity HVDC excitation

Figure 4.51: Comparison of aging of EPDM and HTV SR insulators for positive polarity HVDC excitation.

Figure 4.52: The HTV SR insulator with a unified SCD of $28 \mathrm{~mm} / \mathrm{kV}$ from

manufacturer B (Channel 3) before and after the test with positive polarity HVDC excitation.....

Figure 4.53: Detailed view of aging of the HTV SR insulator (Channel 3) for positive

polarity HVDC excitation ....

Figure 4.54: Comparison of aging of the HTV SR insulators from manufacturer A and manufacturer B for positive polarity HVDC excitation.

Figure 4.55: The HTV SR insulator with a unified SCD of $35 \mathrm{~mm} / \mathrm{kV}$ (Channel 4)

before and after the test with positive polarity HVDC excitation 100

Figure 4.56: Detailed view of aging of the HTV SR insulator (Channel 4) for positive

polarity HVDC excitation

Figure 4.57: Comparison of aging of the HTV SR insulators with unified SCD of 28 $\mathrm{mm} / \mathrm{kV}$ and $35 \mathrm{~mm} / \mathrm{kV}$ for positive polarity HVDC excitation

Figure 4.58: The porcelain insulator (Channel 5) before and after the test with positive polarity HVDC excitation

Figure 4.59: Detailed view of aging of the porcelain insulator (Channel 5) for positive polarity HVDC excitation. 102

Figure 4.60: The RTV SR coated porcelain insulator (Channel 6) before and after the test with positive polarity HVDC excitation. 102

Figure 4.61: Detailed view of aging of the RTV SR coated porcelain insulator

(Channel 6) for positive polarity HVDC excitation. 103

Figure 4.62: Comparison of aging of the porcelain and RTV SR coated porcelain for positive polarity HVDC excitation

Figure 4.63: Wettability of the EPDM insulator (Channel 1) and the HTV SR insulator (Channel 2), both with a unified SCD of $28 \mathrm{~mm} / \mathrm{kV}$...

Figure 4.64: Wettability of the HTV SR insulators from manufacturer A (Channel 2)

and manufacturer B (Channel 3), both with a unified SCD of $28 \mathrm{~mm} / \mathrm{kV}$....

Figure 4.65: Wettability of the HTV SR insulator with a unified SCD of $28 \mathrm{~mm} / \mathrm{kV}$ (Channel 3) and the HTV SR insulator with a unified SCD of $35 \mathrm{~mm} / \mathrm{kV}$ (Channel 4) 
Figure 4.66: Wettability of the porcelain insulator (Channel 5) and the RTV SR coated porcelain insulator (Channel 6)

Figure 4.67: Peak leakage currents for the EPDM insulator (Channel 1) and HTV SR insulator (Channel 2) for negative HVDC negative excitation

Figure 4.68: Peak leakage currents for the HTV SR insulators from manufacturer A (Channel 2) and from manufacturer B (Channel 3) for negative HVDC negative excitation....

Figure 4.69: Peak leakage currents for the HTV SR insulators with a unified SCDof $28 \mathrm{~mm} / \mathrm{kV}$ (Channel 3) and a unified SCDof $35 \mathrm{~mm} / \mathrm{kV}$ (Channel 4) for negative polarity HVDC excitation

Figure 4.70: Peak leakage currents for the porcelain insulator (Channel 5) and the RTV SR coated porcelain insulator (Channel 6) for negative polarity HVDC excitation....

Figure 4.71: The EPDM insulator with a unified SCD of $28 \mathrm{~mm} / \mathrm{kV}$ (Channel 1)

before and after the test with negative polarity HVDC excitation.

Figure 4.72: Detailed view of aging of the EPDM insulator (Channel 1) for negative

polarity HVDC excitation.

Figure 4.73: The HTV SR insulator with a unified SCD of $28 \mathrm{~mm} / \mathrm{kV}$ (Channel 2)

before and after the test with negative polarity HVDC excitation.

Figure 4.74: Detailed view of aging of the HTV SR insulator (Channel 2) for negative polarity HVDC excitation

Figure 4.75: Channel 1 and Channel 2 after the test with negative polarity HVDC excitation.

Figure 4.76: The HTV SR insulator with a unified SCD of $28 \mathrm{~mm} / \mathrm{kV}$ from manufacturer B (Channel 3) before and after the test with negative polarity HVDC excitation.

Figure 4.77: Detailed view of aging of the HTV SR insulator from manufacturer B

(Channel 3) for negative polarity HVDC excitation

Figure 4.78: Channel 2 and Channel 3 after the test with negative polarity HVDC excitation.

Figure 4.79: The HTV SR insulator with a unified SCD of $35 \mathrm{~mm} / \mathrm{kV}$ from manufacturer B (Channel 4) before and after the test with negative polarity HVDC excitation

Figure 4.80: Detailed view of aging of the HTV SR insulator with a SCD of 35 $\mathrm{mm} / \mathrm{kV}$ for negative polarity HVDC excitation.

Figure 4.81: Channel 3 and Channel 4 after the test with negative polarity HVDC

excitation.

Figure 4.82: The porcelain insulator (Channel 5) before and after the test with negative polarity HVDC excitation

Figure 4.83: Detailed view of aging of the porcelain insulator for negative polarity

HVDC excitation

Figure 4.84: The RTV SR coated porcelain insulator (Channel 6) before and after the test with negative polarity HVDC excitation.

Figure 4.85: Detailed view of aging of the RTV coated porcelain insulator for negative polarity HVDC excitation

Figure 4.86: Channel 5 and Channel 6 after the test with negative polarity HVDC

excitation.

Figure 4.87: Wettability of the EPDM insulator (Channel 1) and the HTV SR insulator (Channel 2), both with a unified $\mathrm{S}_{C D}$ of $28 \mathrm{~mm} / \mathrm{kV}$ 
Figure 4.88: Wettability of the HTV SR insulators with a unified SCD of $28 \mathrm{~mm} / \mathrm{kV}$ from manufacturer A (Channel 2) and from manufacturer B (Channel 3) ................119 Figure 4.89: Wettability of the HTV SR insulators with a unified SCD of $28 \mathrm{~mm} / \mathrm{kV}$ (Channel 3) and a unified SCD of $35 \mathrm{~mm} / \mathrm{kV}$ (Channel 4)

Figure 4.90: Wettability of porcelain insulator (Channel 5) and the RTV SR coated porcelain insulator (Channel 6)....

Figure 5.1: Comparison of material aging of the EPDM insulator for HVAC and positive and negative polarity HVDC excitation

Figure 5.2: Peak currents for the EPDM insulator (channel 1) for HVAC excitation

Figure 5.3: Peak currents for the EPDM insulator (channel 1) for positive polarityHVDC excitation.

Figure 5.4: Peak currents for the EPDM insulator (channel 1) for negative HVDC excitation

Figure 5.5: Comparison of hydrophobicity for the EPDM insulator (channel 1) for HVAC and positive and negative polarity HVDC excitation.

Figure 5.6: Comparison of aging for the HTV SR insulator (channel 2) for HVAC and positive and negative polarity HVDC excitation

Figure 5.7: Peak currents for the HTV SR insulator (channel 2) for HVAC excitation

Figure 5.8: Peak currents for the HTV SR insulator (channel 2) for positive polarity HVDC excitation

Figure 5.9: Peak currents for the HTV SR insulator (channel 2) for negative polarity

HVDC excitation

Figure 5.10: Comparison of the the hydrophobicity of the HTV SR insulator (channel

2) for HVAC and positive and negative polarity HVDC excitation....

Figure 5.11: Comparison of aging for the HTV SR insulator (channel 3) with a unified SCD of $28 \mathrm{~mm} / \mathrm{kV}$ for HVAC and positive and negative polarity HVDC excitation

Figure 5.12: Peak currents for HTV SR insulator (channel 3) with a unified SCD of 28 $\mathrm{mm} / \mathrm{kV}$ for HVAC excitation.

Figure 5.13: Peak currents for HTV SR insulator (channel 3) with a unified SCD of 28 $\mathrm{mm} / \mathrm{kV}$ for positive polarity HVDC excitation ....................................................... 129 Figure 5.14: Peak currents for HTV SR insulator (channel 3) with a unified SCD of 28 $\mathrm{mm} / \mathrm{kV}$ for negative polarity HVDC excitation.

Figure 5.15: Comparison of hydrophobicity for the HTV SR insulator (channel 3) with a unified SCD of $28 \mathrm{~mm} / \mathrm{kV}$ for HVAC and positive and negative polarity HVDC excitation

Figure 5.16: Comparison of material aging of the HTV SR insulator (channel 4) for HVAC and positive and negative polarity HVDC excitation 130

Figure 5.17: Peak current for the HTV SR insulator (channel 4) for HVAC excitation

Figure 5.18: Peak current for the HTV SR insulator (channel 4) for positive polarity HVDC excitation

Figure 5.19: Peak current for the HTV SR insulator (channel 4) for negative HVDC excitation.

Figure 5.20: Comparison of hydrophobicity for the HTV SR insulator (channel 4) with a unified SCD of $35 \mathrm{~mm} / \mathrm{kV}$ for HVAC and positive and negative polarity HVDC excitation 
Figure 5.21: Comparison of material aging of the Porcelain insulator (channel 5) for HVAC and positive and negative polarity HVDC excitation

Figure 5.22: Peak current for the porcelain insulator (channel 5) for HVAC excitation

Figure 5.23: Peak current for the porcelain insulator (channel 5) for positive polarity

HVDC excitation 135

Figure 5.24: Peak current for the porcelain insulator (channel 5) for negative polarity

HVDC excitation

Figure 5.25: Comparison of hydrophobicity for the porcelain insulator (channel 5)

with a unified SCD of $28 \mathrm{~mm} / \mathrm{kV}$ for HVAC and positive and negative polarity

HVDC excitation

Figure 5.26: Comparison of material aging of the RTV SR coated porcelain insulator (channel 6) for HVAC and positive and negative polarity HVDC excitation

Figure 5.27: Peak current for the RTV SR coated porcelain insulator (channel 6) for

HVAC excitation

Figure 5.28: Peak current for the RTV SR coated porcelain insulator (channel 6) for

positive polarity HVDC excitation 138

Figure 5.29: Peak current for the RTV SR coated porcelain insulator (channel 6) for negative polarity HVDC excitation

Figure 5.30: Comparison of hydrophobicity for the RTV SR coated porcelain insulator (channel 6) with a unified SCD of $28 \mathrm{~mm} / \mathrm{kV}$ for HVAC and positive and negative polarity HVDC excitation 


\section{LIST OF TABLES}

Table 2.1: Relationships between pollution level categories and environment [23] ...42 Table 2.2: Recommended Specific Creepage Distance for various pollution severity

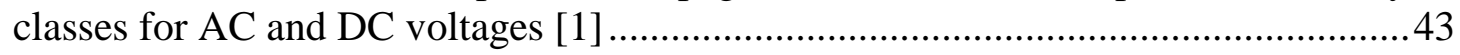

Table 2.3: The relationship between surface deposit index and severity class............47

Table 2.4: The relationship between DDG pollution index and severity class [1] ......47

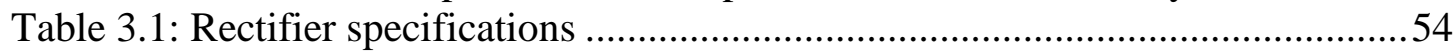

Table 4.1: Description of the samples tested in test series 1....................................60

Table 4.2: Description of the samples tested in test series 2. ..................................61

Table 4.3: The relationship between volume conductivity and ESDD value ...............70

Table 4.4: Summary of test samples for the second series of tests with HVAC

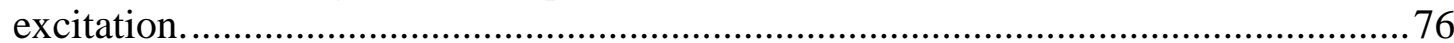

Table 4.5: Peak leakage currents for Channel 1 and Channel 2 for HVAC excitation 78 Table 4.6: Peak leakage currents for Channel 3 and Channel 4 for HVAC excitation 79 Table 4.7: Peak leakage currents for Channel 5 and Channel 6 for HVAC excitation 80 Table 4.8: Peak leakage currents for Channel 1 and Channel 2 for positive polarity

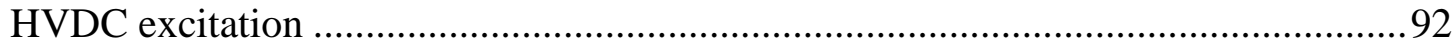

Table 4.9: Peak leakage currents for Channel 1 and Channel 2 for positive polarity

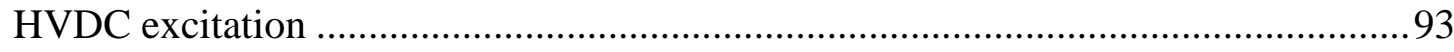

Table 4.10: Peak leakage currents for Channel 3 and Channel 4 for positive polarity

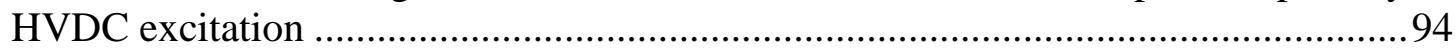

Table 4.11: Peak leakage currents for Channel 5 and Channel 6 for positive polarity

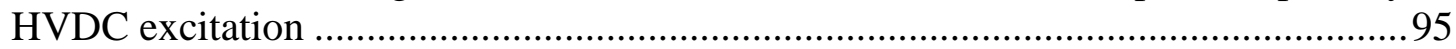

Table 4.12: Peak leakage currents for Channel 1 and channel 2 for negative polarity

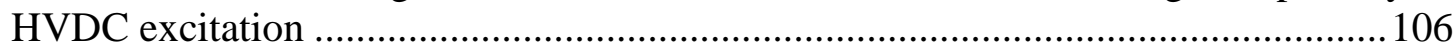

Table 4.13: Peak leakage currents for Channel 2 and Channel 3 for negative polarity

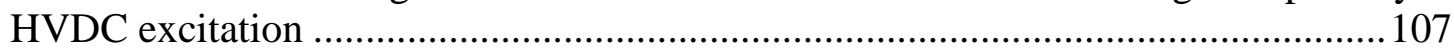
Table 4.14: Peak leakage currents for Channel 3 and Channel 4 for negative polarity

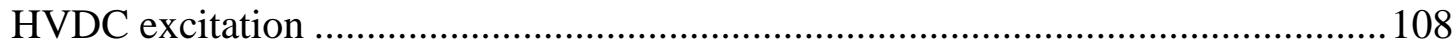
Table 4.15: Peak leakage currents for Channel 5 and Channel 6 for negative polarity HVDC excitation 109

Table 5.1: Peak currents for the EPDM insulator (channel 1) for HVAC and positive

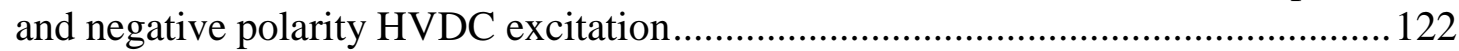
Table 5.2: Peak currents for the HTV SR insulator (channel 2) for HVAC and positive and negative polarity HVDC excitation........................................................... 125 Table 5.3: Peak currents for the HTV SR insulator (channel 3) for HVAC and positive and negative polarity HVDC excitation............................................................. 128 Table 5.4: Peak currents for the HTV SR insulator (channel 4) for HVAC and positive and negative polarity HVDC excitation. 131

Table 5.5: Peak currents for the porcelain insulator (channel 5) for HVAC and positive and negative polarity HVDC excitation Table 5.6: Peak currents for the RTV SR coated porcelain insulator (channel 6) for HVAC and positive and negative polarity HVDC excitation Table B.6.1: Table for Electro Field Simulation 


\section{LIST OF ABBREVIATIONS}

$\mathrm{AC}$

DC

EPDM

ESDD

ESP

ESCOM, Eskom

HV

HVAC

HVDC

HTV SR

IEC

KIPTS

NSDD

RTV SR

SCD

SR

TWT

USCD
Alternating Current

Direct Current

Ethylene Propylene Diene Monomer

Equivalent Salt Deposit Density

Enhanced Silicone Polymer

Electricity Supply Commission, later changed to Eskom

High Voltage

High Voltage Alternating Current

High Voltage Direct Current

High Temperature Vulcanised Silicone Rubber

International Electrotechnical Commission

Koeberg Insulator Pollution Test Site

Non-Soluble Deposit Density

Room Temperature Vulcanised Silicone Rubber

Specific Creepage Distance

Silicone Rubber

Tracking Wheel Tester

Unified Specific Creepage Distance 


\section{Project Motivation and Project Description}

\subsection{Introduction}

The first electric power system, built by Thomas Edison in September 1882, was a DC system. The excessive power loss that occurred when dealing with low voltage, however, meant that Edison's companies could only deliver energy over short distances [19]. It was soon discovered that high voltages were necessary for the effective transmission of electric power. The development of transformers and the improvement of induction motors led to an era of High Voltage Alternating Current (HVAC) transmission systems [17, 18 and 19]. HVAC transmission is preferred for the following reasons [17, 18 and 19]:

- The invention of transformers allowed power to be transmitted at higher voltages.

- Voltage conversion in an AC transmission system is simple.

- An AC system requires relatively little maintenance.

- A three phase synchronous generator is superior to a DC generator.

The commercialising of the fully-static mercury arc valve in 1954 led to the modern era of HVDC Transmission, which became more practical to use over long distances or where cables were required. The first HVDC transmission system commercialised in 1954 was a submarine cable between the island of Gotland and the Swedish mainland [17, 18 and 19].

HVDC transmission systems have the following advantages:

- $\quad$ HVDC has the ability to transmit large amounts of power over long distances at much lower capital costs and with much lower losses than HVAC.

- In undersea cables where high capacitance causes additional AC losses, it is better to use HVDC Transmission.

- HVDC transmission allows power transmission between unsynchronized AC distribution systems.

- HVDC reduces corona discharge compared to HVAC transmission lines of similar power. 
- Transmission line cost is reduced, since HVDC transmission requires only two conductors.

- $\quad$ HVDC transmission helps to reduce the Right of Way (ROW) requirements of wiring and pylons for a given power transmission capacity.

- HVDC transmission increases the capacity of an existing power grid in situations where additional lines are difficult or expensive to install.

- $\quad$ The Voltage Source Converter (VSC) technology used in a HVDC system allows the control of active and reactive power independently without any need for extra compensating equipment [16-20].

HVDC transmission systems, however, also have the following disadvantages:

- The static inverters that are used in HVDC transmission systems are expensive and cannot be overloaded.

- At shorter distances, the losses in the static inverters present in a HVDC system are bigger than in an HVAC power line.

- The control of a multi-terminal HVDC system is very complicated, since a multi-terminal HVDC system requires good communication between all the terminals.

- The cost of the inverters may not be offset by reductions in line construction cost.

- The difficulty of breaking DC currents results in costly DC breakers.

- The generation of harmonics, which requires AC and DC filters, adds to the cost of converter stations. [16, 19]

An HVAC transmission system can only interconnect synchronised HVAC networks that operate at the same frequency. Many areas with unsynchronised networks wish to share power. HVDC transmission lines make it possible to interconnect unsynchronised AC networks and have the possibility to control AC voltage and reactive power flow. HVDC transmission lines also offer increased stability in the transmission grid. The stability of a HVAC transmission system is improved by fast modulation of the DC transmission power, which is then used to damp power oscillations in an AC grid. These are some of the reasons why HVDC transmission 
lines are finding increasing application in modern interconnected power systems, particularly when dealing with long distances $[16,17]$.

The Namibia Power Corporation (Pty) Ltd. (Nampower), i.e. the electricity utility of the Republic of Namibia, embarked on a project known as the Caprivi Link Interconnector Project [29]. The Caprivi Link Interconnector is a $970 \mathrm{~km}$ HVDC line located between the Zambezi Substation (located near Katima Mulilo in Namibia) and Gerus substation (located between Otjiwarongo and Outjo in Namibia). The operating voltage of this bipolar line will be $\pm 350 \mathrm{kV}$ DC. Furthermore, the existing $400 \mathrm{kV}$ AC transmission system will be used together with the HVDC scheme. Two options will be considered for the HVDC system, namely:

- A $300 \mathrm{MW}$ monopolar scheme with earth and/or metallic return.

- $\quad$ An upgrade of the $300 \mathrm{MW}$ monopolar scheme capable of operating with earth and/or metallic return.

The initial $300 \mathrm{MW}$ monopolar will be built with existing components that won't have to be changed when the upgrade to the $600 \mathrm{MW}$ bipolar scheme takes place [29]. Due to this project a need arose to investigate the effects of HVDC excitation on insulator aging.

\subsection{Project motivation and problem statement}

When electricity was first utilised, it was soon realised that a component had to be designed that could insulate live conductors from ground potential. Any solid nonconductive material can insulate a few thousand volts from ground under dry conditions; but an effective insulator for wet and polluted conditions was difficult to design [1]. Before 1910, the highest operating voltages were between 50 and $66 \mathrm{kV}$. Over the years, however, there has been an increase in operating voltages. This led to insulators with high electrical stress ratings, increased mechanical load ratings and new transmission lines structures being introduced [1]. The pressure of environmental constraints has also led to existing lines being replaced by compact designs. However, the use of compact designs requires improved insulators and new insulator selection criteria [1].

Increased pollution also poses new challenges for insulator design. Higher industrial and agricultural production increases the amount of pollution imposed on insulators. 
This is the reason why environmental stress is such a critical factor when selecting the insulator [1].

Silicone Rubber (SR) insulators have a hydrophobic nature, which means that a continuous water layer is not easily formed. A continuous electrolytic film on the insulator will conduct current, which then leads to partial drying and arcing across this area. If this situation worsens, it will lead to a pollution flashover. Therefore, the hydrophobic property of a silicone rubber insulator is very advantageous, especially for a high voltage insulator. The aging of a SR insulator under ac or dc excitation, however, is still not fully understood. Much is unknown about the mechanisms involved at the different stages of aging, as well as the effects of aging on the lifetime of outdoor insulators [25]. Results obtained with accelerated aging methods are broadly accepted, provided there is good correlation between the field and laboratory aging results. According to Gustavsson [25], the electrical stress and the type of voltage applied is more significant compared to the material properties. It was discovered that the amount of filler present influences the degree of erosion more than the leakage current activity [25].

The use of polymeric materials with good hydrophobicity, instead of porcelain and glass, is still not fully utilised. While the polymer aging mechanism in outdoor environments has been investigated, the factors leading to the loss and recovery of hydrophobicity are also still not well understood. Furthermore, the pollution flashover on hydrophobic surfaces still needs to be investigated further [25].

Silicone rubber insulators improve the reliability of a power supply in a harshly polluted environment. Severe erosion of silicone rubber insulators has, however, been reported in coastal locations with high levels of pollution. Gustavsson [25] discovered that the length of the samples had the greatest impact on the electrical performance of the insulators, for both ac and dc voltages. He further observed that there was a relationship between the peak leakage current data and the visual observations of the samples showing surface erosion. Only the short dc energized samples showed severe erosion. Lastly, the leakage current patterns found for the short samples were similar to that found for the long samples [25].

The aging behaviour of insulator materials for positive and negative polarity HVDC excitation is still not fully understood. This represents the motivation for the subject 
of this thesis, namely to investigate the aging of insulator materials for High Voltage Alternating Current (HVAC) and positive and negative polarity High Voltage Direct Current (HVDC) excitation using the Tracking Wheel Tester (TWT).

\subsection{Project objectives}

The objectives of this investigation are as follows:

- $\quad$ Conduct a comprehensive literature review on insulator theory.

- Conduct a literature review on HVDC transmission systems, including its advantages and disadvantages.

- Develop an understanding of the design and operating standards for the Tracking Wheel Tester (TWT).

- Perform H-spice simulations for the protection circuit of the TWT.

- Design a DC source for the TWT.

- Perform simulations on a silicone rubber insulator using the Electro finite element analysis software package.

- Conduct actual insulator aging tests using the TWT for different materials, different manufacturers' products and varying Specific Creepage Distance $\left(\mathrm{S}_{C D}\right)$ for HVAC and positive and negative polarity HVDC excitation.

- Draw conclusions and propose recommendations from the above-mentioned test results.

\subsection{Scope and limitations of the investigation}

The first series of tests that was conducted was done according to the IEC 61302 standard using the TWT. Six insulator rods were used for this experiment, namely three glass samples and three RTV SR coated glass samples. Three different specific creepage distances were used. After this set of experiments was completed, recommendations were made with the view to improve the test arrangement. There was a problem due to changing water levels as a result of evaporation. This caused the leakage current value to decrease, especially for the glass samples, with decreasing water level. The temperature, as well as the conductivity of the salt water solution, were subject to changes and therefore the experiment had to be stopped every 2 days to fill the water up and keep the conductivity value constant. 
At the beginning of the second set of experiments, it was discovered that an electrolytic cell was formed due to chemical reaction produced by the combination of the aluminium insulator end fittings, the stainless steel tank and the salt water solution. This electrode/electrolyte combination is known as Lasagna cell and caused leakage currents to be circulated through the current sensing transducers. The aluminium end fittings had to be removed and the insulators had to be fitted with stainless steel end fittings [41].

The tests needed quiet a large amount of distilled water, i.e. approximately 25 litres, per day. This required water distilling equipment to be in operation on a continuous basis, including weekends.

\subsection{Thesis layout}

Chapter 1 gives a brief overview of the advantages and disadvantages of HVDC transmission systems as well as insulator theory. This chapter then introduces the motivations for and the objectives of the investigation, which is to investigate the aging of insulator materials and insulator coatings for HVAC and positive and negative polarity HVDC excitation using the TWT.

Chapter 2 presents the literature review, in which insulator theory is reviewed. It will explain in detail why and when an insulator is needed. It will also elaborate further on the factors regarding the aging mechanisms of insulators. The mechanisms and the causes of pollution flashover will be explored, including the correlation between pollution flashover and the environment.

The design of the Tracking Wheel Tester (TWT) test arrangement is discussed in Chapter 3. This chapter focuses mainly on the mechanical and electrical design of the TWT, and the modifications that were made to the original IEC 61302 standards. This chapter also elaborate on the formation of a lasagna cell/battery before the second set of experiment was conducted. Design details are given for the protection and indicator circuit designed to detect insulator failure and trip the test arrangement in the event of an over-current.

Chapter 4 presents the result of the experiments undertaken during the course of the thesis. The following four series of experiments were conducted:

- Section 4.2 discusses the results obtained from the first series of tests. This test had six test samples, namely three made of glass and three made of RTV SR 
coated glass. Three creepage distances were used, namely $277 \mathrm{~mm}, 346 \mathrm{~mm}$, and $433 \mathrm{~mm}$. A $10 \mathrm{kV}$ r.m.s AC voltage was applied to the live side of the insulator.

- Section 4.3 discusses the results obtained from the second series of tests conducted using HVAC excitation. This test featured six insulator samples, varying in terms of construction material, manufacturer and $S_{C D}$. Leakage current data were recorded and visual observations were made. Weekly video recordings were made of the individual insulators going through a test cycle. Also, the intensity of the dry bands as well as the maximum peak currents were observed.

- Section 4.4 discusses the results obtained from the second series of tests conducted using positive polarity HVDC excitation. This test featured the same six insulator samples evaluated in the second series of tests conducted with HVAC excitation. This test included evaluating the leakage current data and the visual degradation of the samples. Video recordings were made using the Corocam camera on a weekly basis. Day to day records were kept showing the intensity of the dry band arcing.

- Section 4.5 discusses the results obtained from the second series of tests conducted with negative polarity HVDC excitation. This test featured the same six insulator samples evaluated in the second series of tests conducted with HVAC and positive polarity HVDC excitation. Video recordings, as well as the visual observations, were recorded weekly. Daily observations were made as to where the dry band arcing was and how severe it was.

Chapter 5 presents an analysis and interpretation of the results.

Chapter 6 presents the conclusions and recommendations. 


\section{Literature Review}

\subsection{Introduction}

Before 1910, the highest operating voltages used were between 50 and $66 \mathrm{kV}$. By 1960, this voltage had increased tenfold. Over the years, insulators have had to meet not only the new electrical stresses, but also the increased mechanical loads that came with the new transmission lines [1]. Distribution and transmission lines pass through different environments, with pollution ranging in severity from classification as very light to very heavy [26].

Two basic types of insulator are in use today, namely ceramic (glass and porcelain) and non-ceramic insulators ( resin and composite insulators). The ceramic insulator materials include porcelain and toughened glass. A composite insulator is made up of at least two insulating parts - i.e. a core and a housing with metal fittings attached [1, 21].

It has been proven that insulator pollution flashovers on power lines contribute towards power disruption. Insulator flashovers are caused by lightning, over-voltages due to switching, and insulator pollution [26]. Task groups within various international organizations have been given the responsibility of minimising the effects of the insulator pollution flashover process. These groups are the International Electrotechnical Commission (IEC) and the CIGRE taskforce [26].

\subsection{Insulator design and construction}

\subsubsection{Insulator construction and materials}

Insulators can be designed with a number of different materials, including porcelain, toughened glass, epoxy resin and polymer composite materials. The word composite refers to insulators with a fibreglass core, covered by a housing which protects the core from the environment and gives the required electrical characteristics. The fibreglass core provides the necessary mechanical strength [1].

There are two main types of housing material in use today namely Ethylene Propylene Diene Monomer (EPDM) and Silicone Rubber (SR). EPDM has both a high mechanical strength and tracking resistance while SR has a high resistance to ultraviolet degradation and a hydrophobic property. As will be explained in section 2.3.1, the term hydrophobic means that the surface repels water [1]. 
The term insulator shed refers to the projection from the core of an insulator. It is used to increase the creepage distance of an insulator. The shape and geometry of the sheds have a significant effect on the performance of the insulator in the field. Insulator sheds can be grouped into three main categories, namely normal, alternating and under-ribbed sheds [1].

\subsubsection{Physical shape and dimensions}

From a physical perspective, insulators are characterized by the physical dimensions that are important for the optimum selection of insulators, as these dimensions determine crucial electrical and mechanical capabilities. The main dimensions of interest are arcing distance, creepage distance and puncture distance. The arcing distance and creepage distance can be described as follows:

\section{- $\quad$ Arcing distance}

Arcing distance is defined as the shortest distance between the conducting terminals that normally have the operating voltage between them, and is typically the shortest distance in the air external to the insulator. This dimension, shown in Figure 2.1, determines the power frequency and impulse flashover voltage of a clean insulator. The arcing distance plays a big role in determining the electrical requirements of the device and defines the physical size of the insulator at a specified operating voltage [1].

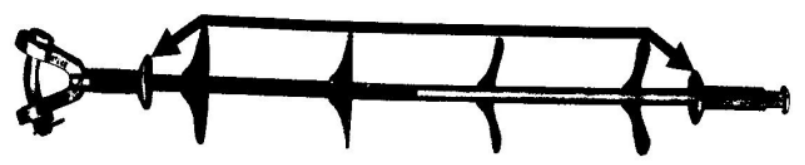

Figure 2.1: Definition of arcing distance [1].

- $\quad$ Creepage distance

Creepage distance is defined as the shortest distance along the contours of the external surfaces of the insulating parts of the insulator, between the conducting terminals that normally have the operating voltage between them. This dimension, shown in Figure 2.2, determines power frequency flashover voltage of a polluted insulator [1]. The most important factor to be taken into consideration in selecting an insulator for a specific operating voltage is the Specific Creepage Distance (SCD). Specific Creepage Distance is defined as 
the creepage distance divided by the highest phase-to-phase system voltage, thus giving it the unit $\left[\frac{m m}{k V}\right][1]$.

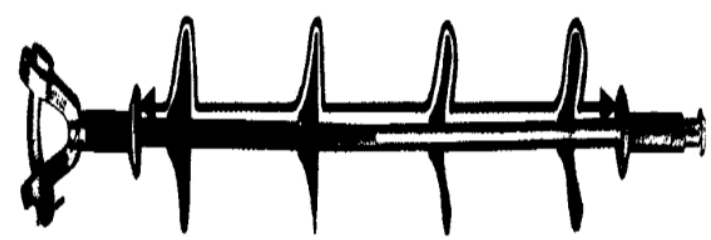

Figure 2.2: Definition of creepage distance [1].

\subsubsection{Insulator design types}

Insulator designations are typically derived from the physical design and the application of the insulator in the system. The common insulator types in use today are the Pin Insulator, Line Post Insulator, Composite Line Post Insulator, Cap-and-Pin Disc Insulator, Long Rod Insulator, Composite Long Rod Insulator, Station Post Insulator, Pedestal Post Insulator, Bushing, Apparatus Insulator, Stay wire Insulator, and the Guy Strain Insulator.

\subsection{Operational performance of insulators}

\subsubsection{Flashover modes}

Air, at atmospheric pressure, is a good insulating material. However, it has no mechanical properties capable of supporting high voltage conductors. This is the reason why high voltage insulators were developed in the nineteenth century. The focus will be on the electrical properties necessary for the effective selection of insulators as well as minimizing the probability of electrical flashovers [1].

Hydrophobicity is the ability that an insulator surface has to repel water. This is represented by water droplets occurring on the surface of the insulator. This hydrophobic characteristic is important, since the pollution flashover of external insulation involves dry band arcing which is produced as a result of heating and evaporation of liquid leakage path. This conducting electrical path is produced by the combination of the pollution layer and the liquid mixture present on the insulator surface [21]. 
When the insulator is highly hydrophobic, water that is deposited on the insulator will form water droplets. These water droplets will reduce the occurrence of leakage currents and inhibit the dry band arcing process [21].

\subsubsection{Power frequency flashover}

The insulator, under both dry and wet conditions, has to withstand the power frequency operating voltage $\left(\mathrm{U}_{n}\right)$ and overvoltage $\left(\mathrm{U}_{m}\right)$. The arcing distance has a significant effect on the dry and wet power frequency flashover voltage [1]. The power frequency flashover voltage is reduced when a conducting electrolytic pollution layer is present on the insulator surfaces. The pollution flashover performance has a huge effect on the power system reliability. Consequently, insulators should be chosen to perform adequately for a given pollution severity environment. It must also withstand the pollution condition to which it is subjected. Pollution conditions are currently ignored for lightning and switching impulse flashover levels [1].

\subsubsection{Lightning and switching impulse flashover}

The insulator has to withstand lightning and the switching impulse overvoltages, without causing permanent damage to the insulator itself. The arcing distance is the main factor determining the dry lightning and wet switching impulse flashover voltages.

Lightning impulse flashover is the main consideration for system voltages below 300 $\mathrm{kV}$, since switching impulses typically do not cause flashover at this voltage levels, while switching impulse flashover is the main consideration for system voltages of $300 \mathrm{kV}$ and above. For large air gaps, the streamer or leader breakdown mechanisms operate such that the switching impulse has more time to bridge the gap. The magnitude of the switching impulse is related to the system voltage while the magnitude of lightning impulses depends on the severity of the lightning, as well as the quality of the grounding of the towers [1]. 


\subsubsection{Electrical factors affecting insulators}

\subsubsection{Corona}

In uniform and quasi-uniform field geometries, ionization usually leads to complete breakdown of the gap. In non-uniform geometries, discharges are observed in areas of high field concentration before the breakdown occurs. These discharges can be transient or steady-state and are referred to as corona [21].

All hardware, including the insulator must have a corona inception voltage greater than the highest system voltage $\left(\mathrm{U}_{m}\right)$. Corona emits acoustic noise and radio and TV interference. Corona also generates ultraviolet radiation, ozone, and acids (in the presence of moisture). These are environmentally unacceptable and have a negative effect on polymeric insulating materials. In order to prevent corona discharges, liveend corona rings are applied for system voltages greater than $200 \mathrm{kV}$ [1].

\subsubsection{Resistance to power arc damage}

The insulator and the hardware have to withstand the power arc of the current associated with the system under flashover conditions [1]. Arcing horns or rings are used for porcelain long rod insulators to divert the arc away from the insulating material, as the thermal shock could lead to mechanical failure. Further recommendations include that the arcing horns should not be attached directly to the insulator end fittings but to the nearby hardware [1].

\subsubsection{Instantaneous or rapid conductive fog flashover}

Instantaneous pollution is when a clean insulator with a low conductive state gets contaminated with a high conductive state, and experiences flashover within a short period of time (<1hour). It then returns to a low conductive state after the event has occurred. The conductive layer is a liquid electrolyte such as salt spray, salt fog or industrial acid fog [1].

Instantaneous pollution is mostly experienced in areas that are close to the coast or nearby a chemical plant. In these areas, increasing the creepage distance or altering the profile may not be effective. Therefore, the use of insulators with hydrophobic surfaces is recommended [1]. 


\subsubsection{Bird streamer flashover}

Bird streamers are known to cause instantaneous pollution flashover. It involves a type of bird excrement that forms a continuous and highly conductive stream which causes the length of the air gap to be reduced, thus leading to flashover [1].

\subsubsection{Voltage transfer}

When an insulator is clean and dry, the highest electrical stress occurs on the live side. When the insulator becomes polluted and wetted, the highest electrical stress occurs in areas with a small radius of curvature, like the shed tips. When a dry band is formed, the highest electrical stress occurs in the dry band region. Voltage transfer takes place on the insulator and this can lead to high electric field stresses being present anywhere on the insulator surface [1].

\subsubsection{Environmental considerations}

There are a number of environment factors that affect the performance of high voltage insulators. These include the following [1]:

- $\quad$ The direction and speed of the wind, precipitation (rain, etc), relative humidity and the position of the pollution sources all determine the final pollution deposits on an insulator surface. Therefore, the environment plays a major role in insulator pollution flashover.

- Ultraviolet solar radiation can cause chalking, crazing, and cracking on the ageing of non-ceramic materials.

- The direction and speed of the wind temperature, ice and snow can influence the mechanical forces on an insulator.

- Lightning activity, soil resistivity, and bird streamers can affect the insulator flashover performance.

Weather conditions such as temperature, humidity, rain, fog, wind, solar radiation, snow and ice, lightning and air density can have significant impacts on the electrical and/or mechanical performance of HV insulators [1]. These can be summarized as follows: 


\section{- Temperature:}

When the insulator surface temperature falls below the atmospheric dew point temperature, moisture will begin to form on the insulator surface. This situation usually takes place in the early morning hours, when the temperature of the insulator surface is lower than the ambient temperature [1]. Ambient temperature also affects the insulator loading because of the influence it exerts on conductor tension [1].

- Humidity:

Relative humidity is a measurement of the moisture level in the air. A relative humidity level of more than $75 \%$ signifies that the pollution layer on an insulator could be wetted. This combination of pollution and moisture can produce a conductive electrolytic layer which gives rise to leakage currents. While high humidity does not affect the withstand voltage of an insulator directly, the wetting of the pollution layer on the insulator surface can lead to a pollution flashover [1]. The pollution on an insulator surface can also be washed away with a high enough level of humidity.

There is a relationship between electrical activity and relative humidity. Certain insulator materials can be overstressed in areas with a constant high humidity level, resulting in degradation by hydrolysis, i.e. the chemical process whereby a molecule is divided into two parts with the addition of a water molecule [1].

- Rain:

A conductive layer is formed by combining rain with a pollution layer, since rain can have the benefit of removing the pollution layer from an insulator surface. Rain in excess of $10 \mathrm{~mm} / \mathrm{h}$ can remove up to $90 \%$ of the pollution from a ceramic or glass insulator surface. Even though light rain can cause active pollution, the combination of rain and wind acts as a good natural insulator cleaner [1].

- Fog:

Fog is formed as moisture condenses on particles when the temperature of a volume of air falls below the dew point. This is either caused by the cooling of the ground (radiation fog) or warm air moving over a cooler surface (advection 
fog). A conductive fog is formed when the suspended particles become soluble and conductive, like salt fog [1]. Advective fog occurs when air flows over a cool surface. Radiation fog will be formed when the net radiation is negative, and the air is cooled below the dew point. It will gradually thicken and deepen as the night progresses [26].

Fog can cause flashover when it wets the pollution layer. If the fog is conductive, flashover will take place within a short period of time [1].

- Wind:

Wind affects the transportation and depositing of pollution and moisture on an insulator surface. There is a correlation between Salt Deposit Density (SDD) and wind. Thus, an increase in pollution deposit would mean a cubic increase in the wind speed level. The pollution layer on the insulator surface is not uniform, but depends on the shed shape. Strong winds that carry sand particles or rain can also remove pollution from the insulator surface [1].

- Solar radiation:

Solar radiation affects the ambient air mass by heating. As a result, solar radiation also affects the wind speed and direction and the relative humidity levels. Solar radiation also heats the insulator surface [1].

- Lightning:

Lightning can cause insulator flashover by striking a phase conductor, shield wire or structure causing back flashover, or induced overvoltage. The insulator must be able to withstand the naturally induced lightning impulses without puncture from flashovers [1].

- Moisture absorption:

The pollution layer of an insulator can be wetted through moisture absorption by insoluble components of the pollution layer [26]. If the vapour pressure in the atmosphere is higher than that of the solution vapour pressures, the solution will absorb moisture from the atmosphere. The vapour pressure belonging to the aqueous is always higher than the water vapour pressure. The chemical composition of the pollution determines the rate of moisture absorption on the 
insulator surface. Continuous moisture absorption will lead to surface discharges and eventually flashover [26].

\subsubsection{Insulator failure modes}

Failure of the insulator housing only affects the electrical performance of the insulators, since the housing has no mechanical function. The environmental stress, as well as the voltage being applied to the insulator may cause electrical discharges. These electrical discharges could cause erosion, and on some early housing materials, tracking. A tracked path reduces the creepage distance of the insulator, and if long enough, leads to flashover. The nature of the tracking and erosion depends on the material properties. Figure 2.3 shows examples tracking and erosion damage.
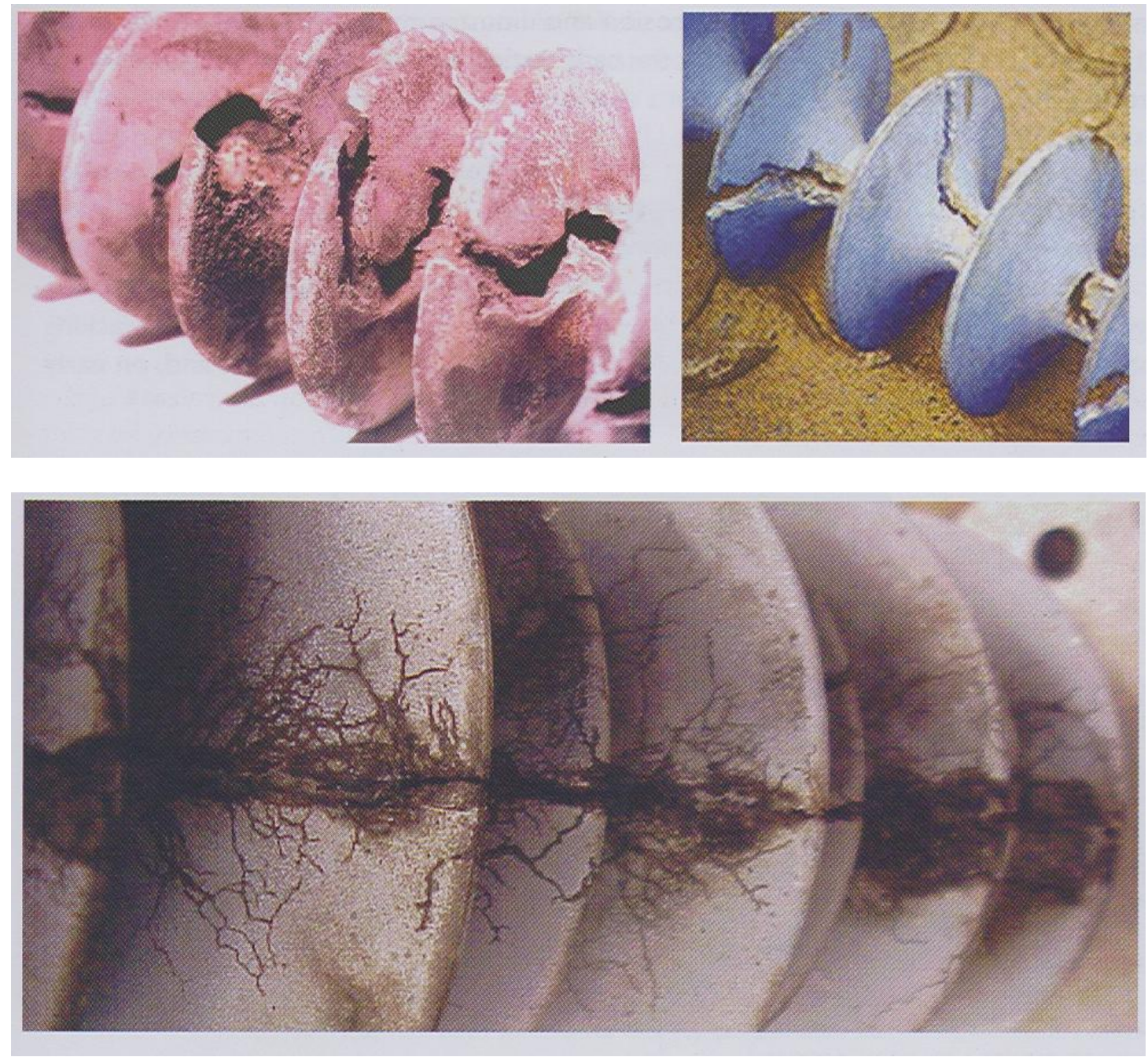

Figure 2.3: Tracking and Erosion of EPDM and SR housings 


\subsection{Insulator pollution}

\subsubsection{Pollution sources and deposits}

A pollution source is defined as a source that emits a substance into the atmosphere and the pollution experienced in an area is obtained from the effect that these emissions has on that specific area [26]. There are two main insulator pollution processes, namely pre-deposited pollution and instantaneous pollution. Pre-deposited pollution happens over time, and usually requires the pollution layer to be wetted before a conducting electrolyte is formed. Instantaneous pollution is already a conducting electrolyte [1].

The sources of pollution that contaminant the insulators are as follows [21]:

- Sea salt

- Industrial products which contain soluble salts

- $\quad$ Road salts

- $\quad$ Bird excrement

- $\quad$ Dessert sands

The process of pollution deposit on the insulators occurs in the following ways:

- Electrostatic attraction of electrically charged particles.

- Gravity.

- Aerodynamic catch of the insulator

The aerodynamic catch of the insulator, explained in Figure 2.4, is the most important process of pollution deposit on the insulator [26]. When air containing suspended particles flows around an insulator, the particles will be separated depending on how dense they are. The heavier particles, such as sand, will be deposited on the leading side, while the lighter particles, such as air, will follow the air current more closely. In other words, the lighter particles will be deposited on the side of the insulator where the curve of the air flow is sharp [26]. 


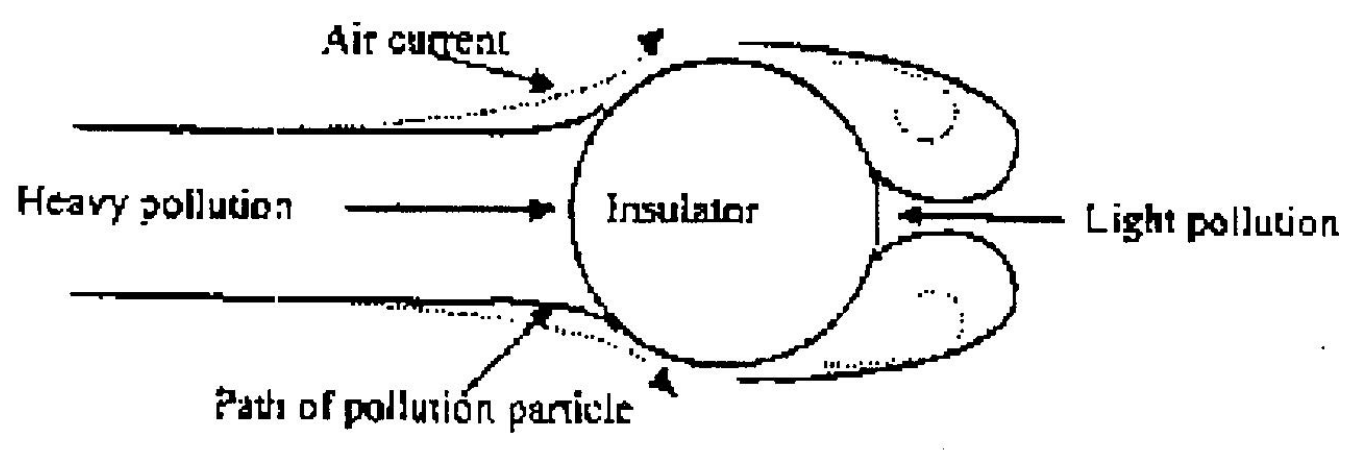

Figure 2.4: Pollution deposit by aerodynamic action [26]

\subsubsection{Classification of pollution}

\subsubsection{Pre-deposited pollution}

Pre-deposited Pollution is divided into two main groups, namely active and inert pollution. Active pollution forms a conductive layer while inert (non-soluble) pollution forms a binding layer for the conductive pollution. Inert pollution also contributes to the area available for leakage current [1]. Pre-deposited active pollution is measured in terms of conductivity, while inert pollution is measured in terms of mass [1].

Active pollution has the following examples [26]:

- $\quad$ Conductive pollution: Metallic dust

- High solubility salts: Sodium Chloride $(\mathrm{NaCl})$, Magnesium Dichloride $\left(\mathrm{MgCl}_{2}\right)$, Sodium Sulphate $\left(\mathrm{NaSO}_{4}\right)$, etc.

- $\quad$ Low solubility salts: Gypsum, fly ash and cement

Hydrophilic pollution is when the surface of the insulator absorbs water and forms a continuous conducting film. Whereas, hydrophobic pollution occurs when the surface of the insulator repels water and ends up forming water droplets [26].

Inert pollution has the following examples [26]:

- Hydrophilic pollution: Kaolin, cement, clay, $\mathrm{SiO}_{2}$, etc.

- Hydrophobic pollution: Silicone grease, oil, etc. 
A hydrophobic surface plays a very important role in limiting the flow of leakage current and preventing pollution flashover. A hydrophobic surface has low levels of wettability and thus repels water. A hydrophilic surface, to the contrary, has high levels of wettability and thus attracts water. The term wettability is defined as the ability of a surface to be wetted by a liquid $[1,23]$.

There are three methods used to determine the degree of wetness of a surface, namely the contact angle method, the surface tension method and the spray method [23]. These can be summarized as follows [23].

- $\quad$ Contact angle method:

The contact angle method involves the measurement of the angle between the edge of a single droplet of water and the surface of a solid material. When the surface is in a horizontal position, the advancing and receding contact angle is measured by adding or subtracting water from the droplet [23]. The contact angle is affected by the roughness of the surface. Therefore the contact angle measured on a polluted surface may differ from the contact angle measured on smooth, clean and planar surfaces [23].

- $\quad$ Surface tension method:

The surface tension method is best explained by the observation that droplets of a series of organic liquid mixtures, with increasing surface tension, will result in different ability to wet the insulator surface. This is an extension of IEC 60674 - $2^{1}$ used for obtaining the surface tension of polyethylene and polypropylene films. This method involves large amounts of liquids to cover a wider surface tension for both hydrophobic \& hydrophilic insulators [23].

There are restrictions when using this method for polluted insulator surfaces, since the surface tension method is affected by interaction between certain types of surface pollution [23].

- $\quad$ The spray method

The spray method involves evaluating the insulator surface by visual inspection after the insulator has been exposed to water mist for a short period of time. The wetness of the insulator surface is determined by comparing the appearance of the surface with reference material representing different wettability classes 
[23]. Figure 2.5 shows photos representing the different wettability classes, namely completely hydrophobic (wettability Class 1 ) to completely hydrophilic (wettability Class 6) [1, 23].

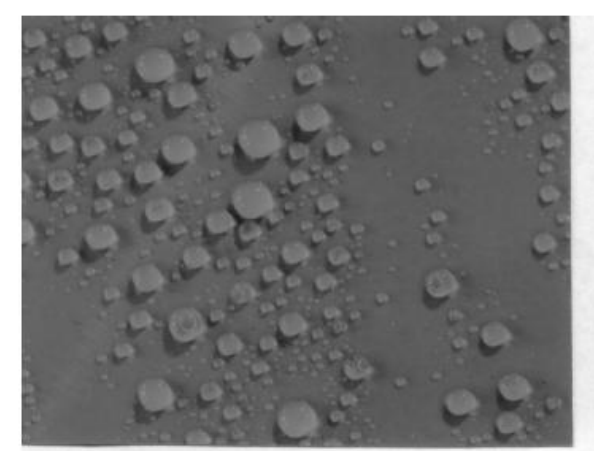

WC 1

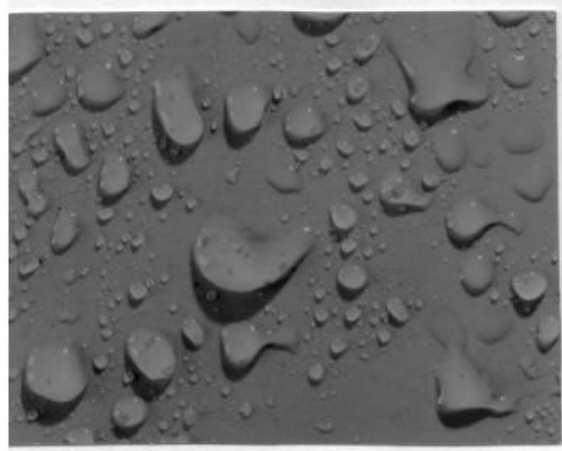

WC 3

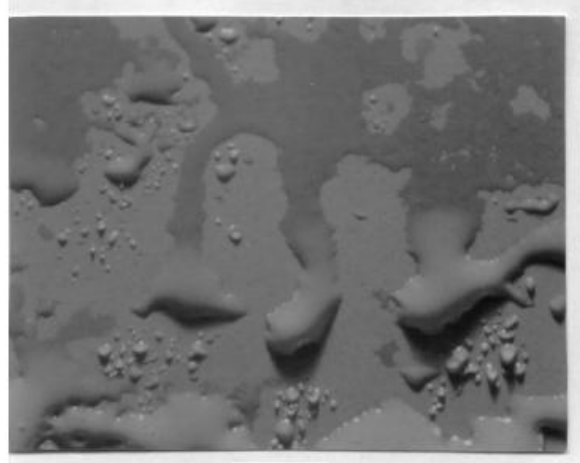

WC 5

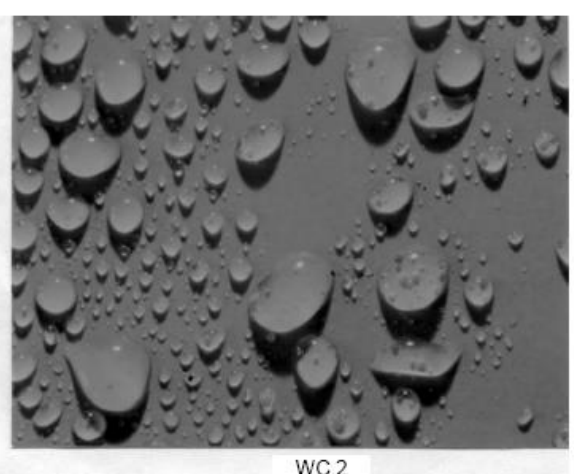

NC2 2
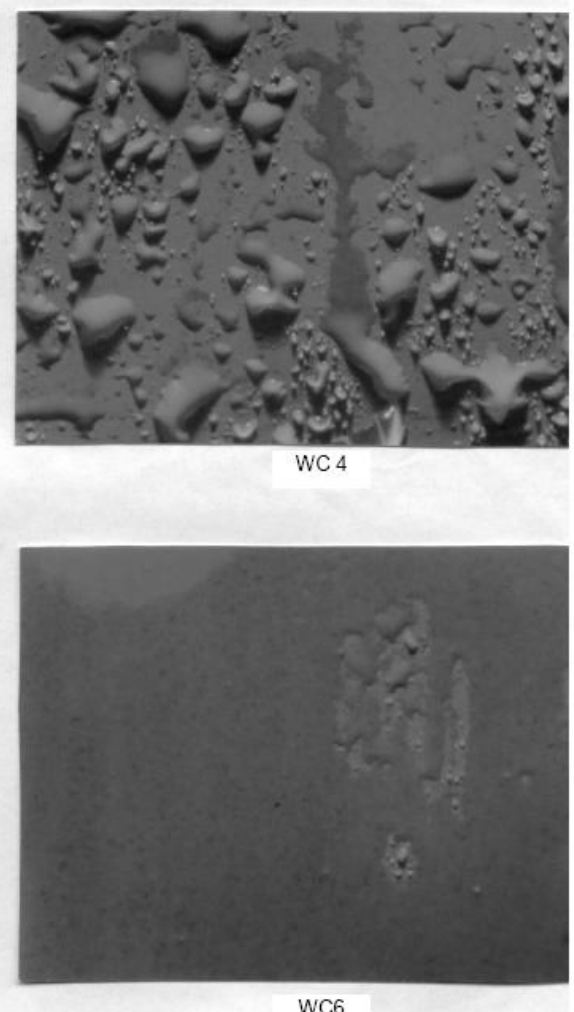

WC6

Figure 2.5: Photographs defining wettability classes WC1 to WC6 [23].

\subsubsection{Pollution conductivity}

The conductivity of the pollution layer plays a big role in determining when the insulator will flash over. A critical wetting condition occurs when the environment affects the results and a high surface conductivity is produced. Knowledge of the pollution type can help to determine the critical wetting condition for a specific area [26]. The wetting conditions for the different pollution types can be summarized as follows [26]: 
- Active pollution:

The wetting is critical for a conductive pollution. While a high solubility salt sometimes have critical wetting under a low wetting rate (e.g., mist, dew or light rain), low solubility salts have critical wetting when subject to a high wetting rate (e.g., heavy rain) [6].

- Inert pollution:

Hydrophilic pollution enhances pollution wetting while hydrophobic pollution inhibits pollution wetting [6].

\subsubsection{The pollution flashover process}

\subsubsection{Overview}

When salt or dust is deposited on the insulator surface due to the marine or industrial environment, the electrical performance of the insulator can be severely affected. This particularly true when the insulator is wetted either by rain, mist or fog, which give rise to the formation of a conducting liquid electrolyte surface layer. The conducting liquid electrolyte will allow the flow of leakage current that can lead to a pollution flashover, if large enough. Insulators must therefore be designed to cope under the most extreme environment conditions it will be exposed to [26]. The environment and local climatic conditions not only affect the pollution flashover mechanism but contribute to the ageing of insulators. A key criterion is that the pollution severity of the site has to be known [26].

The pollution flashover process for an insulator with a hydrophilic surface can be described as follows [1]:

- The insulator becomes coated with a pollution layer containing soluble salts or dilute acids or alkalis.

- If the pollution layer is initially non-conducting, the pollution layer becomes conductive when the surface of the polluted insulator is wetted either completely or partially by fog, mist, and light rain, melting snow or ice. Heavy rain can wash away and remove the electrolytic component of the pollution layer, without initiating the other stages. It can also bridge the gaps between sheds, which promotes flashover. 
If the pollution layer is a conducting liquid electrolyte, once the insulator becomes energized, a leakage current begins to flow. As a result, part of the pollution layer begins to dry.

- The drying of the pollution layer is non-uniform. This leads to a conducting pollution layer that is broken down by dry bands in some parts. These eventually interrupt the flow of leakage current.

- When the line-to-earth voltage is applied across the dry bands, air breakdown occurs and the dry bands are bridged by arcs, which are electrically in series with the resistance of the undried portion of the pollution layer. A surge of leakage current occurs each time the dry bands on an insulator spark over.

- If the resistance of the undried part of the pollution layer is low enough, the arcs bridging the dry bands are able to burn continuously, and extend along the surface of the insulator. This leads to a reduction in the resistance in series with the arcs. There is an increase in current, allowing the arc to bridge even more of the insulator surface and a flashover can result.

The leakage current associated with the dry band phenomena is in the order of $250 \mathrm{~mA}$ [21].

The pollution flashover process thus results from an interaction between the insulator pollutant, wetting conditions and applied voltage [3]. The following conditions have to be considered in investigating the insulator pollution flashover process [3]:

- Local climatic and environmental conditions surrounding the insulators.

- Pollution deposit, wetting condition and final pollution deposit on the insulators.

- Visual condition of the insulator.

- Electrical activities/discharges on the insulator.

- $\quad$ Leakage current flowing on the insulator.

\subsubsection{Relationships between leakage current amplitude and surface resistance}

Research has shown that the power-frequency flashover voltage can be reduced by a factor of up to eight if the insulator surface is lightly polluted. Leakage current is one of the parameters considered in the measurement of insulator performance. Small currents, i.e. in the order of several $\mathrm{mA}$, can cause severe damage to non-ceramic 
insulating materials. This leads to electrical and/or mechanical breakdown of the insulator.

The flashover probability becomes very high if the insulator leakage current approaches a certain threshold value. This threshold value, $I_{\max }$, is defined as the peak amplitude of the leakage current for the half cycle immediately preceding flashover $[1,26]$. The peak leakage current amplitude before flashover is given by the relationship

$$
\mathrm{I}_{\text {max }}=\left(\frac{S C D}{15.32}\right)^{2}[\mathrm{~A}]
$$

where the Specific Creepage Distance $S_{C D}$ is given by the relationship

$$
S C D=\frac{L C D}{U_{m}}\left[\frac{m m}{k V}\right]
$$

and LCD denotes the total insulator creepage distance $[\mathrm{mm}]$ and $U_{m}$ denotes the maximum system phase-to-phase rms voltage $[\mathrm{kV}]$.

The surface layer resistance is the main factor determining the magnitude of the insulator leakage current and is also used to determine whether an insulator will flash over or not [26]. The following relationship is used to determine the surface layer resistance $\mathrm{R}_{p o l}$ of a uniform electrolytic pollution layer on the insulator [1]:

$$
\mathrm{R}_{p o l}=\frac{\rho_{p o l} \cdot L C D}{A_{p o l}}[\mathrm{M} \Omega]
$$

where the parameters are defined as follows:

$$
\left.\rho_{p o l}: \text { Volume resistivity of the electrolytic pollution layer [M } \Omega . \mathrm{mm}\right]
$$

LCD: Total insulator creepage distance [mm]

$$
\begin{aligned}
& \mathrm{A}_{p o l} \text { : Cross-sectional area of the electrolytic pollution layer at position } 1 \\
& {\left[\mathrm{~mm}^{2}\right]}
\end{aligned}
$$

$\mathrm{A}_{p o l}$ is given by the relationship 


$$
\mathrm{A}_{p o l}=\pi \times \mathrm{D}(\mathrm{l}) \times \mathrm{h}^{p o l}
$$

where $\mathrm{D}(\mathrm{l})$ denotes the diameter at position 1 along the insulator creepage length [mm] and $\mathrm{h}_{p o l}$ denotes the thickness of the uniform electrolytic pollution layer [mm]. When the insulator surface resistance reaches a critical low value, the critical flashover voltage $\mathrm{V}_{c}$ in $\mathrm{kV}$-peak is given by the formula proposed by Rizk and modified by Holtzhausen [1]:

$$
\mathrm{V}^{c}=\mathrm{k}_{1} \times 10^{-3} \times\left(\frac{R_{c} \times 10^{6}}{L C D}\right) \times L C D
$$

where $\mathbf{R}_{c}$ denotes the critical insulator resistance $\left[\mathrm{M} \Omega\right.$ ], i.e. the same as $\mathrm{R}_{\text {pol }}$, and LCD denotes the total insulator creepage distance [mm]. Constants $\mathrm{k}_{1}$ and $\mathrm{k}_{2}$ are given by

$$
\mathrm{k}_{1}=7.6
$$

and

$$
\mathrm{k}_{2}=0.35 \text {. }
$$

The critical insulator flashover voltage $\mathrm{V}_{C}$ is thus directly proportional to the critical resistance of the insulator $\left(\mathrm{R}_{c}\right)$, which is the same as the value of the surface resistance of the pollution layer $\left(\mathrm{R}_{p o l}\right)[1]$.

Usually, the basic formula given in equation 2.6 is used to explain the effects of creepage distance, volume resistivity and the cross sectional area of an electrolytic pollution layer on the surface resistance of an insulator [1].

$$
\mathrm{R}=\mathrm{R} c=\frac{\rho \cdot L C D}{A}
$$

where $\mathrm{R}$ denotes the surface resistance of the electrolytic pollution layer $[\mathrm{M} \Omega], \rho$ denotes the volume resistivity of the electrolytic pollution layer [M $\Omega . \mathrm{mm}$ ], LCDdenotes the total insulator creepage distance $[\mathrm{mm}]$ and A denotes cross-sectional area of the electrolytic pollution layer $\left[\mathrm{mm}^{2}\right]$. Equation 2.6 shows that the surface layer resistance of an insulator is directly proportional to the total creepage distance. 
Therefore, the power frequency pollution flashover performance of an insulator can be improved by increasing the total creepage distance [1].

\subsubsection{Categories of pollution severity}

Pollution severity can be grouped in four categories, namely light pollution, medium pollution, heavy pollution and very heavy pollution.

Table 2.1 shows the environment that pertains to a specific pollution severity class [23]. However, there are cases when an environment pertains to a different pollution level than the one specified in

Table 2.1. A typical example would be snow and ice in heavy pollution, heavy rain and arid areas [21].

Table 2.1: Relationships between pollution level categories and environment [23]

\begin{tabular}{|c|c|}
\hline Pollution level & Examples of typical environments \\
\hline I - Light & $\begin{array}{l}\text { - Areas without industries and with low density of } \\
\text { houses equipped with heating plants } \\
\text { - Areas with low density of industries or houses } \\
\text { but subjected to frequent winds and/or rainfall } \\
\text { Agricultural areas } \\
\text { Mountainous areas } \\
\text { All these areas shall be situated at least } 10 \mathrm{~km} \text { to } \\
20 \mathrm{~km} \text { from the sea and shall not be exposed to } \\
\text { winds directly from the sea }\end{array}$ \\
\hline II - Medium & $\begin{array}{l}\text { Areas with industries not producing particularly } \\
\text { polluting smoke and/or with average density of } \\
\text { houses equipped with heating plants } \\
\text { Areas with high density of houses and/or } \\
\text { industries but subjected to frequent winds and/or } \\
\text { rainfall } \\
\text { Areas exposed to wind from the sea but not too } \\
\text { close to the coast (at least several kilometers } \\
\text { distant) }{ }^{2}\end{array}$ \\
\hline III - Heavy & $\begin{array}{l}\text { Areas with high density of industries and } \\
\text { suburbs of large cities with high density of } \\
\text { heating plants producing pollution } \\
\text { - Areas close to the sea or in any case exposed } \\
\text { to relatively strong winds from the sea }\end{array}$ \\
\hline I V - Very heavy & $\begin{array}{l}\text { Areas generally of moderate extent, subjected to } \\
\text { conductive dusts and to industrial smoke } \\
\text { producing particularly thick conductive deposits } \\
\text { Areas generally of moderate extent, very close to } \\
\text { the coast and exposed to sea - spray or to very } \\
\text { strong and polluting winds from the sea } \\
\text { Desert areas, characterized by no rain for long } \\
\text { periods, exposed to strong winds carrying sandand } \\
\text { salt, and subjected to regular condensation }\end{array}$ \\
\hline
\end{tabular}


Table 2.2 summarises the Specific Creepage Distances recommended for different pollution severity classes.

Table 2.2: Recommended Specific Creepage Distance for various pollution severity classes for AC and DC voltages [1]

\begin{tabular}{|c|c|c|c|c|}
\hline \multirow{4}{*}{\multicolumn{2}{|c|}{$\begin{array}{r}\text { Pollution } \\
\text { Severity Class }\end{array}$}} & \multicolumn{3}{|c|}{ Minimum Specific Creepage Distance $(\mathrm{mm} / \mathrm{kV})$} \\
\hline & & \multicolumn{2}{|l|}{ AC } & \multirow[t]{3}{*}{ DC } \\
\hline & & \multicolumn{2}{|c|}{ Calculated using system highest voltage of } & \\
\hline & & $\begin{array}{l}U_{m} \\
\text { (Phase to Phase) }\end{array}$ & $\begin{array}{l}U_{m} / \sqrt{3} \\
\text { (Phase to ground) }\end{array}$ & \\
\hline I & Light & 16 & 28 & 20 \\
\hline II & Medium & 20 & 35 & 24 \\
\hline III & Heavy & 25 & 43 & 31 \\
\hline IV & Very Heavy & 31 & 54 & 38 \\
\hline
\end{tabular}

\subsubsection{Critical wetting}

The conductivity of the pollution surface layer is one of the factors that lead to a pollution flashover. Critical wetting occurs when the environment is exposed to the highest surface conductivity [26]. A wetting rate is considered critical whenever the pollution layer is wetted completely such that a pollution flashover is likely to occur. Furthermore, the wetting rate is also considered critical when the pollution layer is not completely wiped away from the insulator surface. A pollution flashover will most likely occur under critical wetting [26]. The critical wetting for the various pollution types can be described as follows [26]:

- Active pollution [26]:

- Conductive pollution: Wetting is always critical.

○ High solubility salts: Wetting is critical for a low wetting rate, for example mist, dew or light rain.

- Low solubility salts: The wetting is critical under high wetting rate such as heavy rain.

- $\quad$ Inert pollution [26]:

○ Hydrophilic pollution: Enhances pollution wetting. 
○ Hydrophobic pollution: Inhibits pollution wetting.

\subsubsection{Pollution site severity assessment methodologies}

\subsubsection{Overview}

Site severity assessment is the measurement of pollution levels and the study of the different weather conditions needed to determine the correct environment for the insulator. The pollution level of a certain area is obtained by measuring the surface deposit on an insulator or by using directional dust deposit gauges [1]. The Insulator Pollution Monitoring Device (IPMD) is used as an alternative pollution severity measuring technique to measure both pre-deposited and instantaneous pollution [1].

Pre-deposited pollution is when the surface conductance is related to the degree of wetting on the insulator surface. This is a natural process. Instantaneous pollution occurs when a highly conductive fog moves into the area causing flashovers, but leaves a very low pollution level on the insulator [7].

The surface conductance of a naturally polluted insulator is obtained using the Insulator Pollution Monitoring Relay (IPMR) so that the pollution severity class can be determined. The IPMR calibration curve is used to convert the measured conductance to an equivalent ESDD value. Both the artificial and natural pollution tests, however, have to be obtained for the calibration curve [7]. The IPMR is used to determine the pollution severity class, help determine the maintenance interval for the insulator and trigger an alarm when measured values exceed maximum permissible values [7].

\subsubsection{Surface pollution deposit technique}

The surface pollution deposit technique shows the natural pollution deposit on insulator for a certain period of time during which natural pollution had already taken place [1]. When the surface pollution deposit technique is not used, the surface pollution deposit measurement on existing insulators can be made as an alternative. However, this measurement is one snapshot at a time, and could thus represent a lower pollution severity for the site than the actual value [1]. The following measurement techniques apply:

- $\quad$ Active pollution - Equivalent Salt Deposit Density (ESDD): 
The ESDD value is defined as the equivalent amount of $\mathrm{NaCl}$ deposit $\left[\frac{\mathrm{mg}}{\mathrm{cm}^{2}}\right]$ required on the surface area of an insulator to yield an electrical conductivity equal to that of the actual deposit dissolved in the same amount of water. The ESDD technique involves the process of washing the pollution layer with distilled water and measuring the conductivity of the solution obtained [1]. The ESDD value thus determines the severity of the contamination present on the insulator surface [21].

- $\quad$ Inert pollution - Non-Soluble Deposit Density (NSDD):

The NSDD shows the amount of non-soluble pollution deposits per square centimetre of the insulator surface. The NSDD measurement is obtained from the mixed solution of the ESDD measurement. This liquid is then filtered through a pre-dried, clean and weighed filter paper. The contaminated filter paper is then dried and weighed [1].

\subsubsection{Directional dust deposit gauge technique}

The Directional Dust Deposit Gauge (DDDG) has four vertical tubes with a slot on the side. These slots are arranged such that they face north, south, east, and west. There is a removable container at the bottom of each slot which collects the deposits blown into the slots, and the containers are removed on a monthly basis for testing. The contents are mixed with $500 \mathrm{ml}$ of demineralised water and the conductivities of the mixed solutions are measured. The pollution index is defined as the average of the four conductivities obtained from the four directions in $\left[\frac{\mu S}{\mathrm{~cm}}\right]$ and normalised to a 30 - day interval [1]. Figure 2.6 show some design details of the DDDG. 

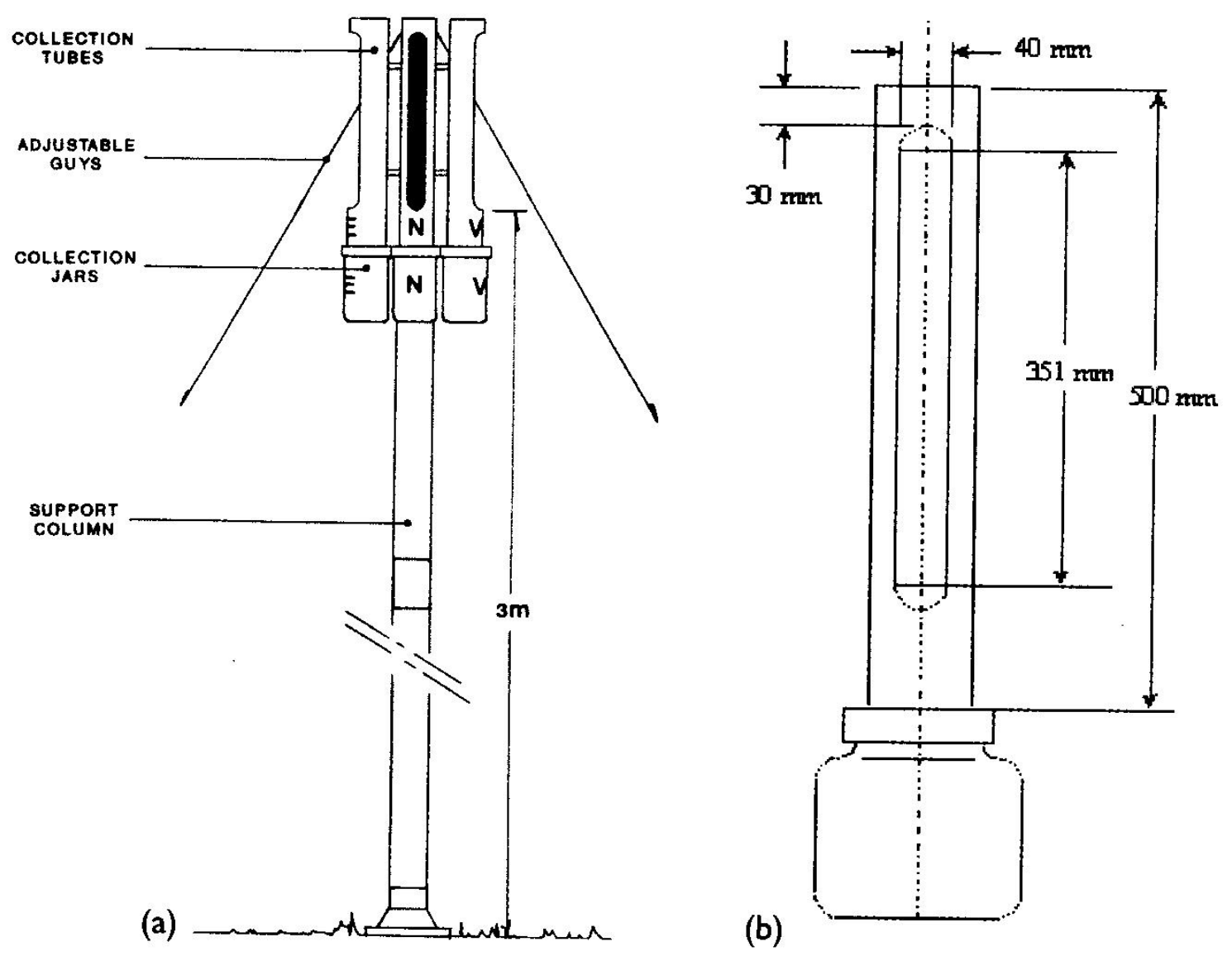

Figure 2.6: DDDG design details: (a) As installed, (b) Dimensions

\subsubsection{Site severity class}

The site severity class can be obtained from the surface deposit and dust gauge measurements [1]. This involves the following:

- $\quad$ Surface deposit index:

The surface index is given by the ESDD value. The relationship between the respective pollution severity classes and the surface deposit index over a period of a year is given by Table 2.4. A measured NSDD value above $2\left[\frac{\mathrm{mg}}{\mathrm{cm}^{2}}\right]$ requires an increase by one level for the site severity class. 
Table 2.3: The relationship between surface deposit index and severity class

\begin{tabular}{|c|l|l|}
\hline $\begin{array}{c}\text { Surface deposit index, ESDD [mg/cm²] } \\
\text { (monthly maximum) }\end{array}$ & $\begin{array}{l}\text { Pollution } \\
\text { Severity Class }\end{array}$ \\
\hline$<0.06$ & I & Light \\
\hline $0.06-0.12$ & II & Medium \\
\hline$>0.12-0.24$ & I & Heavy \\
\hline$>0.24$ & IV & Very Heavy \\
\hline
\end{tabular}

- Dust deposit gauge pollution index:

The site severity class can be obtained from the monthly average or the maximum of the pollution indices measured by the dust gauge. The relationship between the site severity class and the pollution index, over one year, is given by Table 2.5. The site severity class should be increased by one level if a high NSDD is expected such as encountered in the vicinity of a cement factory [1].

Table 2.4: The relationship between DDG pollution index and severity class [1]

\begin{tabular}{|c|c|c|c|}
\hline \multicolumn{2}{|c|}{ Dust deposit gauge pollution index, PI[ S/am] } & \multicolumn{2}{c|}{ Pollution } \\
\hline (monthly average) & (monthly maximum) & \multicolumn{2}{c|}{ severity class } \\
\hline 0 to 75 & 0 to 175 & I & Light \\
\hline 76 to 200 & 176 to 500 & II & Medium \\
\hline 201 to 350 & 501 to 850 & II & Heavy \\
\hline$>350$ & $>850$ & Very Heavy \\
\hline
\end{tabular}

\subsubsection{Natural pollution test stations}

In 1995, Vosloo established the Brandsebaai insulator pollution test station north of Koeberg along the West Coast of South Africa. Different insulator materials and creepage distances were tested. The results showed that SR performed better compared to EPDM, Enhanced Silicone Polymer (ESP) and ceramic units. The results obtained at the Brandsebaai pollution test station were similar to the results obtained at Elands Bay and the Koeberg insulator test station [3].

In 1996 Vosloo, together with Hartings and Gutman, developed the Kelso on-line insulator test station and the Clansthal insulator test tower along the East Coast of Natal. This research focused on the testing of insulators with different profiles, different materials, specific creepage distances, arcing distances and manufacturer. The results obtained from this experiment were similar to the results obtained from Koeberg [3]. 
In 1997, another insulator test tower was developed by Vosloo in Alexanderbaai. However, during this set of experiments, it was observed that the SR insulators performed much better than the glass insulators. Furthermore, there was also a difference between the two SR units when compared to one another [3].

Research conducted on insulators along the west coast of South Africa has indicated that SR insulators perform much better than porcelain, glass, EPDM and cycloaliphatic units, which have shown poor performance in terms of material and leakage current. The silicone grease and coatings have showed better performance when compared to the bare porcelain insulators. There resistive glazed porcelain also yielded good results [3].

The collection of data at the ESKOM Koeberg test site was discontinued for a while. This was because of the equipment failing as a result of harsh environmental conditions. This led to the development of the Kuils River and Elands Bay Pollution test sites. These sites were chosen because of the severe pollution experienced in those areas [2]. Insulators made of different materials and with different specific creepage distances were installed at both test sites. Some ceramic insulators at the Elands Bay test site had to be greased or had to have their creepage distance extended after flashovers. The follow-up experiments used mainly non-ceramic insulators [2].

The Kuils River test site showed no flashover or excessive leakage current. There, the pollution appeared to be in the very light range. After a year the test was stopped and the test was moved to the original Koeberg test site [2].

The results from the pollution measurements at the Elands Bay test site after two years can be summarized as follows $[2,3]$ :

- Insulators with a minimum SCD of $30 \mathrm{~mm} / \mathrm{kV}$ should be used for that area.

- Cyclo-aliphatic insulators are not suitable for use in a severely polluted area, since they showed irreversible surface degradation.

- $\quad \mathrm{SR}$ and EPDM insulators with Specific Creepage Distance of $25 \mathrm{~mm} / \mathrm{kV}$ or more should be used for this area (severely polluted area), since these gave acceptable results. 


\section{Design of the Tracking Wheel Tester}

\subsection{Overview}

This chapter gives details of the design and operation of the Tracking Wheel Tester (TWT) facility, including the mechanical design, the design of the HVAC and HVDC excitation circuits and the associated control and measuring instrumentation. The TWT represents a test system to evaluate the resistance of insulators to tracking and erosion and can be used to compare the performance of different materials [27].

Figure 3.1 shows a flow diagram of the operation of the TWT. The rotating wheel rotates at a constant speed of three revolutions per minute. The test specimen is dipped into the salt water solution for $60^{\circ}$ of a revolution. The test specimen is then withdrawn from the salt water solution (resting phase) and the salt water solution is allowed to drip from the surface of the test specimen. This process occupies another $60^{\circ}$ of a revolution. The test voltage is then applied to the insulator for an interval of $180^{\circ}$ of one revolution (excitation phase). Finally, the test specimen goes through a final resting phase for $60^{\circ}$ of one revolution, before the cycle is repeated. 


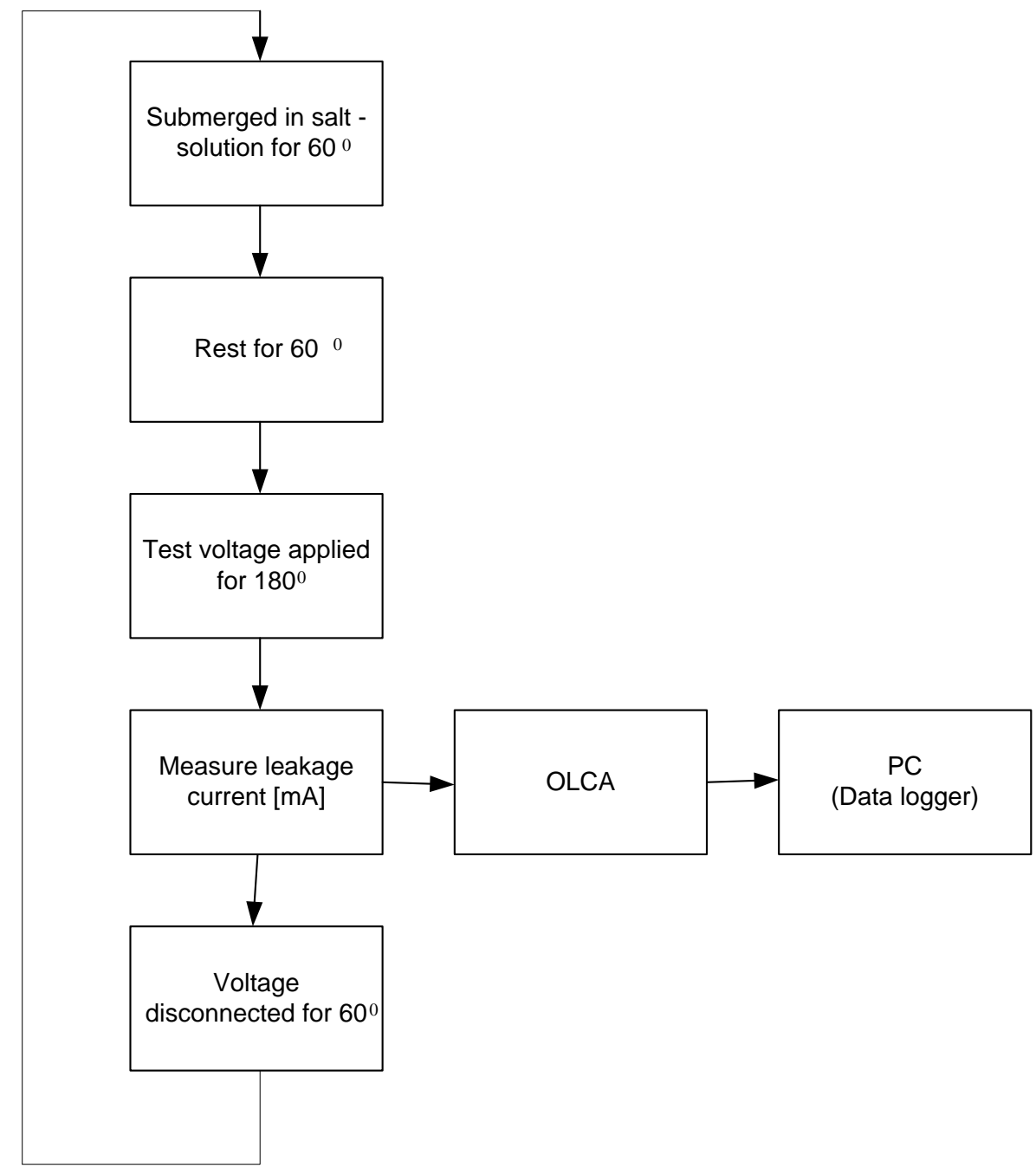

Figure 3.1: Operating sequence of the Tracking Wheel Tester

\subsection{Design of the Tracking Wheel Tester}

\subsubsection{Mechanical structure}

The mechanical structure of the TWT was designed according to the IEC 61302 specification. Figure 3.2 shows the tracking wheel tester as built for the first series of tests conducted with HVAC excitation. During this test, the performance of the TWT was evaluated for a period 1084 hours and 50 minutes. 


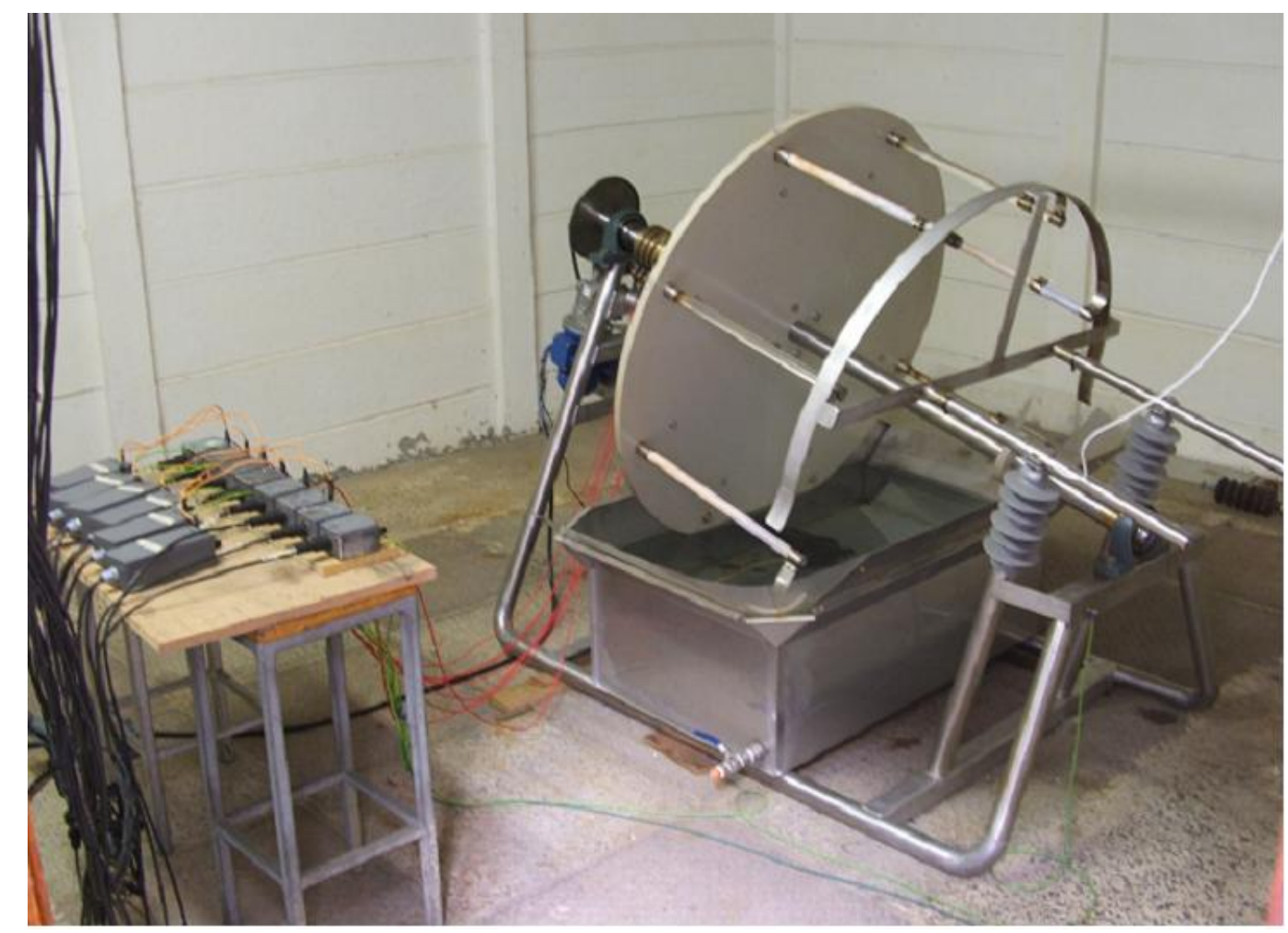

Figure 3.2: The Tracking Wheel Tester (TWT)

\subsubsection{Design of the electrical systems}

\subsubsection{Overview}

The electrical and electronic systems associated with the TWT can be summarized as follows [5]:

- Electrical drive system:

The drive system consists of a worm geared motor with a chain drive for the rotating wheel and the associated control logic to ensure save operation of the system.

- Protection system:

The protection system detects flashovers and stops the operation.

- Excitation system:

The excitation system generates HV excitation

- Measuring system:

The measuring system measures and records the leakage current for the individual test samples. 
- $\quad$ Single phase supply transformer.

- Control circuit.

- $\quad$ Protection circuit.

- $\quad$ OLCA (Online leakage current analyzer).

Figure 3.3 shows an electrical schematic of the TWT.

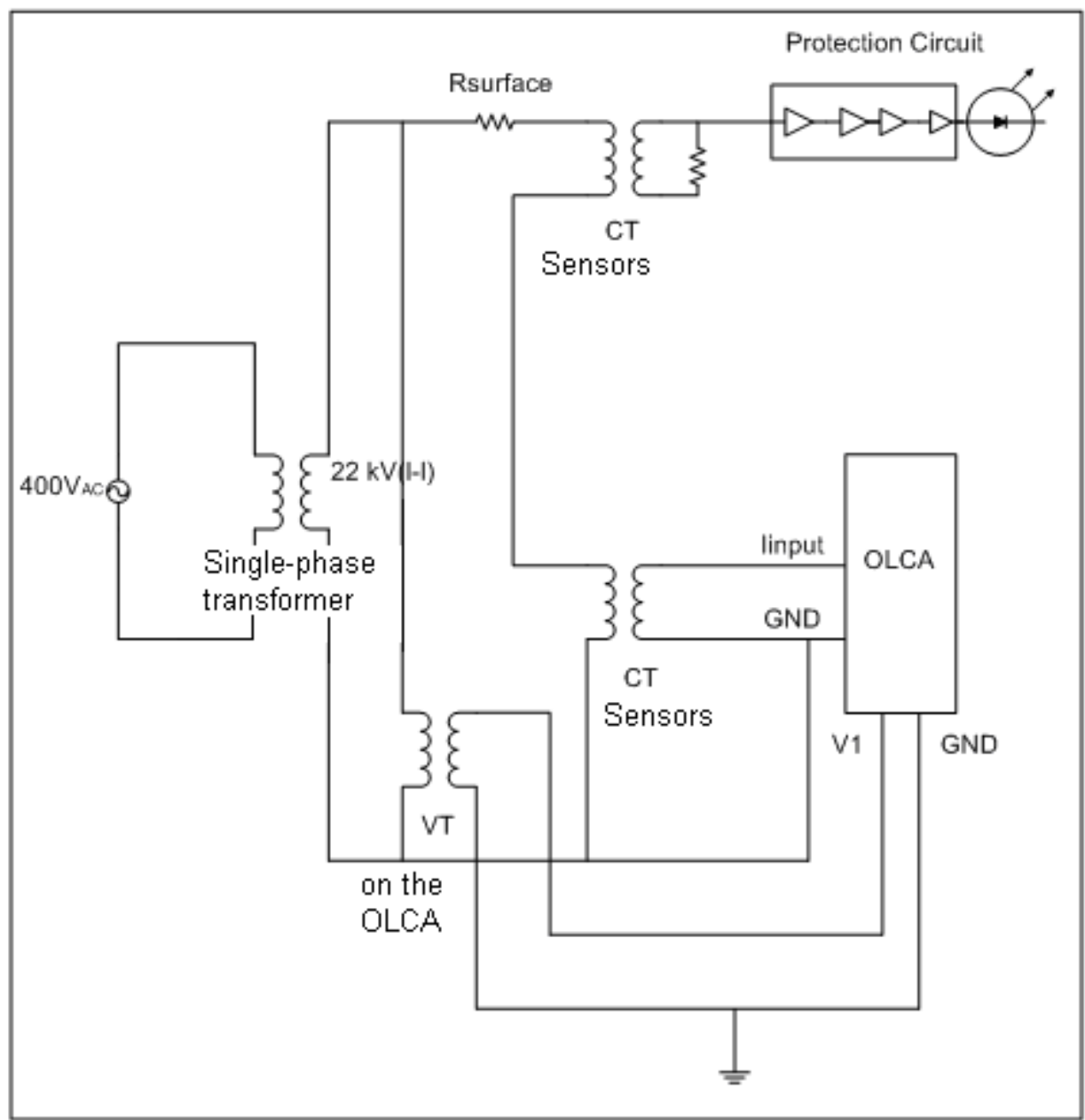

Figure 3.3: Schematic diagram of the Tracking Wheel Tester for the AC system

\subsubsection{Control circuit}

The control circuit is necessary for effective operation when dealing with a High Voltage (HV) area. The combination of the emergency stop button reset, door of the chamber closed and the trip circuit set, and start button being pressed will initiate the test sequence. Therefore rotation of the wheel will commence and eventually the excitation voltage will be applied [5]. 


\subsubsection{Worm geared drive motor}

A worm geared motor rotates the wheel via a chain drive at a constant speed of three revolutions per minute. The motor supply is separated from the transformer and control circuit. The voltage source has a tripping device which disconnects the test voltage when a failure occurs. This is done without stopping the rotation of the wheel $[5,27]$.

\subsubsection{Excitation system}

Two excitation sources were used in during the project, namely and High Voltage Alternating Current (HVAC) source and a High Voltage Direct Current (HVDC) source. These can be summarized as follows:

- $\quad$ High Voltage Alternating Current (HVAC) excitation source

The HVAC excitation source relies on a three-phase 22000:400 Delta-Star transformer that is reverse energized to generate the HVAC excitation voltage as shown in Figure 3.3. Only the centre limb of the transformer is utilized to function as a single phase $230 \mathrm{~V}: 22 \mathrm{kV}$ step-up transformer. The input voltage to the step-up transformer is obtained from the $230 \mathrm{~V}, 50 \mathrm{~Hz}$ mains supply and is regulated by means of variable autotransformer to control the amplitude of the HV excitation voltage.

- $\quad$ High Voltage Direct Current (HVDC) excitation source

The basic HVDC source consists of a three-phase transformer, a rectifier unit, a capacitor bank and a resistive voltage divider to measure the output voltage. Figure 3.4 shows the circuit topology of the HVDC source.

The high voltage supply was obtained from a $400 \mathrm{~V} / 22 \mathrm{kV}$ three phase step-up transformer (the same as used in the HVAC circuit) used with a star connection on the low voltage side and a delta connection on its high voltage side. The three phase transformer had a Dyn11 (cbayn) vector group with the electrical specifications given in Appendix D. The step-up transformer is supplied via variable autotransformer to control the output voltage. Due to the lack of a starpoint on the HV side, one of the phase terminals were connected to ground to provide a return path for the remaining two phases, each of which was connected to the load via a diode rectifier. Output filtering is provided by 
smoothing capacitors. Fuses were used to protect the system. Table 3.1 summarizes the output specifications used in the design of the HVDC source.

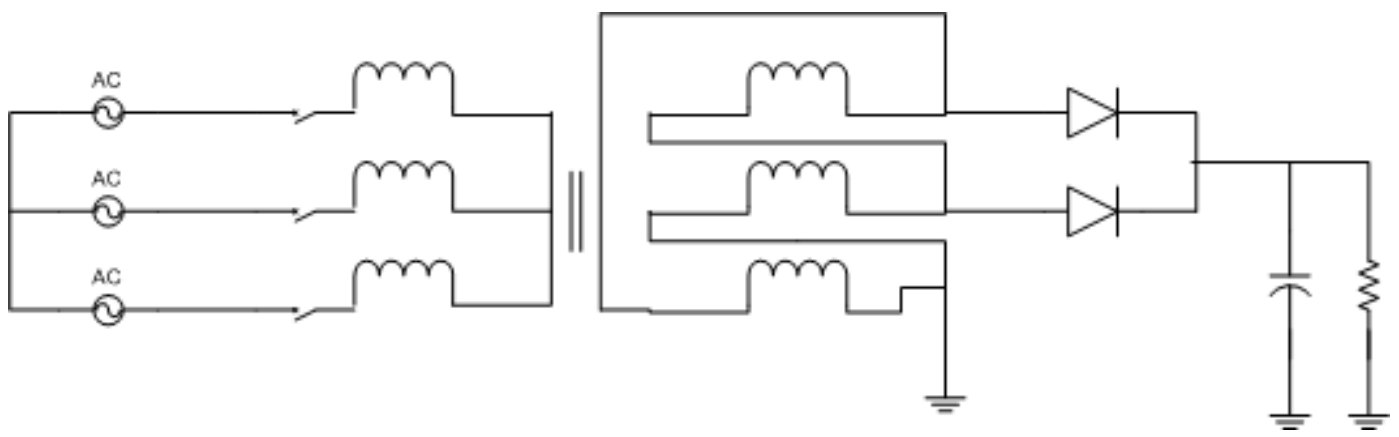

Figure 3.4: Circuit topology of the HVDC Source

Table 3.1: Rectifier specifications

\begin{tabular}{|l|l|}
\hline Rated Output Voltage & $13.6 \mathrm{kV}_{D C(L-N)}$ \\
\hline Rated Current & $1 \mathrm{~A}$ \\
\hline Rated ripple factor (@ 1A) & $3 \%$ \\
\hline
\end{tabular}

Figure 3.5 shows the HVDC source as used in the investigation, including the three-phase step-up transformer, rectifier module and capacitor bank. Figure 3.6 shows the rectifier module while Figure 3.7 shows the capacitor bank.

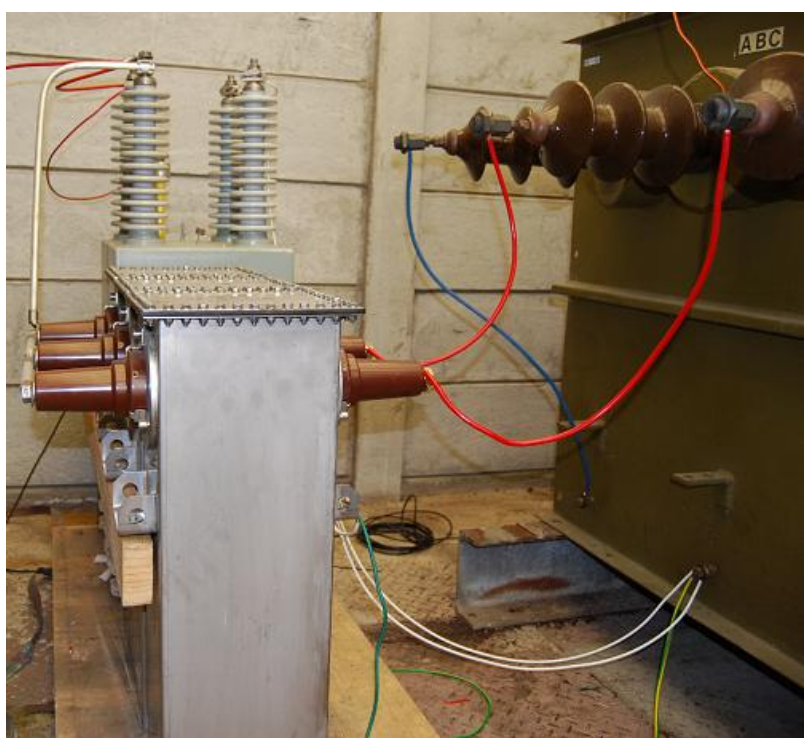

Figure 3.5: The HVDC source with the rectifier and capacitor, as well as part of the transformer 


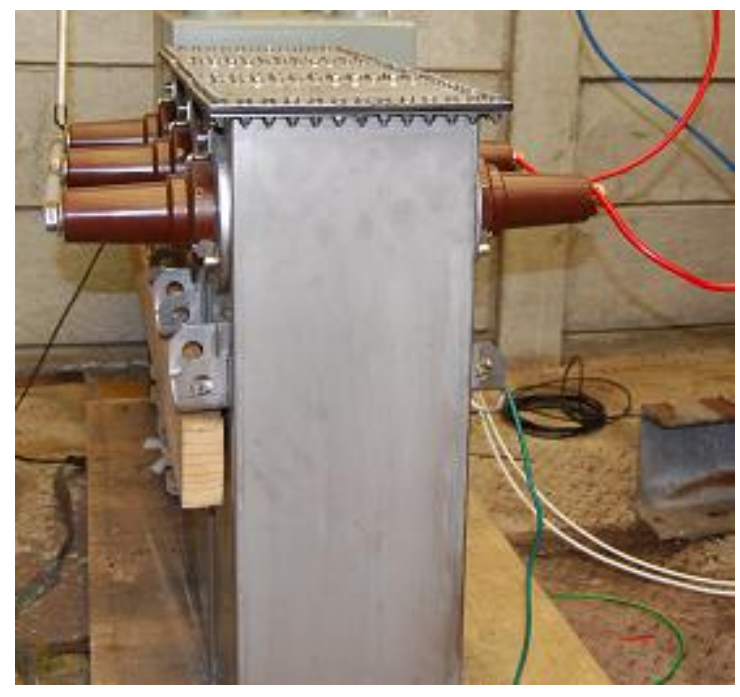

Figure 3.6: The rectifier module

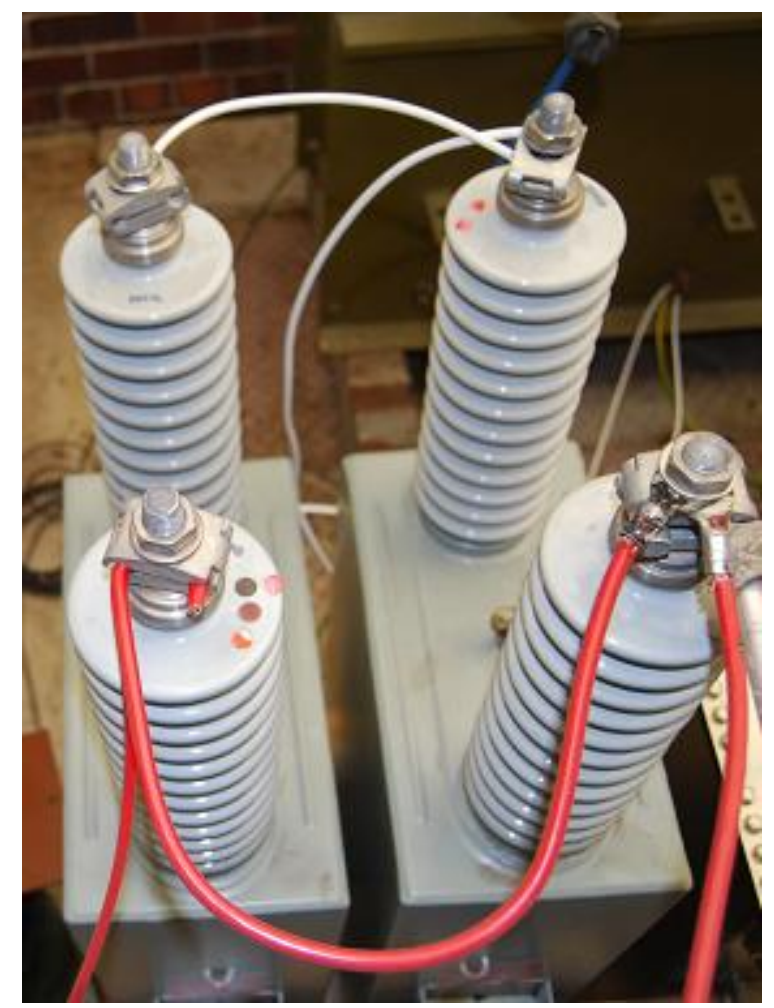

Figure 3.7: The smoothing capacitor bank

\subsubsection{Measuring instrumentation}

A commercial Online Leakage current Analyser (OLCA), using dedicated current sensors, was used to measure the peak leakage currents for the six test insulators. A separate set of current sensors were used to supply the protection circuit. Figure 3.8 and Figure 3.9 show photographs of the OLCA current sensors and OLCA measuring instrument respectively. 


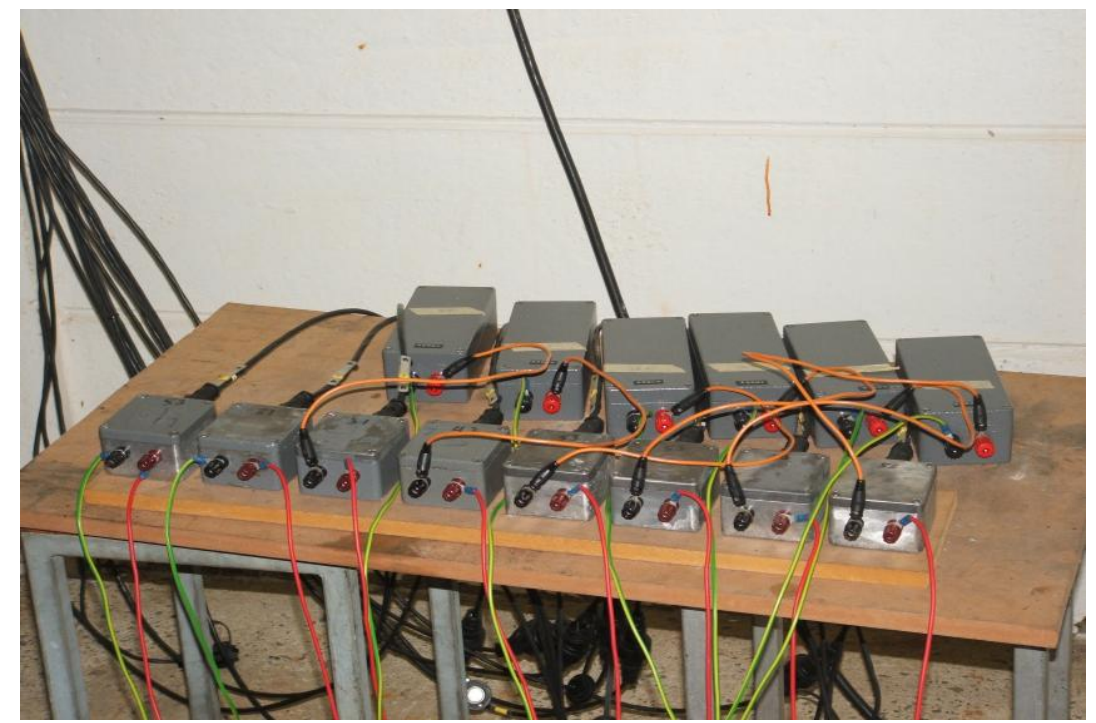

Figure 3.8: The OLCA current sensors

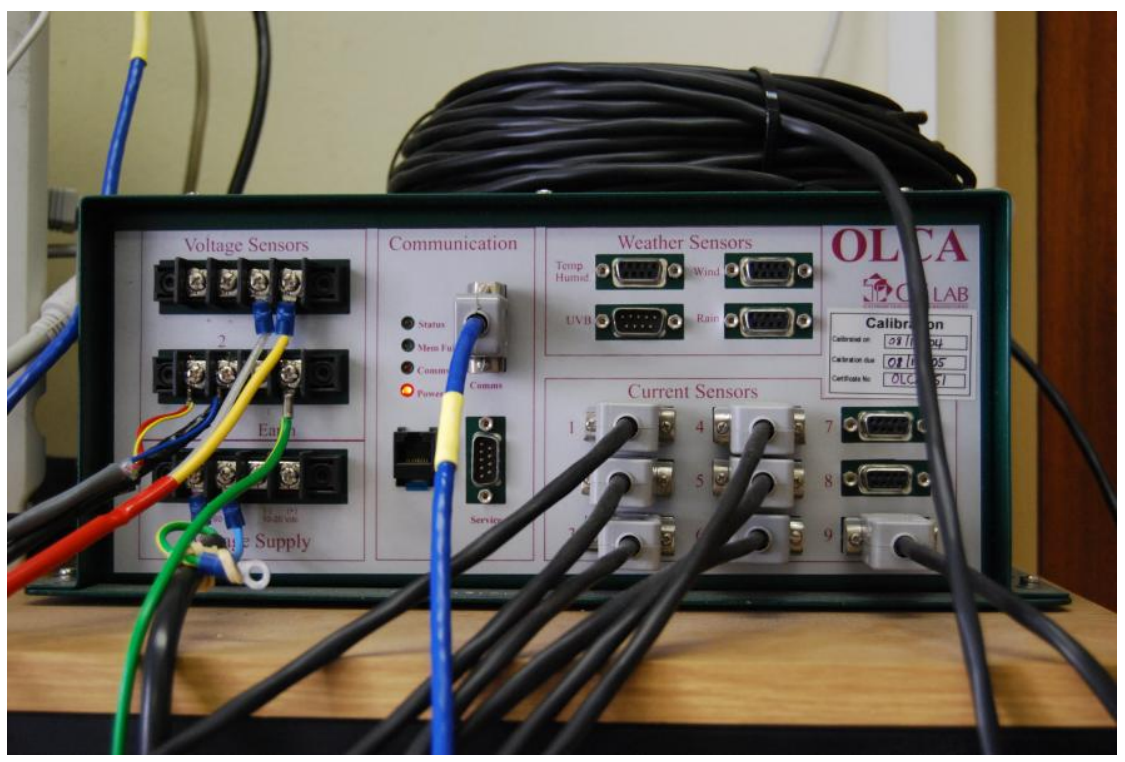

Figure 3.9: The OLCA measuring instrument

\subsubsection{The protection circuit}

The protection circuit monitors the leakage currents for the individual test samples and performs the following functions:

- Detects an overcurrent condition such as caused by flashover of one of the test samples (sample failure).

- Disconnects the HV excitation supply in case of an overcurrent condition.

- Indicates by means of a Light Emitting Diode (LED) which test sample initiated the overcurrent trip condition. 
Sample failure, as defined according to the IEC 61302 standard, occurs if one of the following takes place [27]:

- A flashover

- $\quad$ The r.m.s. leakage current of the test sample exceeds $300 \mathrm{~mA}$

Figure 3.10 shows a circuit diagram for one channel of the multi-channel protection system while Figure 3.11 shows a functional block diagram for the protection circuit. The circuit consists of the following sub-circuits:

- $\quad$ Non-inverting buffer/amplifier.

- $\quad$ A $2^{\text {nd }}$ order Sallen-Key low pass filter.

- A window comparator.

- A latching circuit.

- $\quad$ Relay driver (to trip supply).

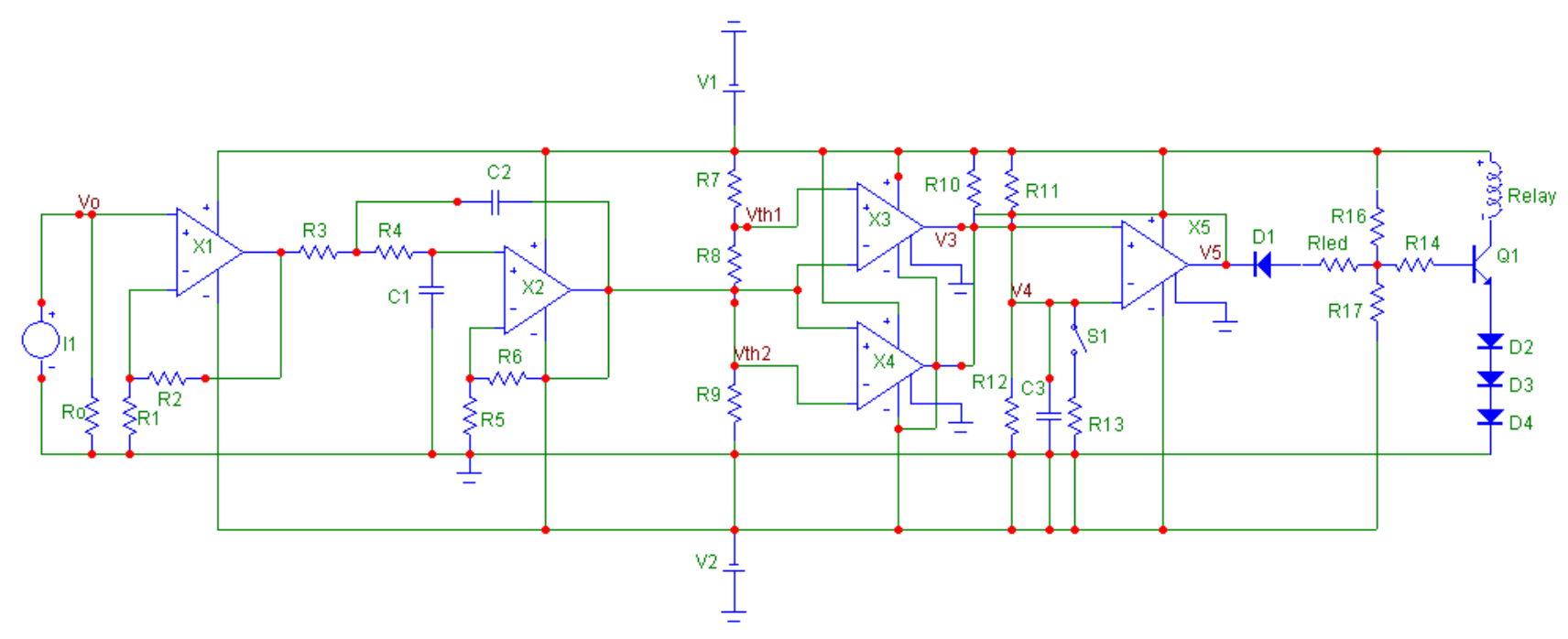

Figure 3.10: Circuit diagram of the protection circuit. 


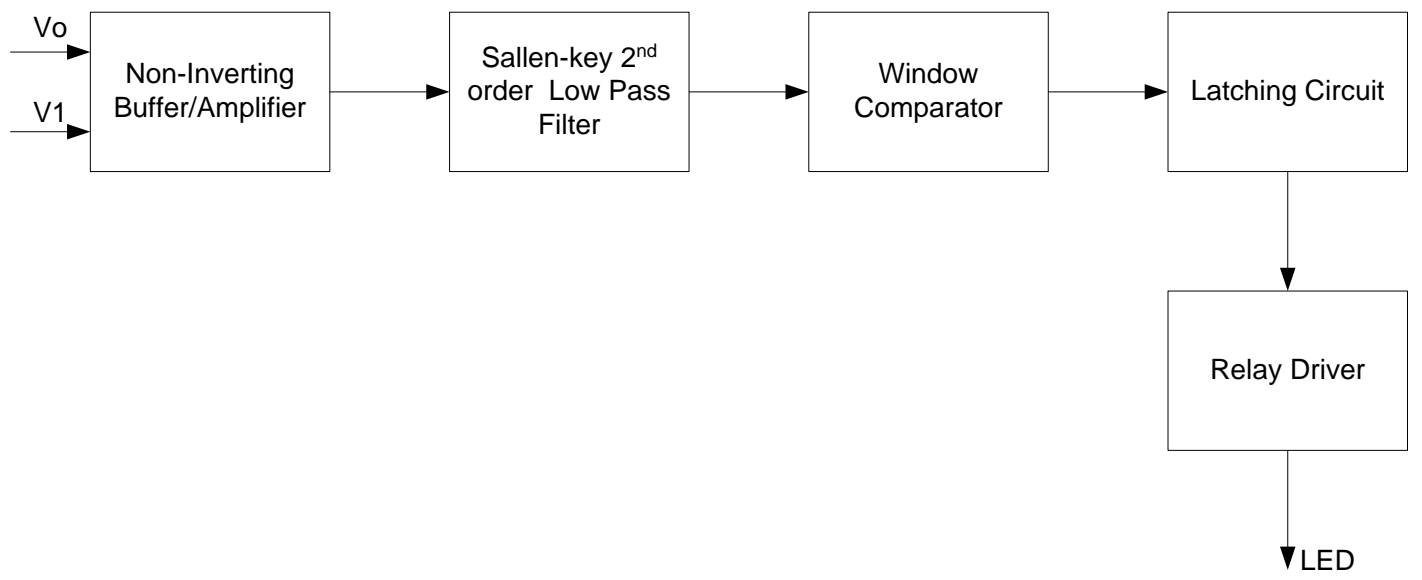

Figure 3.11: Block diagram for the protection circuit.

The operation of the circuit can be described as follows:

- $\quad$ The current input signal from the current sensor is converted to a voltage signal $\mathrm{V}_{0}$ by the input resistor and buffered by the input buffer.

- The output from the non-inverting buffer amplifier is filtered by a Sallen-Key $2^{\text {nd }}$ order low pass filter to remove high frequency noise.

- $\quad$ The window comparator compared the output signal $\left(\mathrm{V}_{2}\right)$ from the filter to the positive and negative threshold voltages $\mathrm{V}_{t h 1}$ and $\mathrm{V}_{t h 2}$. The output signal from the window comparator will be either high $(+15 \mathrm{~V})$ or a low $(0 \mathrm{~V})$, depending on whether the input voltage exceeds the boundaries defined by the threshold voltages. Figure 3.12 shows the voltage waveforms to illustrate the operation of the window comparator, with $\mathrm{V}_{t h 1}$ and $\mathrm{V}_{t h 2}$ set to $10 \mathrm{~V}$ and $-10 \mathrm{~V}$ respectively.

- The output of the window comparator $\left(\mathrm{V}_{3}\right)$ drives the latching circuit. When failure occurs, the output of the window comparator $\mathrm{V}_{3}$ will be OV. This condition will latch the output of the latching circuit $\mathrm{V}_{5}$ in the low state, thereby activating the LED represented by D1.

During normal operation reset switch S1 will be open. If closed, it resets the circuit and the latching circuit will be cleared. Therefore, the output voltage $\left(\mathrm{V}_{5}\right)$ will be high, since $\mathrm{R}_{13}$ in parallel with $\mathrm{R}_{12}$ will pull $\mathrm{V}_{4}$ below $\mathrm{V}_{3}$. 
There is a separate latching circuit and window comparator for each channel, with only one relay driver and reset button common to all the channels. A $15 \mathrm{~V}$ voltage supply was used for the protection circuit. The design uses a 5A SPST relay with a coil resistance of $300 \Omega$ and contact ratings of 5A @ 120V AC with a 2N2222A npn transistor to drive the relay.

During normal operation the transistor is on and the relay is energized, providing failsafe operation in case of current sensor failure or loss of supply. When a failure occurs, the transistor switches off and the relay is de-energized to trip the control circuit. The excitation supply to the test insulators will be disconnected, but the rotating wheel continues to rotate [5].

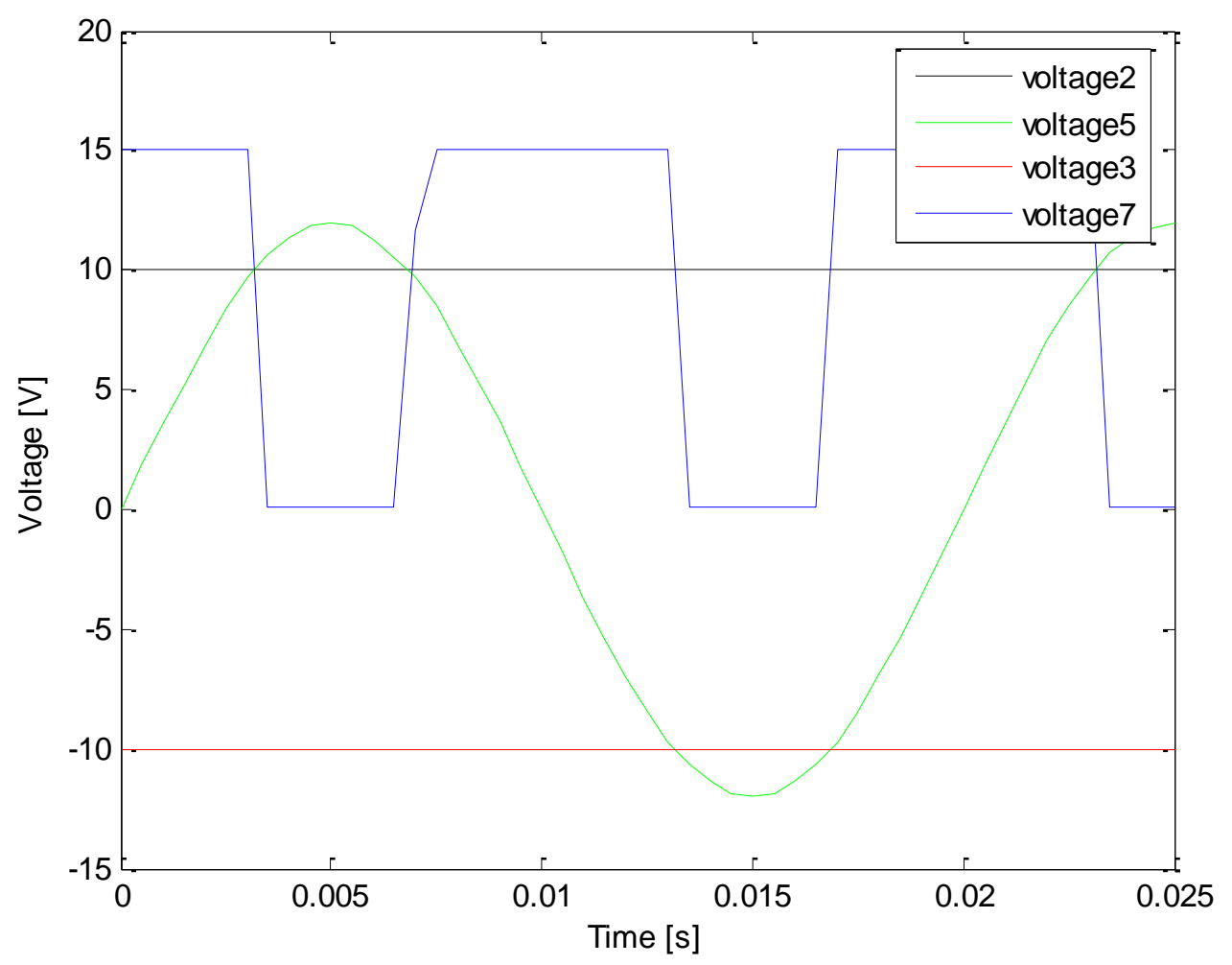

Figure 3.12: Operation of the window comparator 


\section{Summary of Test Procedures and Results}

\subsection{Overview}

This chapter summarizes the results obtained from all the experiments. Two series of test were conducted. These can be summarized as follows:

- $\quad$ First series of tests:

The first test series involved aging tests of RTV SR coated glass insulator rods with HVAC excitation using the procedures specified the IEC 61302 standard. Following completion of the experiments, a number of recommendations were derived with the view to design the second series of tests. Table 4.1 summarizes the samples tested in this series of tests.

- $\quad$ Second series of tests:

The second series of tests involved aging tests of actual insulators of different materials and coatings and Specific Creepage Distance (SCD) with HVAC, positive polarity HVDC and negative polarity HVDC excitation. The test procedures used were derived from the IEC 61302 and the IEC 62217 standards summarized in appendix $\mathrm{C}$.

Table 4.2 and Figure 4.1 summarize the samples tested in this series of tests. Specific Creepage Distance uses the phase to phase system voltage, while the unified Specific Creepage Distance uses the phase to ground system voltage.

Table 4.1: Description of the samples tested in test series 1 .

\begin{tabular}{|l|l|l|l|}
\hline Channel & Material & $\begin{array}{l}\text { Creepage distance } \\
{[\mathbf{m m}]}\end{array}$ & $\begin{array}{l}\text { Specific creepage } \\
\text { distance }[\mathbf{m m} / \mathbf{k V}]\end{array}$ \\
\hline Channel 1 & Glass & 277 & 16 \\
\hline Channel 2 & RTV SR coated glass & 277 & 16 \\
\hline Channel 3 & Glass & 346 & 20 \\
\hline Channel 4 & RTV SR coated glass & 346 & 20 \\
\hline Channel 5 & Glass & 433 & 25 \\
\hline Channel 6 & RTV SR coated glass & 433 & 25 \\
\hline
\end{tabular}


Table 4.2: Description of the samples tested in test series 2.

\begin{tabular}{|l|l|l|l|}
\hline Channel & Material & $\begin{array}{l}\text { Creepage distance } \\
{[\mathbf{m m}]}\end{array}$ & $\begin{array}{l}\text { Unified specific } \\
\text { creepage distance } \\
{[\mathbf{m m} / \mathbf{k V}]}\end{array}$ \\
\hline Channel 1 & EPDM & 380 & 28 \\
\hline Channel 2 & HTV SR & 380 & 28 \\
\hline Channel 3 & HTV SR & 380 & 28 \\
\hline Channel 4 & HTV SR & 476 & 35 \\
\hline Channel 5 & Porcelain & 380 & 28 \\
\hline Channel 6 & RTV SR coated porcelain & 380 & 28 \\
\hline
\end{tabular}
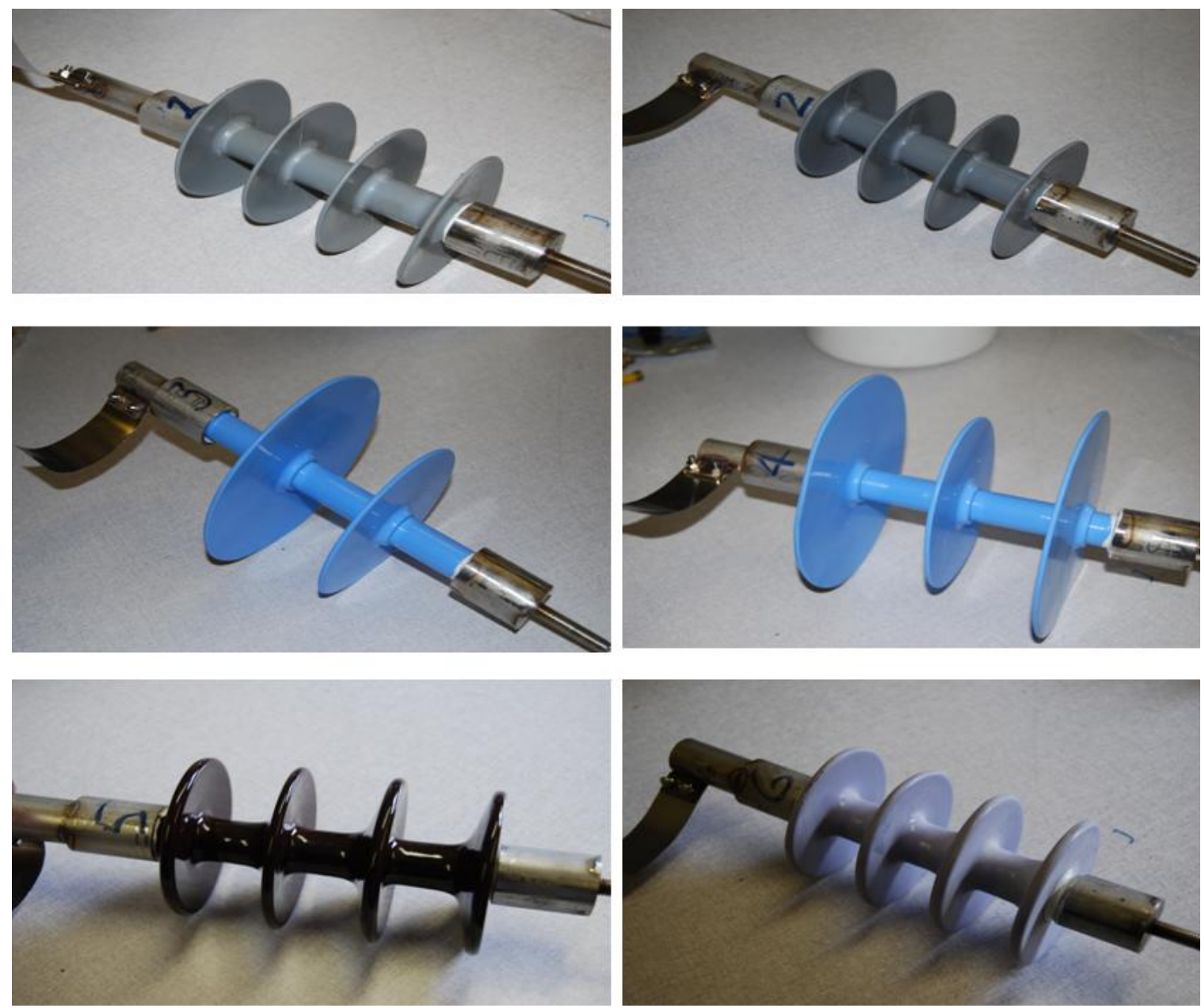

Figure 4.1: The six insulator samples tested during the second series of tests with HVAC, positive polarity HVDC and negative polarity HVDC 


\subsection{First test series: HVAC excitation}

\subsubsection{Overview}

Figure 4.2 shows the TWT with the test samples as used for first series of tests. The tests were conducted according the procedures specified in the IEC 61302 standard. The main objective of the experiment is to compare the aging performance of the different test specimens with different creepage lengths when exposed to a liquid contaminant and HVAC electric stress. Furthermore, this test compares the resistance to tracking and erosion of similar materials [27].

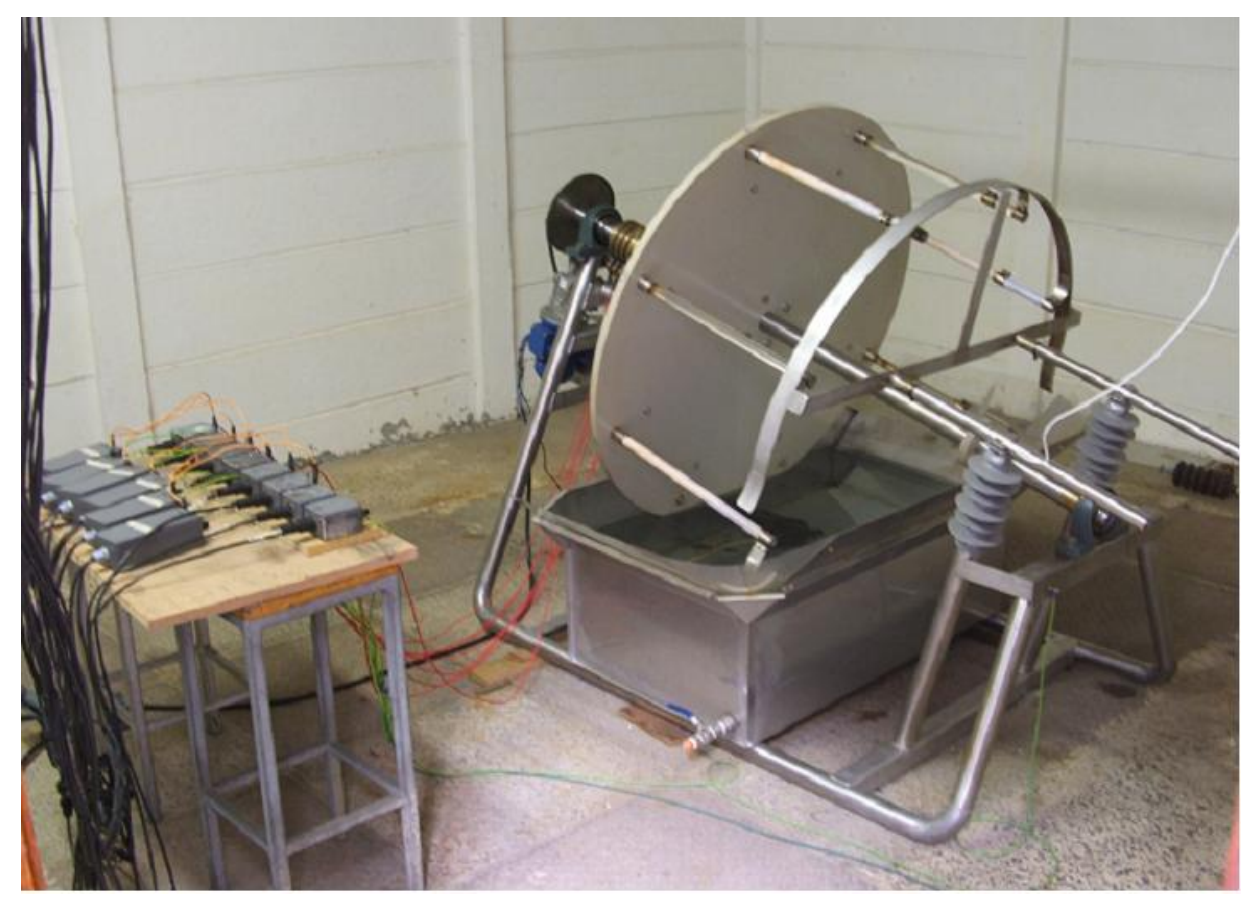

Figure 4.2: The tracking wheel tester with the test samples used in the first series of tests.

\subsubsection{Test methodology and test procedures}

A total of six insulator rods, namely three RTV SR coated glass and three glass samples as summarized in Table 4.1, were tested simultaneously using the TWT. Three creepage distances were used for each of the test materials, namely $277 \mathrm{~mm}$, $346 \mathrm{~mm}$ and $433 \mathrm{~mm}$.

During the test, the insulator rods are dipped into a specified salt-solution and an excitation voltage of $10 \mathrm{kV}$ r.m.s voltage is applied across the insulator. The saltsolution is prepared by adding Sodium Chloride $(\mathrm{NaCl})$ to de-ionized or distilled water to achieve a conductivity of $1.33[\mathrm{mS} / \mathrm{cm}] \pm 5 \%$ at $25^{\circ} \mathrm{C}$ at the beginning of the test. The temperature of the salt solution should be maintained at $25 \pm 5^{\circ} \mathrm{C}$. 
The following procedure was used at the beginning of the experiment and for restarting the experiment after an interruption:

- $\quad$ Switch on the TWT drive motor.

- Lock the door to the test chamber and remove the earth stick attached to the transformer.

- $\quad$ Reset the emergency stop button.

- $\quad$ Switch on the main circuit breaker (400 A).

- $\quad$ Set the variac to zero.

- $\quad$ Switch on the transformer supply circuit breaker (100 A).

- $\quad$ Reset the protection circuit.

- Switch on the control circuit supply.

- $\quad$ Adjust the variac until a voltage of $10 \mathrm{kV}$ is displayed on the $\mathrm{HV}$ side of the transformer.

In order to make sure that repeatable results are obtained and that the tests conform to the relevant test standard, the test arrangement required close supervision. The following operating procedures were implemented to this effect:

- Continuous monitoring of the water level with the view to maintain the water level to within $\pm 10 \mathrm{~mm}$.

- Continuous monitoring of the conductivity with the view to maintain the conductivity at $1.33 \pm 5 \%\left[\frac{\mathrm{mS}}{\mathrm{cm}}\right]$ at $25^{\circ} \mathrm{C}$. The conductivity referred to $20{ }^{\circ} \mathrm{C}$ is $1.19 \pm 5 \%\left[\frac{\mathrm{mS}}{\mathrm{cm}}\right]$.

- Continuous monitoring of the temperature of the liquid with the view to maintain the temperature at $(25 \pm 5){ }^{\circ} \mathrm{C}$.

- Examine the wheel every 24 hours to make sure that hardware is working properly.

The following operating procedure was followed in case of a fault: 
- The emergency stop button is pressed so that the LV supply can be disconnected.

- $\quad$ The motor is switched off.

- $\quad$ Open the chamber door and apply the earth stick to the HV tap and remove the failed specimen.

- $\quad$ Replace with new specimen and restart the process.

The following operating procedure was followed to stop the machine:

- $\quad$ Set the variac to zero.

- Switch off the control circuit supply.

- $\quad$ Switch off the transformer supply circuit breaker (100 A).

- $\quad$ Unlock the door and apply the earth stick to the HV Supply.

\subsubsection{Summary of test results}

\subsubsection{Leakage current}

A digital storage oscilloscope was used to record the typical current waveforms of one of the insulator specimens over one complete cycle. The graph in Figure 4.3 shows the initial current waveform from the instant that the voltage is applied.

Figure 4.4 illustrates the irregularities in the current waveform as a result of the dry band formation, i.e. after the voltage has been applied for some time. This distortion occurs mostly in the regions of the waveform peaks. The change in the peak current amplitudes between the results shown in Figure 4.3and Figure 4.4 is due the loss of the liquid contaminant on the surface of test specimen as the sample rotates with the excitation voltage applied. 


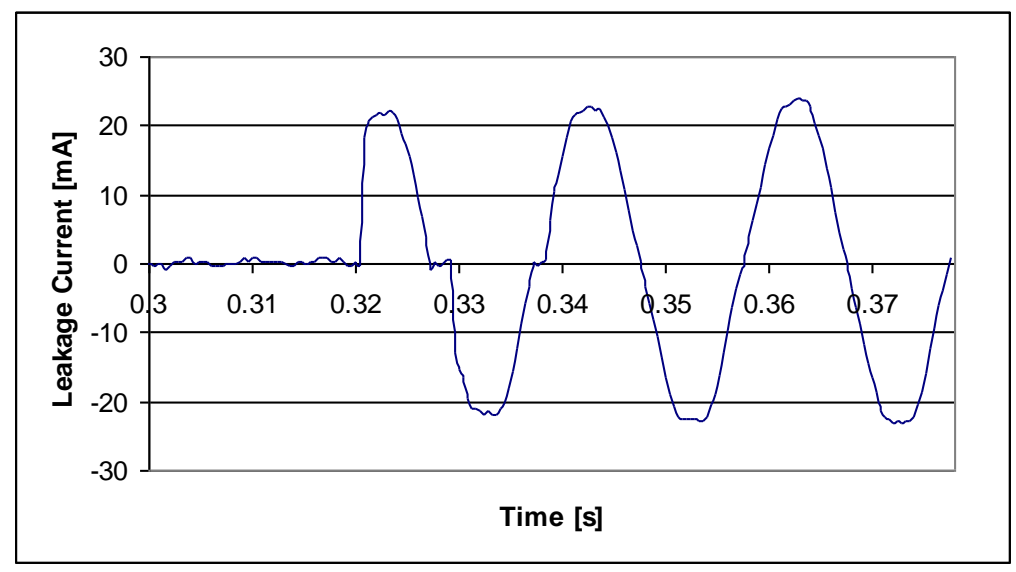

Figure 4.3: Leakage current $[\mathrm{mA}]$ waveform before and after the voltage is applied

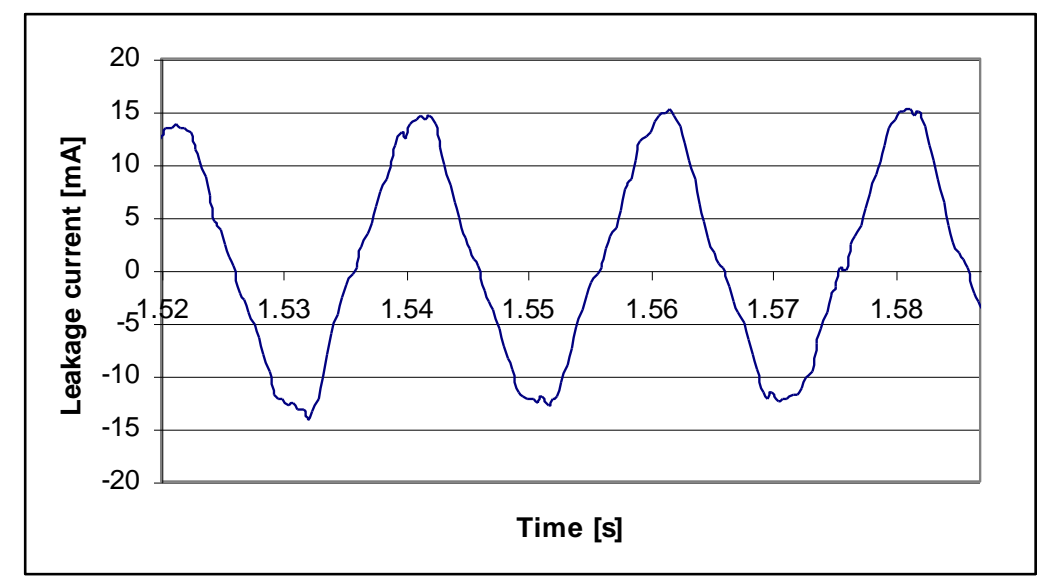

Figure 4.4: Leakage current $[\mathrm{mA}]$ waveform during dry band formation

Figure 4.5 shows the current waveform recorded towards the end of the excitation phase. At this point the waveform tends to become distorted and discontinuous.

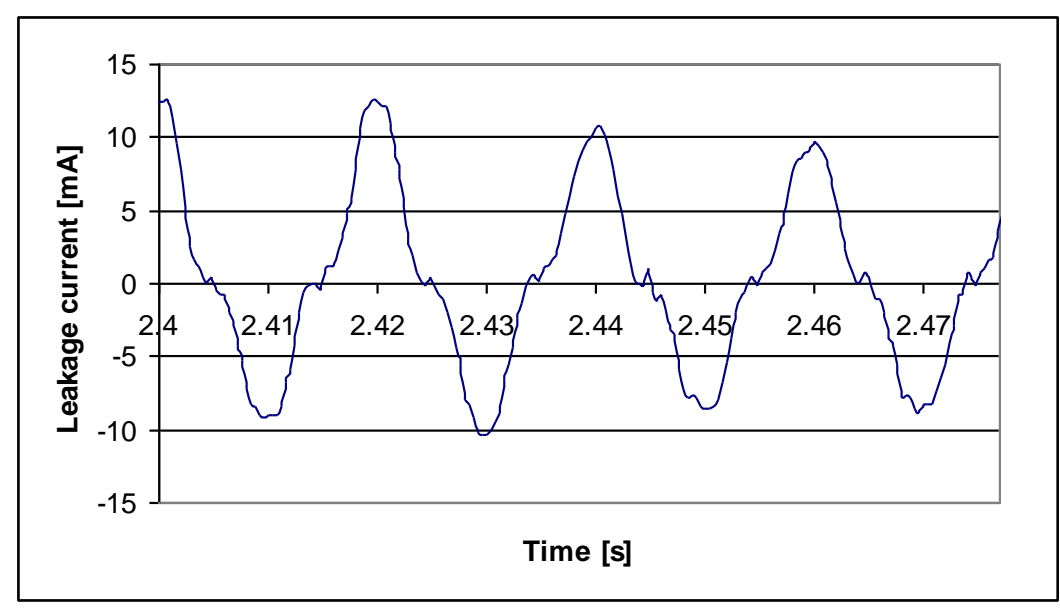

Figure 4.5: Leakage current $[\mathrm{mA}]$ waveform toward the end of the excitation phase 
Figure 4.6 shows the maximum peak currents recorded for all of the RTV SR coated glass samples using the OLCA. Channel 2 with a creepage distance of $277 \mathrm{~mm}$ has a peak current of $26 \mathrm{~mA}$ while channel 4 with a creepage distance of $346 \mathrm{~mm}$ has a peak current of $21.6 \mathrm{~mA}$. Channel 6 with a creepage distance of $433 \mathrm{~mm}$ has a peak current of $19.3 \mathrm{~mA}$. The discontinuity in Figure 4.6 shows an electrical power failure incidence. The spikes dropping the leakage current to zero $\mathrm{mA}$ indicate the times when the test were stopped, which occurred regularly to adjust the water level and conductivity of the solution. The RTV SR coated glass samples shows little or no effect when the water level decreases. This shows that a shorter creepage distance causes a higher leakage current.

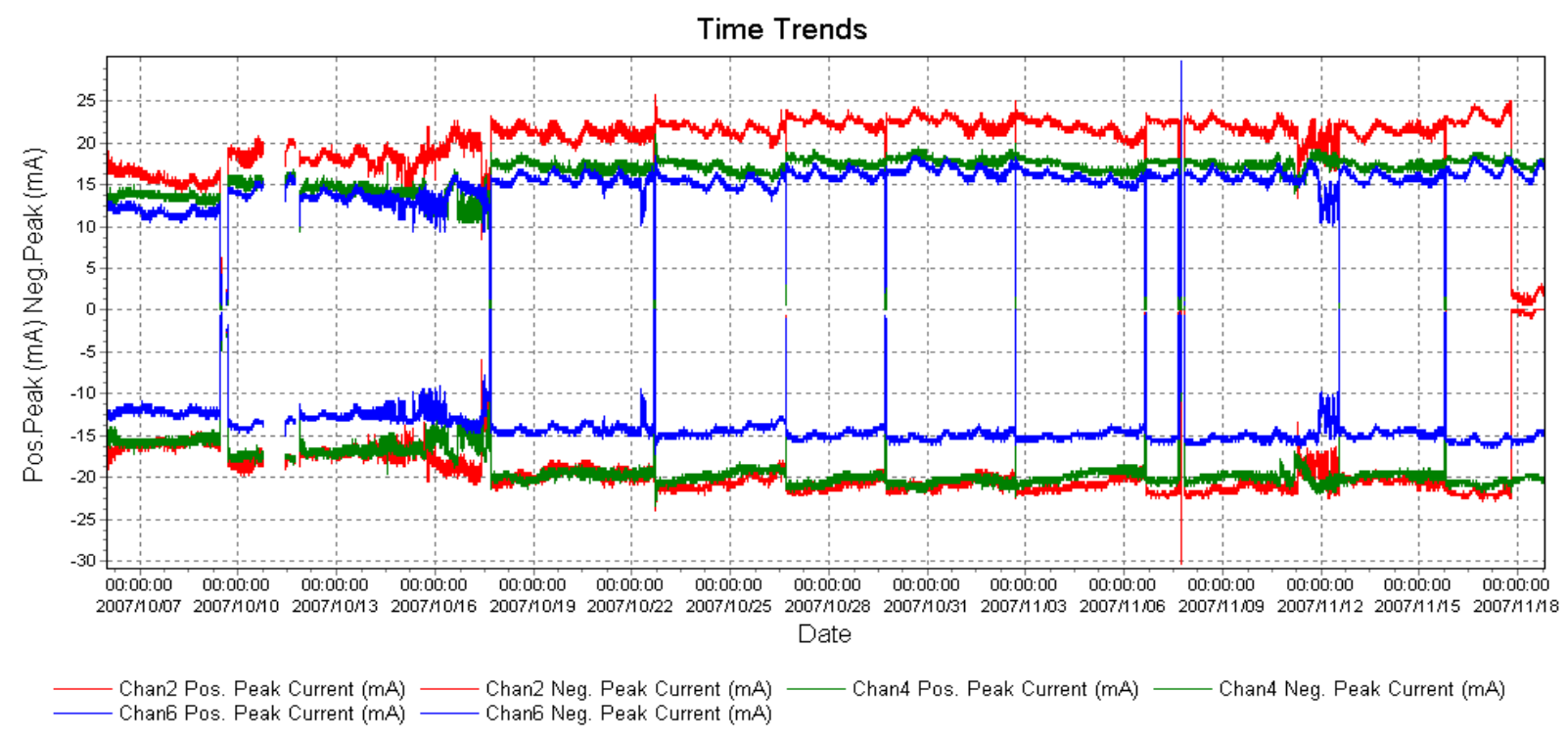

Figure 4.6: Peak leakage currents for the RTV SR coated glass samples

Figure 4.7 shows the peak leakage currents for the glass samples. Channel 1 with a creepage distance of $277 \mathrm{~mm}$ has a peak current of $29 \mathrm{~mA}$ while channel 3 with a creepage distance of $346 \mathrm{~mm}$ has a peak current of $20.2 \mathrm{~mA}$. Channel 5 with a creepage distance of $433 \mathrm{~mm}$ has a peak current of $15.2 \mathrm{~mA}$. Figure 4.7 also shows that the decreasing water level due to evaporation causes the leakage currents to decrease during each test run. The discontinuity shows when the power failure was experienced and the peak currents dropping to zero indicate the times when the test was stopped. This shows that a shorter creepage distance gives rise to a larger leakage current. 


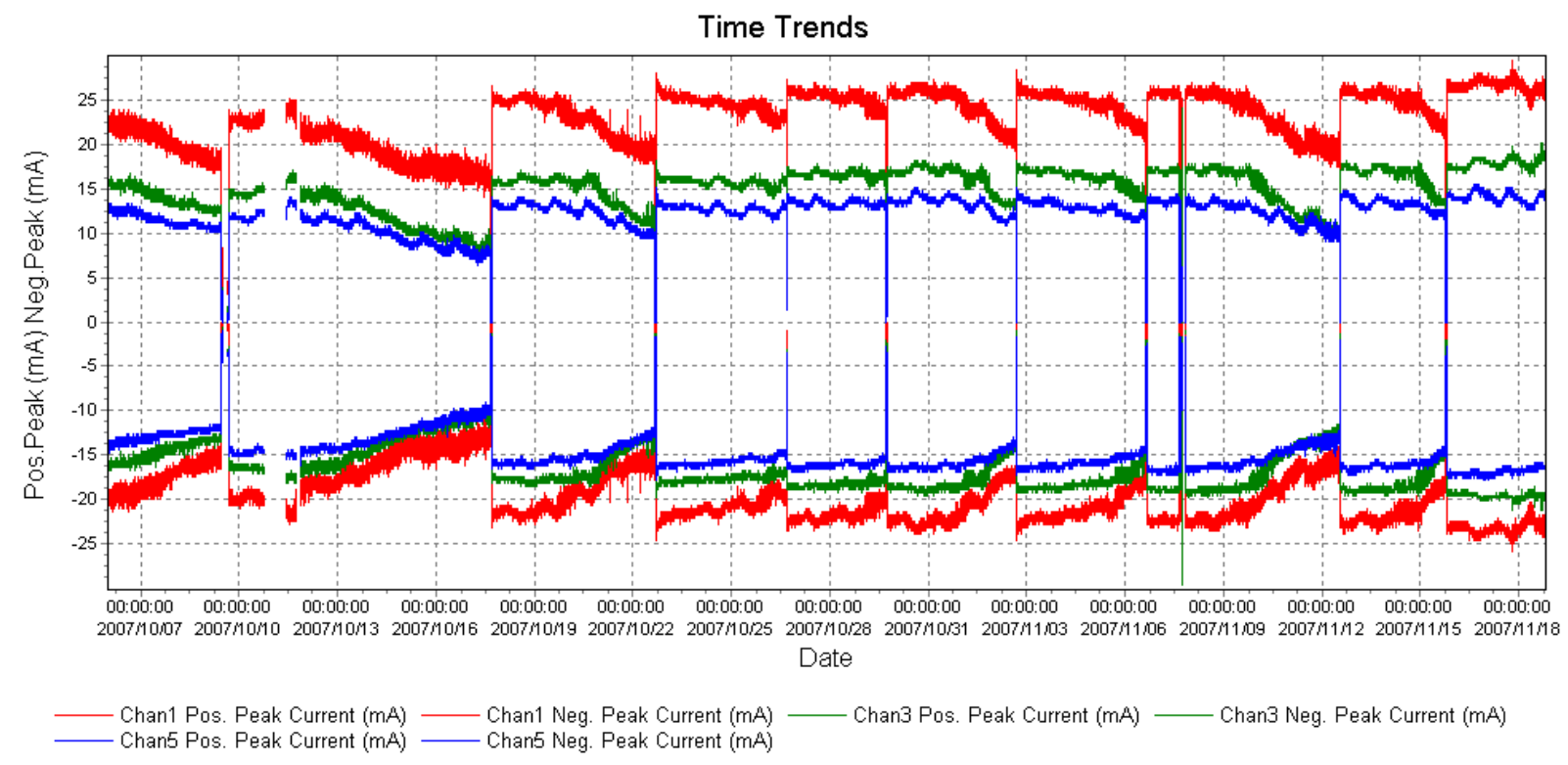

Figure 4.7: Peak leakage currents for the glass samples

Figure 4.8 compares the peak leakage currents for the two insulator rods with creepage distances of $277 \mathrm{~mm}$. Channel 1 represents a glass specimen while channel 2 represents RTV SR coated glass. The glass (Channel 1) has a peak current of 29 $\mathrm{mA}$ while the RTV SR coated class (Channel 2) has a peak current of $26 \mathrm{~mA}$. Figure 4.8 shows that glass (Channel 1) is heavily affected by the drop in water level, when compared to the RTV SR coated glass (Channel 2). This is shown by the reduction in the peak currents measured for channel 1 when the water level decreases. The step response in the results for channel 2 on 17 November is due to mechanical failure of the insulator rod fastenings, causing it to drop from the wheel. 


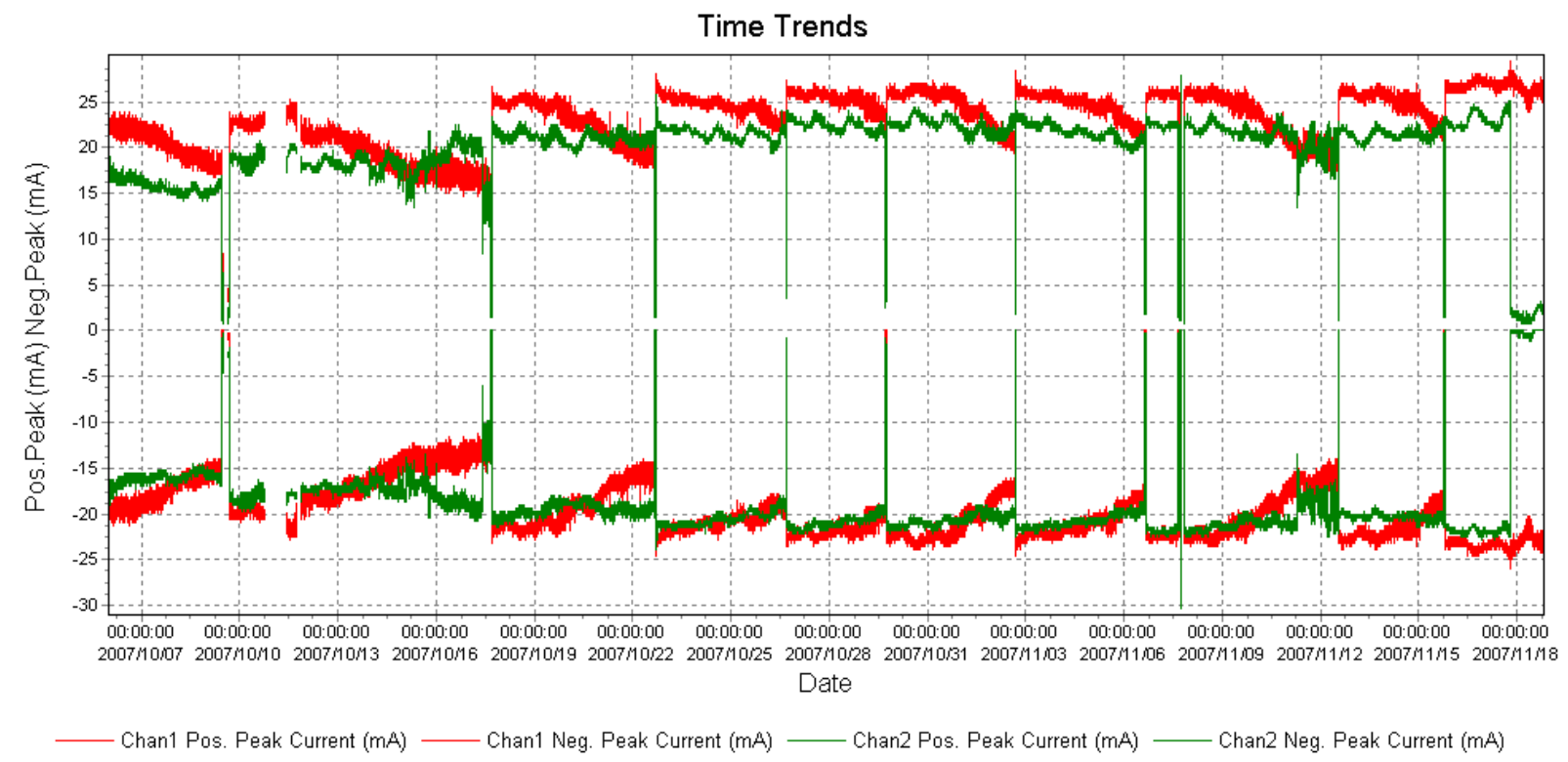

Figure 4.8: Peak leakage currents for the glass and RTV SR coated glass rods with a creepage distance of $277 \mathrm{~mm}$

Figure 4.9 shows the peak leakage currents for the two insulator rods with a creepage distance of $346 \mathrm{~mm}$. Channel 3 represents a glass specimen while channel 4 represents RTV SR coated glass. The glass (Channel 3) has a peak current of 20.2 $\mathrm{mA}$, while the RTV SR coated glass (Channel 4) has a peak current of $21.6 \mathrm{~mA}$. The leakage current for the glass decreases in peak current every time the water level decreases, while the leakage current for the RTV SR coated glass remains fairly constant.

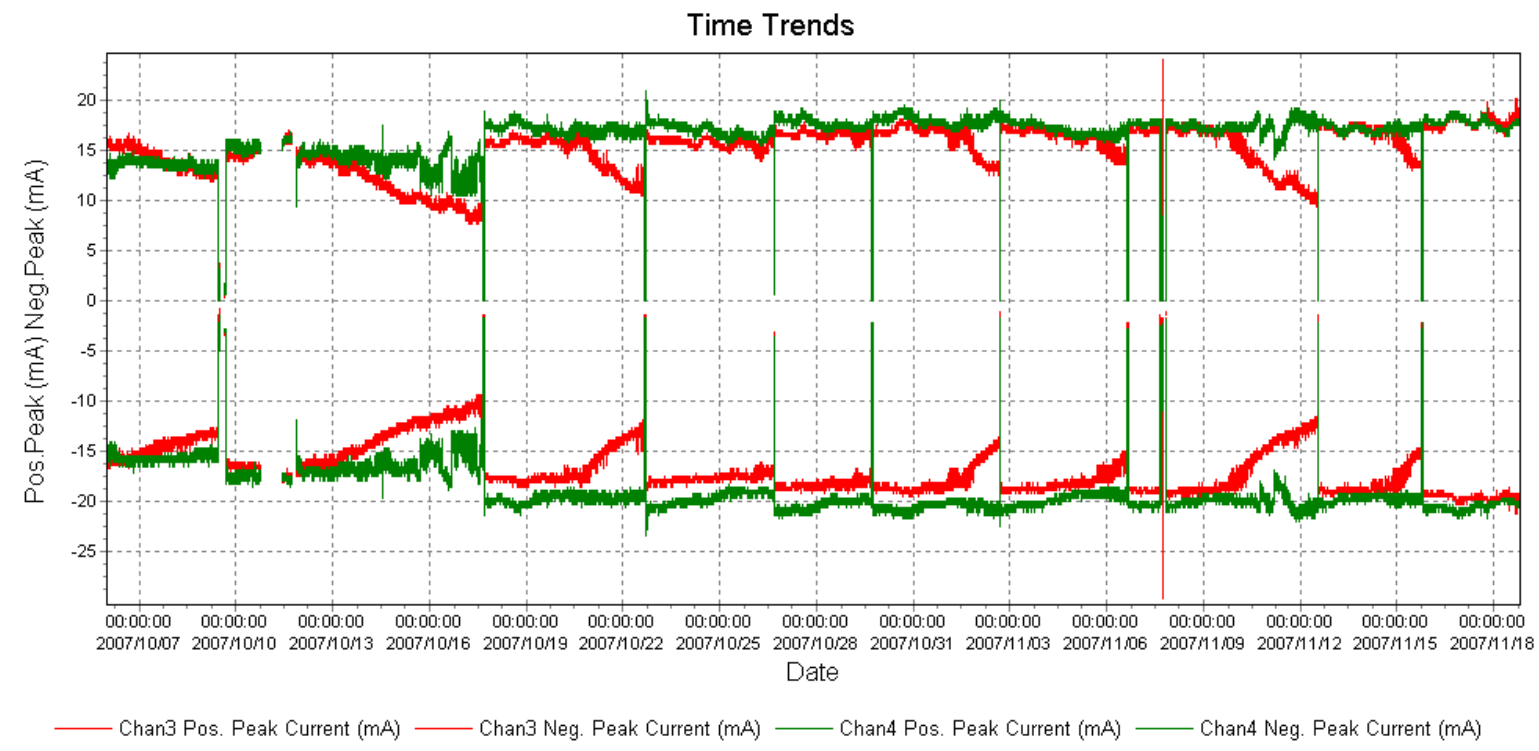

Figure 4.9: Peak leakage currents for the glass and RTV SR coated glass rods with a creepage distance of $346 \mathrm{~mm}$ 
Figure 4.10 shows the peak leakage currents for two insulator rods with a creepage distance of $433 \mathrm{~mm}$. Channel 5 represents a glass specimen while channel 6 represents RTV SR coated glass. The glass (Channel 5) has a peak current of 15.2 mA, while the RTV SR coated glass (Channel 6) has a peak current of $19.3 \mathrm{~mA}$. Figure 4.10 shows that the maximum value for the peak current belonging to the two channels is getting closer together towards the end. Glass (Channel 5) shows a decrease in the peak current whenever the level of the salt solution drops. The peak current for the RTV SR coated glass (Channel 6) remains fairly constant when the water level decreases.

The results shown in Figure 4.6 to Figure 4.10 indicate that there is a relationship between the salt-solution level, the conductivity and the peak current. Whenever the test was stopped, the following observations were made:

- An increase in conductivity value.

- A decrease in the specified salt-water level.

- A decrease in the maximum peak current.

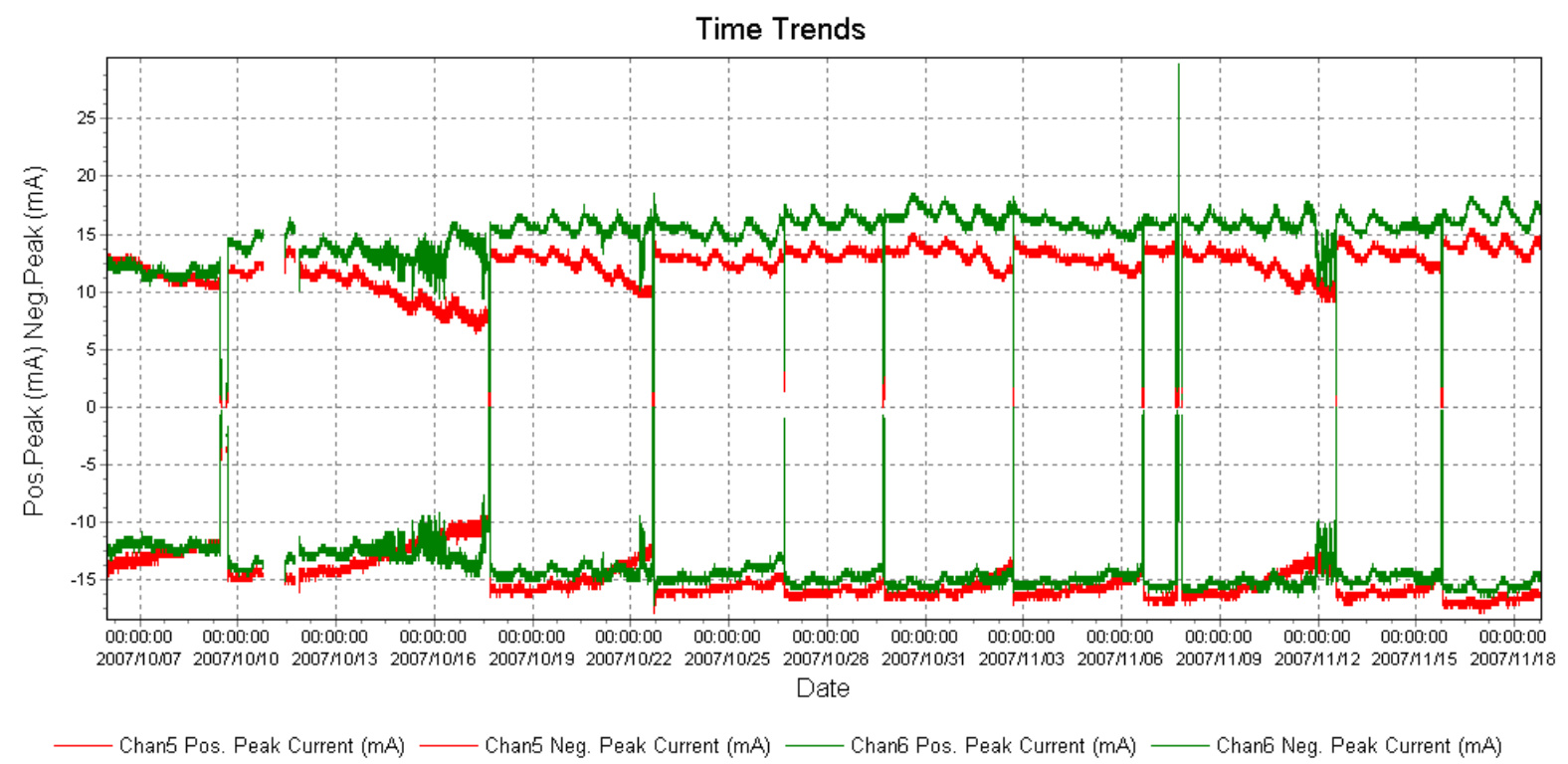

Figure 4.10: Peak leakage currents for the glass and RTV SR coated glass rods with a creepage distance of $433 \mathrm{~mm}$

\subsubsection{ESDD Results}

Surface deposit measurements were used to determine the pollution severity class. The surface deposit index is given by the Equivalent Salt Deposit Density (ESDD). 
Table 4.3 summarizes the ESDD results for the test samples. However, the ESDD value was found to represent a very light condition in terms of the pollution severity class is given in Appendix D.

Table 4.3: The relationship between volume conductivity and ESDD value

\begin{tabular}{|l|l|l|r|r|}
\hline $\begin{array}{l}\text { Channel } \\
\text { No. }\end{array}$ & $\begin{array}{l}\text { Volume of } \\
\text { Distilled Water } \\
{[\mathrm{ml}]}\end{array}$ & $\begin{array}{l}\text { Conductivity } \\
\text { of } \\
\text { Water } \\
{[\mu \mathrm{S} / \mathrm{cm}]}\end{array}$ & Volume Conductivity & $\begin{array}{l}\text { Temperature } \\
\text { of } \\
\text { the solution } \\
\left({ }^{\circ} \mathrm{C}\right)\end{array}$ \\
\hline 1 (Glass) & 1000 & 1.6 & 12.7 & 23.1 \\
\hline 2 (RTV SR) & 1000 & 2 & 9.2 & 23.6 \\
\hline 3 (Glass) & 1000 & 1.8 & 12.4 & 23.6 \\
\hline 4 (RTV SR) & 1000 & 1.8 & 20.5 & 22.9 \\
\hline 5 (Glass) & 1000 & 1.6 & 12.5 & 21.2 \\
\hline 6 (RTV SR) & 1000 & 1.6 & 16.5 & 21.2 \\
\hline
\end{tabular}

\subsubsection{Visual observations of aging}

At the end of the experiment, the six insulator rods were evaluated to determine how the materials aged with time. Visual inspections were conducted with the view to determine whether there are any signs of tracking or erosion on the rods. These results gave rise to a recommendation that real insulators instead of the insulator rods should be used in the second series of tests.

Figure 4.11 shows the glass samples that were used in the first test series, i.e. Channel $1($ Creepage distance $=277 \mathrm{~mm})$, Channel $3($ Creepage distance $=346 \mathrm{~mm})$, and Channel 5 (Creepage distance $=433 \mathrm{~mm}$ ). It is clearly shown that the glass samples did not age significantly with time. There were, however, brown discolorations on all of the samples. This discoloration was brought about by the combination of the slabs touching the high voltage wheel and then dipping the insulator rod into the specified salt-water solution. As time goes by, the water starts browning and this causes the insulators to also start browning. 


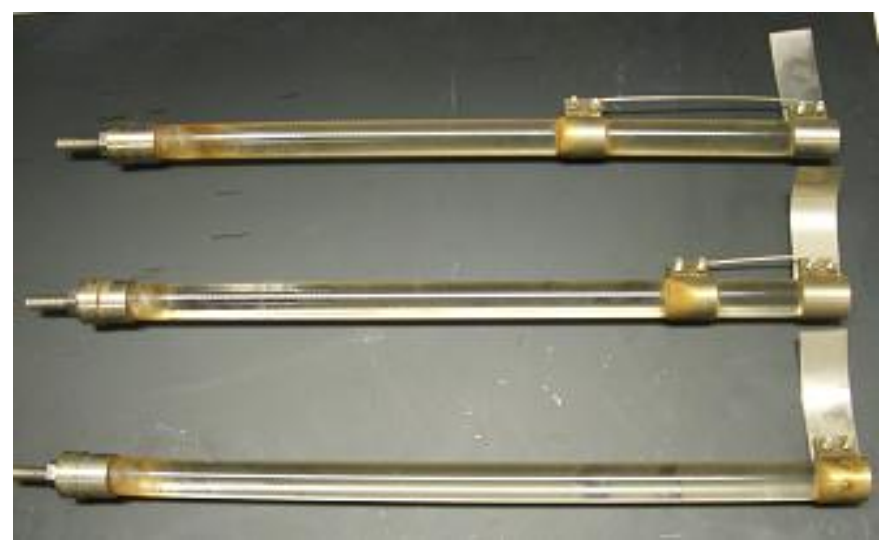

Figure 4.11: Visual aging of the glass samples

Figure 4.12 shows the RTV SR coated glass samples that were used in the first test series, i.e. Channel $2($ Creepage distance $=277 \mathrm{~mm})$, Channel $4($ Creepage distance $=$ $346 \mathrm{~mm})$, and Channel 6 (Creepage distance $=433 \mathrm{~mm})$. These samples also showed discoloration on all of the samples as well as signs of peeling.

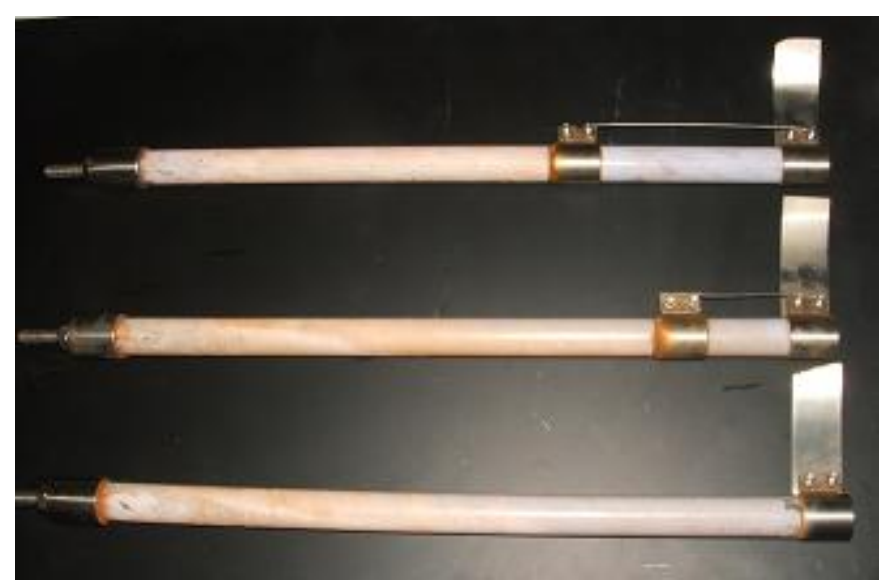

Figure 4.12: Visual aging of the RTV SR coated glass samples

Figure 4.13 shows the RTV SR coated glass insulator with a creepage distance of 277 $\mathrm{mm}$. This insulator shows signs of erosion (loss of material), as well as peeling of the coating.

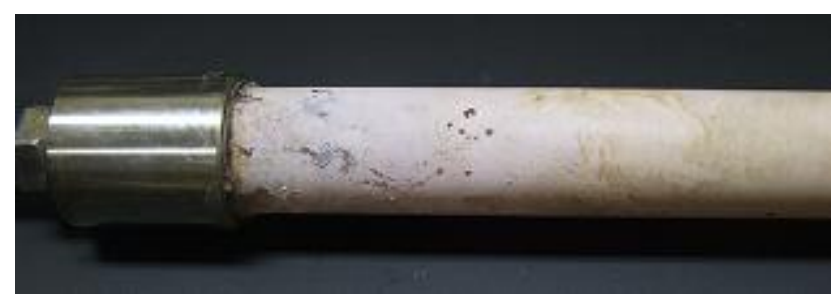

Figure 4.13: Visual aging of the RTV SR coated glass insulator with a creepage distance of $277 \mathrm{~mm}$ 
Figure 4.14 shows the RTV SR coated glass insulator with a creepage distance of 346 mm. This insulator shows very little or no signs of erosion and peeling, but there are small bubbles on the surface.

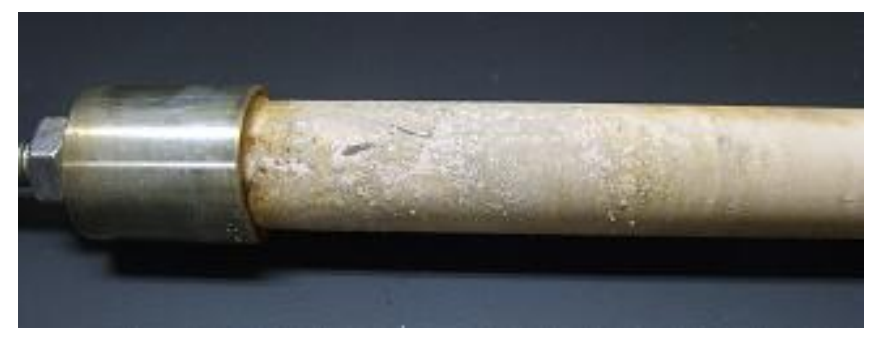

Figure 4.14: Visual aging of the RTV SR coated glass insulator with creepage distance of $346 \mathrm{~mm}$

Figure 4.15 shows the RTV SR coated glass insulator with a creepage distance of 433 mm. There is less peeling and erosion when compared to the insulator with a creepage distance of $277 \mathrm{~mm}$. There are also small little bubbles on the insulator coating, and it seems to be more than for the insulator with creepage distance of 346 $\mathrm{mm}$.

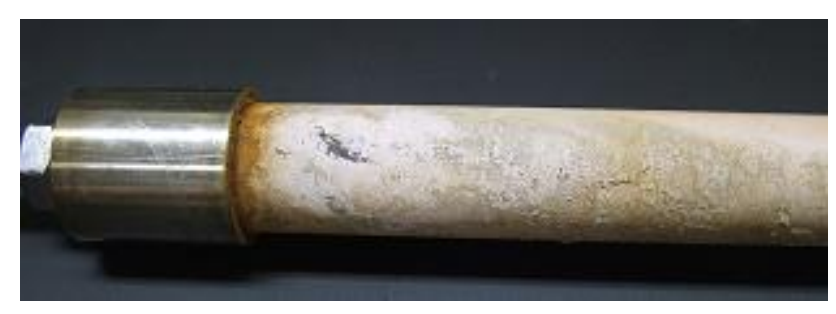

Figure 4.15: Visual aging of the RTV SR coated glass insulator with creepage distance of $433 \mathrm{~mm}$

\subsubsection{Hydrophobic properties}

The insulator rods were analysed to evaluate whether the insulators retained its hydrophobicity at the end of the test. Figure 4.16 show that the RTV SR coated glass sample with a creepage distance of $277 \mathrm{~mm}$ (Channel 2) has a wettability class of 4 , thus becoming hydrophillic. The insulator surface is thus still completely hydrophobic. Therefore the RTV SR coated glass sample with creepage distance of $277 \mathrm{~mm}$ almost became hydrophilic. 


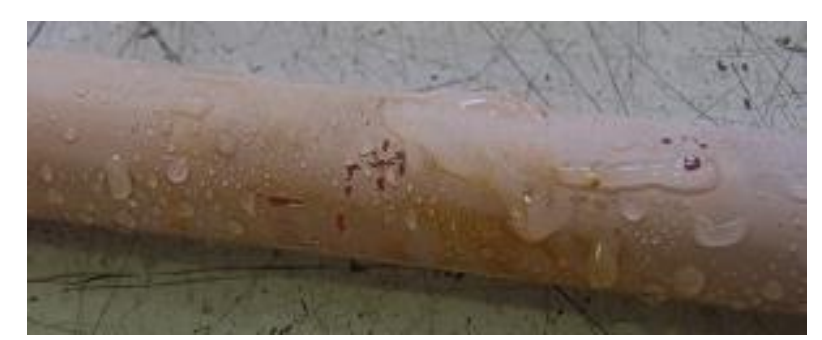

Figure 4.16: Wettability of the RTV SR coated glass sample with creepage distance of 277 mm (Channel 2)

Figure 4.17 shows that the RTV SR coated glass sample with a creepage distance of $346 \mathrm{~mm}$ (Channel 4) has a wettability class of 3. The insulator surface still hasn't lost its hydrophobicity and is in fact even better than the insulator with a creepage distance of $277 \mathrm{~mm}$.

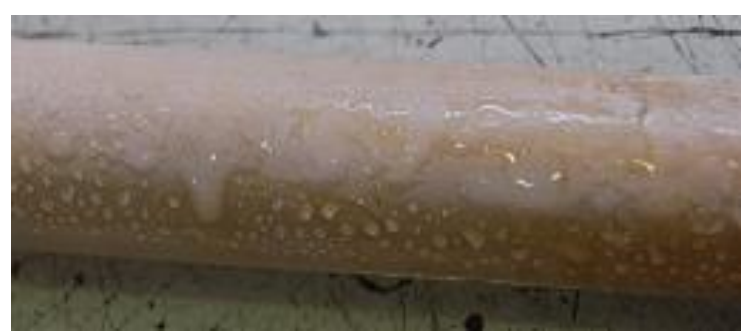

Figure 4.17: Wettability of the RTV SR coated glass sample with creepage distance of $346 \mathrm{~mm}$ (Channel 4)

Figure 4.18 shows that the RTV SR coated glass sample with creepage distance of $433 \mathrm{~mm}$ (Channel 6) has a wettability class of 1 . This insulator surface is still hydrophobic even though it has a wettability class lower than the insulator with a creepage distance of $346 \mathrm{~mm}$.

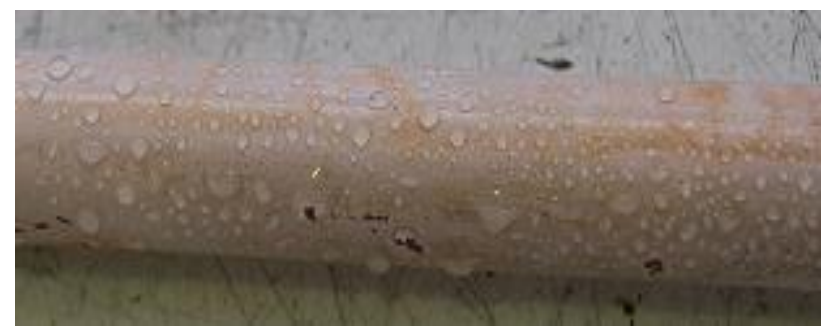

Figure 4.18: Wettability of the RTV SR coated glass sample with creepage distance of $433 \mathrm{~mm}$ (Channel 6)

Therefore, the RTV SR coated glass sample with a creepage distance of $433 \mathrm{~mm}$ (Channel 6) retains its hydrophobicity better, when compared to Channel 2 and Channel 4. 


\subsection{Second test series: HVAC excitation}

\subsubsection{Overview}

After the first Tracking Wheel Tester experiment was completed, a number of recommendations were made. This eventually led to the application of a combination of the IEC 61302 and the IEC 62217 standards. The first change that was made required that actual insulators be used in the tests, since the insulator rods did not give enough details on material aging, as well as performance in terms of leakage currents. The series of tests show that the water level and the water temperature affected the peak leakage currents significantly. A system was designed to regulate the water level automatically and a temperature controller had to be designed and incorporated for the second series of tests.

It was also concluded from the first experiment that the pollution severity class was very light and this required the test to go on for a longer period. This led to the suggestion of using the conductivity, as well as the excitation voltage level and creepage distance specified in the IEC 62217 standard, given in Appendix C.

A problem was encountered at the beginning of the experiment. The insulators were mounted on the stainless steel rotating wheel with the aluminium end fittings supplied by the manufacturer. However, the combination of the different metals such as stainless steel, aluminium, and galvanised steel, etc. with the salt-water solution led to the formation of a lasagna cell/battery. This problem was solved by replacing the different metal end fittings with stainless steel end fittings.

Figure 4.19 shows the rotating wheel with the six insulators as installed at the beginning of the second series of tests with HVAC excitation. 


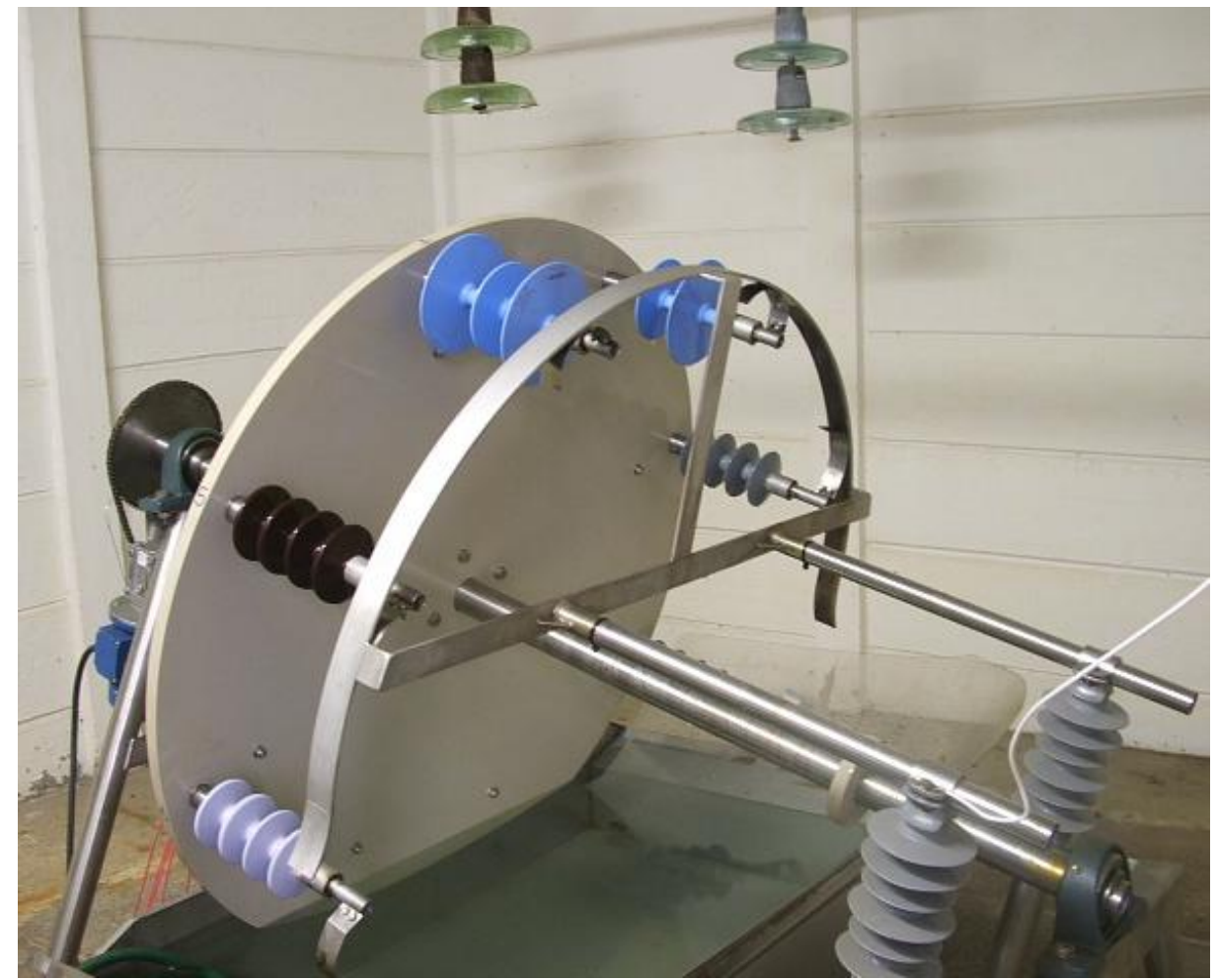

Figure 4.19: The Tracking Wheel Tester with the test samples used in the second series of tests with HVAC excitation.

\subsubsection{Test methodology and test procedures}

For the second series of tests, six different insulators from three different manufacturers, as summarized in Table 4.4, were installed on the rotating wheel. These test samples varied with regard to insulator material, Specific Creepage Distance (SCD), and insulator profile.

The tests were conducted for the following insulator materials:

- $\quad$ Ethylene propylene diene monomer (EPDM).

- $\quad$ High-temperature vulcanised Silicone rubber (HTV SR).

- Porcelain.

- $\quad$ Porcelain coated with Room-Temperature Vulcanised Silicone Rubber (RTV SR).

Two creepage distances were used, namely $380 \mathrm{~mm}$ and $476 \mathrm{~mm}$. After the metal end fittings of the insulators were replaced, the creepage distances given in Table 4.4 were obtained. Since the experiment was designed for a light pollution severity class, it was decided that a Unified Specific Creepage Distance (USCD) of $28 \mathrm{~mm} / \mathrm{kV}$ should be used. The excitation voltage was calculated from the relationship 


$$
\mathrm{SCD}=\frac{L C D}{\left(U_{m} / \sqrt{3}\right)}\left[\frac{m m}{k V}\right]
$$

where LCD denotes the total insulator creepage distance $[\mathrm{mm}], U_{m}$ denotes the maximum rms phase-to-phase system voltage $[\mathrm{kV}]$ and SCDdenotes the specific creepage distance $[\mathrm{mm} / \mathrm{kV}]$. This yields $13.6 \mathrm{kV}$, the actual voltage across the insulator for the creepage distance of $380 \mathrm{~mm}$ and the Unified Specific Creepage distance for a light pollution severity class, i.e. $28 \mathrm{~mm} / \mathrm{kV}$.

For the HTV SR insulator with a Unified Specific Creepage Distance of $35 \mathrm{~mm} / \mathrm{kV}$ given in Table 4.4, multiplying the applied voltage of $13.6 \mathrm{kV}$ with the Unified Specific Creepage Distance for the medium pollution severity class, yields a creepage distance of $476 \mathrm{~mm}$. This creepage distance is then used for the insulator on channel 4. The conductivity value used in this experiment was different from the first test series. i.e. the salinity of the specified salt-water solution was specified as 1.40 $\left[\mathrm{kg} / \mathrm{m}^{3}\right] \pm 0.06\left[\mathrm{~kg} / \mathrm{m}^{3}\right]$ as given in IEC 62217 . The conductivity of the specified salt-water solution at $20{ }^{\circ} \mathrm{C}$ was determined using the formula given in equation 4.2 , yielding $0.243[\mathrm{~S} / \mathrm{m}] @ 20{ }^{\circ} \mathrm{C}[24]$.

$$
\mathrm{S}_{a}=\left[5.7 \times \sigma_{20}\right]^{1.03}
$$

where $\sigma_{20}$ denotes the volume conductivity corrected to $20{ }^{\circ} \mathrm{C}$ in $[\mathrm{S} / \mathrm{m}]$ and $\mathrm{S}_{a}$ denotes the salinity of the salt-water solution in $\left[\mathrm{kg} / \mathrm{m}^{3}\right]$.

Table 4.4: Summary of test samples for the second series of tests with HVAC excitation.

\begin{tabular}{|l|l|l|l|l|}
\hline Insulator Name & Type of Material & $\begin{array}{l}\text { Manufacturer } \\
\text { Name }\end{array}$ & $\begin{array}{l}\text { Creepage } \\
\text { Distance } \\
{[\mathbf{m m}]}\end{array}$ & $\begin{array}{l}\text { Unified Specific } \\
\text { Creepage } \\
\text { Distance } \\
{\left[\mathbf{m m} / \mathbf{k} \mathbf{V}_{p g}\right]}\end{array}$ \\
\hline Channel 1 & EPDM & $(\mathrm{A})$ & 380 & 28 \\
\hline Channel 2 & HTV SR & (A) & 380 & 28 \\
\hline Channel 3 & HTV SR & (B) & 380 & 28 \\
\hline Channel 4 & HTV SR & (B) & 476 & 35 \\
\hline Channel 5 & Porcelain & (C) & 380 & 28 \\
\hline Channel 6 & $\begin{array}{l}\text { RTV SR coated } \\
\text { porcelain }\end{array}$ & (D) & 380 & 28 \\
\hline
\end{tabular}

The same start-up operating procedure as used in the first series of tests was applied, except that the variac had to be adjusted until a voltage of $13.6 \mathrm{kV}$ was obtained on the HV side of the transformer. The conductivity value had to be maintained at 0.243 
$\pm 0.114[\mathrm{mS} / \mathrm{cm}] @ 20{ }^{\circ} \mathrm{C}$. The temperature specified for the conductivity value was given as $20{ }^{\circ} \mathrm{C} \pm 5 \mathrm{~K}$.

\subsubsection{Summary of test results}

\subsubsection{Leakage currents}

Table 4.5 and Figure 4.20 summarize the peak leakage current results for the EPDM (Channel 1) and HTV SR (Channel 2) insulators with unified SCD of $28 \mathrm{~mm} / \mathrm{kV}$. These two insulators have the same manufacturer, profile, and SCD, but represent different materials. At the beginning of the experiment the HTV SR insulator (Channel 2) had a peak current of $42 \mathrm{~mA}$ while the EPDM insulator (Channel 1) had a peak current $45 \mathrm{~mA}$. However, as the hydrophobic property of the HTV SR insulator (Channel 2) was lost, the HTV SR insulator exhibited higher peak leakage currents compared to the EPDM insulator (Channel 1). Towards the end of the test, HTV SR insulator (Channel 2) stabilised at approximately $61 \mathrm{~mA}$, while EPDM insulator (Channel 1) stabilised at approximately $58 \mathrm{~mA}$. Thus, towards the end of the test EPDM insulator performed better than HTV SR insulator in terms of peak leakage current.

The following observations apply for the leakage current profile shown in Figure 4.20:

- Incidences when the peak currents drop to zero indicate when the test was stopped for pictures to be taken or to fix maintenance problems.

- The space or break in between the $27^{\text {th }}$ and the $28^{\text {th }}$ April 2008 indicates the time when the OLCA did not record the peak currents, because the OLCA memory was full.

- A break also indicates a period during which a power blackout was experienced.

- $\quad$ The red spikes shown for Channel 1 on the $11^{\text {th }}$ May 2008 indicates a period during which the HV voltage transfer brush malfunctioned, and thus the insulator made intermittent contact with the applied voltage. The brush was replaced on the $12^{\text {th }}$ May 2008.

In this particular experiment, it was discovered, that in the beginning of the experiment, the HTV SR insulator (Channel 2) performed better than the EPDM insulator (Channel 1), provided the hydrophobicity was not lost. However, once the 
hydrophobicity was lost, then the EPDM insulator (Channel 1) performed better than the HTV SR insulator (Channel 2).

Table 4.5: Peak leakage currents for Channel 1 and Channel 2 for HVAC excitation

\begin{tabular}{|l|l|l|}
\hline $\begin{array}{l}\text { Peak current }[\mathrm{mA}] \text { in the } \\
\text { beginning }\end{array}$ & $45 \mathrm{~mA}$ Channel 2 (HTV SR) \\
\hline Peak current [mA] in the end & $58 \mathrm{~mA}$ & $42 \mathrm{~mA}$ \\
\hline
\end{tabular}

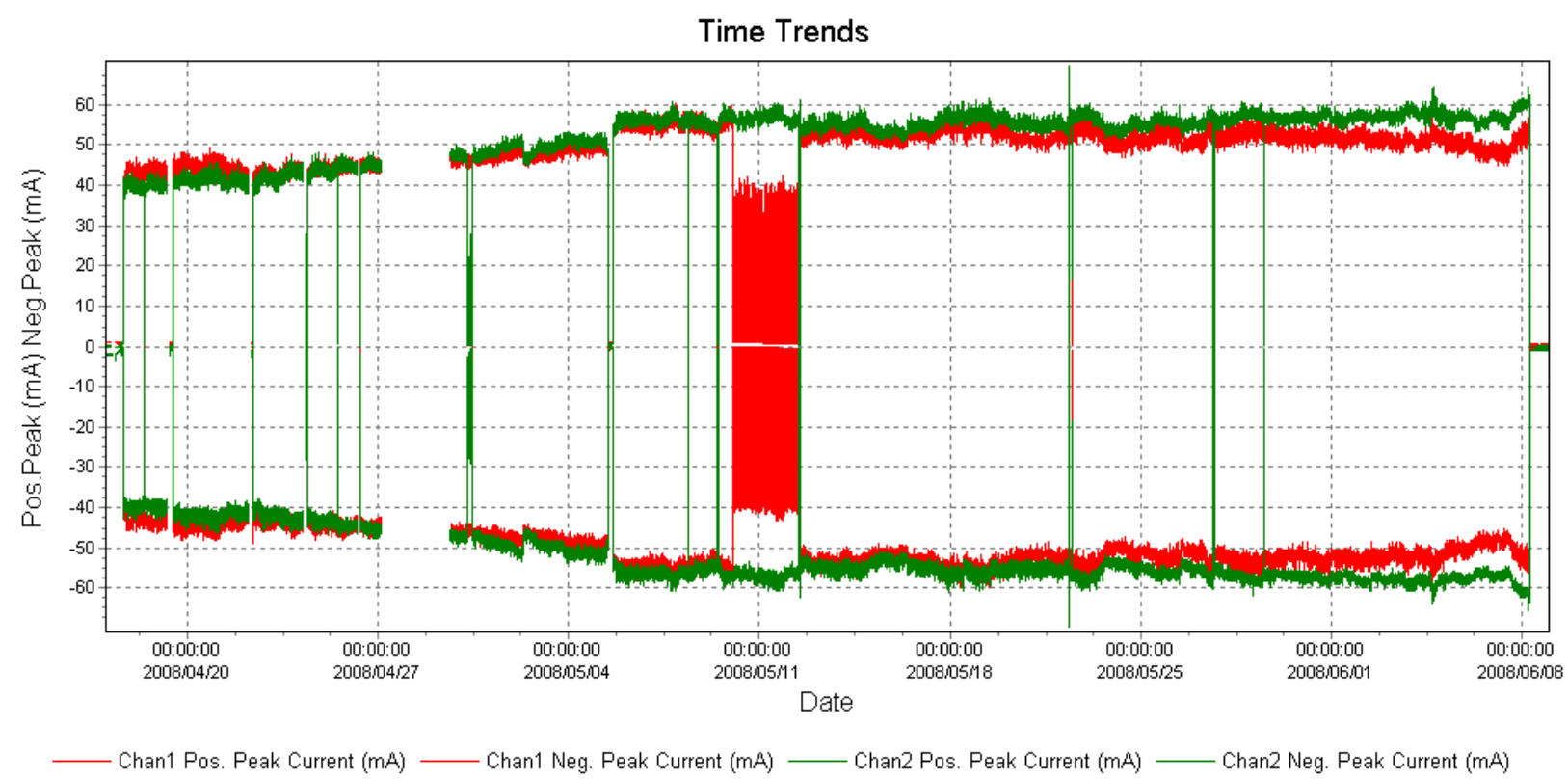

Figure 4.20: Peak leakage currents for the EPDM and HTV SR insulators for HVAC excitation

Table 4.6 and Figure 4.21 summarize the peak leakage current results for the HTV SR insulators with a unified SCD of $28 \mathrm{~mm} / \mathrm{kV}$ (Channel 3) and $35 \mathrm{~mm} / \mathrm{kV}$ (Channel 4) respectively. At the beginning of the experiment the HTV SR insulator with unified SCD of $28 \mathrm{~mm} / \mathrm{kV}$ (Channel 3) had a peak current of $40 \mathrm{~mA}$ while the HTV SR insulator with a unified SCD of $35 \mathrm{~mm} / \mathrm{kV}$ (Channel 4) had a peak current of approximately $30 \mathrm{~mA}$.

On the $14^{\text {th }}$ May 2008 a sharp rise in peak currents was experienced by the HTV SR insulator with SCD of $28 \mathrm{~mm} / \mathrm{kV}$ (Channel 3). A puncture, shown in Figure 4.22, developed on this insulator on the $17^{\text {th }}$ May 2008, causing a further rise in peak current until the insulator tripped. The peak current for the HTV SR with SCD of 35 $\mathrm{mm} / \mathrm{kV}$ (Channel 4) rised very slowly to $55 \mathrm{~mA}$ at the end of the test. In terms of leakage current, the HTV SR insulator with SCD of $28 \mathrm{~mm} / \mathrm{kV}$ (Channel 3) performed worse than the HTV SR insulator with SCD of $35 \mathrm{~mm} / \mathrm{kV}$ (Channel 4). 
Table 4.6: Peak leakage currents for Channel 3 and Channel 4 for HVAC excitation

\begin{tabular}{|l|l|l|}
\hline & $\begin{array}{l}\text { Channel 3 (USCD of 28 } \\
\mathbf{m m} / \mathbf{k V} \text { ) }\end{array}$ & $\begin{array}{l}\text { Channel 4 (USCDof 35 } \\
\mathbf{m m} / \mathbf{k V} \text { ) }\end{array}$ \\
\hline $\begin{array}{l}\text { Peak current [mA] in the } \\
\text { beginning }\end{array}$ & $40 \mathrm{~mA}$ & $30 \mathrm{~mA}$ \\
\hline Peak current $[\mathrm{mA}]$ in the end & $\begin{array}{l}\text { Puncture ocurred } \\
>60 \mathrm{~mA}\end{array}$ & $55 \mathrm{~mA}$ \\
\hline
\end{tabular}

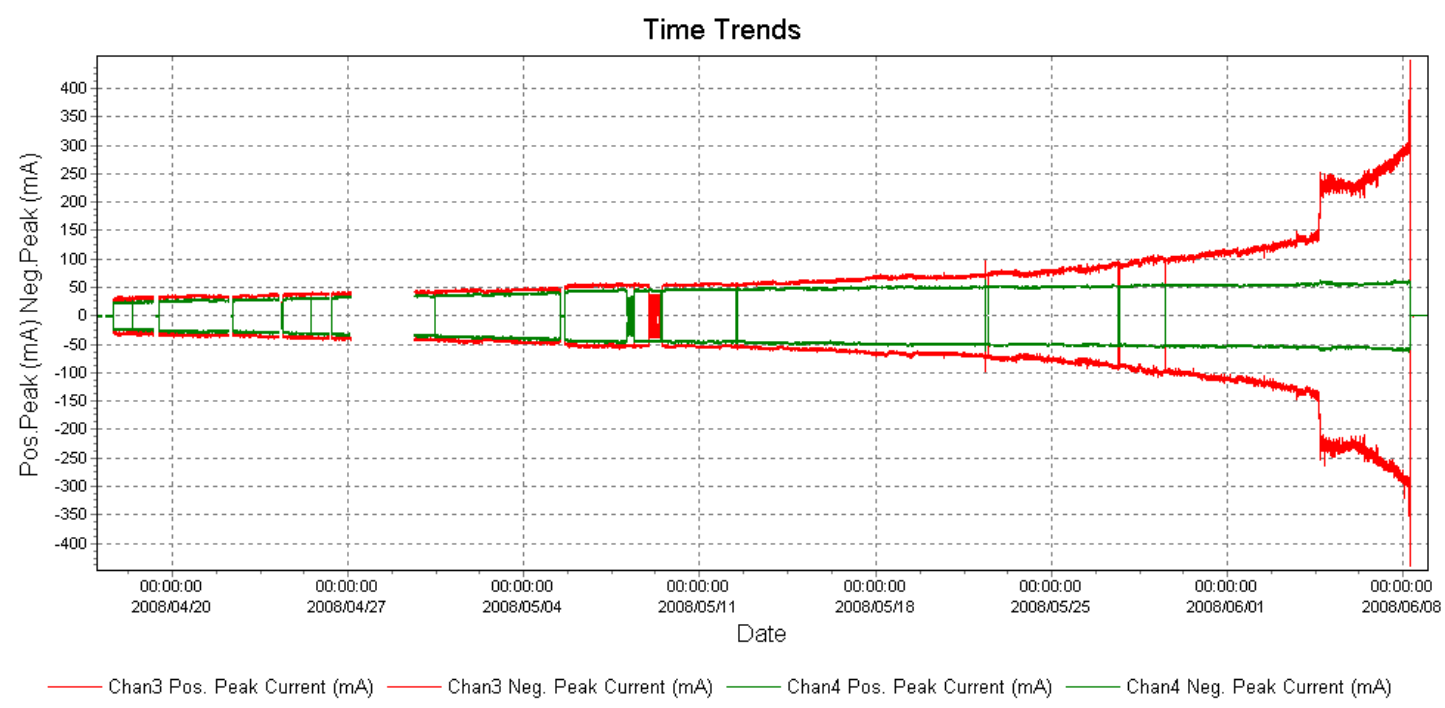

Figure 4.21: Peak leakage currents for the two HTV SR insulators with different SCD for HVAC excitation

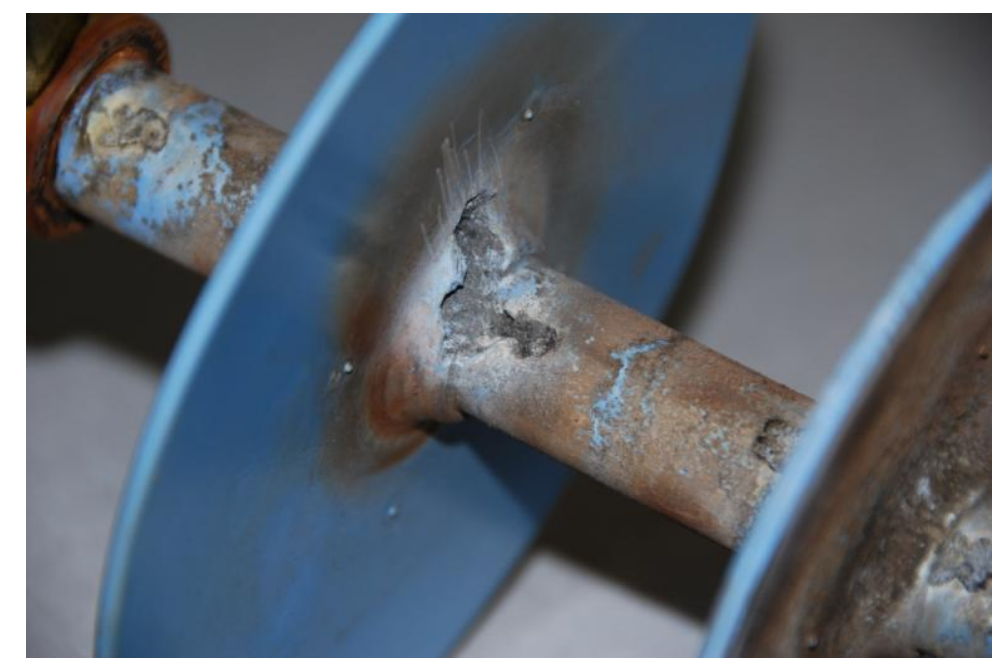

Figure 4.22: The puncture on Channel 3 (HTV SR insulator with SCD of $28 \mathrm{~mm} / \mathrm{kV}$ ) for HVAC excitation

Table 4.7 and Figure 4.23 summarize the leakage current results for the porcelain insulator (Channel 5) and the RTV SR coated porcelain insulator (Channel 6). At the beginning of the experiment, the porcelain insulator (Channel 5) had a peak current of 
$50 \mathrm{~mA}$ while the RTV SR coated porcelain insulator (Channel 6) had a peak current of $60 \mathrm{~mA}$. For the first two weeks, both test samples exhibited stable peak currents. As the test proceeded, however, the RTV SR coated sample (Channel 6) experienced an increase peak current and kept on rising until a stable value of $222 \mathrm{~mA}$ was reached, and then oscillated around this value until the end of the test. The reference porcelain insulator (Channel 5) stabilised at approximately $60 \mathrm{~mA}$, while the rise to the $60 \mathrm{~mA}$ was very slow. This particular test was stopped on the $8^{\text {th }}$ June 2008 , when the HTV SR insulator with SCDof $28 \mathrm{~mm} / \mathrm{kV}$ (Channel 3) insulator tripped. In terms of leakage current, the RTV SR coated porcelain (Channel 6) performed worse than the uncoated porcelain insulator (Channel 5), once its hydrophobicity was lost.

Table 4.7: Peak leakage currents for Channel 5 and Channel 6 for HVAC excitation

\begin{tabular}{|l|l|l|}
\hline & Channel 5 ( Porcelain) & $\begin{array}{l}\text { Channel 6 (RTV SR coated } \\
\text { porcelain) }\end{array}$ \\
\hline $\begin{array}{l}\text { Peak current [mA] in the } \\
\text { beginning }\end{array}$ & $50 \mathrm{~mA}$ & $60 \mathrm{~mA}$ \\
\hline Peak current $[\mathrm{mA}]$ in the end & $60 \mathrm{~mA}$ & $222 \mathrm{~mA}$ \\
\hline
\end{tabular}

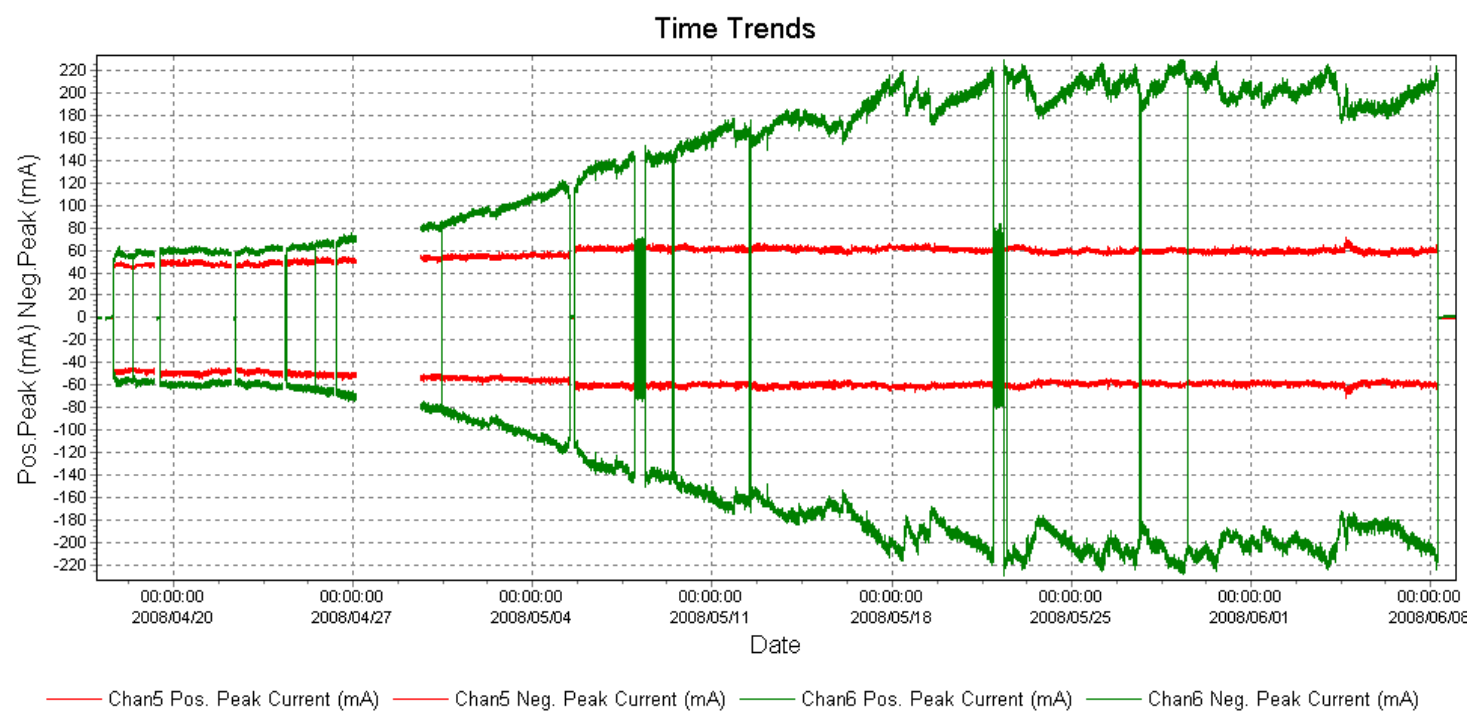

Figure 4.23: Peak leakage currents for the uncoated porcelain and RTV SR coated porcelain insulators for HVAC excitation

\subsubsection{Visual observations of aging}

Visual observations were made on the test insulators on a daily basis with the view to determine how the insulators aged with time. Photographs and video recordings of the discharges of the insulators were taken on weekly basis.

Figure 4.24 shows the EPDM insulator with a unified Specific Creepage Distance of $28 \mathrm{~mm} / \mathrm{kV}$ (Channel 1) before and after the experiment, while Figure 4.25 shows the 
aging in more detail. There is a browning discoloration and loss of material or erosion on the insulator. There is also widening and small little brown bubbles across the mould lines on the insulator a clear circling between the bottom sheds and the rods as illustrated in Figure 4.25.
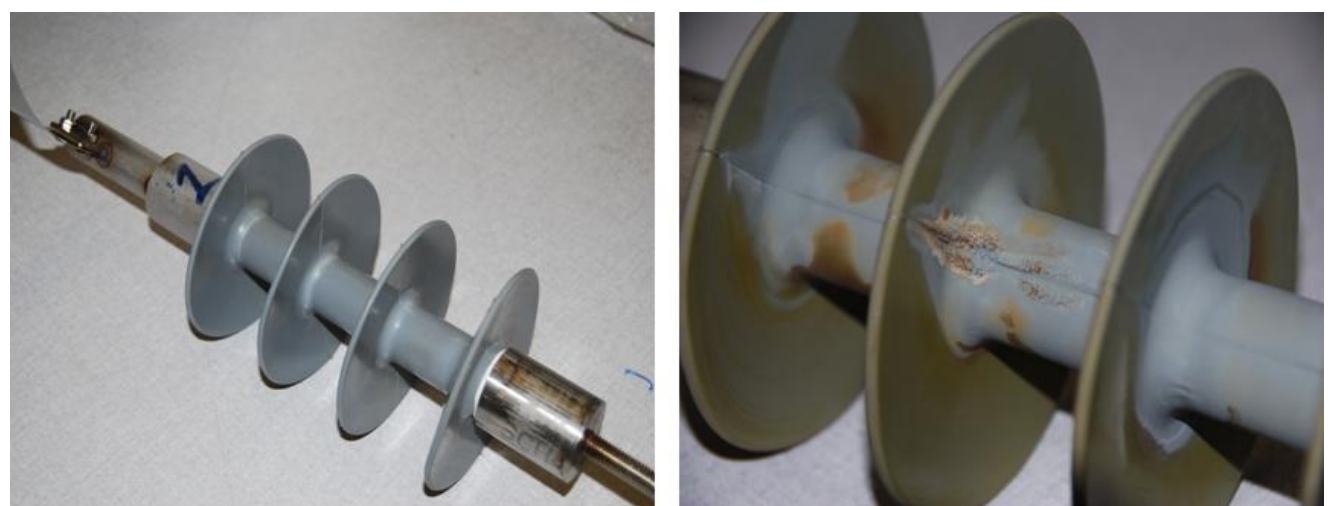

Figure 4.24: The EPDM insulator (Channel 1) before and after the test for HVAC excitation

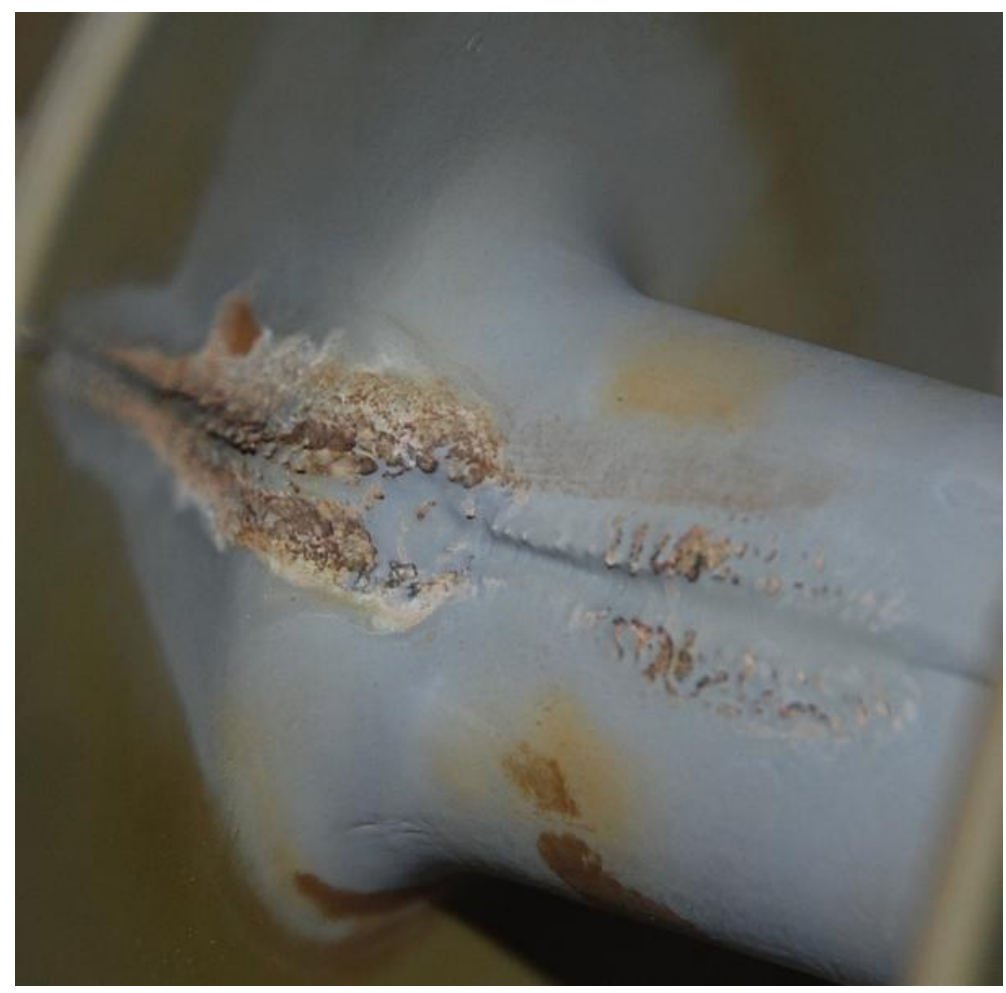

Figure 4.25: Detailed view of aging of the EPDM insulator (Channel 1) for HVAC excitation

Figure 4.26 shows the HTV SR insulator with a unified Specific Creepage Distance of SCDof $28 \mathrm{~mm} / \mathrm{kV}$ (Channel 2) before and after the experiment, while Figure 4.27 shows the aging in more detail. There are small signs of tracking on the pollution layer and darkening, as well as clearing on the mould lines. The insulator also has a 
brown discoloration, which is mostly on the rods, even though there browning on the sheds as well.
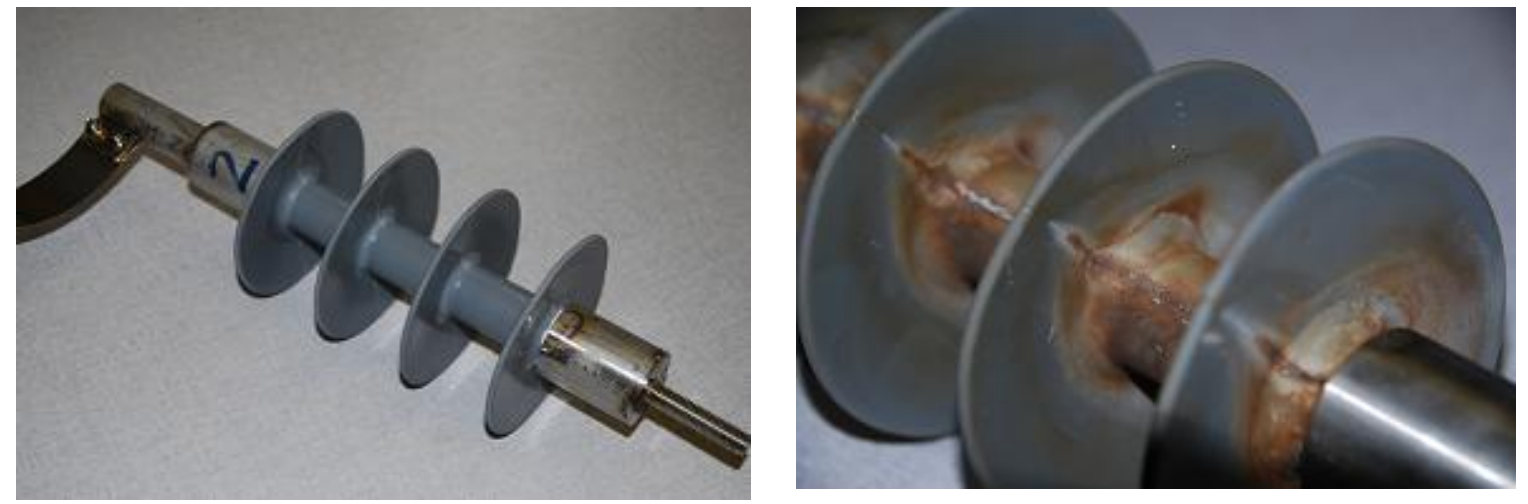

Figure 4.26: The HTV SR insulator (Channel 2) before and after the test for HVAC excitation

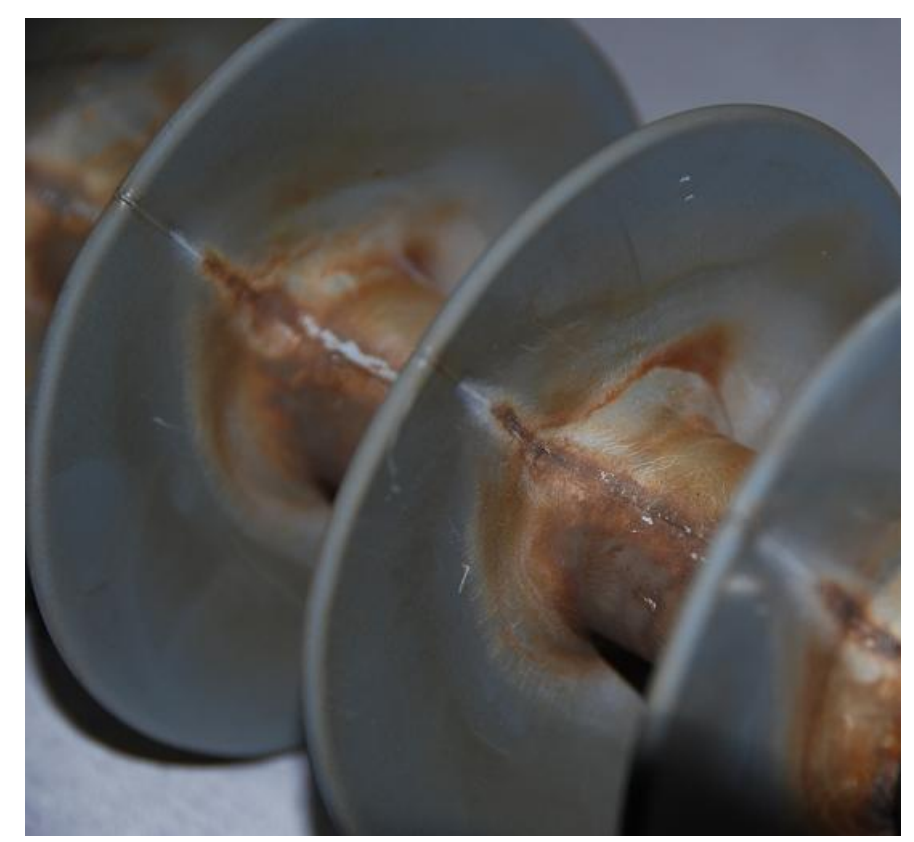

Figure 4.27: Detailed view of aging of the HTV SR insulator (Channel 2) for HVAC excitation

Figure 4.28 shows the EPDM (Channel 1) and HTV SR (Channel 2) insulators. Both insulators had discoloration. The EPDM insulator, however, had loss of material or erosion and widening and small brown bubbles across the mould lines on the insulator. The HTV SR had small signs of tracking on the pollution layer, and darkening as well as clearing on the mould lines. Thus, it was hard to determine which material aged faster, since the aging mechanism was different. 

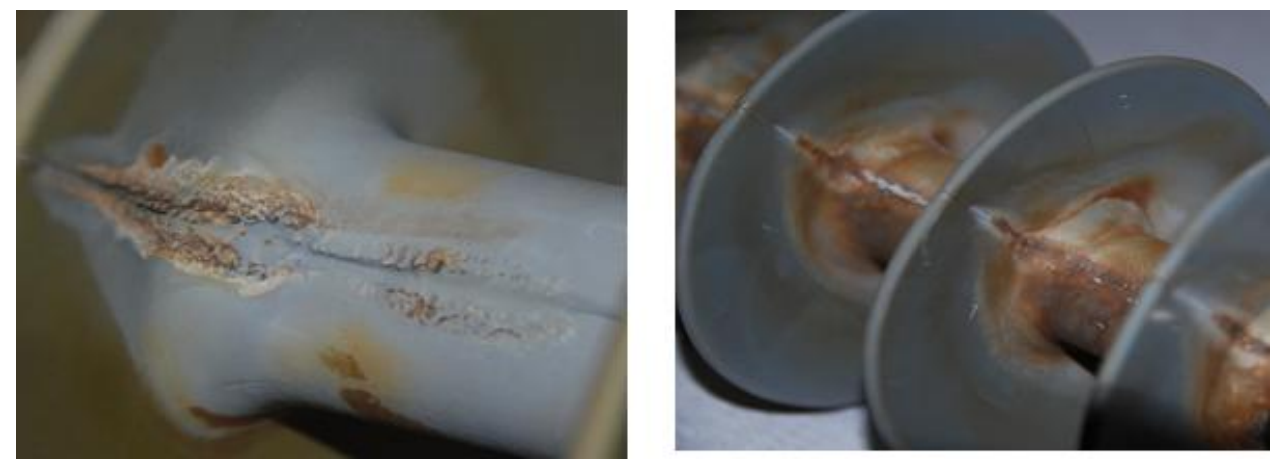

Figure 4.28 Comparison of aging of the EPDM and HTV SR insulators for HVAC excitation Figure 4.29 shows the HTV SR insulator with a unified Specific Creepage Distance of $28 \mathrm{~mm} / \mathrm{kV}$ (Channel 3) before and after the experiment, while Figure 4.30 shows the aging in more detail. It developed five punctures on the sheds and rods, with white solid deposit on the punctures. There are small signs of browning or darkening at the tip of the metal end fitting and it also shows discoloration on the insulator. Furthermore, signs of tracking are evident.
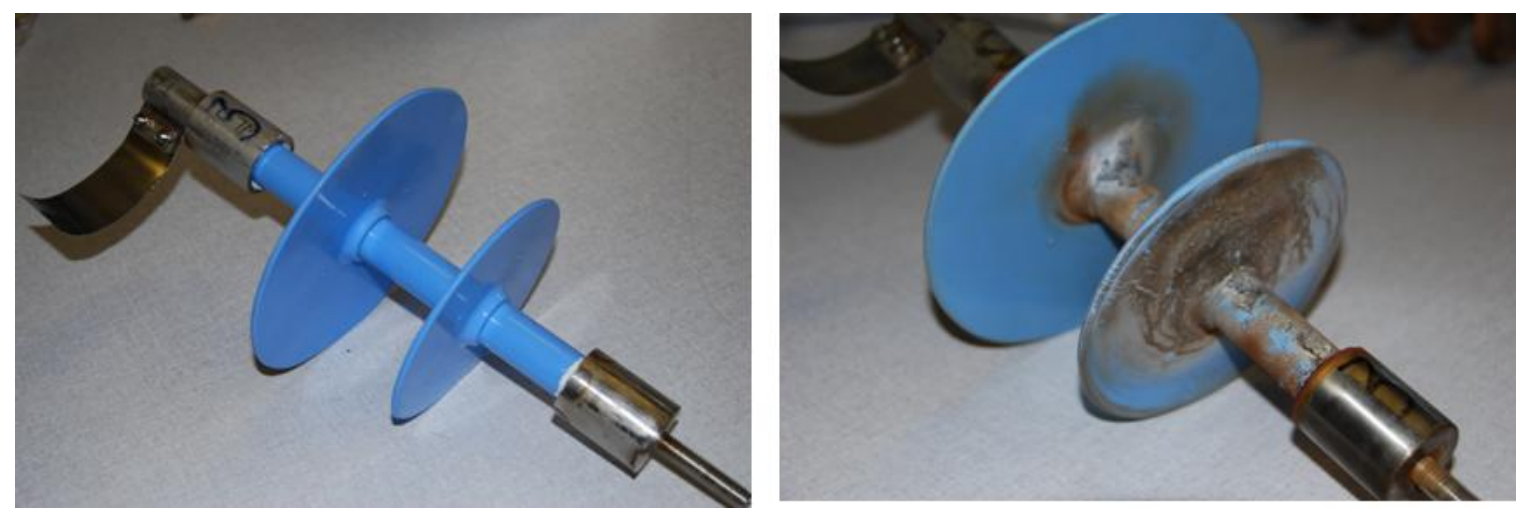

Figure 4.29: HTV SR insulator (Channel 3) before and after the test for HVAC excitation

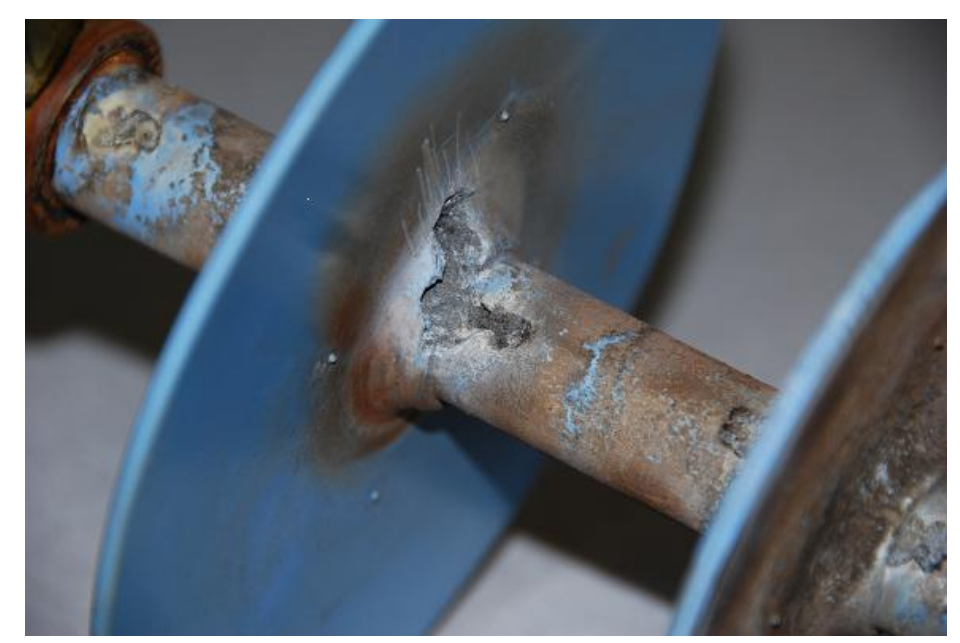

Figure 4.30: Detailed view of aging of the HTV SR insulator (Channel 3) for HVAC excitation 
Figure 4.31show the HTV SR insulators with unified SCD of $28 \mathrm{~mm} / \mathrm{kV}$ from manufacturer A (Channel 2) and manufacturer B (Channel 3) respectively. The insulator from manufacturer $\mathrm{A}$ had brown discoloration and small tracks on the pollution layer. The insulator from manufacturer B has five punctures and small tracks on the pollution layer, with severe erosion. In this particular case, the insulator from Manufacturer A performed better compared to the insulator from manufacturer $\mathrm{B}$ in terms of material aging.
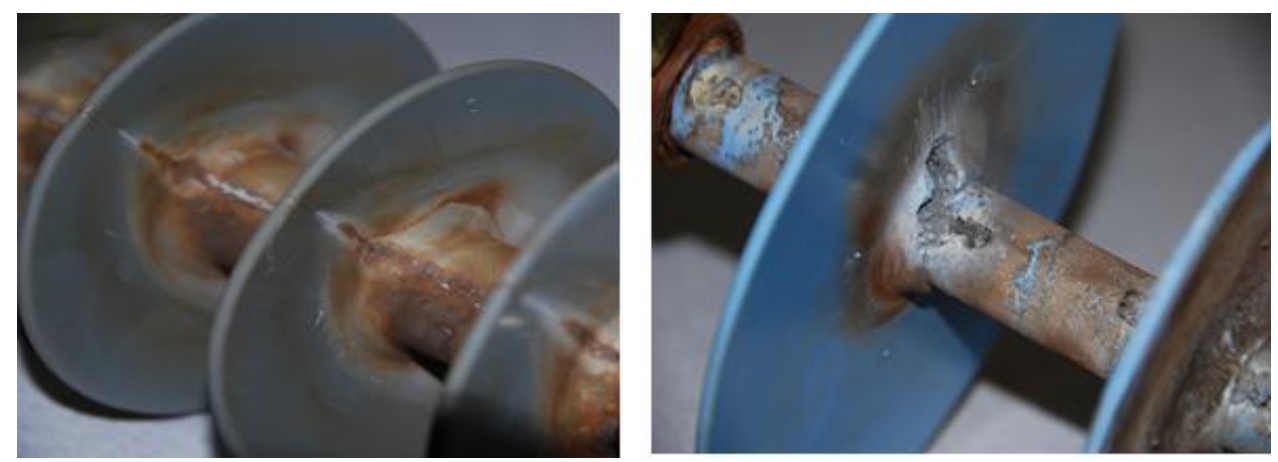

Figure 4.31: Comparison of aging of HTV SR insulators from manufacture A and manufacturer B for HVAC excitation

Figure 4.32 show the HTV SR insulator with a unified Specific Creepage Distance (USCD) of $35 \mathrm{~mm} / \mathrm{kV}$ (Channel 4) before and after the test, while Figure 4.33 shows the aging in more detail. This insulator did not age much and only shows a discoloration on the rods and sheds and light tracking on the pollution layer.
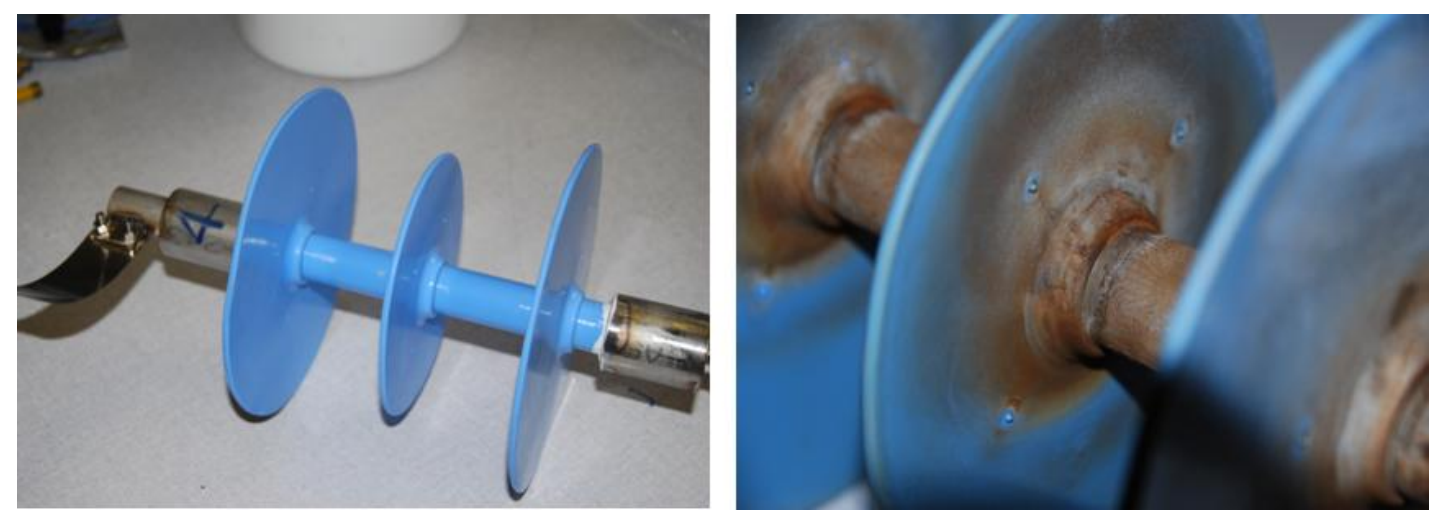

Figure 4.32: HTV SR insulator (Channel 4) before and after the test for HVAC excitation 


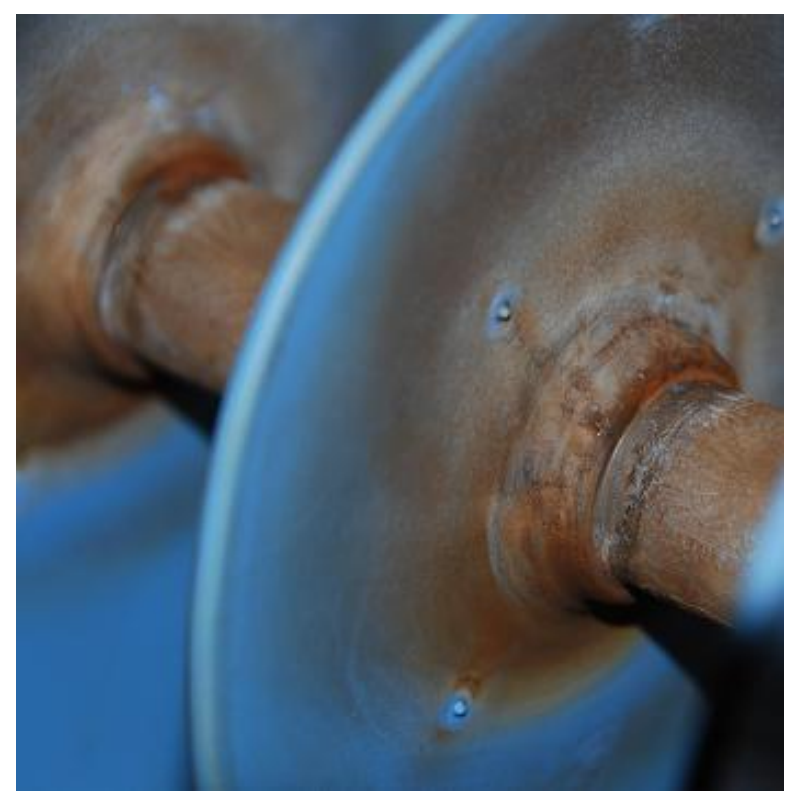

Figure 4.33: Detailed view of aging of the HTV SR insulator (Channel 4) for HVAC excitation

Figure 4.34 shows the HTV SR insulator with unified SCD of $28 \mathrm{~mm} / \mathrm{kV}$ and 35 $\mathrm{mm} / \mathrm{kV}$. The HTV SR insulator with unified SCD of $28 \mathrm{~mm} / \mathrm{kV}$ (Channel 3) has eight punctures, as well as small tracking on the pollution layer. The HTV SR insulator with unified SCD of $35 \mathrm{~mm} / \mathrm{kV}$ (Channel 4) only has slight tracks on the pollution layer and a brown discoloration. The insulator with unified SCD of 28 $\mathrm{mm} / \mathrm{kV}$ (Channel 3) performed worse compared to the insulator with unified SCD of $35 \mathrm{~mm} / \mathrm{kV}$ (Channel 4$)$ in terms of material aging.
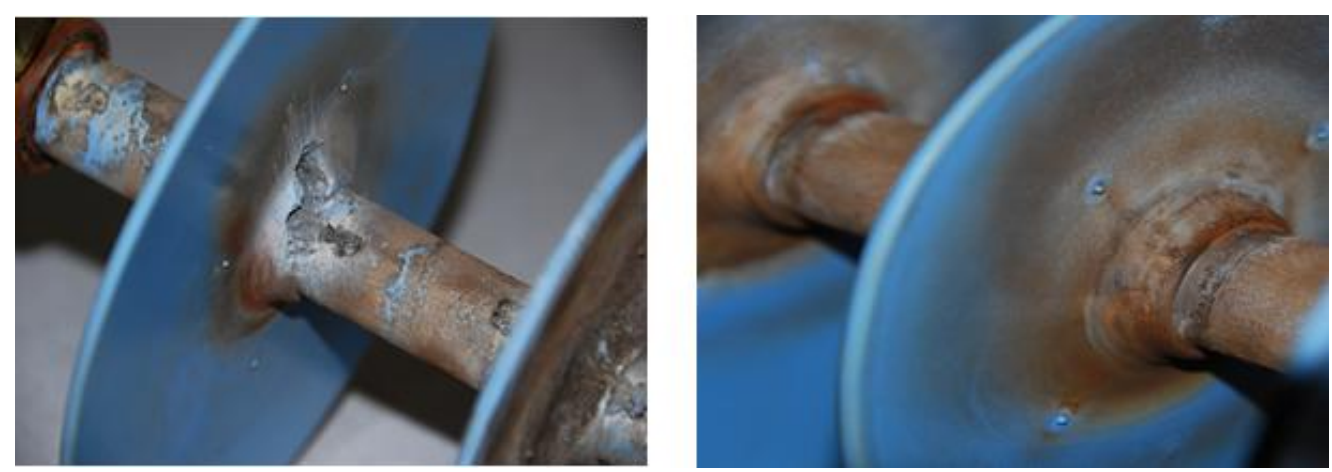

Figure 4.34: Comparison of aging of HTV SR insulators with creepage distances of $380 \mathrm{~mm}$ and $476 \mathrm{~mm}$ for HVAC excitation

Figure 4.35 show the uncoated porcelain insulator (Channel 5) before and after the test, while Figure 4.36 shows the aging in more detail. This reference porcelain insulator did not age much with time and only experienced light brown discoloration at the end of the test. The discoloration is visible when dry, though hard to see when the insulator is wet. 

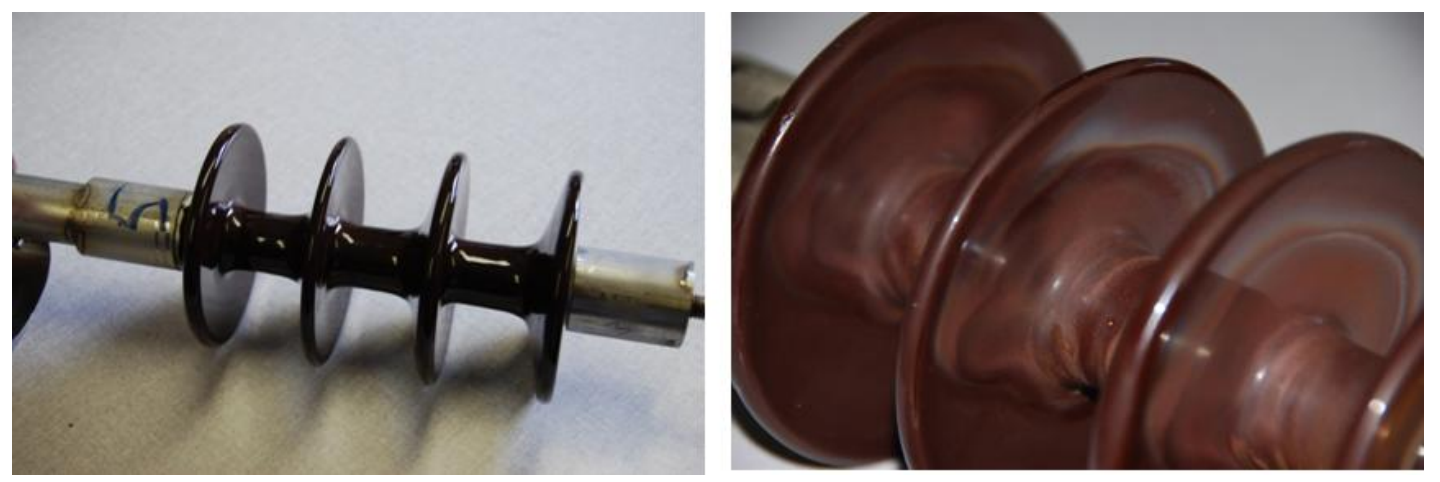

Figure 4.35: Reference porcelain insulator (Channel 5) before and after the test for HVAC excitation

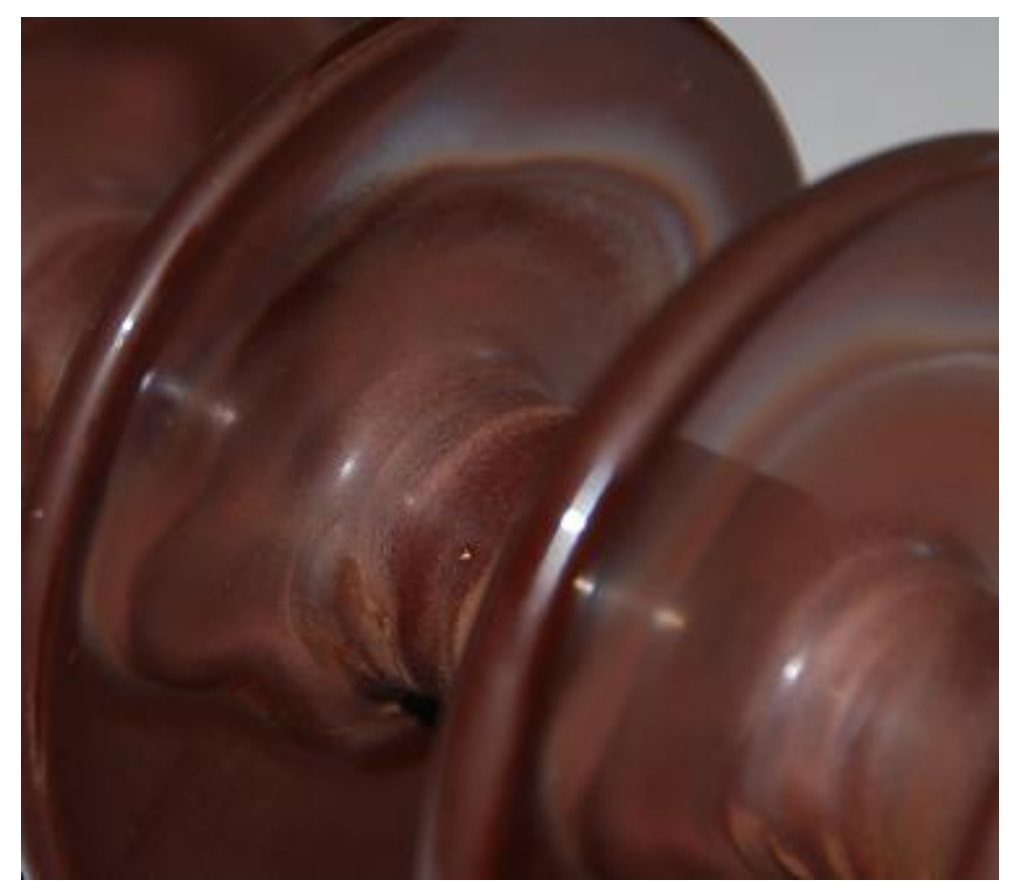

Figure 4.36: Detailed view of aging of the reference porcelain insulator (Channel 5) for HVAC excitation

Figure 4.37 show the RTV SR coated porcelain insulator (Channel 6) before and after the test, while Figure 4.38 shows the aging in more detail. This insulator developed a brown, almost reddish discoloration and exhibits severe tracks and erosion. Some of the tracks have a whitish / pinkish colour. There is severe erosion on the sheds as well as on the rods and the insulator had lost almost the entire coating on the rods and part of the sheds. There is also severe blackening of the tip of the metal end fitting. 

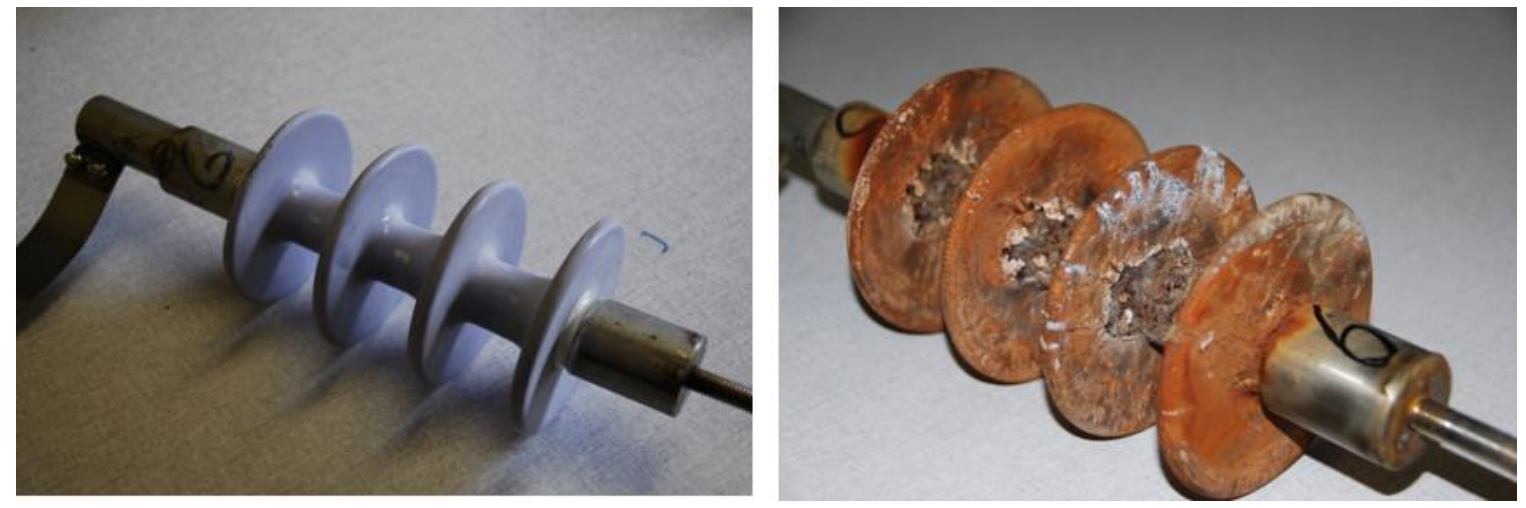

Figure 4.37: RTV SR coated porcelain insulator (Channel 6) before and after the test for HVAC excitation

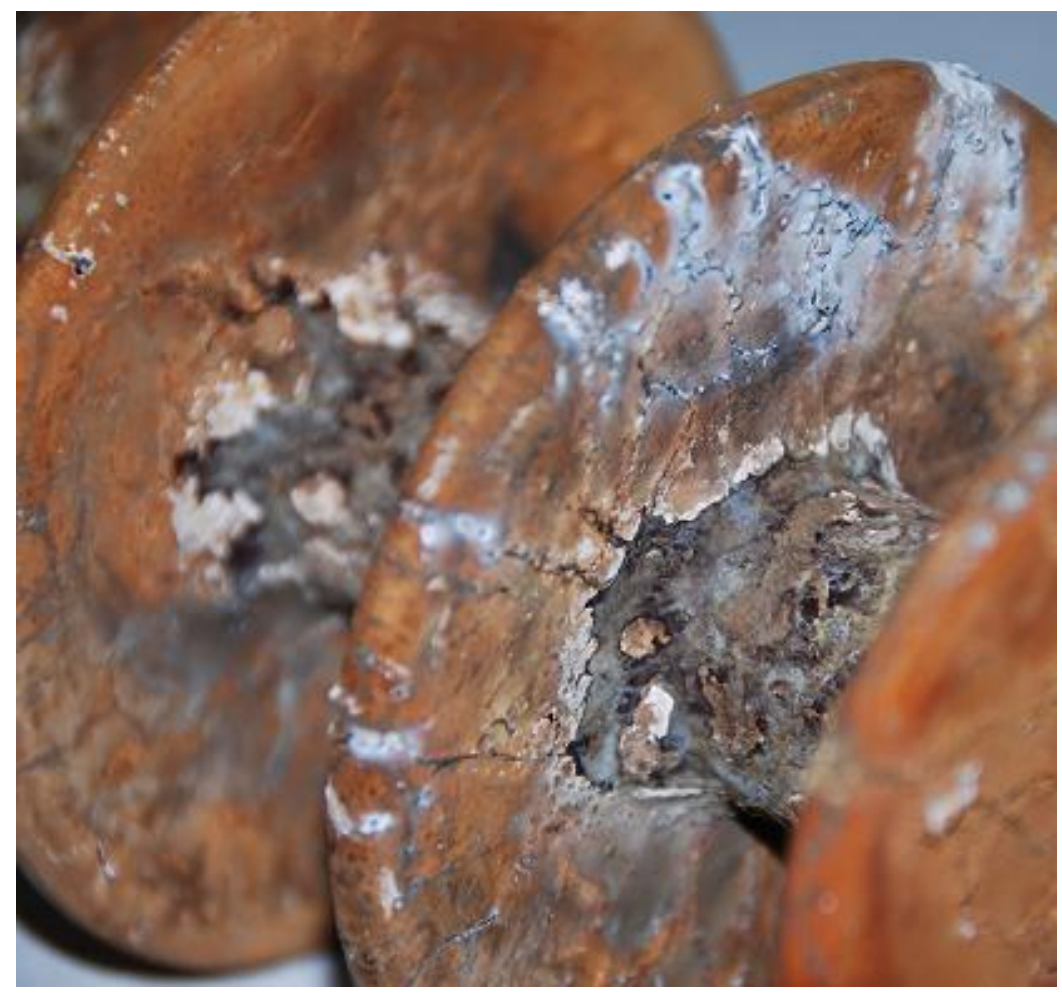

Figure 4.38: Detailed view of aging of the RTV SR coated porcelain (Channel 6) insulator for HVAC excitation

Figure 4.39 shows the porcelain (Channel 5) and RTV SR coated porcelain (Channel 6) insulators. The porcelain insulator only had minor discoloration at the end of the test, whereas the RTV SR coated porcelain had severe tracks, erosion and discoloration. This section shows that the RTV SR coated porcelain insulator (Channel 6) aged faster than the porcelain insulator (Channel 5). 

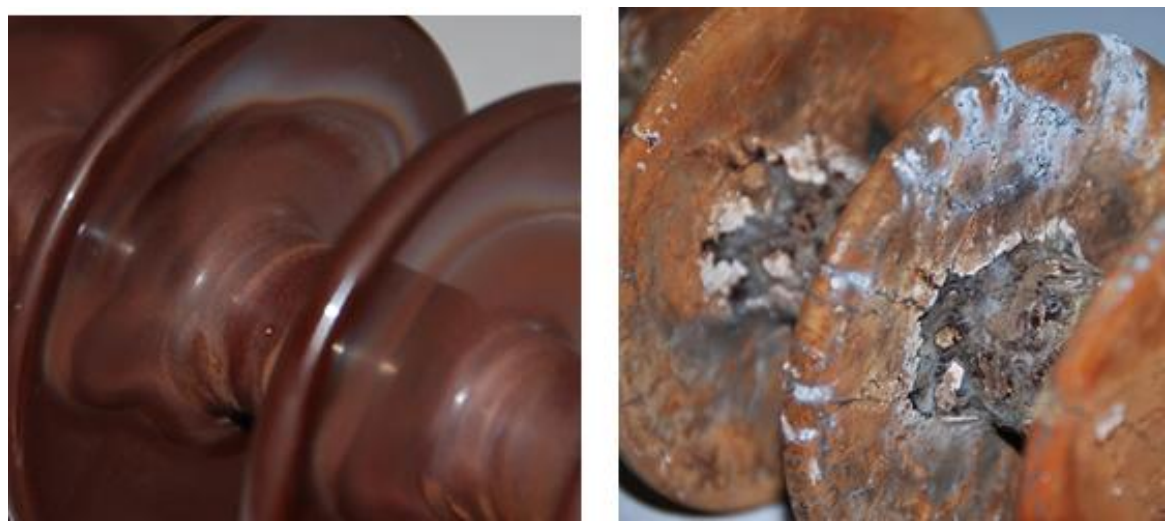

Figure 4.39: Comparison of aging of the porcelain and RTV SR coated porcelain insulators for HVAC excitation

\subsubsection{Hydrophobic properties}

Figure 4.40 compares the hydrophobicity of the EPDM (Channel 1) and HTV SR (Channel 2) insulators with a unified SCD of $28 \mathrm{~mm} / \mathrm{kV}$. The EPDM insulator (Channel 1) has a wettability class of 5, which is almost hydrophilic. The HTV SR insulator (Channel 2) has a wettability class of 1 , which is still completely hydrophobic, or has recovered its hydrophobicity. Thus, the HTV SR (Channel 2) insulator still retains its hydrophobicity, while the EPDM insulator (Channel 1) is hydrophilic.
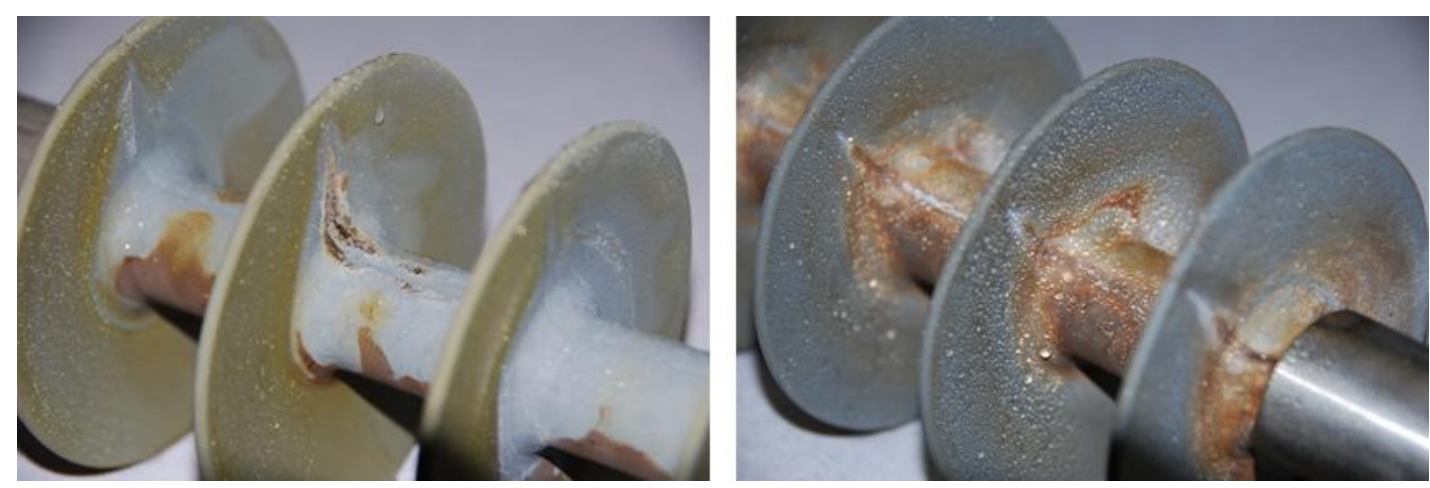

Figure 4.40: Wettability of the EPDM insulator (Channel 1) and the HTV SR insulator (Channel 2), both with a unified SCD of $28 \mathrm{~mm} / \mathrm{kV}$

Figure 4.41 compares the hydrophobicity of the HTV SR insulator with a unified SCDof $28 \mathrm{~mm} / \mathrm{kV}$ (Channel 3) and the HTV SR insulator with a unified SCD of 35 $\mathrm{mm} / \mathrm{kV}$ (Channel 4). Both insulators are still hydrophobic. The HTV SR insulator with a unified SCDof $28 \mathrm{~mm} / \mathrm{kV}$ (Channel 3) has a wettability class of either 3 or 4 , and is still hydrophobic on the sheds, but is becoming hydrophilic on the rod, especially close to where the puncture occurred. The HTV SR insulator (Channel 4) with a unified SCD of $35 \mathrm{~mm} / \mathrm{kV}$, has a wettability class of 1 , and is still completely 
hydrophobic. Thus, in comparison, the insulator with a longer unified SCD retains its hydrophobicity better.
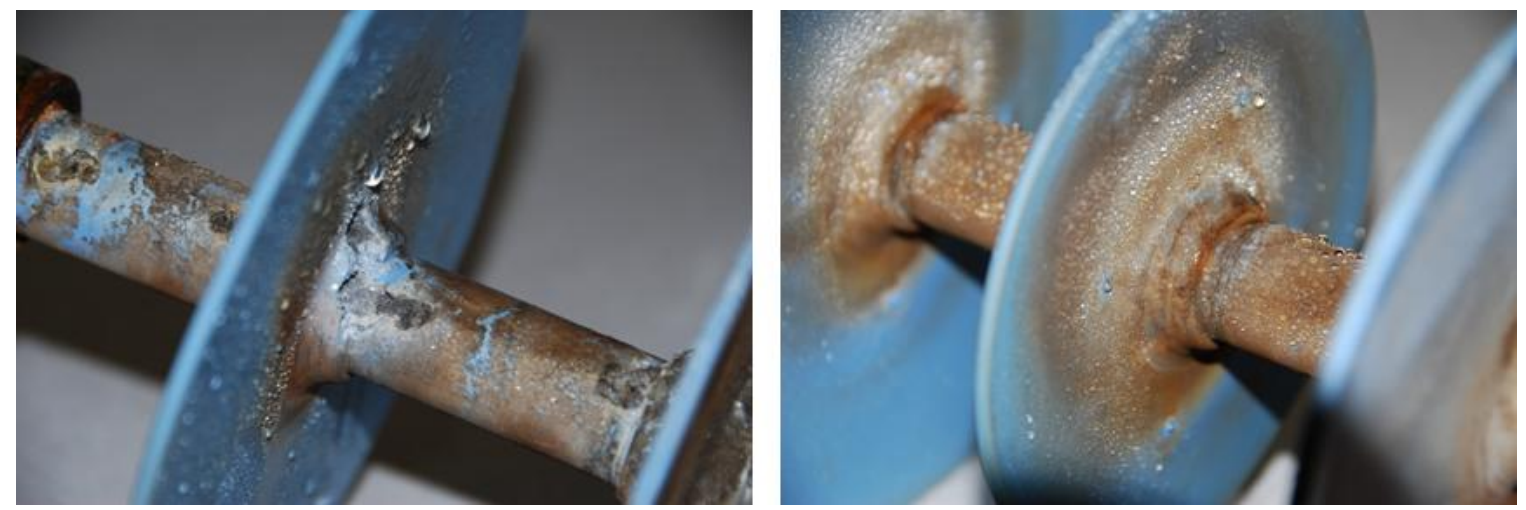

Figure 4.41: Wettability of the HTV SR insulator with a unified SCDof $28 \mathrm{~mm} / \mathrm{kV}$ (Channel 3) and the HTV SR insulator with a unified SCD of $35 \mathrm{~mm} / \mathrm{kV}$ (Channel 4)

Figure 4.42 compares the hydrophobicity of the reference porcelain insulator (Channel 5) and the RTV SR coated porcelain insulator (Channel 6). The reference uncoated porcelain insulator (Channel 5) has a wettability class of 6, and is completely hydrophilic. The RTV SR coated porcelain insulator has a wettability class of 4 , and is almost becoming hydrophilic. In comparison, it follows that coating improves the hydrophobicity of the insulator.
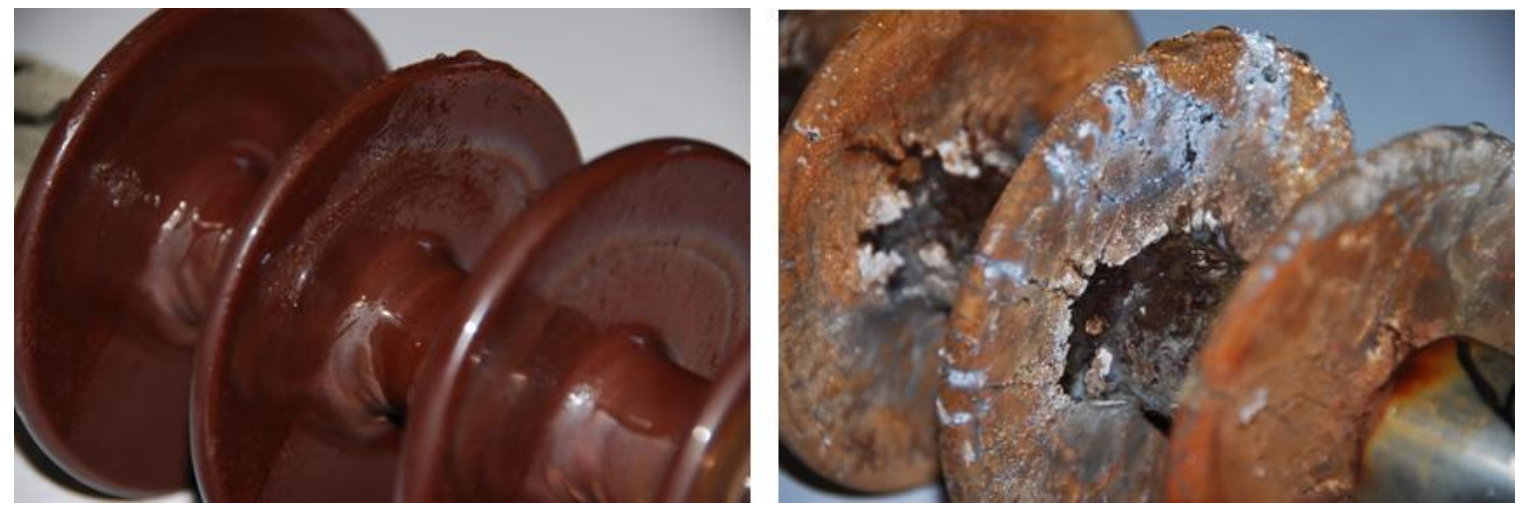

Figure 4.42: Wettability of the reference porcelain insulator (Channel 5) and the RTV SR coated porcelain insulator (Channel 6)

\subsection{Second test series: Positive polarity HVDC excitation}

\subsubsection{Overview}

The second series of test was repeated with for the six insulator samples shown in Table 4.4, using positive polarity HVDC excitation. This experiment was also based on a combination of the IEC 61302 and the IEC 62217 (Annex A) standards. The 
experiment was started on the $15^{\text {th }}$ July 2008 at around 09:47, and it was stopped on the $1^{\text {st }}$ September 2008.

\subsubsection{Test methodology and test procedures}

Stainless-steel metal end fittings were used for the end parts of the insulators. The tests were conducted with an applied voltage of $13.6 \mathrm{kV}$ and salt-solution conductivity of $0.243[\mathrm{~S} / \mathrm{m}] @ 20^{\circ} \mathrm{C}$ as in the second series of tests with HVAC excitation.

This system was monitored on a daily basis with the view to record visual observations of the discharges or any other important changes. Pictures and video recordings were recorded on a weekly basis. The following procedure was used at the beginning of the experiment and for restarting the experiment after an interruption:

- $\quad$ Switch on the Tracking Wheel Tester (TWT) drive motor.

- Lock the door to the test chamber and remove the earth stick attached to the transformer.

- Reset the emergency stop button.

- $\quad$ Switch on the main circuit breaker (400 A).

- $\quad$ Set the variac is set to zero.

- Switch on the variac's main contactor.

- $\quad$ Reset the protection circuit.

- $\quad$ Adjust the variac until a voltage of $13.6 \mathrm{kV}$ is displayed on the $\mathrm{HV}$ side of the transformer.

In order to make sure that repeatable results are obtained and that the tests conform to the relevant test standard, the test arrangement required close supervision. The following operating procedures were implemented to this effect:

- Maintain the water level within $\pm 10 \mathrm{~mm}$ of nominal.

- Maintain the conductivity of the liquid at $2.43[\mathrm{mS} / \mathrm{cm}]$ at $20^{\circ} \mathrm{C}$.

- $\quad$ Maintain the temperature of the liquid at $(25 \pm 5)^{\circ} \mathrm{C}$.

- Examine the wheel every 24 hours to make sure that hardware is working properly. 
The following operating procedure was followed in case of a fault:

- $\quad$ Press the emergency stop button to disconnect the LV supply.

- $\quad$ Switch off the motor.

- $\quad$ Open the chamber door and apply the earth stick to the HV tap.

- $\quad$ Remove the failed specimen and replace with a new specimen.

- Restart the process.

The following operating procedure was followed to stop the machine:

- $\quad$ Set the Variac to zero.

- Switch off the Variac's main contactor.

- $\quad$ Unlock the door and apply the earth stick to the HV Supply.

\subsubsection{Summary of test results}

\subsubsection{Leakage current}

Table 4.8 and Figure 4.43 summarize the leakage current results for the EPDM (Channel 1) and HTV SR (Channel 2) insulators. Both these insulators showed dry band arcs at the start of the test, while the other insulators started experiencing dry band arcs only after a couple of hours or even days later. At the beginning of the experiment the EPDM insulator (Channel 1) had a peak current of $40 \mathrm{~mA}$ while the HTV SR insulator (Channel 2) had a peak current of $30 \mathrm{~mA}$. After approximately three days, the HTV SR insulator (Channel 2) lost its hydrophobicity and a rise in peak current was experienced. However, on the $11^{\text {th }}$ August 2008, the HTV SR insulator (Channel 3) tripped and thus caused the whole system to malfunction. The system could only be started again on the $19^{\text {th }}$ August 2008 . At the end of the experiment the EPDM insulator (Channel 1) had a peak current of approximately 55 mA while HTV SR insulator (Channel 2) had a peak current of $151 \mathrm{~mA}$. This shows that the EPDM insulator performed better than the HTV SR insulator in the sense that it had lower peak currents compared to the HTV SR insulator. However, this condition only applied after the hydrophobicity of the HTV SR insulator was lost. 
Table 4.8: Peak leakage currents for Channel 1 and Channel 2 for positive polarity HVDC excitation

\begin{tabular}{|l|l|l|}
\hline & Channel 1 (EPDM) & Channel 2 (HTV SR) \\
\hline $\begin{array}{l}\text { Peak current [mA] in the } \\
\text { beginning }\end{array}$ & $30 \mathrm{~mA}$ \\
\hline Hydrophobicity lost & $\begin{array}{l}\text { Hydrophobicity } \\
\text { immediately after voltage is } \\
\text { applied }\end{array}$ & $\begin{array}{l}\text { Hydrophobicity lost } \\
\text { immediately after voltage is } \\
\text { applied }\end{array}$ \\
\hline Peak current [mA] in the end & $55 \mathrm{~mA}$ & $151 \mathrm{~mA}$ \\
\hline
\end{tabular}

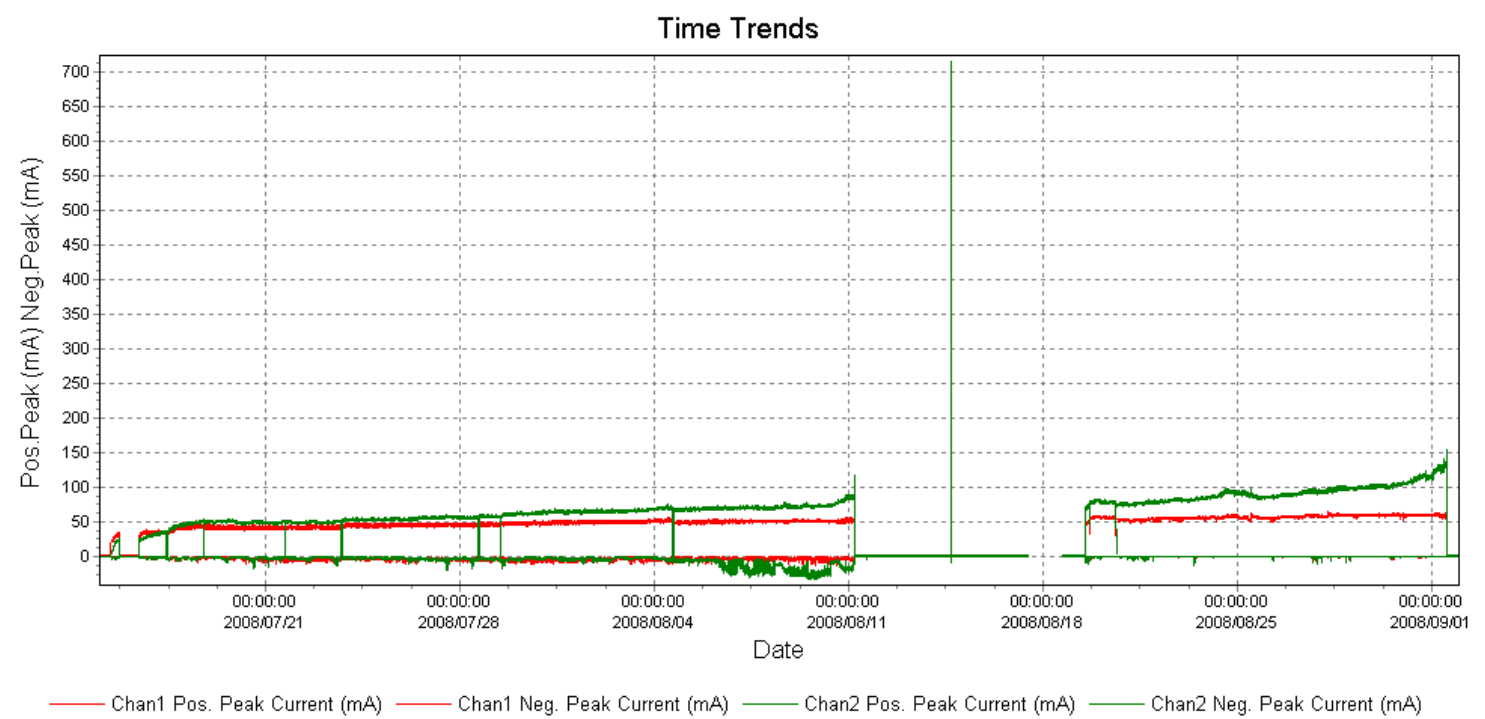

Figure 4.43: Peak leakage currents for the EPDM (Channel 1) and HTV SR (Channel 2) insulators for positive polarity HVDC excitation.

Table 4.9 and Figure 4.44 summarize the leakage current results for the HTV SR (Channel 2) and HTV SR (Channel 3) insulators. The insulators have the same unified SCD of $28 \mathrm{~mm} / \mathrm{kV}$ but different profiles and at the beginning of the experiment the HTV SR insulator from manufacturer A (Channel 2) and the HTV SR insulator from manufacturer B (Channel 3) both had a peak leakage current of approximately $30 \mathrm{~mA}$. However, the trend for the next three weeks shows that HTV SR insulator from manufacturer A (Channel 2) had a slightly higher peak current than HTV SR insulator from manufacturer B (Channel 3). Channel 3 then experienced its first puncture, which caused a sudden rise in the peak current, until the insulator eventually tripped on the $11^{\text {th }}$ August 2008 at a peak current of $724 \mathrm{~mA}$. This insulator was removed and the test restarted. At the end of the experiment the HTV SR insulator from manufacturer A (Channel 2) had a positive peak current of $151 \mathrm{~mA}$. Thus, the HTV SR insulator from manufacturer A (Channel 2) performed better compared to the HTV SR insulator from manufacturer B (Channel 3). 
Table 4.9: Peak leakage currents for Channel 1 and Channel 2 for positive polarity HVDC excitation

\begin{tabular}{|l|l|l|}
\hline & $\begin{array}{l}\text { Channel 2 (HTV SR insulator } \\
\text { from manufacturer A) }\end{array}$ & $\begin{array}{l}\text { Channel 3 (HTV SR insulator } \\
\text { from manufacturer A) }\end{array}$ \\
\hline $\begin{array}{l}\text { Peak current }[\mathrm{mA}] \text { in the } \\
\text { beginning }\end{array}$ & $30 \mathrm{~mA}$ & $30 \mathrm{~mA}$ \\
\hline Peak current $[\mathrm{mA}]$ in the end & $151 \mathrm{~mA}$ & $\begin{array}{l}\text { "Puncture" } \\
724 \mathrm{~mA}\end{array}$ \\
\hline
\end{tabular}

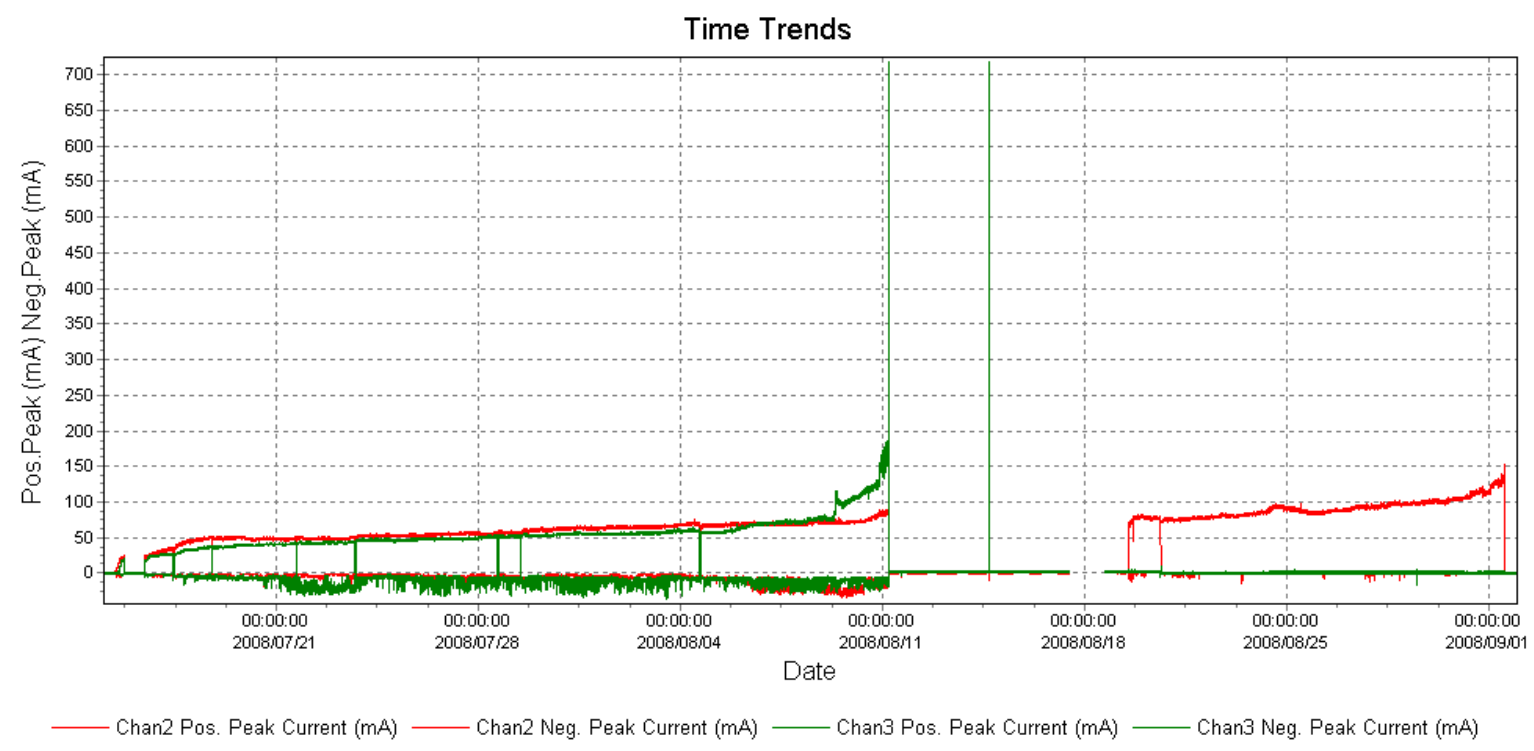

Figure 4.44: Peak leakage currents for HTV SR insulators from manufacturer A (Channel 2) and manufacturer B (Channel 3) for positive polarity HVDC excitation

Table 4.10 and Figure 4.45 summarize the leakage current results for the HTV SR insulator with a unified SCD of $28 \mathrm{~mm} / \mathrm{kV}$ (Channel 3) and the HTV SR insulator with a unified SCD of $35 \mathrm{~mm} / \mathrm{kV}$ (Channel 4). When the experiment started on the $15^{\text {th }}$ July 2008, only the insulators belonging to Channel 1 and Channel 2 had dry band arcs. However, after about $2 \mathrm{~h} 30$ minutes, the insulators belonging to Channel 3 and Channel 4 began to experience dry band arcs. At the beginning of the experiment the HTV SR insulator with a unified SCD of $28 \mathrm{~mm} / \mathrm{kV}$ (Channel 3) had a peak current of $30 \mathrm{~mA}$, while HTV SR insulator with a unified SCD of $35 \mathrm{~mm} / \mathrm{kV}$ (Channel 4) had a peak current of $20 \mathrm{~mA}$. Channel 3 experienced a rise in peak current until it tripped at $724 \mathrm{~mA}$ on the $11^{\text {th }}$ August 2008 and was removed from the wheel. After restart, Channel 4 also experienced a rise in peak currents until it tripped on the $1^{\text {st }}$ September 2008 at a peak current of approximately $420 \mathrm{~mA}$. Therefore, it is concluded that the insulator with the shortest unified SCD (Channel 3) performed worse than the insulator with the longer unified SCD (Channel 4) in terms of leakage current. 
Table 4.10: Peak leakage currents for Channel 3 and Channel 4 for positive polarity HVDC excitation

\begin{tabular}{|l|l|l|}
\hline & $\begin{array}{l}\text { Channel 3 (Unified SCDof 28 } \\
\mathbf{m m} / \mathbf{k V} \text { ) }\end{array}$ & $\begin{array}{l}\text { Channel 4 (Unified SCDof 35 } \\
\mathbf{m m} / \mathbf{k V} \text { ) }\end{array}$ \\
\hline $\begin{array}{l}\text { Peak current [mA] in the } \\
\text { beginning }\end{array}$ & $30 \mathrm{~mA}$ & $20 \mathrm{~mA}$ \\
\hline Hydrophobicity lost & $\begin{array}{l}\text { Hydrophobicity lost 2h30 } \\
\text { minutes after test is started }\end{array}$ & $\begin{array}{l}\text { Hydrophobicity lost 2h30 } \\
\text { minutes after test is started }\end{array}$ \\
\hline Peak current [mA] in the end & $\begin{array}{l}724 \mathrm{~mA} \\
\text { "Puncture" }\end{array}$ & $95 \mathrm{~mA}$ \\
\hline
\end{tabular}

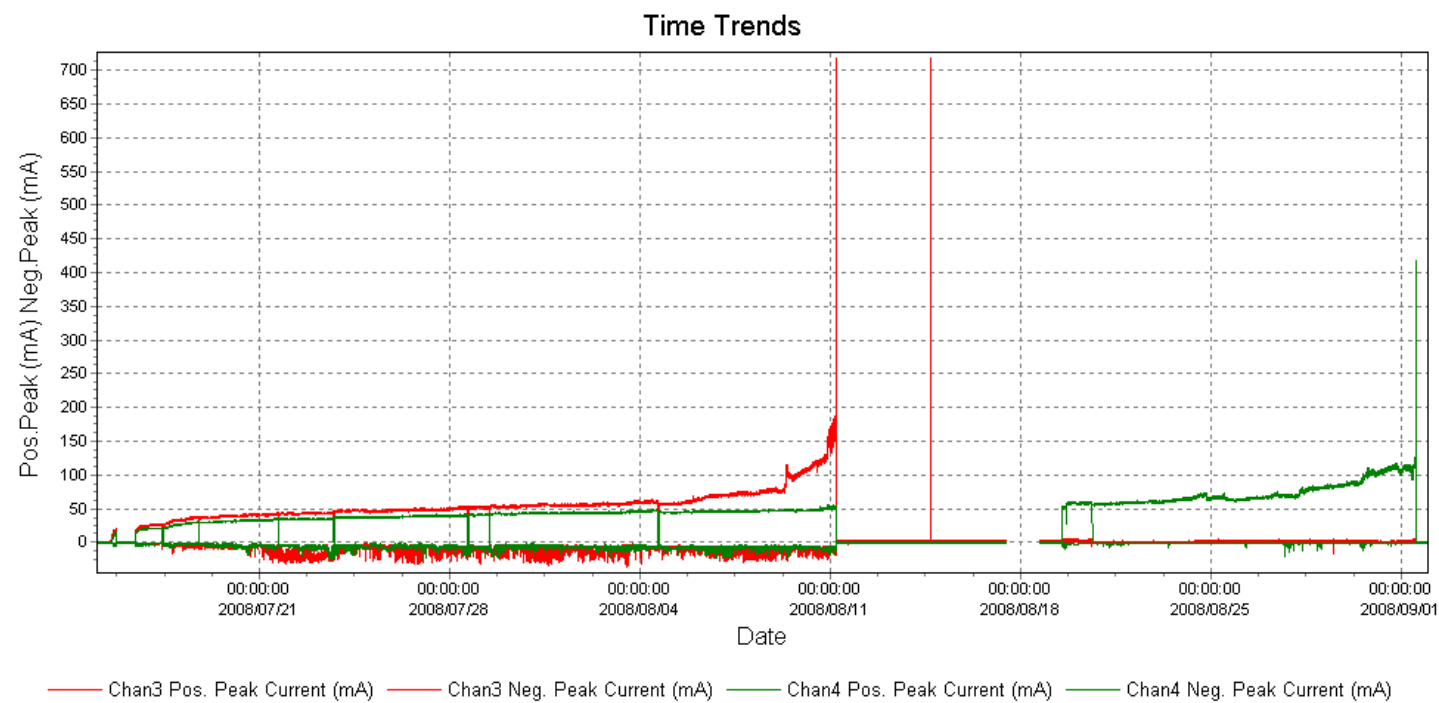

Figure 4.45: Peak leakage currents for the HTV SR insulator with SCD of $28 \mathrm{~mm} / \mathrm{kV}$ (Channel 3) and the HTV SR insulator with SCD of $35 \mathrm{~mm} / \mathrm{kV}$ (Channel 4) forh positive polarity HVDC excitation

Table 4.11 and Figure 4.46 summarize the leakage current results for the porcelain insulator (Channel 5) and the RTV SR coated porcelain insulator (Channel 6). The insulator that belongs to Channel 5 (reference porcelain) started experiencing dry band arcs $2 \mathrm{~h} 30$ minutes after commencement of the experiment, while insulator that belongs to Channel 6 (RTV SR coated porcelain) only started showing signs of arcing on the $3^{\text {rd }}$ day of the experiment. At the beginning of the experiment the reference porcelain insulator (Channel 5) had a peak current of $40 \mathrm{~mA}$, while the RTV SR coated porcelain insulator (Channel 6) had a peak current of $55 \mathrm{~mA}$. Channel 6 experienced a rise in peak current until it eventually tripped on the $20^{\text {th }}$ August 2008 at $724 \mathrm{~mA}$. At the end of the experiment Channel 5 had a peak current of $95 \mathrm{~mA}$. Therefore, the coating on the insulator improved its performance in the beginning. After the hydrophobicity was lost, however, the coated insulator (Channel 6) performed worse than the uncoated one (Channel 5). 
Table 4.11: Peak leakage currents for Channel 5 and Channel 6 for positive polarity HVDC excitation

\begin{tabular}{|l|l|l|}
\hline & Channel 5 (Porcelain) & $\begin{array}{l}\text { Channel 6 (RTV SR coated } \\
\text { porcelain) }\end{array}$ \\
\hline $\begin{array}{l}\text { Peak current [mA] in the } \\
\text { beginning }\end{array}$ & $40 \mathrm{~mA} \mathrm{~mA}$ \\
\hline Hydrophobicity lost & $\begin{array}{l}\text { Hydrophobicity lost 2h30 } \\
\text { minutes after commencement of } \\
\text { tests. }\end{array}$ & $\begin{array}{l}\text { Hydrophobicity lost 3 days after } \\
\text { voltage is applied. }\end{array}$ \\
\hline Peak current [mA] in the end & $95 \mathrm{~mA}$ & $\begin{array}{l}\text { "Puncture" } \\
724 \mathrm{~mA}\end{array}$ \\
\hline
\end{tabular}

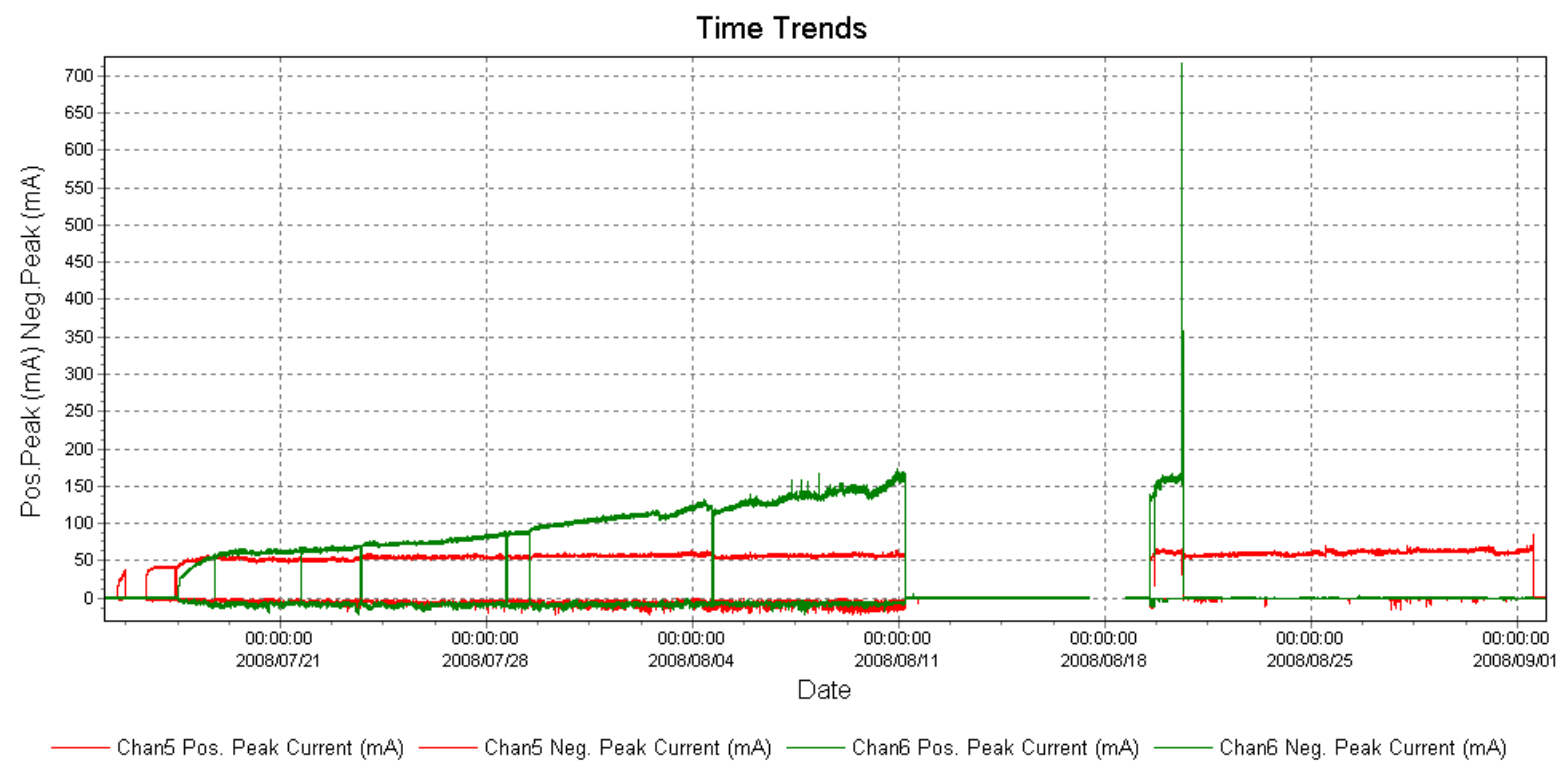

Figure 4.46: Peak leakage currents for Channel 5 (porcelain) and Channel 6 (RTV SR coated porcelain) for positive polarity HVDC excitation

\subsubsection{Visual observations of aging}

Visual observations were made of the test insulators on a daily basis with the view to determine how the insulators aged with time. Photographs and video recordings of the discharges of the insulators were taken on a weekly basis.

Figure 4.47 shows the EPDM insulator with a unified SCD of $28 \mathrm{~mm} / \mathrm{kV}$ (Channel 1) before and after the test, while Figure 4.48 shows the aging in more detail. It shows discoloration on the insulator sheds and rods as well as on the metal end fittings. Furthermore, there is light tracking displayed on the pollution layer and heavy erosion. The insulator appears to be disfigured along the mould lines and there also appears to be small black burns on the $4^{\text {th }}$ shed. 

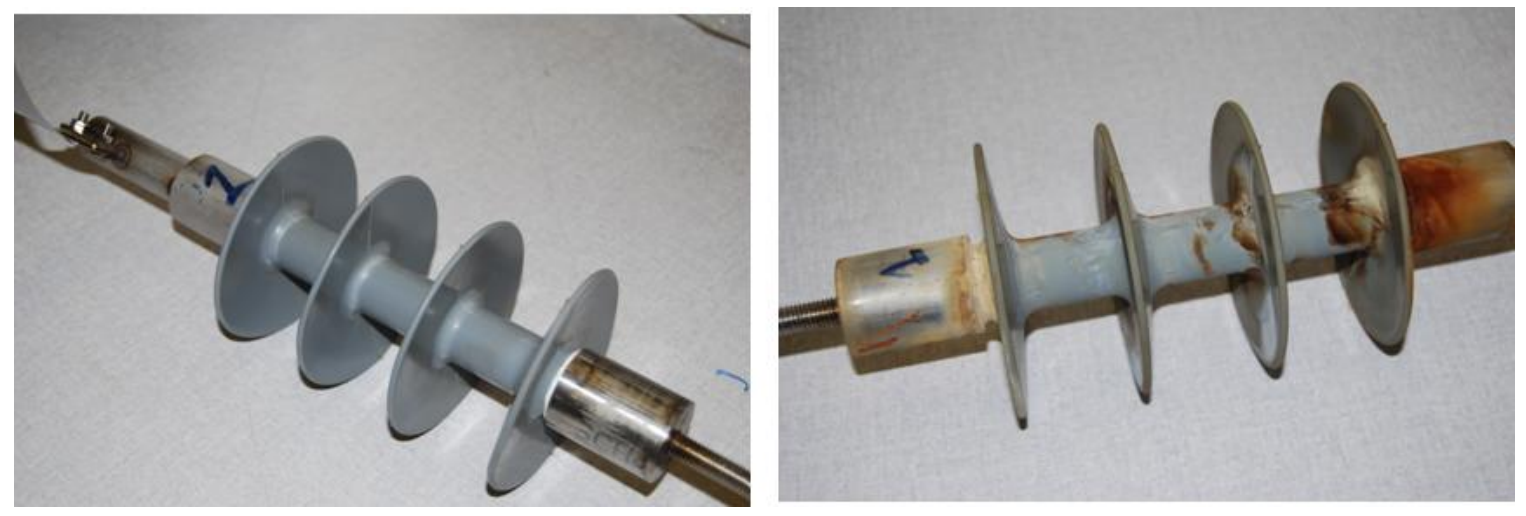

Figure 4.47: The EPDM insulator with a unified SCD of $28 \mathrm{~mm} / \mathrm{kV}$ (Channel 1) before and after the test with positive polarity HVDC excitation

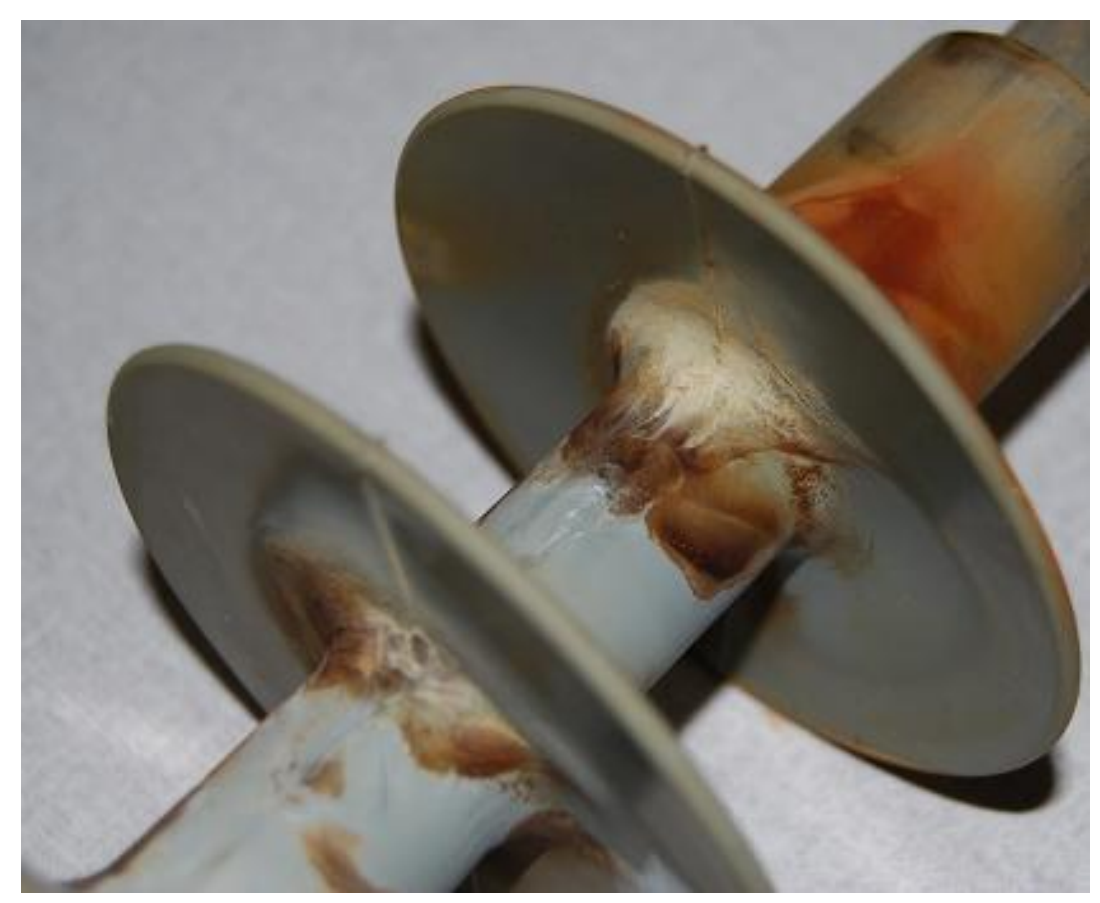

Figure 4.48: Detailed view of aging of the EPDM insulator (Channel 1) for positive polarity HVDC excitation

Figure 4.49 shows the HTV SR insulator with a unified SCD of $28 \mathrm{~mm} / \mathrm{kV}$ from manufacturer A (Channel 2) before and after the test, while Figure 4.50 shows the aging in more detail. The insulator shows severe tracking and erosion. The tracking is not only on the pollution layer, but penetrates deep into the material itself. The insulator also has one small puncture and there is severe discoloration, especially on the $4^{\text {th }}$ shed. A small part of the metal end fittings are burned. The mould line seems to have completely disappeared on one side, and only shows a little bit on the other side. 

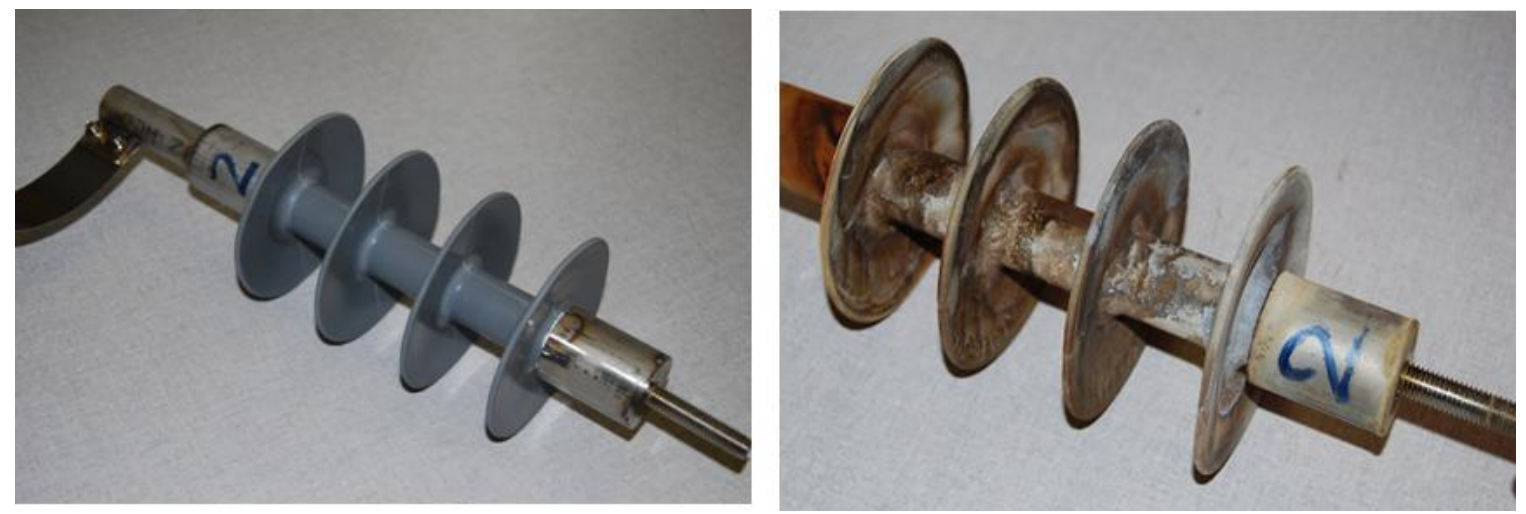

Figure 4.49: The HTV SR insulator with a unified SCD of $28 \mathrm{~mm} / \mathrm{kV}$ from manufacturer A (Channel 2) before and after the test with positive polarity HVDC excitation

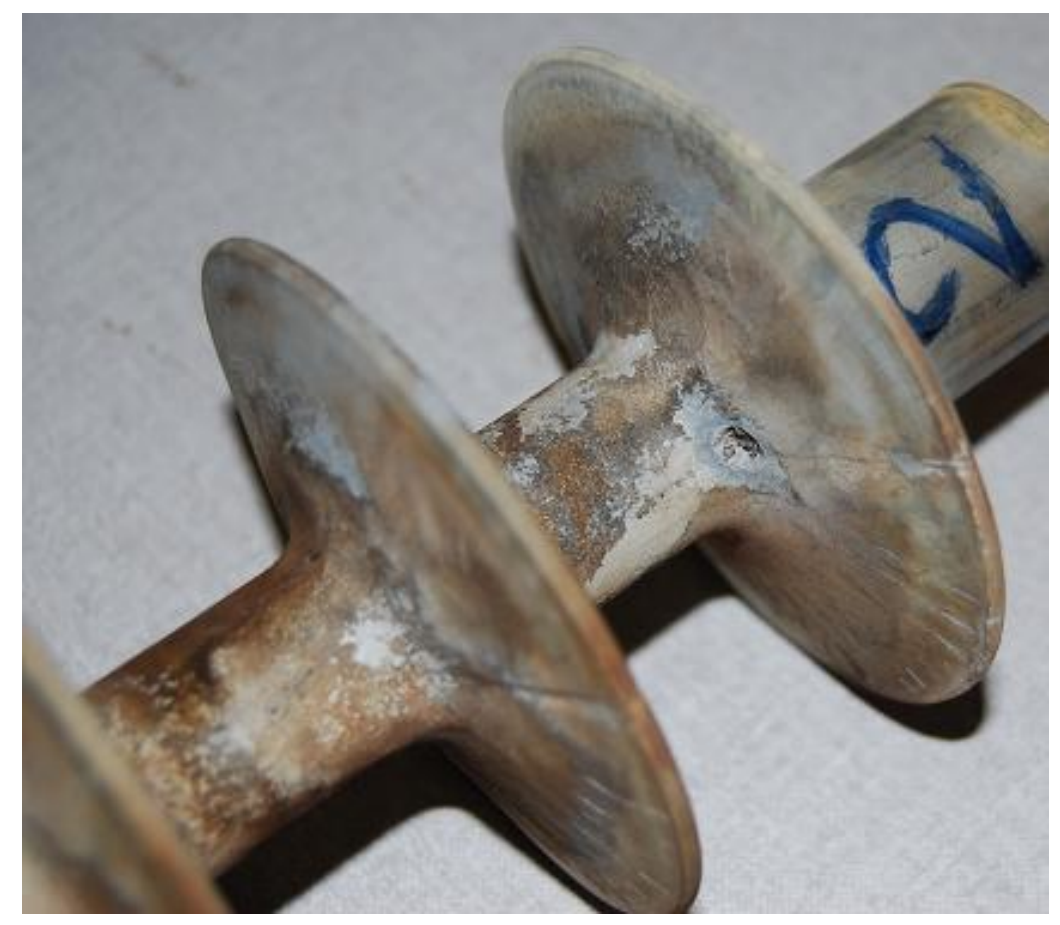

Figure 4.50: Detailed view of aging of the HTV SR insulator (Channel 2) for positive polarity HVDC excitation

Figure 4.51 shows the EPDM insulator (Channel 1) and HTV SR insulator (Channel 2) for positive polarity HVDC excitation. The EPDM insulator (Channel 1) had deformation on the mould lines, while HTV SR insulator (Channel 2) didn't show any sign of deformation on the mould lines. The HTV SR insulator (Channel 2) showed heavy signs of tracking on the pollution layer and even penetrating deeper to the material, while the EPDM insulator (Channel 1) only had small tracks on the pollution layer. Both insulators showed signs of erosion. The EPDM insulator showed deep erosion and the HTV SR insulator showed flaking of the material. Both insulators experienced discoloration, as well as burning off at the tip of the metal end fitting. 
The HTV SR insulator (Channel 2) seems to have aged severely compared to EPDM insulator (Channel 1).
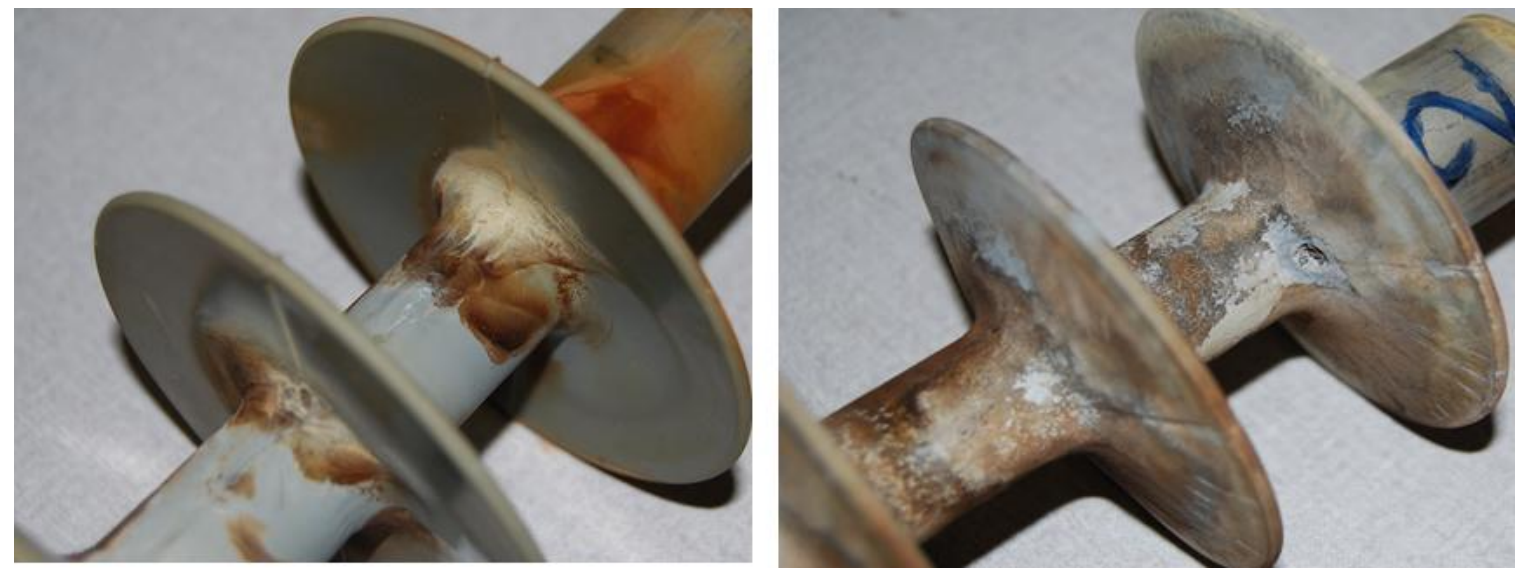

Figure 4.51: Comparison of aging of EPDM and HTV SR insulators for positive polarity HVDC excitation

Figure 4.52 shows the HTV SR insulator with a unified SCD of $28 \mathrm{~mm} / \mathrm{kV}$ from manufacturer B (Channel 3) before and after the test, while Figure 4.53 shows the aging in more detail. This insulator was so severely damaged that it caused the whole system to malfunction when it tripped. The insulating material on the rod, as well as the glass-fibre rod itself, was severely burned. Tracking appeared both on the pollution layer as well as on the material itself. The insulator also had erosion and discoloration on the sheds.
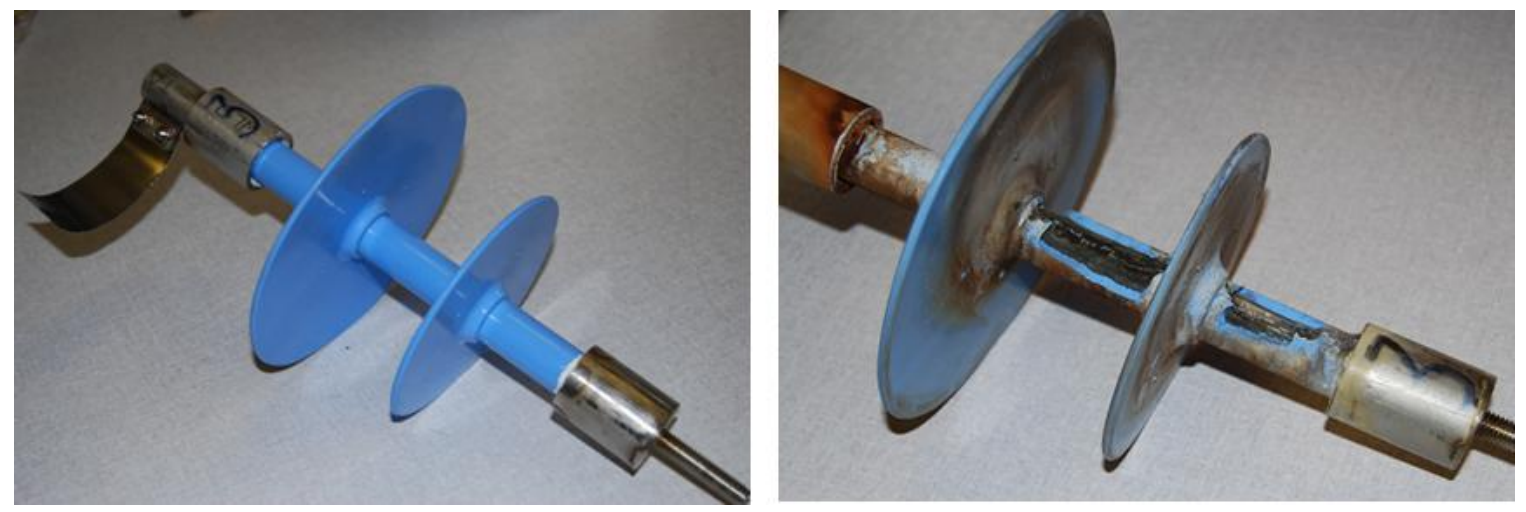

Figure 4.52: The HTV SR insulator with a unified SCD of $28 \mathrm{~mm} / \mathrm{kV}$ from manufacturer B (Channel 3) before and after the test with positive polarity HVDC excitation 


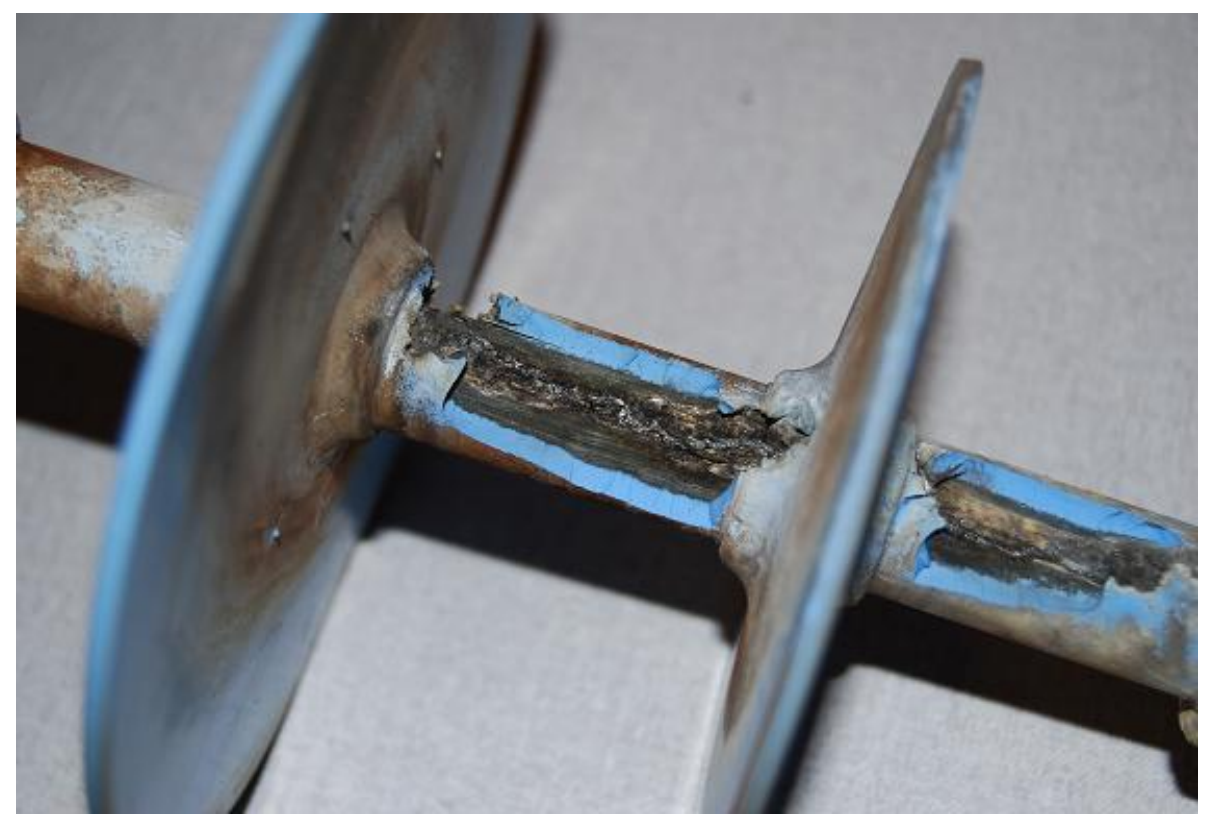

Figure 4.53: Detailed view of aging of the HTV SR insulator (Channel 3) for positive polarity HVDC excitation

Figure 4.54 shows HTV SR insulators from manufacturer A (Channel 2) and manufacturer B (Channel 3). The HTV SR insulator from manufacturer A (Channel 2) had punctures and severe erosion and tracking. It still performed better than HTV SR insulator from manufacturer B (Channel 3), which had lots of punctures until it failed, with erosion and tracking in a star pattern.
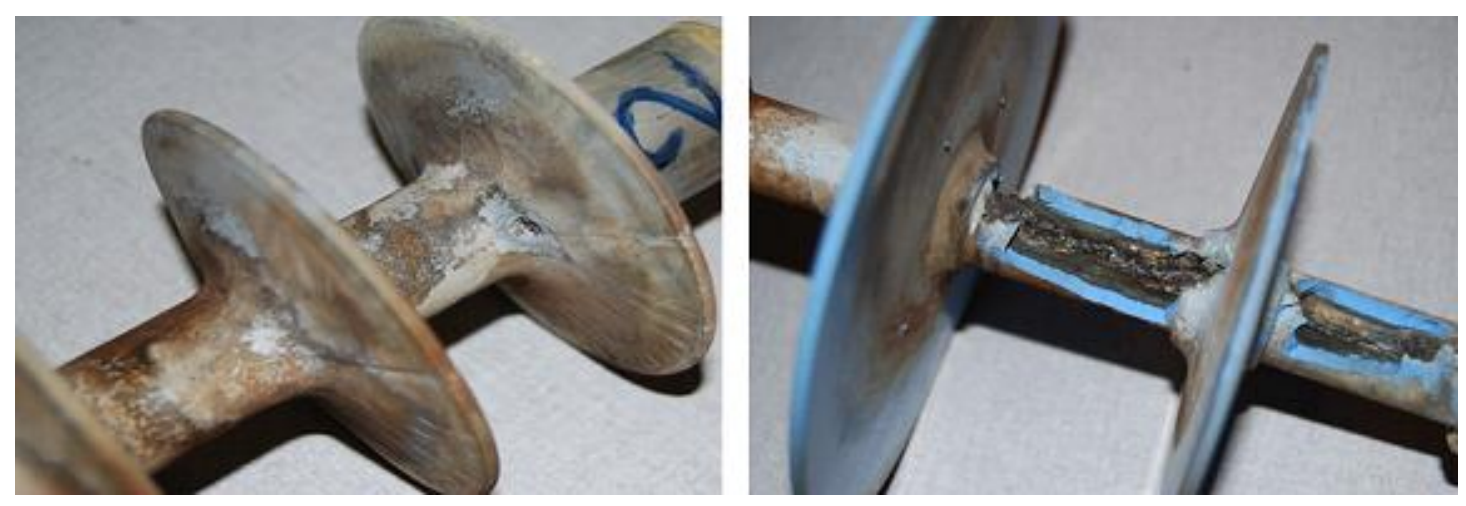

Figure 4.54: Comparison of aging of the HTV SR insulators from manufacturer A and manufacturer B for positive polarity HVDC excitation

Figure 4.55 shows the HTV SR insulator with a unified SCD of $35 \mathrm{~mm} / \mathrm{kV}$ from manufacturer B (Channel 4) before and after the test, while Figure 4.56 shows the aging in more detail. The insulator developed eight punctures and also had severe tracking and erosion in the form of a star effect. The tracking appeared only on the 
pollution layer, with whitening on the material itself. There was a severe loss of material, as well as discoloration on the insulator sample itself.
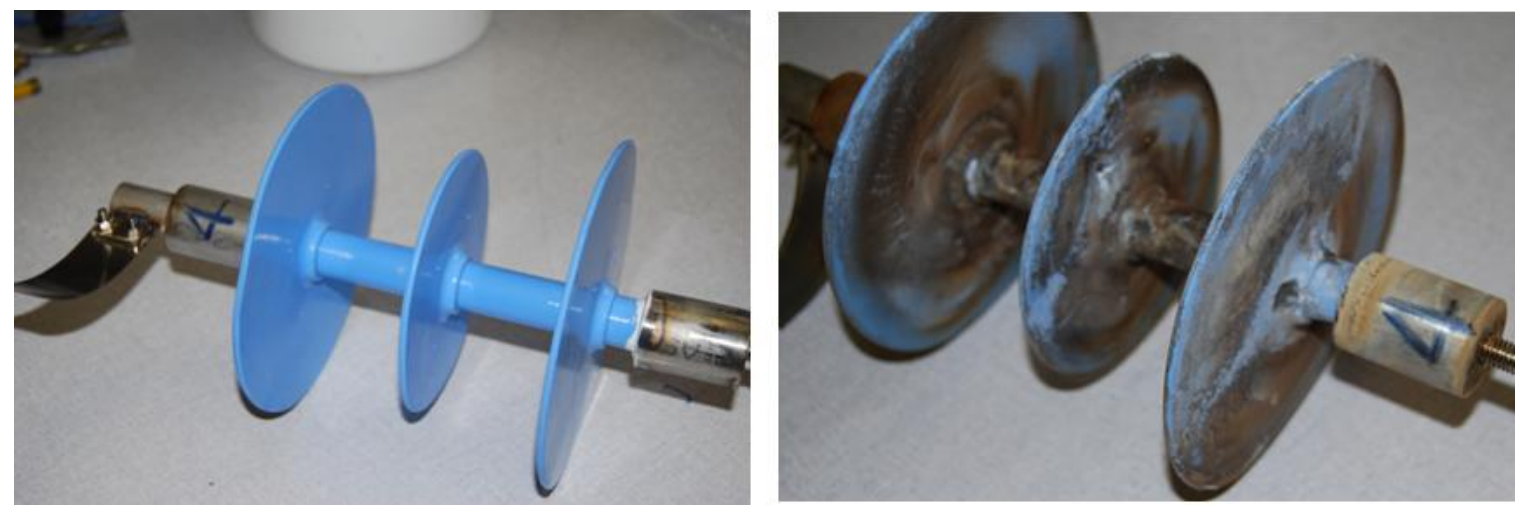

Figure 4.55: The HTV SR insulator with a unified SCD of $35 \mathrm{~mm} / \mathrm{kV}$ (Channel 4) before and after the test with positive polarity HVDC excitation

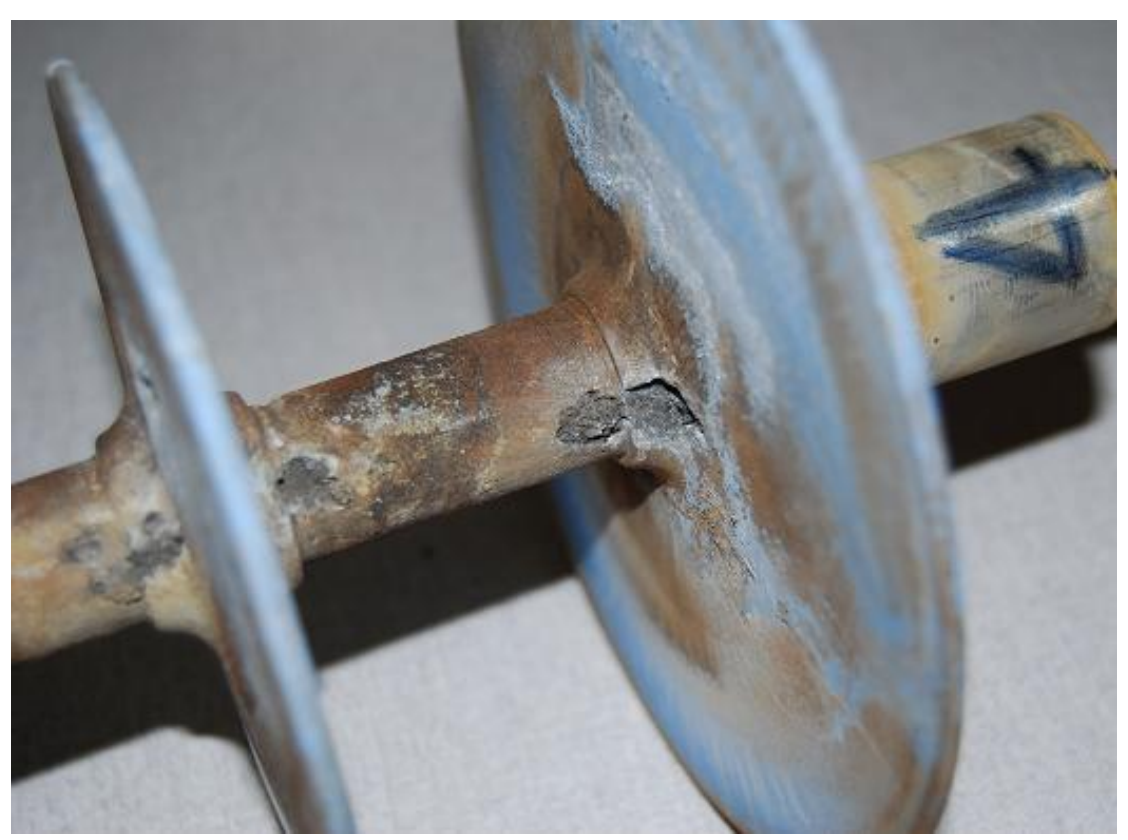

Figure 4.56: Detailed view of aging of the HTV SR insulator (Channel 4) for positive polarity HVDC excitation

Figure 4.57 shows the HTV SR insulators with unified SCDof $28 \mathrm{~mm} / \mathrm{kV}$ (Channel 3) and $35 \mathrm{~mm} / \mathrm{kV}$ (Channel 4). The insulator with the shortest unified SCD (Channel 3) had the most damage to the insulator and thus performed worse than the insulator with the longer unified SCD. 

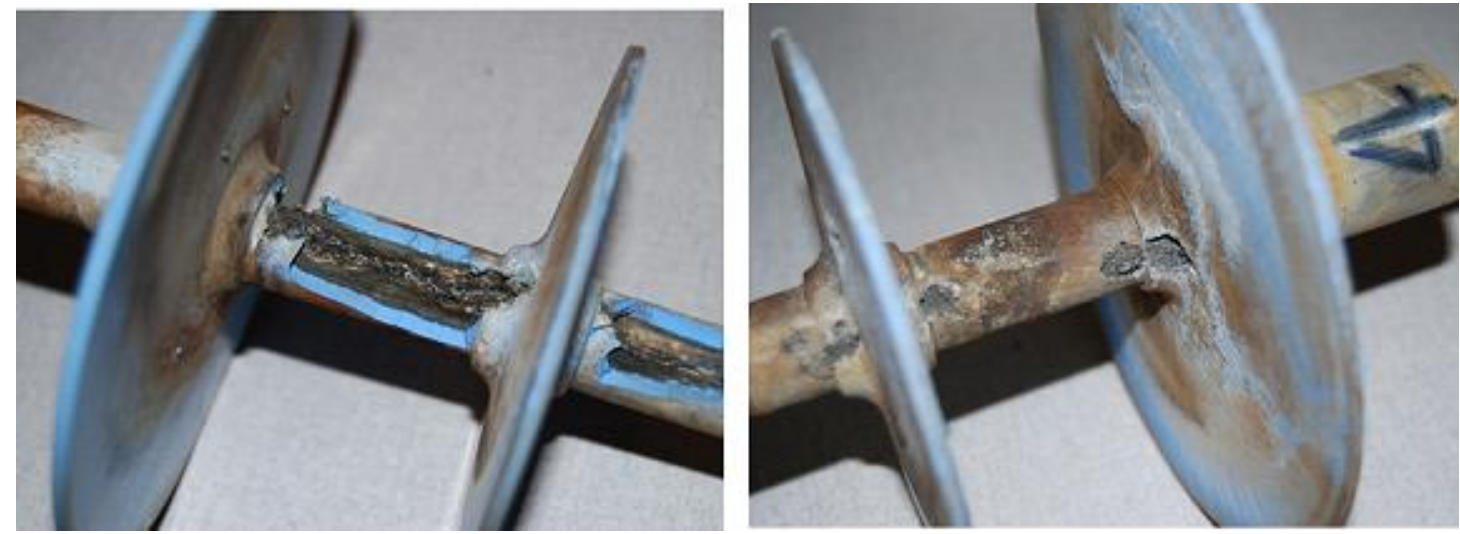

Figure 4.57: Comparison of aging of the HTV SR insulators with unified SCD of $28 \mathrm{~mm} / \mathrm{kV}$ and $35 \mathrm{~mm} / \mathrm{kV}$ for positive polarity HVDC excitation

Figure 4.58 shows the porcelain insulator (Channel 5) before and after the test, while Figure 4.59 shows the aging in more detail. This insulator shows brown discoloration but did not age much. The insulator experienced no material degradation.
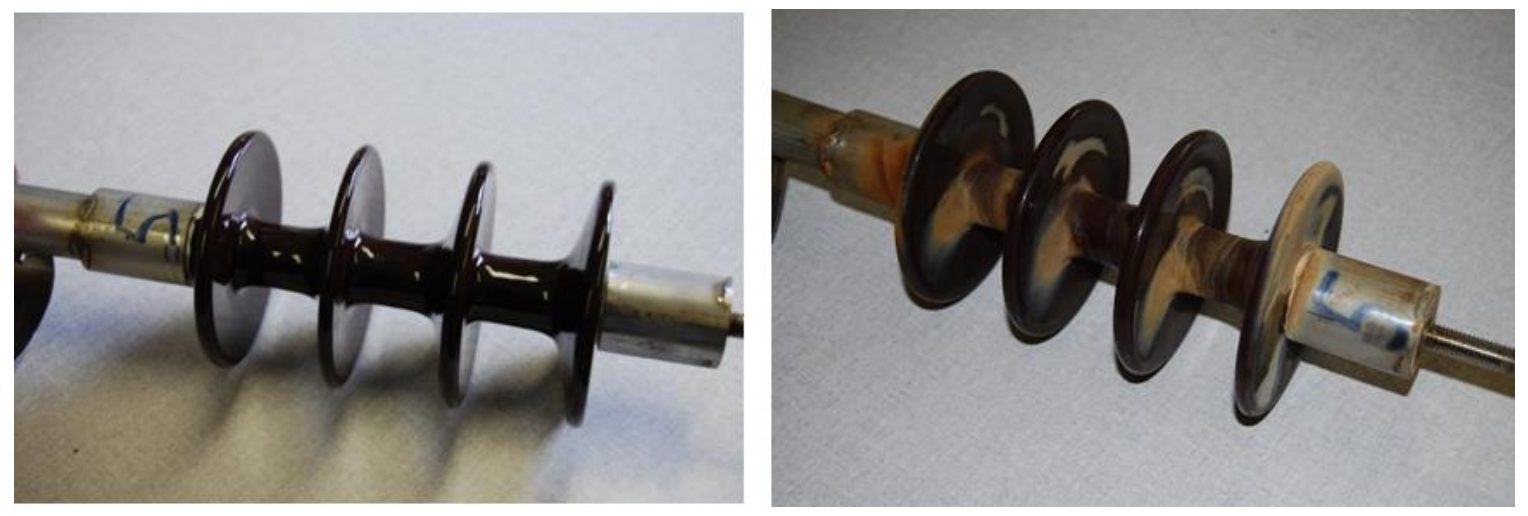

Figure 4.58: The porcelain insulator (Channel 5) before and after the test with positive polarity HVDC excitation 


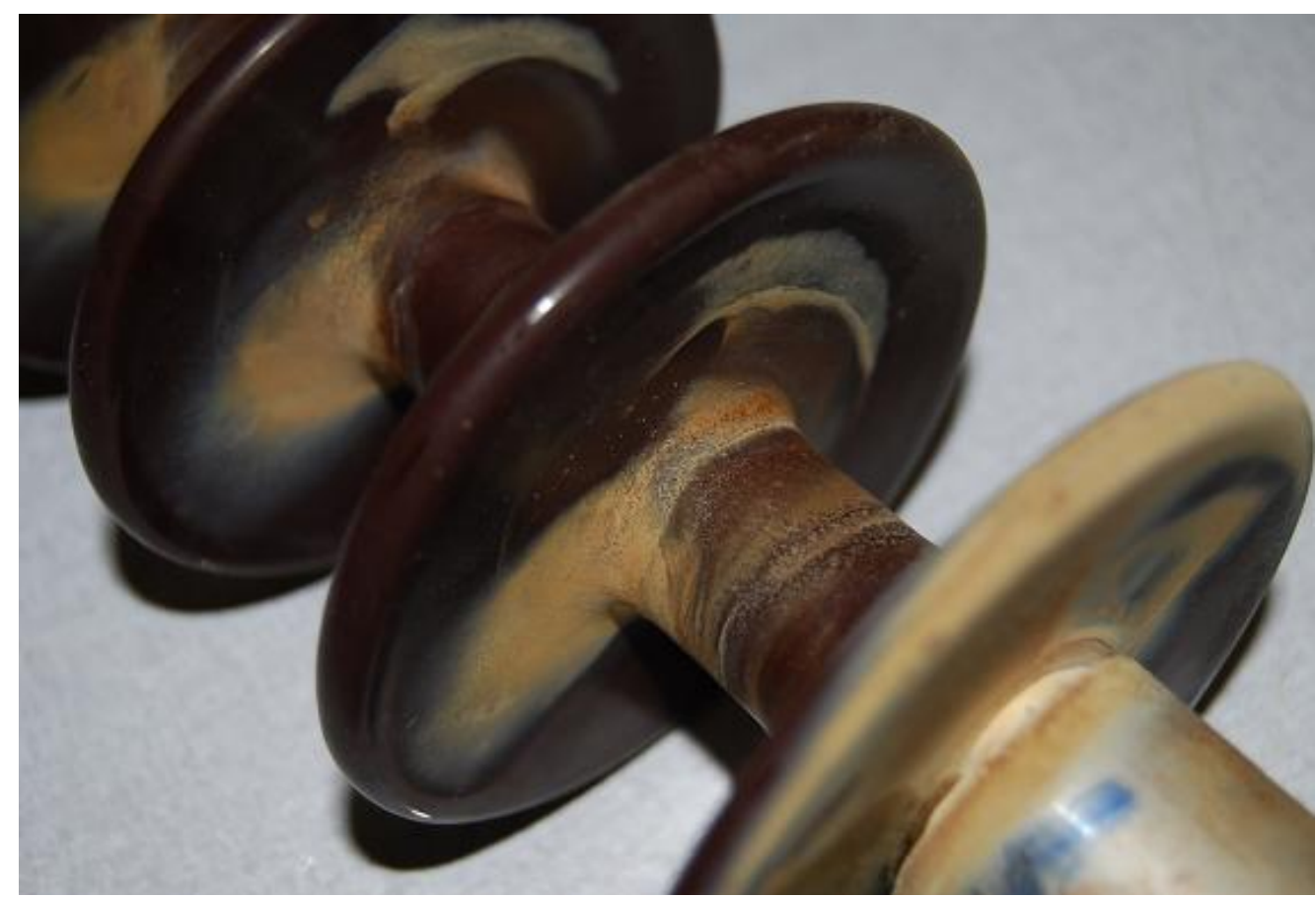

Figure 4.59: Detailed view of aging of the porcelain insulator (Channel 5) for positive polarity HVDC excitation

Figure 4.60 shows the RTV SR coated porcelain insulator (Channel 6) before and after the test, while Figure 4.61 shows the aging in more detail. The aging of the insulator is clearly evident. The skin had eroded away, especially on the rods where the material was completely cleared off. There was not that much erosion on the sheds, even though there was a lot of discoloration on the insulator itself. There is evidence of a white discoloration.
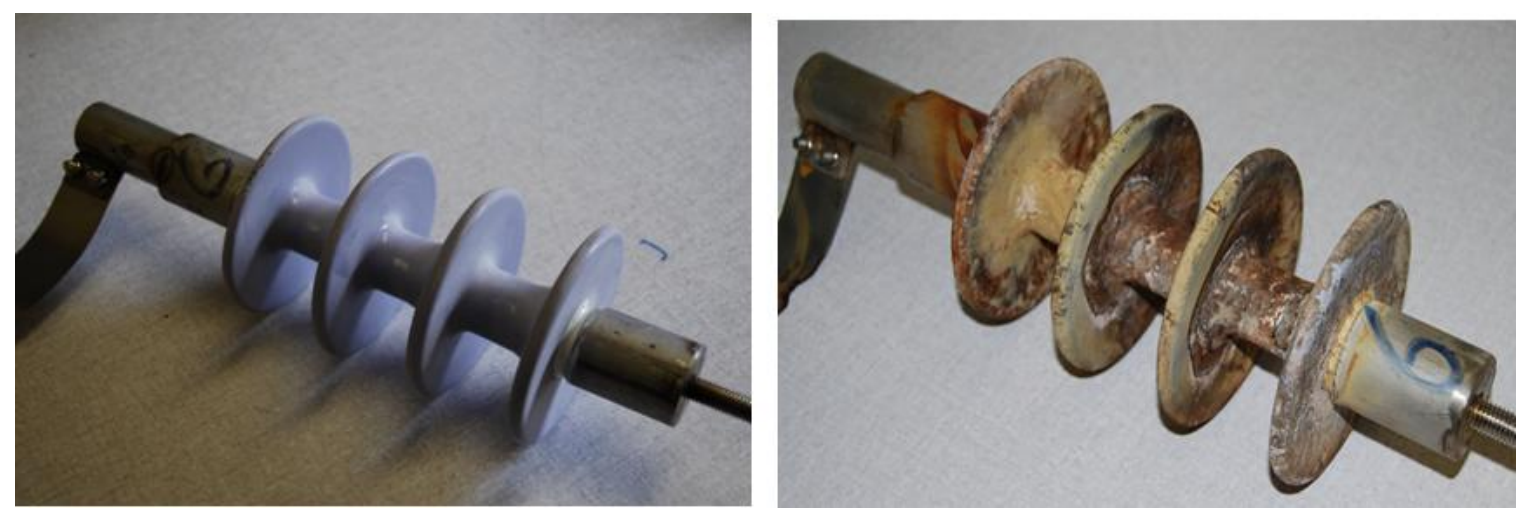

Figure 4.60: The RTV SR coated porcelain insulator (Channel 6) before and after the test with positive polarity HVDC excitation 


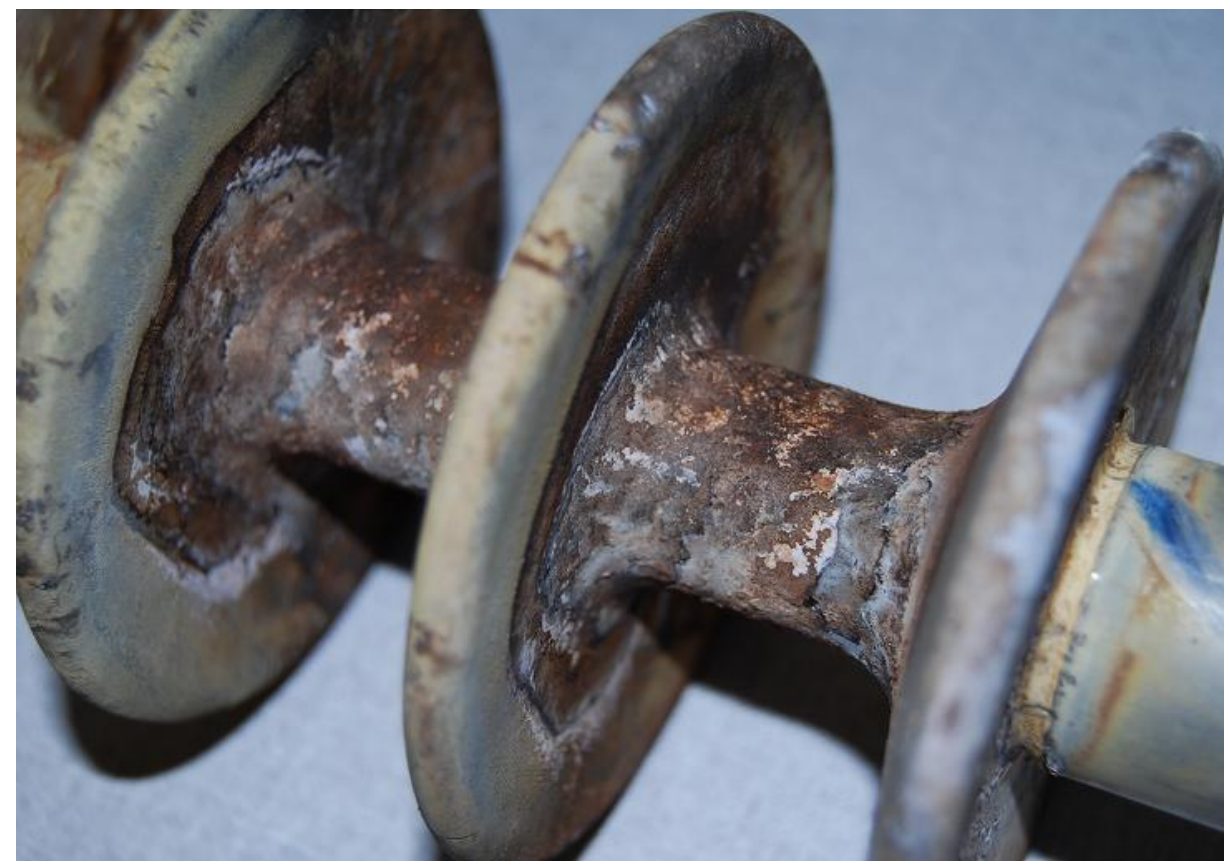

Figure 4.61: Detailed view of aging of the RTV SR coated porcelain insulator (Channel 6) for positive polarity HVDC excitation

Figure 4.62 shows the porcelain (Channel 5) and RTV coated porcelain (Channel 6) insulators. The porcelain insulator (Channel 5) only had a slight brown discoloration and did not age much. The RTV SR coated porcelain insulator (Channel 6) seemed to have aged faster since it had severe discoloration and erosion, as well as flaking of the coating. The RTV SR coated porcelain insulator (Channel 6) seems to have aged faster compared to the porcelain insulator (Channel 5).
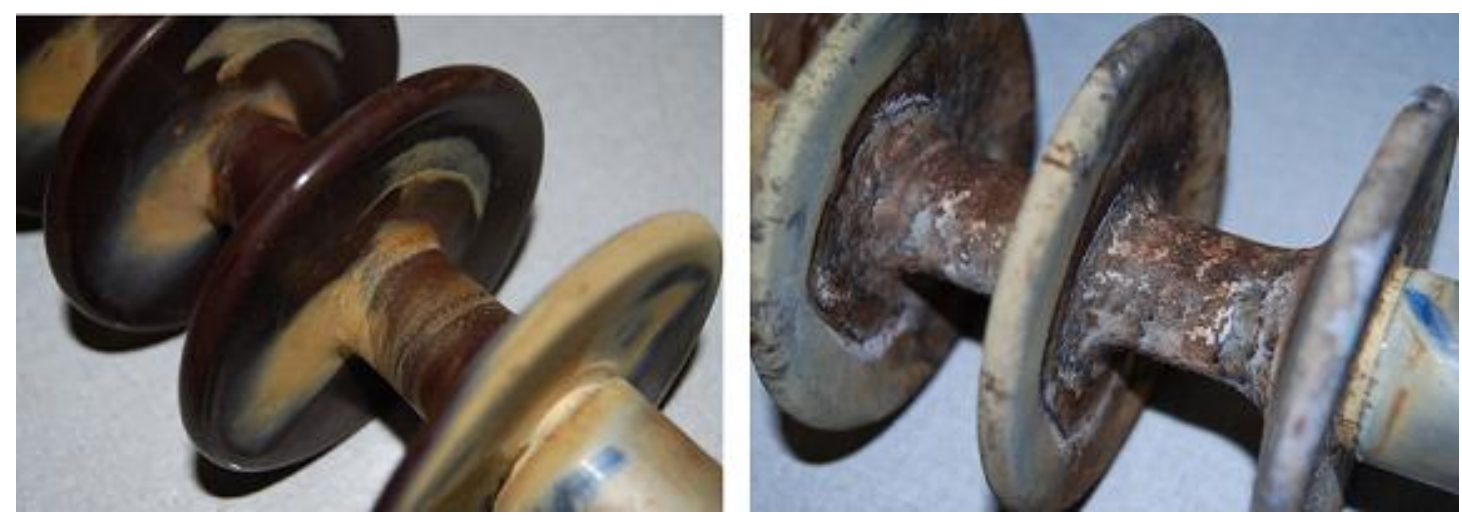

Figure 4.62: Comparison of aging of the porcelain and RTV SR coated porcelain for positive polarity HVDC excitation

\section{Hydrophobic properties}

Figure 4.63 compares the hydrophobicity of the EPDM insulator (Channel 1) and the HTV SR insulator (Channel 2). The EPDM insulator has a wettability class of 5, 
while the HTV SR insulator has a wettability class of 3. In other words, the EPDM insulator seems to have lost hydrophobicity, while the HTV SR insulator recovered hydrophobicity.
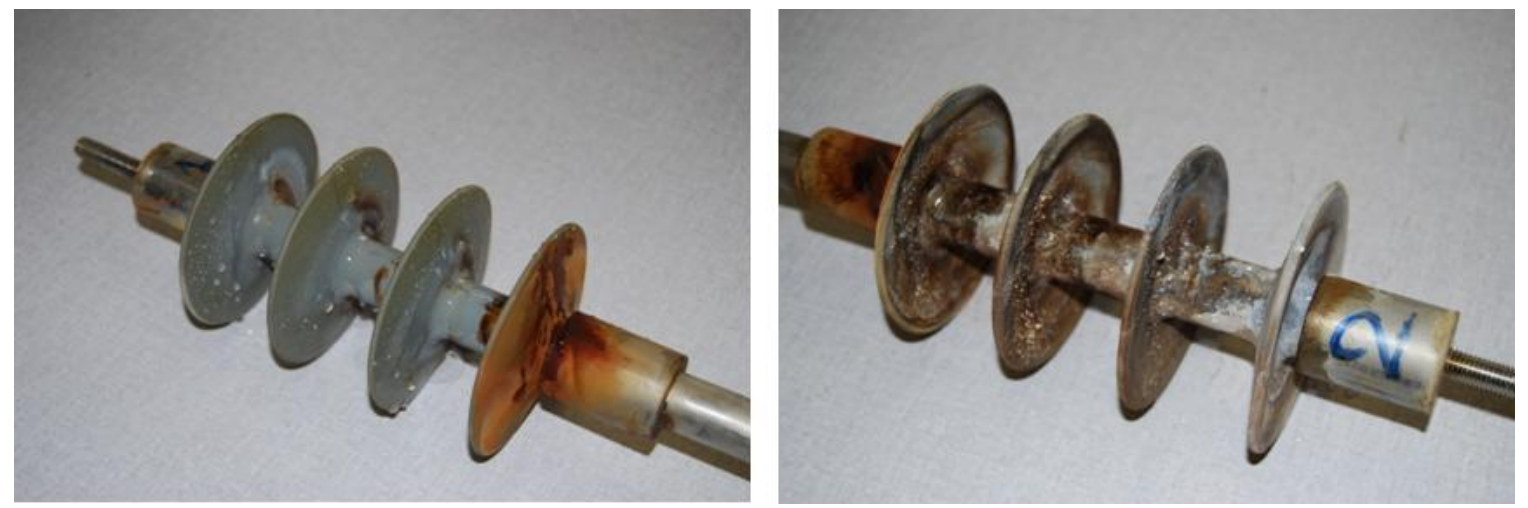

Figure 4.63: Wettability of the EPDM insulator (Channel 1) and the HTV SR insulator (Channel 2), both with a unified SCD of $28 \mathrm{~mm} / \mathrm{kV}$

Figure 4.64 compares the hydrophobicity of the HTV SR insulators, both with a unified SCDof $28 \mathrm{~mm} / \mathrm{kV}$, from manufacturer A (Channel 2) and manufacturer B (Channel 3). The insulator from manufacturer A has a wettability class of 3 , while the insulator from manufacturer B has a wettability class of 4 . Thus, the insulator from manufacturer B retained hydrophobicity better than insulator from manufacturer A.
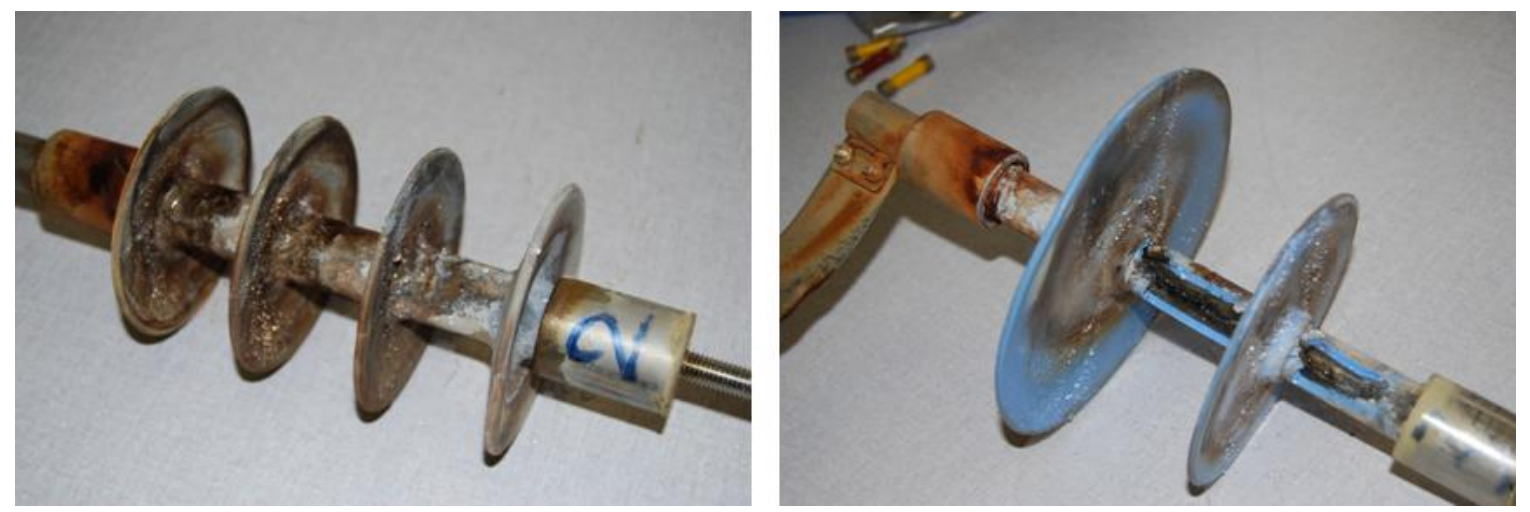

Figure 4.64: Wettability of the HTV SR insulators from manufacturer A (Channel 2) and manufacturer B (Channel 3), both with a unified SCD of $28 \mathrm{~mm} / \mathrm{kV}$

Figure 4.65 compares the hydrophobicity of the HTV SR insulator with a unified SCD of $28 \mathrm{~mm} / \mathrm{kV}$ (Channel 3) with the HTV SR insulator a unified SCDof $35 \mathrm{~mm} / \mathrm{kV}$ (Channel 4). Channel 3 has a wettability class of 4, while Channel 4 has a wettability class of 3 to 4 . Thus the HTV SR insulator (Channel 4) with a longer unified SCDretained hydrophobicity better than the HTV SR insulator with a shorter unified SCD (Channel 3). 

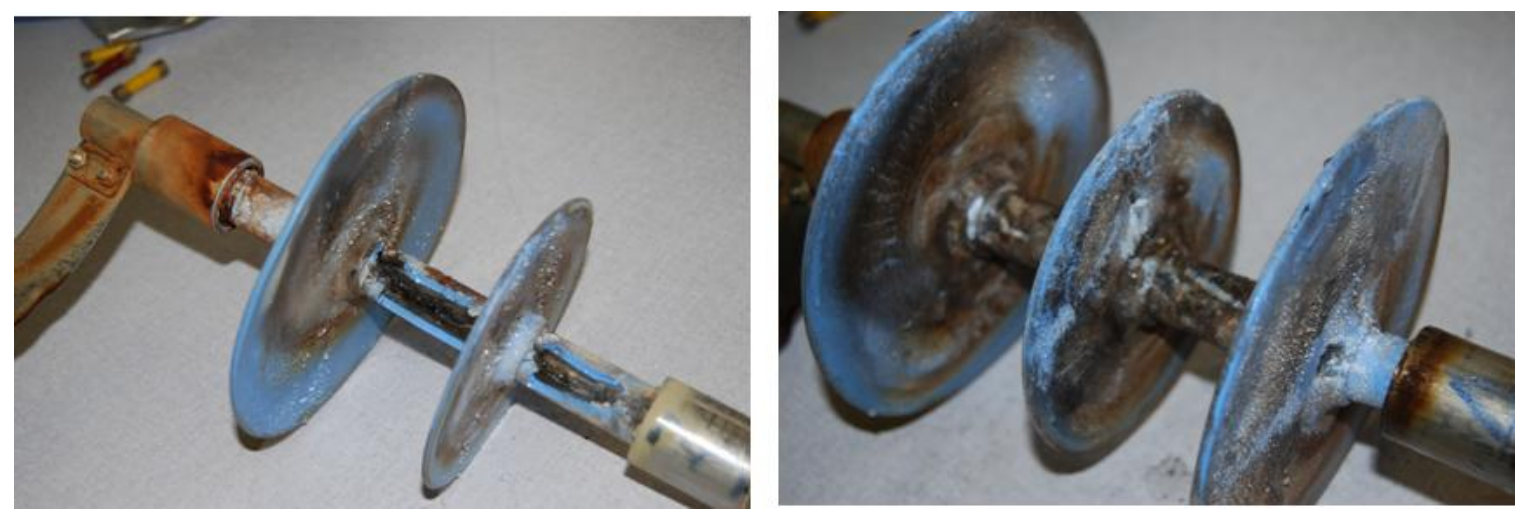

Figure 4.65: Wettability of the HTV SR insulator with a unified SCD of $28 \mathrm{~mm} / \mathrm{kV}$ (Channel 3) and the HTV SR insulator with a unified SCD of $35 \mathrm{~mm} / \mathrm{kV}$ (Channel 4)

Figure 4.66 compares the hydrophobicity of the porcelain insulator (Channel 5) with the RTV SR coated porcelain insulator (Channel 6). The porcelain Channel 5 is completely hydrophilic, since it has a wettability class of 6 , while Channel 6 has a wettability class of 4 to 5 . Thus, the RTV SR coated insulator (Channel 6) seems to retain its hydrophobicity better than the porcelain insulator (Channel 5).
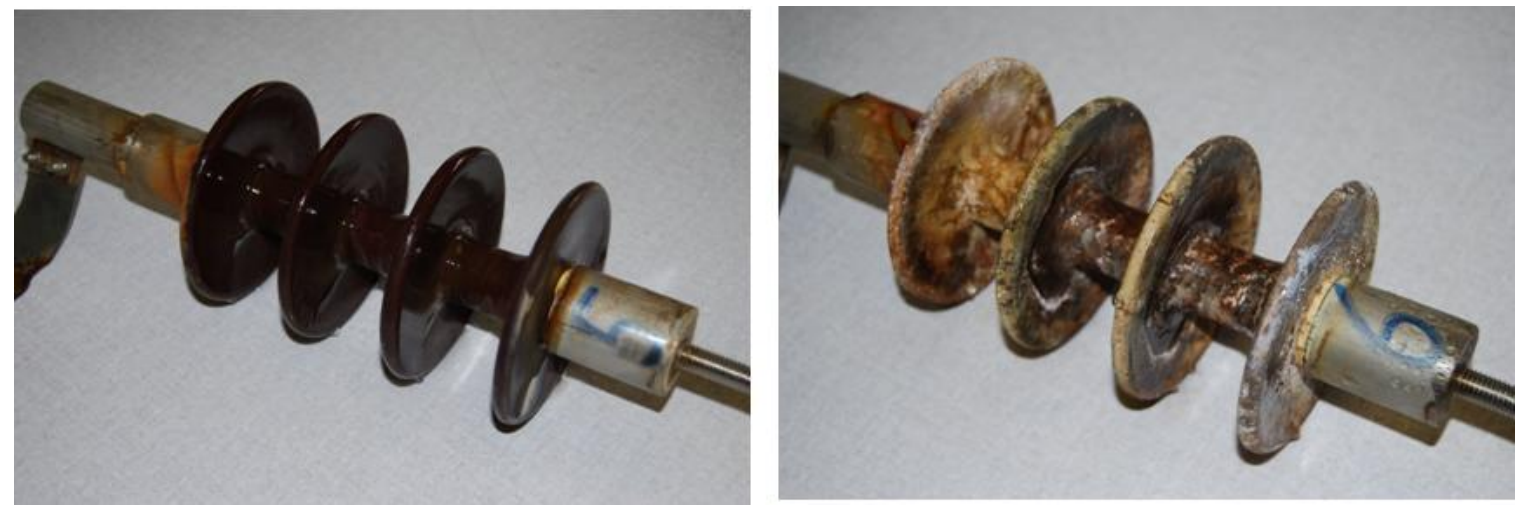

Figure 4.66: Wettability of the porcelain insulator (Channel 5) and the RTV SR coated porcelain insulator (Channel 6)

\subsection{Second test series: Negative polarity HVDC excitation}

\subsubsection{Overview}

The second series of test was repeated with for the six insulator samples shown in Table 4.4, using negative polarity HVDC excitation. This experiment was also based on a combination of the IEC 61302 and the IEC 62217 (Annex A) standards. The experiment was started on the $15^{\text {th }}$ July 2008 at around 09:47, and it was stopped on the $1^{\text {st }}$ September 2008. 


\subsubsection{Test methodology and test procedures}

The insulators were installed in exactly the same way as for the tests with HVAC and positive polarity HVDC excitation. This experiment used the same methodology and operating procedures as for the positive polarity HVDC excitation. The voltage applied was $13.6 \mathrm{kV} \mathrm{dc}$, but with the rectifiers connected in the opposite direction, and the conductivity used was $2.43 \mathrm{mS} / \mathrm{cm}$ as before. Table 4.4 summarizes the details of the samples tested with negative polarity HVDC excitation. The same method used for the third experiment in section 4.3.1 applies. Thus, the HVDC supply was now being used in the negative direction.

\subsubsection{Summary of test results}

\subsubsection{Leakage current}

Table 4.12 and Figure 4.67 summarize the leakage current results for the EPDM (Channel 1) and HTV SR (Channel 2) insulators. At the beginning of the experiment the EPDM insulator (Channel 1) had a peak current of $21 \mathrm{~mA}$ while the HTV SR insulator (Channel 2) had a peak current of $18 \mathrm{~mA}$. After one week, however, the HTV SR insulator lost hydrophobicity. This gave rise to higher peak currents for HTV SR insulator compared to the EPDM insulator. At the end of the experiment the EPDM insulator (Channel 1) had a peak current of approximately $50 \mathrm{~mA}$ while HTV SR insulator (Channel 2) had a peak current of $110 \mathrm{~mA}$.

Table 4.12: Peak leakage currents for Channel 1 and channel 2 for negative polarity HVDC excitation

\begin{tabular}{|l|l|l|}
\hline $\begin{array}{l}\text { Peak current [mA] in the } \\
\text { beginning }\end{array}$ & Channel 1 (EPDM insulator) & Channel 2 (HTV SR insulator) \\
\hline Peak current $[\mathrm{mA}]$ in the end & $50 \mathrm{~mA}$ & $18 \mathrm{~mA}$ \\
\hline
\end{tabular}




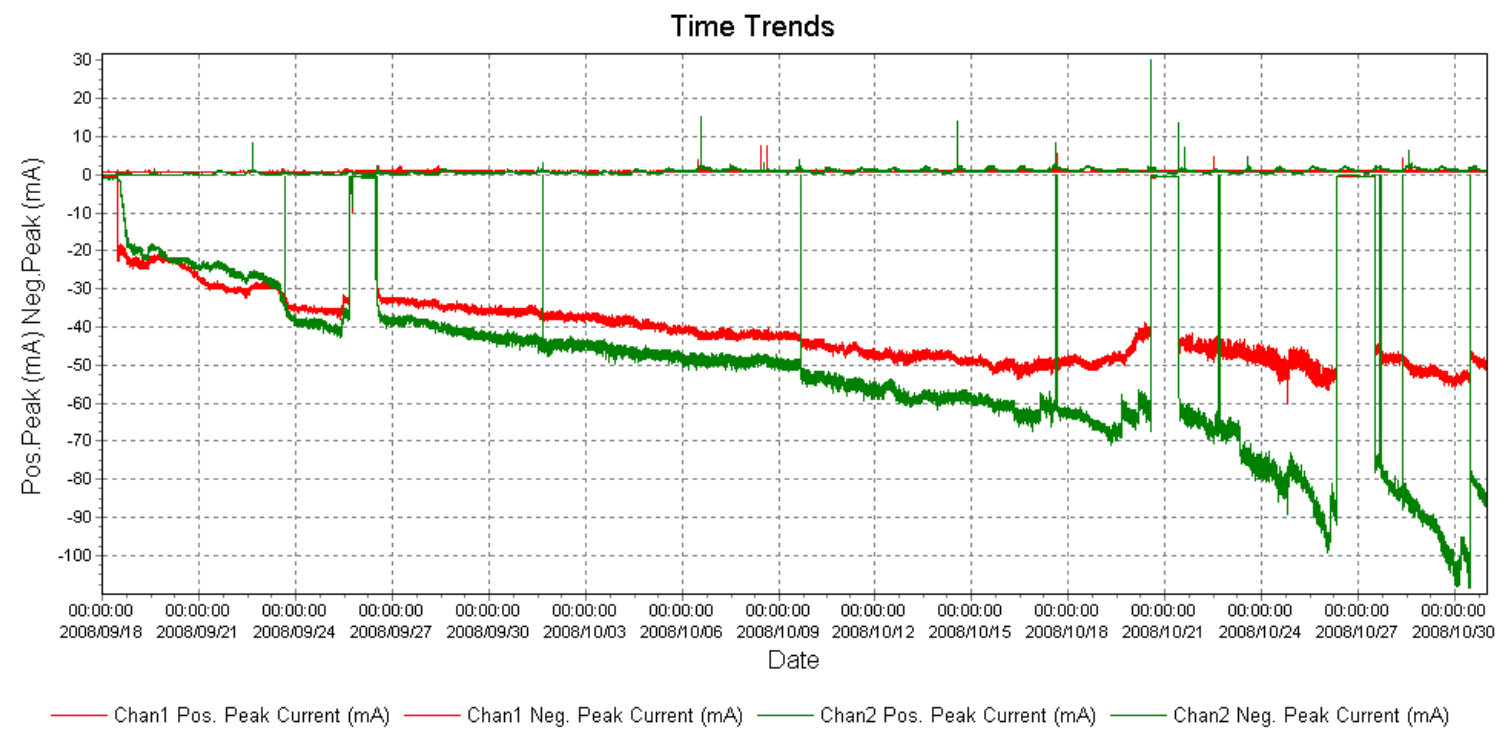

Figure 4.67: Peak leakage currents for the EPDM insulator (Channel 1) and HTV SR insulator (Channel 2) for negative HVDC negative excitation

Table 4.13 and Figure 4.68 summarize the leakage current results for the HTV SR insulator from manufacturer A (Channel 2) and HTV SR insulator from manufacturer B (Channel 3). At the beginning of the experiment the HTV SR insulator from manufacturer A (Channel 2) had a peak current of $18 \mathrm{~mA}$ while the HTV SR insulator from manufacturer B (Channel 3) had a peak current of $20 \mathrm{~mA}$. Channel 3 took about three days before any arcs or discharges appeared on the insulator, while Channel 2 developed arcs or discharges almost immediately. After 4 weeks, Channel 3 began to have higher peak currents when compared to Channel 2. The peak currents for Channel 3 continued to rise, until it eventually tripped on the $26^{\text {th }}$ October 2008 at 544 mA. Towards the end of the test, HTV SR insulator from manufacturer A (Channel 2) had a peak current of approximately $110 \mathrm{~mA}$, dropping down to $90 \mathrm{~mA}$ in the end. Initially the HTV SR insulator from manufacturer A had higher leakage currents compared to the HTV SR insulator from manufacturer B, but in the end this trend was reversed.

Table 4.13: Peak leakage currents for Channel 2 and Channel 3 for negative polarity HVDC excitation

\begin{tabular}{|l|l|l|}
\hline & $\begin{array}{l}\text { Channel 2 (HTV SR insulator } \\
\text { from manufacturer A) }\end{array}$ & $\begin{array}{l}\text { Channel 3 (HTV SR insulator } \\
\text { from manufacturer B) }\end{array}$ \\
\hline $\begin{array}{l}\text { Peak current }[\mathrm{mA}] \text { in the } \\
\text { beginning }\end{array}$ & $18 \mathrm{~mA}$ & $20 \mathrm{~mA}$ \\
\hline Peak current $[\mathrm{mA}]$ in the end & $\begin{array}{l}\text { Reaches a maximum of 110 } \\
\mathrm{mA} \text { and then drops to 90 mA in } \\
\text { the end }\end{array}$ & $\begin{array}{l}544 \mathrm{~mA} \\
\text { "tripped" }\end{array}$ \\
\hline
\end{tabular}




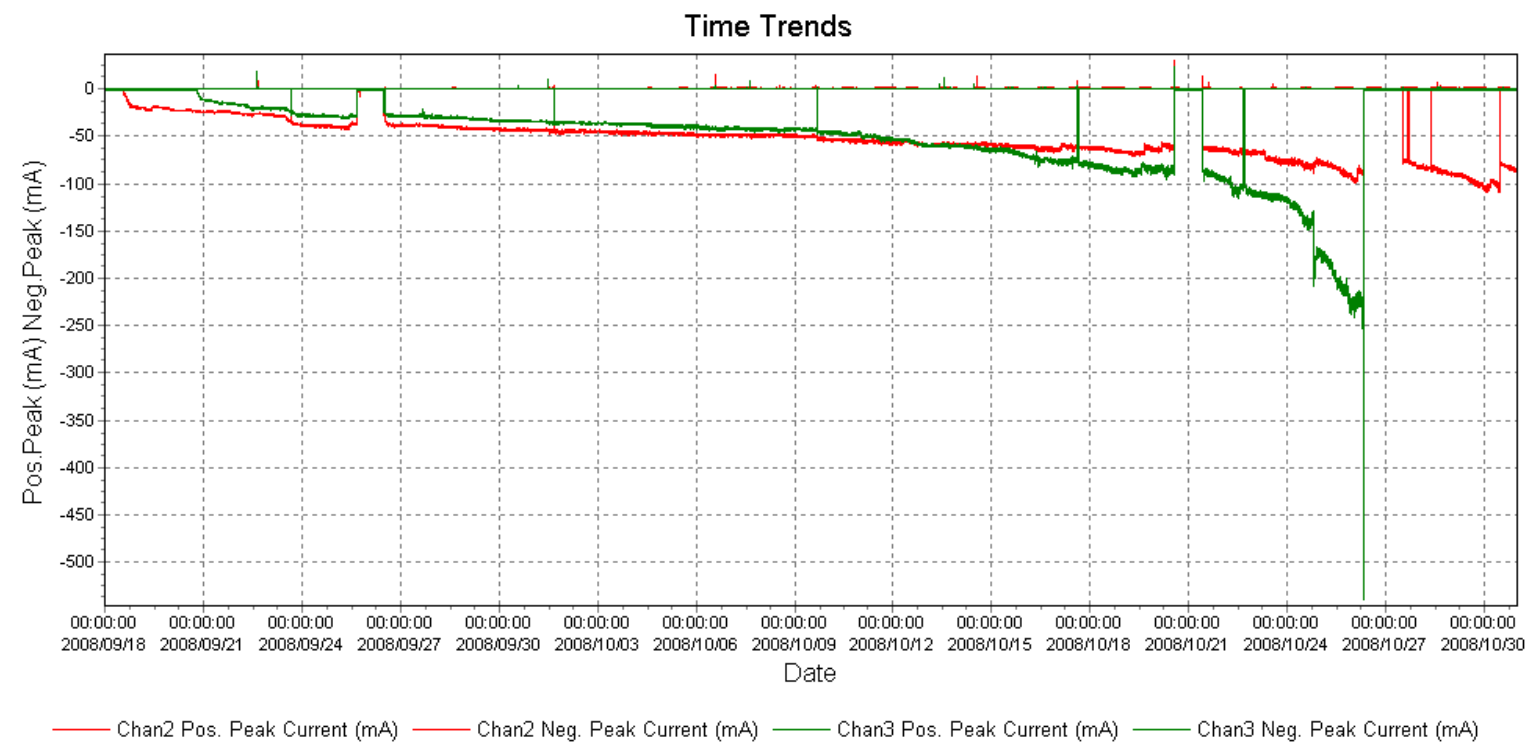

Figure 4.68: Peak leakage currents for the HTV SR insulators from manufacturer A (Channel 2) and from manufacturer B (Channel 3) for negative HVDC negative excitation

Table 4.14 and Figure 4.69 summarize the leakage current results for the HTV SR insulator with a unified SCDof $28 \mathrm{~mm} / \mathrm{kV}$ (Channel 3) and the HTV SR insulator with a unified SCDof $35 \mathrm{~mm} / \mathrm{kV}$ (Channel 4). At the beginning of the experiment the HTV SR insulator with a unified SCDof $28 \mathrm{~mm} / \mathrm{kV}$ (Channel 3) had a peak current of $8 \mathrm{~mA}$ while the HTV SR insulator with a unified SCDof $35 \mathrm{~mm} / \mathrm{kV}$ (Channel 4) had a peak current of $12 \mathrm{~mA}$. However towards the end, Channel 3 still had a higher peak currents compared to Channel 4. Thus, the insulator with the shortest unified SCD (Channel 3) had higher peak currents compared to the insulator with the longest unified SCD (Channel 4) throughout the duration of the experiment. Channel 3 tripped on the $26^{\text {th }}$ October 2008 at a peak current of approximately $544 \mathrm{~mA}$. Channel 4 tripped on the $8^{\text {th }}$ November 2008 at a peak current of approximately $700 \mathrm{~mA}$.

Table 4.14: Peak leakage currents for Channel 3 and Channel 4 for negative polarity HVDC excitation

\begin{tabular}{|l|l|l|}
\hline & $\begin{array}{l}\text { Channel 3 (HTV SR insulator } \\
\text { with USCDof 28 } \mathbf{~ m m} / \mathbf{k V})\end{array}$ & $\begin{array}{l}\text { Channel 4 (HTV SR insulator } \\
\text { with USCDof 35 } \mathbf{~ m m} / \mathbf{k V})\end{array}$ \\
\hline $\begin{array}{l}\text { Peak current [mA] in the } \\
\text { beginning }\end{array}$ & $8 \mathrm{~mA}$ & $12 \mathrm{~mA}$ \\
\hline Peak current [mA] in the end & Tripped at 544 mA after 38 days & Tripped at 700 mA after 43 days \\
\hline Hydrophobicity loss & 3 days & 3 days and some hours \\
\hline
\end{tabular}




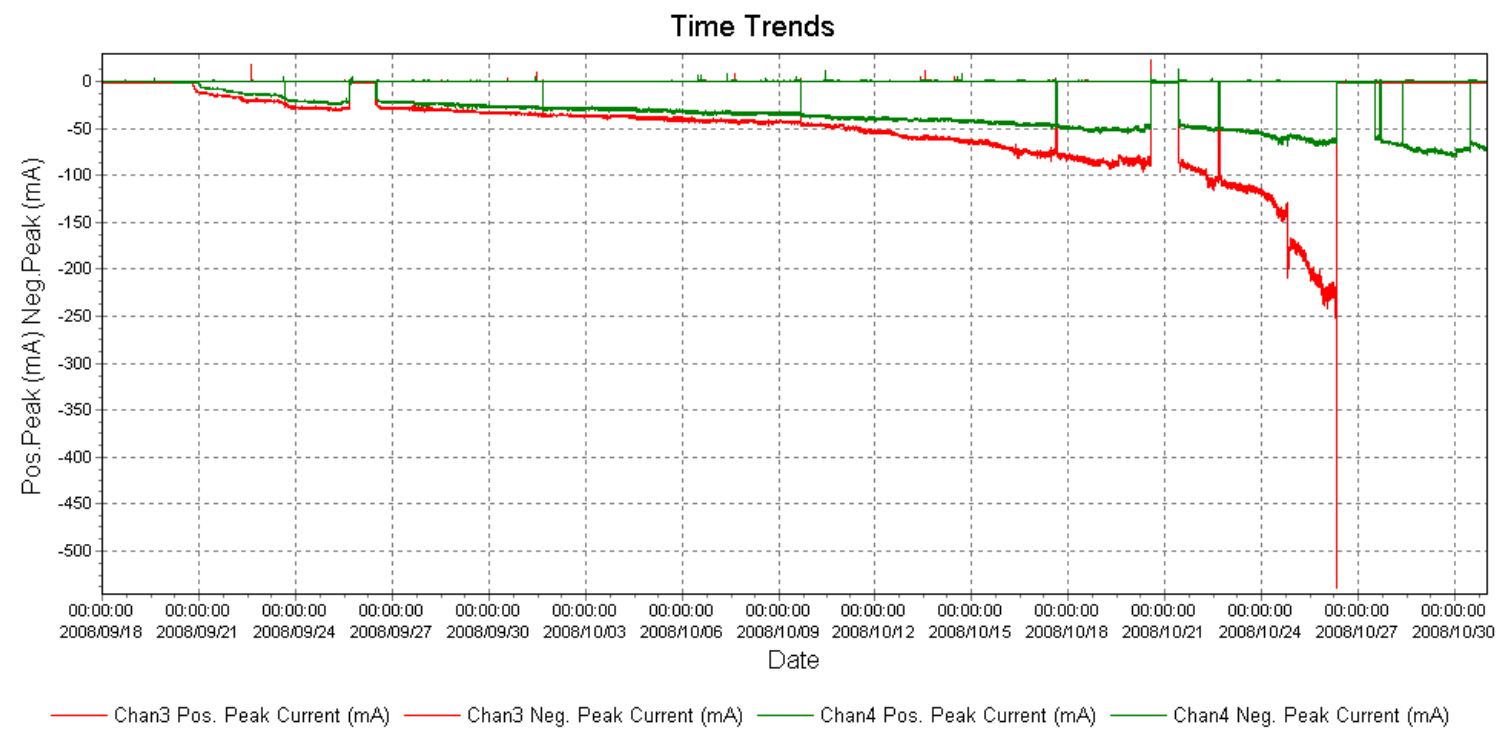

Figure 4.69: Peak leakage currents for the HTV SR insulators with a unified SCDof 28 $\mathrm{mm} / \mathrm{kV}$ (Channel 3) and a unified SCDof $35 \mathrm{~mm} / \mathrm{kV}$ (Channel 4) for negative polarity HVDC excitation

Table 4.15 and Figure 4.70 summarize the leakage current results for the reference porcelain insulator (Channel 5) and the RTV SR coated porcelain insulator (Channel 6). At the beginning of the experiment the porcelain insulator (Channel 5) had a peak current of $17 \mathrm{~mA}$ while the RTV SR coated porcelain insulator (Channel 6) had a peak current of $35 \mathrm{~mA}$. Once the voltage was applied, it took about two days before a leakage current appeared for the porcelain insulator, and about six days before a leakage current appeared for the RTV SR coated porcelain insulator. However, as the hydrophobicity property of the RTV SR coated porcelain insulator (Channel 6) was lost, this insulator showed a rise in peak leakage current, and end up having a higher peak current compared to porcelain insulator (Channel 5). The RTV SR coated porcelain insulator (Channel6) tripped on the $20^{\text {th }}$ October 2008 at peak current of 700 $\mathrm{mA}$. There porcelain insulator (Channel 5) had a peak current of $55 \mathrm{~mA}$ when the experiment was stopped.

Table 4.15: Peak leakage currents for Channel 5 and Channel 6 for negative polarity HVDC excitation

\begin{tabular}{|l|l|l|}
\hline & $\begin{array}{l}\text { Channel 5 (Porcelain } \\
\text { insulator) }\end{array}$ & $\begin{array}{l}\text { Channel 6 (RTV SR coated } \\
\text { porcelain insulator) }\end{array}$ \\
\hline $\begin{array}{l}\text { Peak current [mA] in the } \\
\text { beginning }\end{array}$ & $17 \mathrm{~mA}$ & $35 \mathrm{~mA}$ \\
\hline Peak current [mA] in the end & $55 \mathrm{~mA}$ & $\begin{array}{l}700 \mathrm{~mA} \\
\text { "tripped" }\end{array}$ \\
\hline Hydrophobicity lost & 2 days & 6 days \\
\hline
\end{tabular}




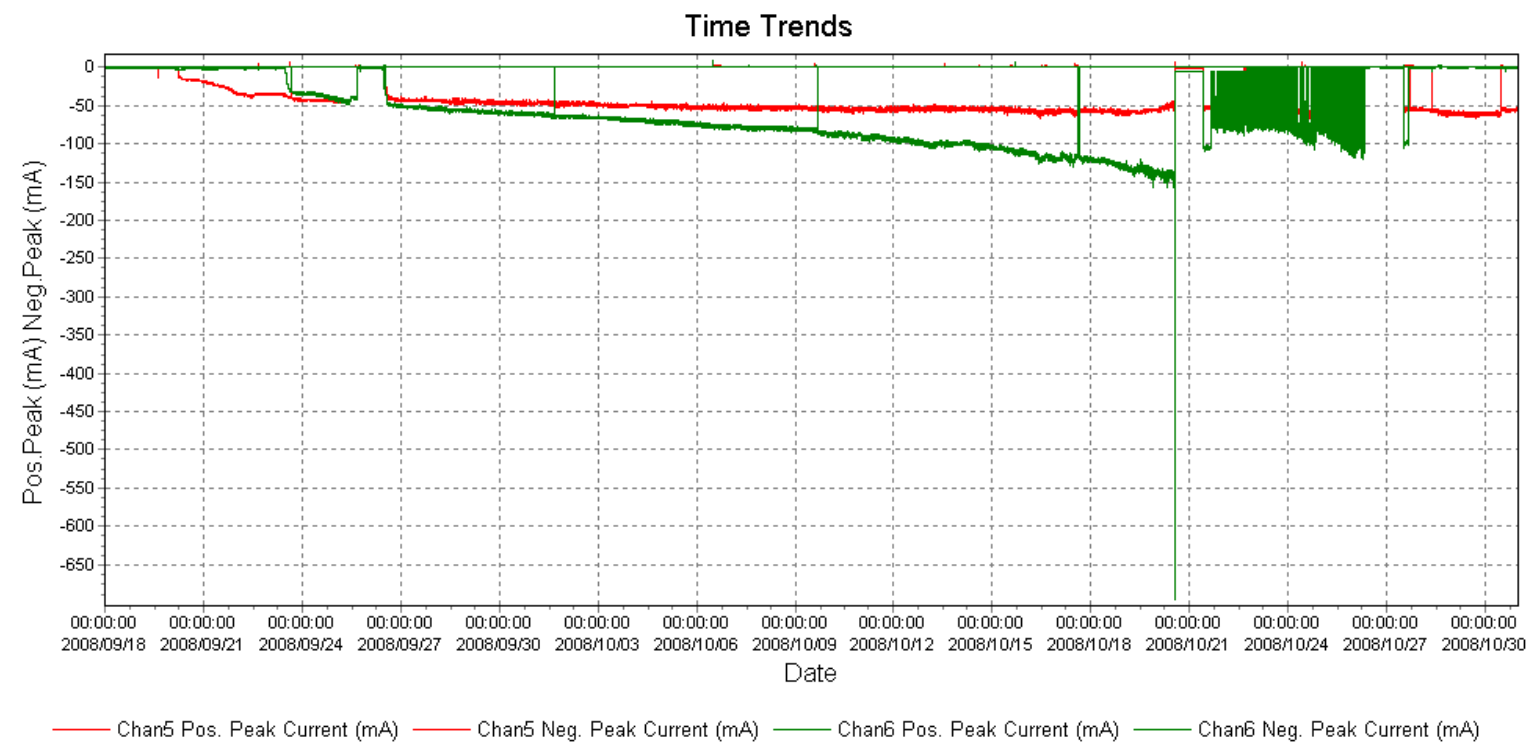

Figure 4.70: Peak leakage currents for the porcelain insulator (Channel 5) and the RTV SR coated porcelain insulator (Channel 6) for negative polarity HVDC excitation

\subsubsection{Visual observations of aging}

Visual observations were made on the test insulators on a daily basis with the view to determine how the insulators aged with time. Photographs and video recordings of the discharges of the insulators were taken on weekly basis.

Figure 4.71 shows the EPDM insulator with a unified SCD of $28 \mathrm{~mm} / \mathrm{kV}$ (Channel 1) before and after the test, while Figure 4.72 shows the aging in more detail. The insulator aged quite severely, and shows discoloration and blackening, especially on one side. The mould lines are completely disfigured with widening on the mould lines. There is some tearing on some of the EPDM sheds and some erosion.
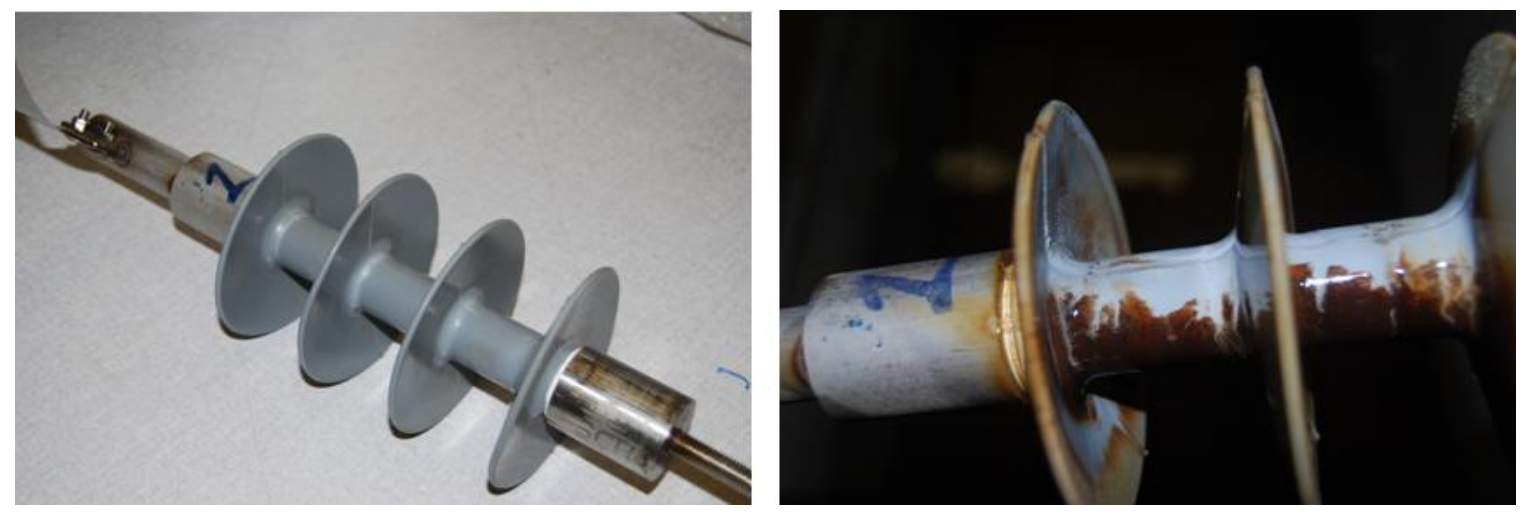

Figure 4.71: The EPDM insulator with a unified SCD of $28 \mathrm{~mm} / \mathrm{kV}$ (Channel 1) before and after the test with negative polarity HVDC excitation 


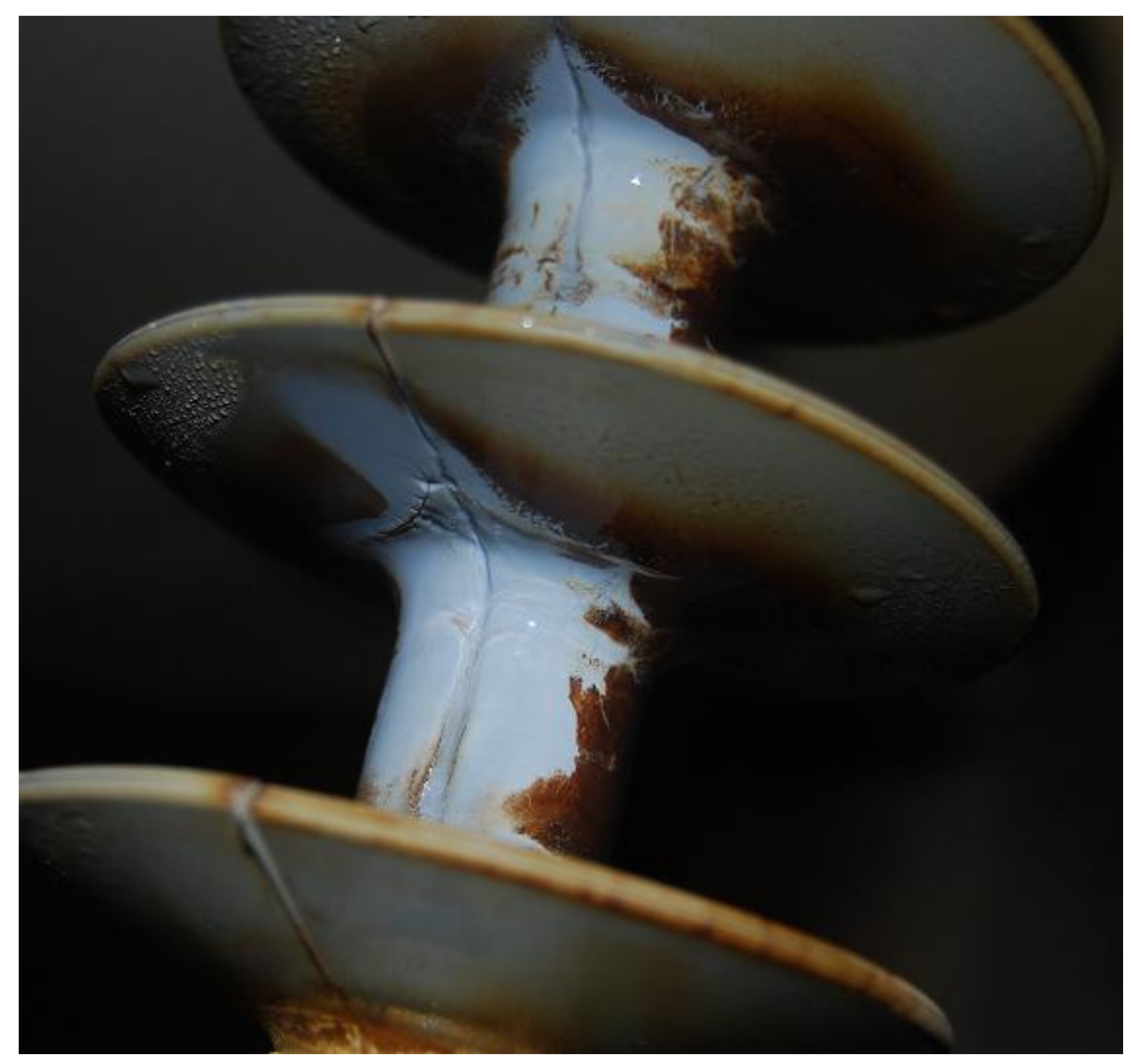

Figure 4.72: Detailed view of aging of the EPDM insulator (Channel 1) for negative polarity HVDC excitation

Figure 4.73 shows the HTV SR insulator with a unified SCD of $28 \mathrm{~mm} / \mathrm{kV}$ (Channel

2) before and after the test, while Figure 4.74 shows the aging in more detail. The insulator has tracks and erosion in the form of a star effect. The tracks appear on the pollution layer, with a little bit on the actual material. There is also a lot of discoloration on the sheds and rods.
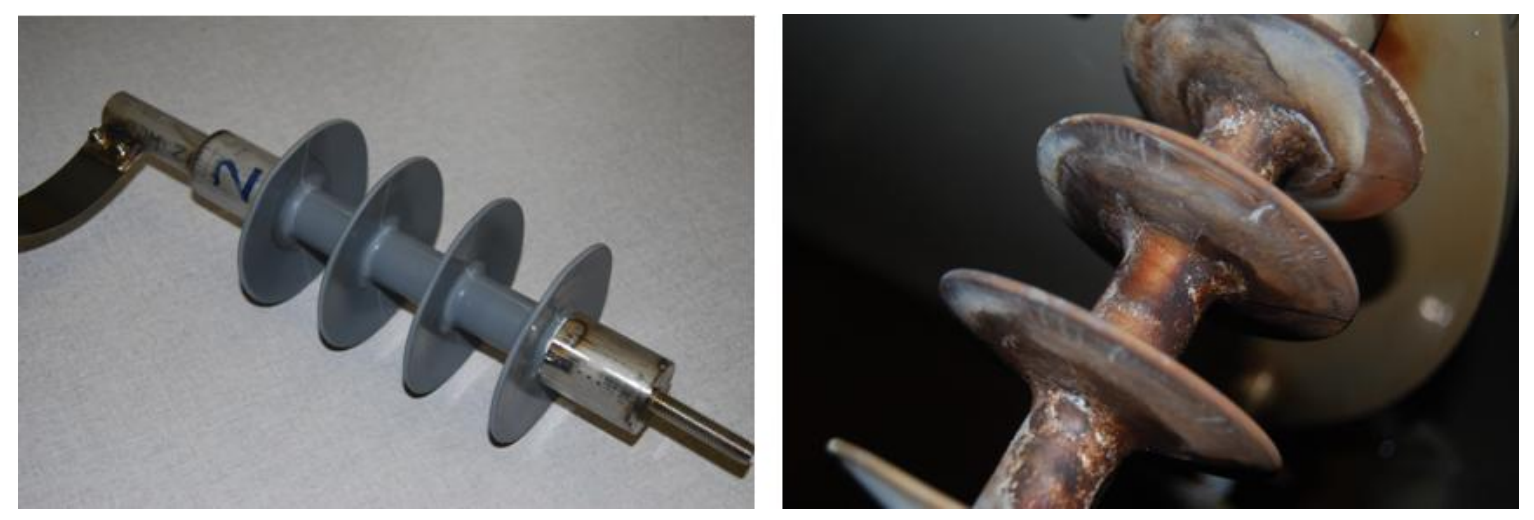

Figure 4.73: The HTV SR insulator with a unified SCD of $28 \mathrm{~mm} / \mathrm{kV}$ (Channel 2) before and after the test with negative polarity HVDC excitation 


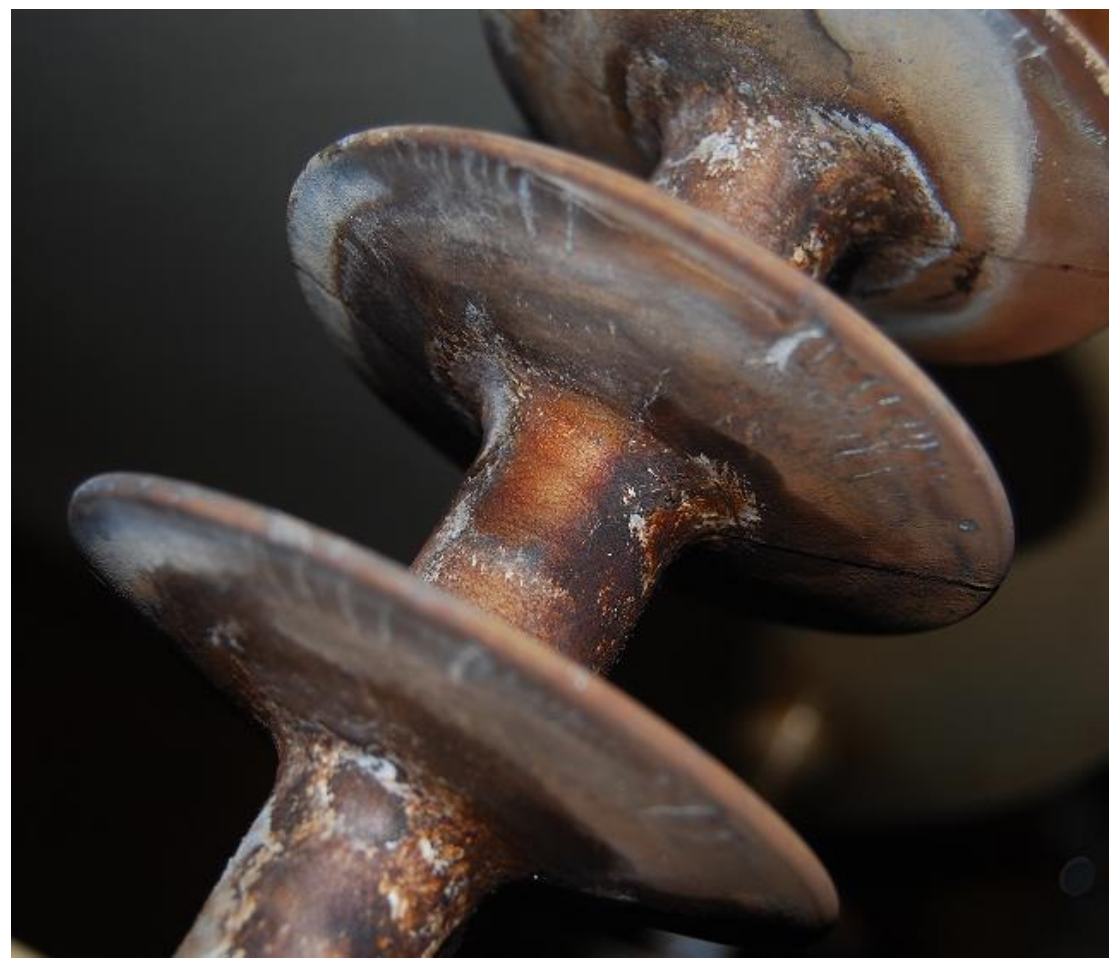

Figure 4.74: Detailed view of aging of the HTV SR insulator (Channel 2) for negative polarity HVDC excitation

Figure 4.75 compares the EPDM (Channel 1) and HTV SR (Channel 2) insulators. This diagram shows that the insulators aged differently, since the HTV SR insulator has discoloration on both sides, while the EPDM insulator has discoloration mostly on one side. The EPDM insulator had a deformation on the mould side, while the HTV SR insulator had no deformation on the mould side. Both Channel 1 and Channel 2 has erosion on the insulator. However, only the HTV SR insulator has tracks.
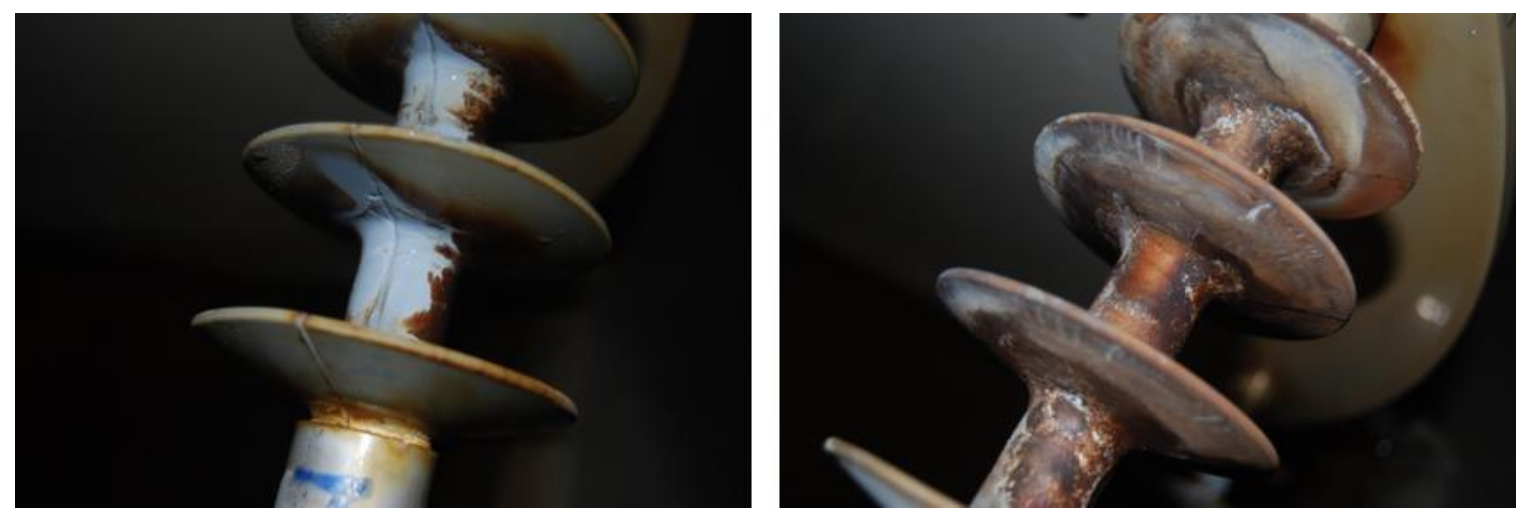

Figure 4.75: Channel 1 and Channel 2 after the test with negative polarity HVDC excitation

Figure 4.76 shows the HTV SR insulator with a unified SCD of $28 \mathrm{~mm} / \mathrm{kV}$ from manufacturer B (Channel 3) before and after the test, while Figure 4.74 shows the aging in more detail. The insulator shows discoloration and erosion, as well as severe 
tracks on the pollution layer in the form of a star effect. The insulator has four punctures, as well as burn marks on one side of the metal end fitting. A part of the rod is completely burned out and blackened.
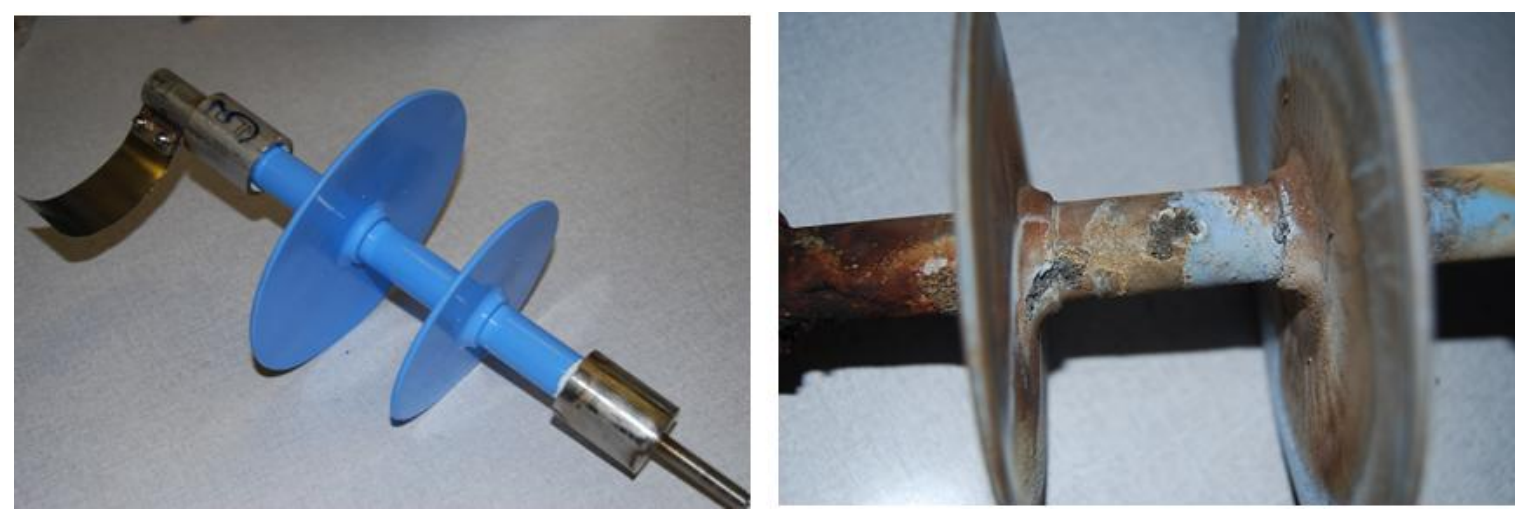

Figure 4.76: The HTV SR insulator with a unified SCD of $28 \mathrm{~mm} / \mathrm{kV}$ from manufacturer B (Channel 3) before and after the test with negative polarity HVDC excitation

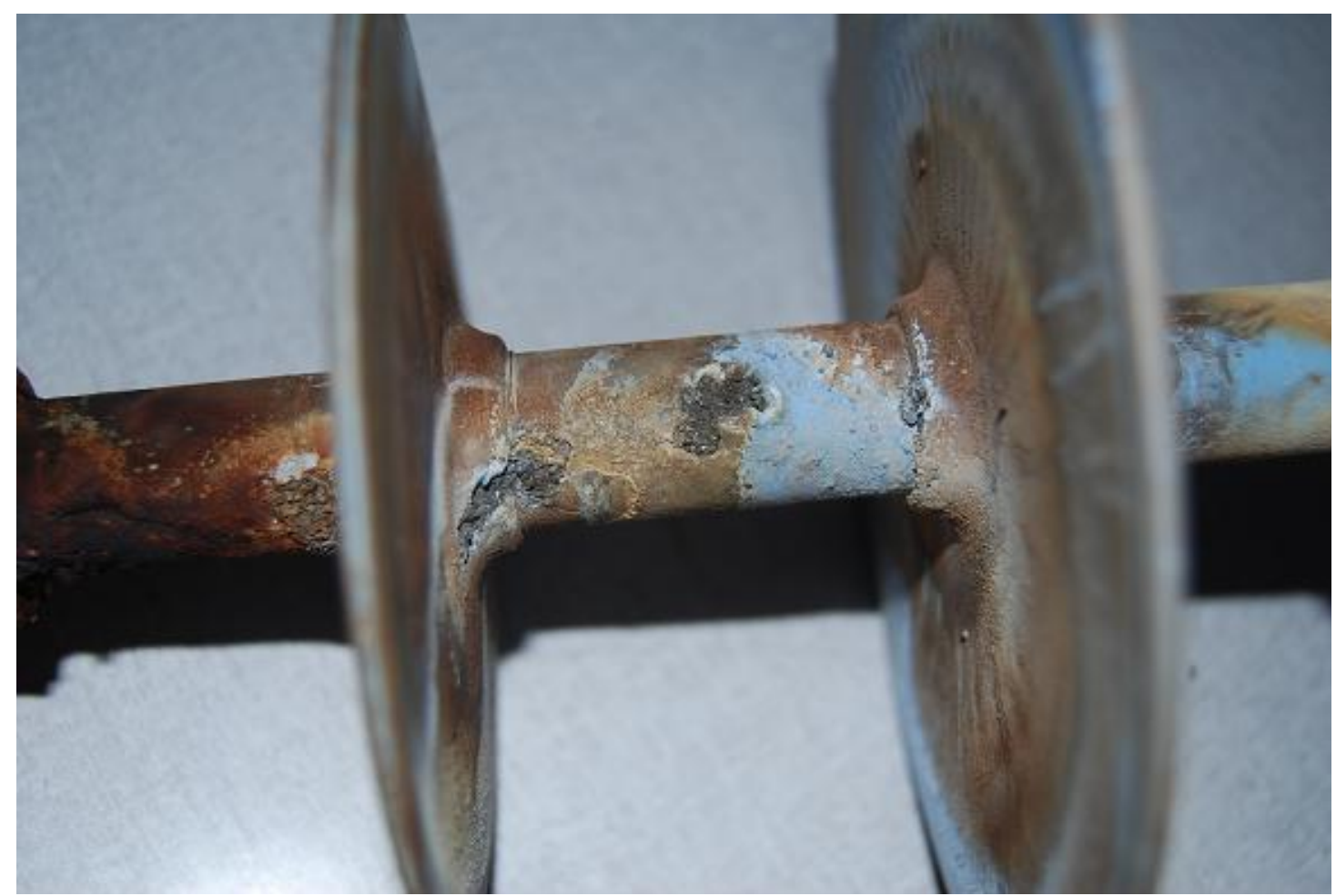

Figure 4.77: Detailed view of aging of the HTV SR insulator from manufacturer B (Channel 3) for negative polarity HVDC excitation

Figure 4.78 compares the HTV SR insulators with a unified SCDof $28 \mathrm{~mm} / \mathrm{kV}$ from manufacturer A (Channel 2) and manufacturer B (Channel 3). Channel 2 aged a little bit slower than Channel 3 since it had only one puncture, while Channel 3 had four punctures. Furthermore, Channel 3 had one side of the rod before the first shed completely burned out, while Channel 2 only had a slight burn at the metal end fitting. 
Both insulators had tracks in the form of a star effect on the pollution layer, with a little bit on the actual insulator.
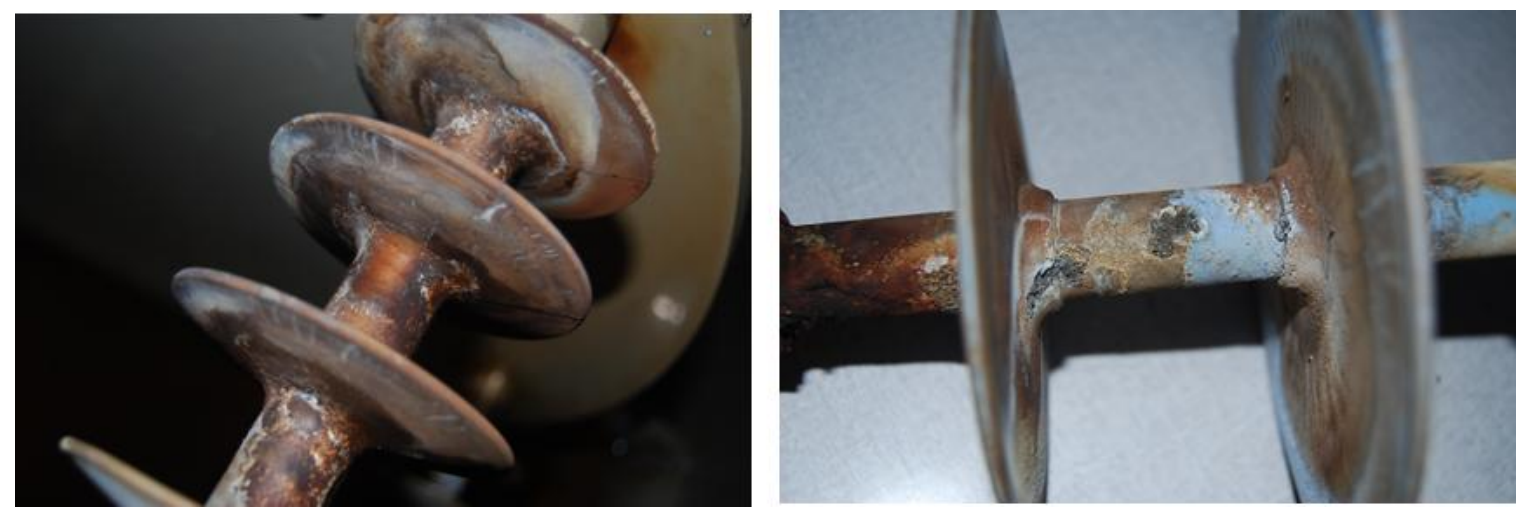

Figure 4.78: Channel 2 and Channel 3 after the test with negative polarity HVDC excitation

Figure 4.79 shows the HTV SR insulator with a unified SCD of $35 \mathrm{~mm} / \mathrm{kV}$ (Channel 4) before and after the test, while Figure 4.80 shows the aging in more detail. The insulator shows erosion, as well as the tracks on the sheds of the insulator. There is discoloration on the sheds and the rods. There insulator had tracks on the pollution layer in the form of a star effect. There are two punctures on the insulator and some of the material has worn away on the sheds and rods, as well as at the tips of the sheds.
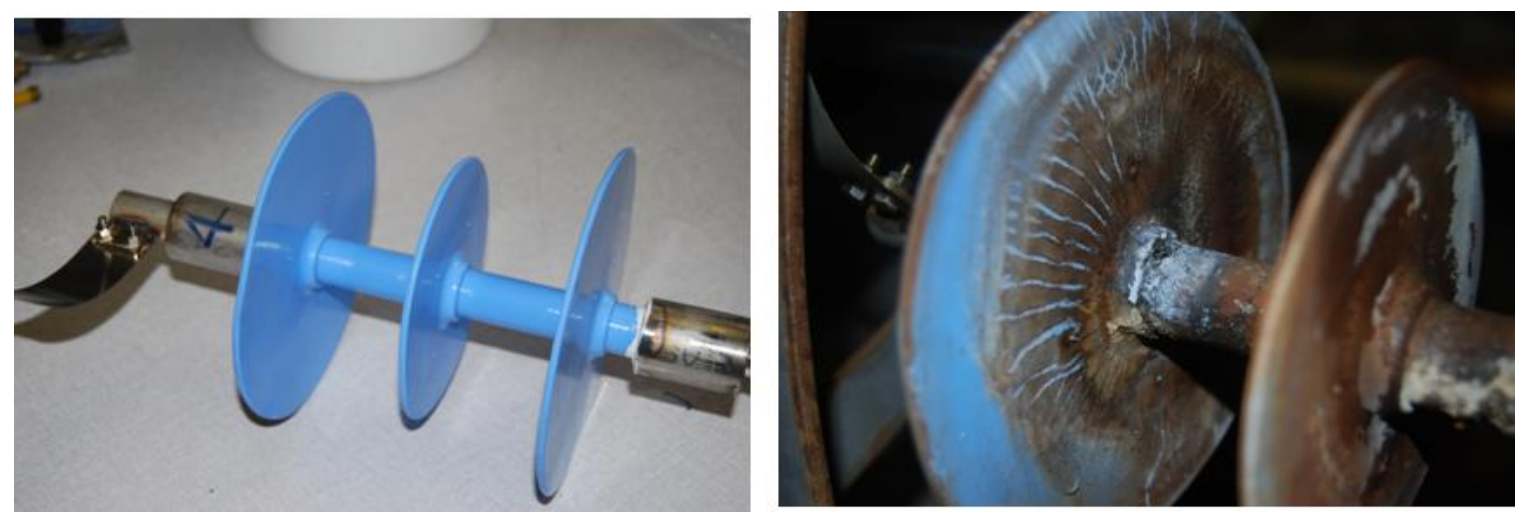

Figure 4.79: The HTV SR insulator with a unified SCD of $35 \mathrm{~mm} / \mathrm{kV}$ from manufacturer B (Channel 4) before and after the test with negative polarity HVDC excitation 


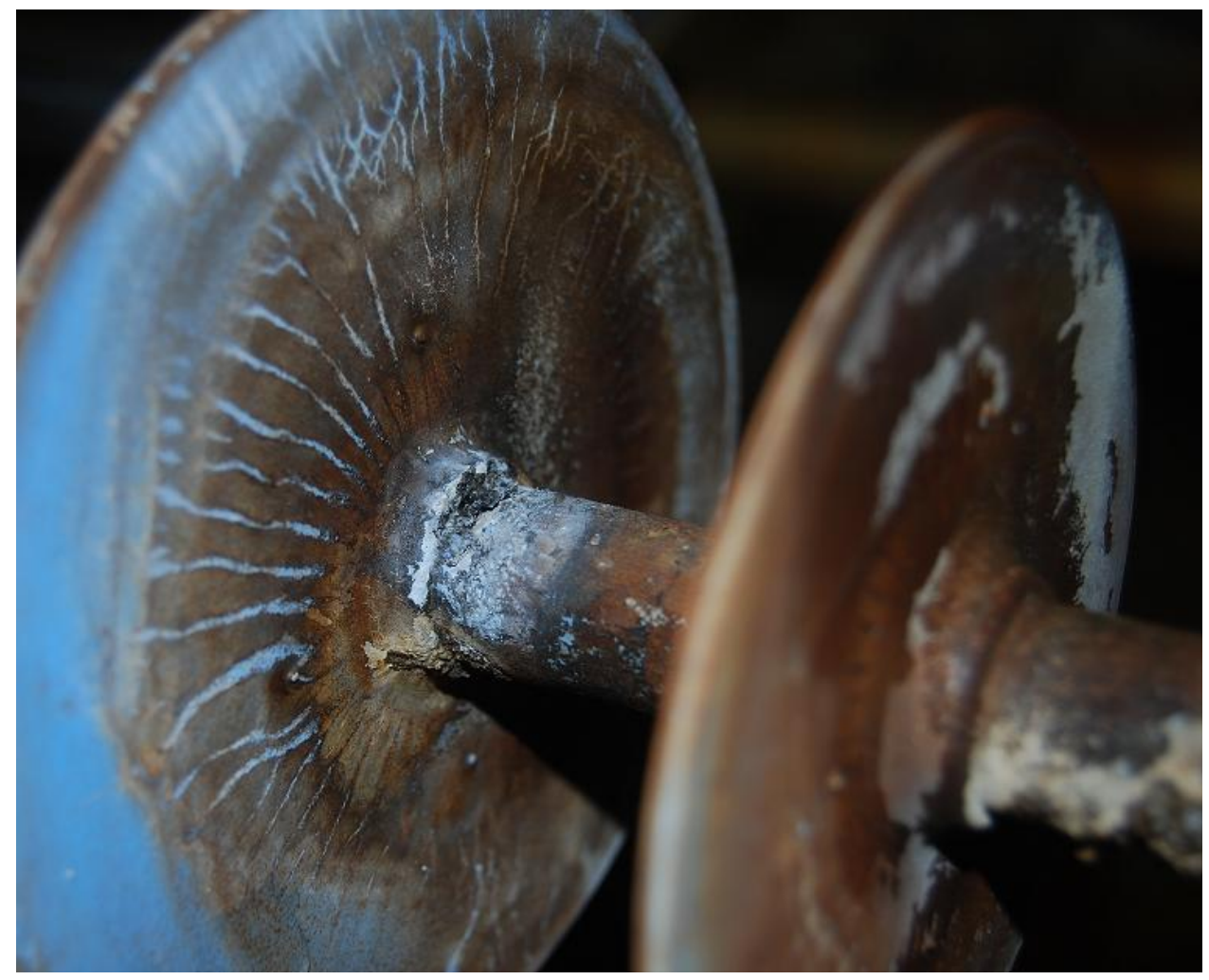

Figure 4.80: Detailed view of aging of the HTV SR insulator with a SCD of $35 \mathrm{~mm} / \mathrm{kV}$ for negative polarity HVDC excitation

Figure 4.81 compares the HTV SR insulators with a unified $S_{C D}$ of $28 \mathrm{~mm} / \mathrm{kV}$ (Channel 3) and a unified SCDof $35 \mathrm{~mm} / \mathrm{kV}$ (Channel 4). Channel 3 seems to have aged worse when compared to Channel 4 , since Channel 3 has a very severe black burn while Channel 4 does not have any burn on the rods and sheds. Both Channel 3 and Channel 4 have tracks in the form of a star effect on the pollution layer. Channel 3 has about four punctures, while Channel 4 only has two punctures. Both Channel 3 and Channel 4 have a black/brownish discoloration.
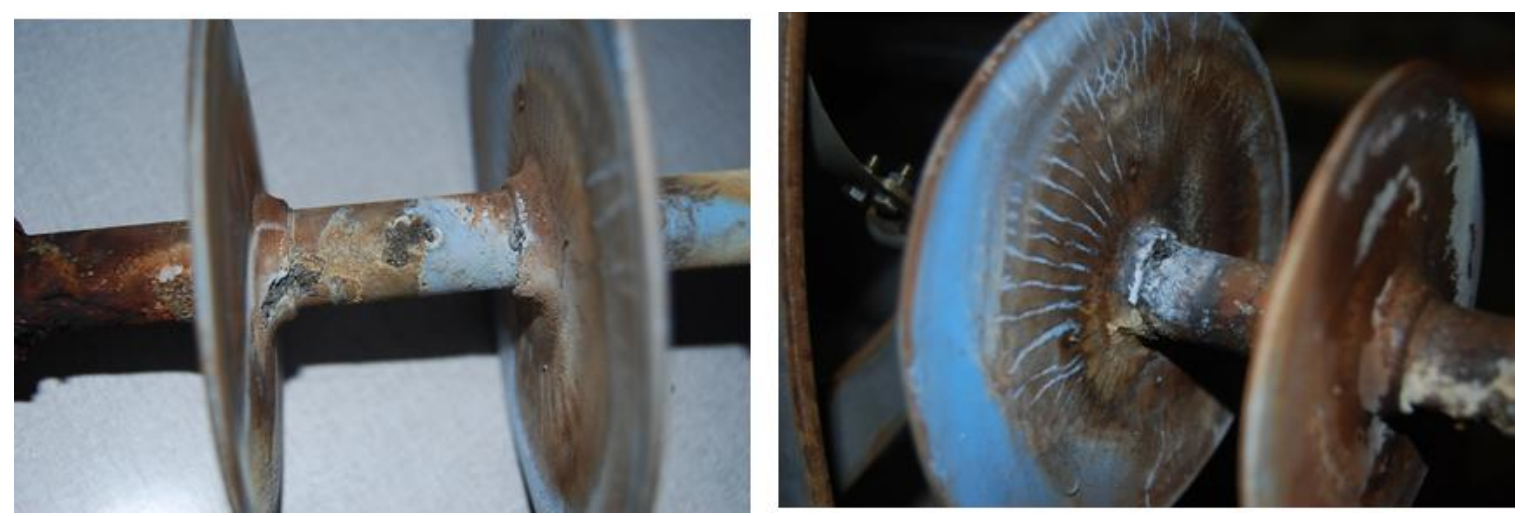

Figure 4.81: Channel 3 and Channel 4 after the test with negative polarity HVDC excitation 
Figure 4.82 shows the porcelain insulator (Channel 5) before and after the test, while Figure 4.83 shows the aging in more detail. The insulator did not age much and there is only minor brownish discoloration on the insulator.
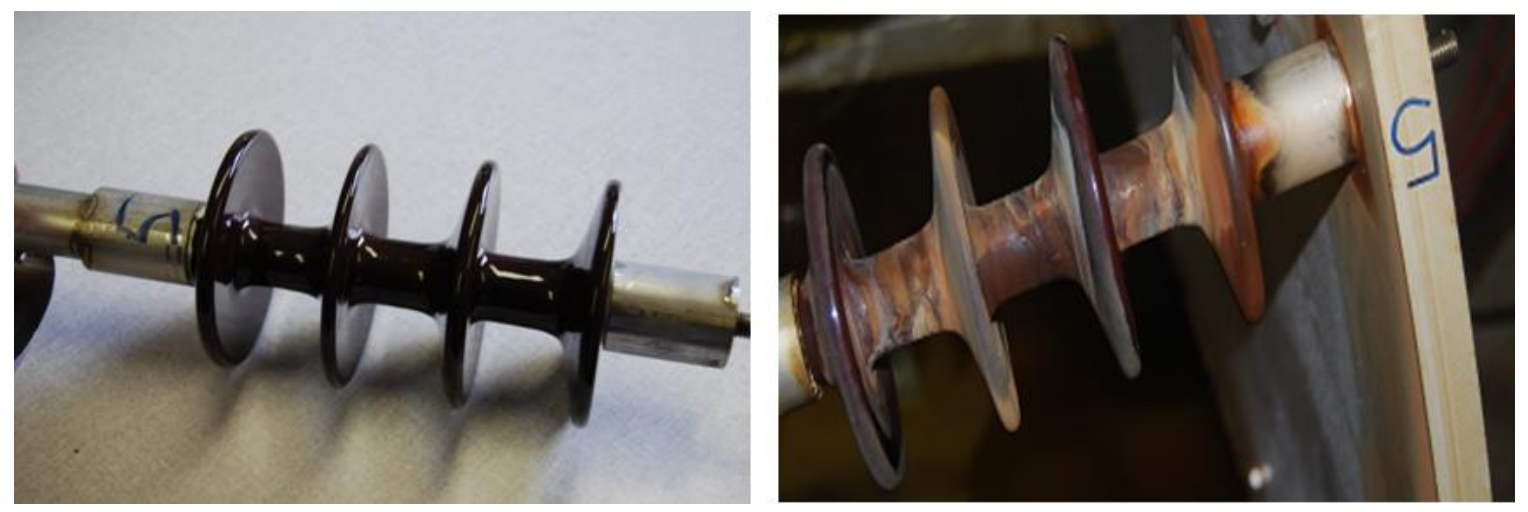

Figure 4.82: The porcelain insulator (Channel 5) before and after the test with negative polarity HVDC excitation

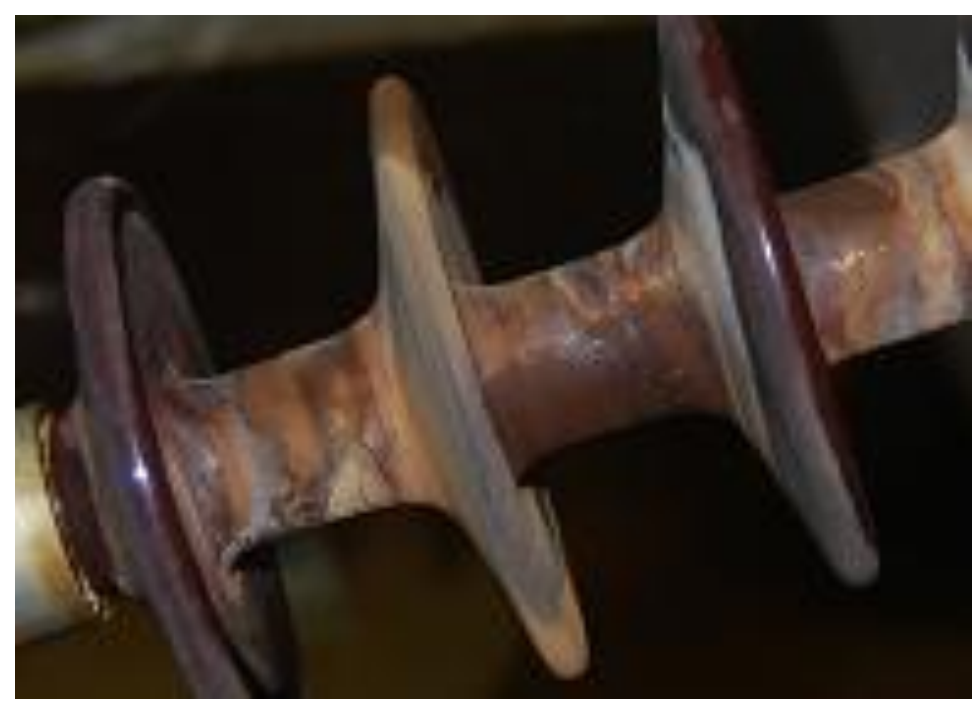

Figure 4.83: Detailed view of aging of the porcelain insulator for negative polarity HVDC excitation

Figure 4.84 shows the RTV SR coated porcelain insulator (Channel 6) before and after the test, while Figure 4.85 shows the aging in more detail. The insulator has both severe tracking and erosion and a brownish/reddish discoloration. Some of the material has flaked off. 

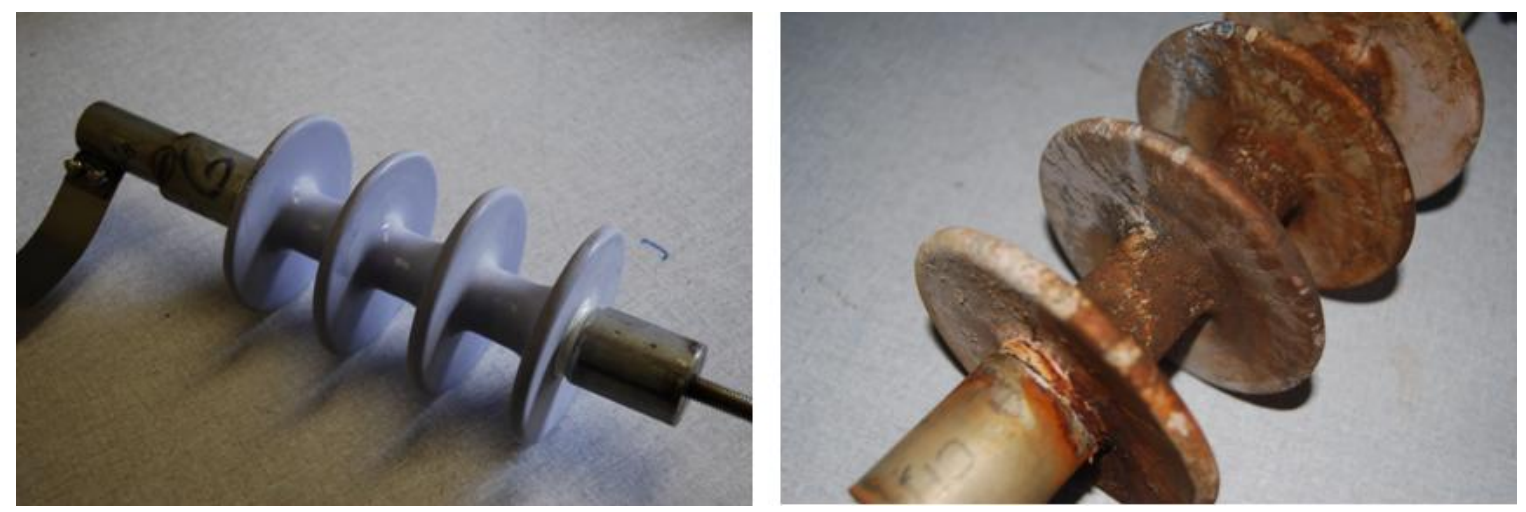

Figure 4.84: The RTV SR coated porcelain insulator (Channel 6) before and after the test with negative polarity HVDC excitation

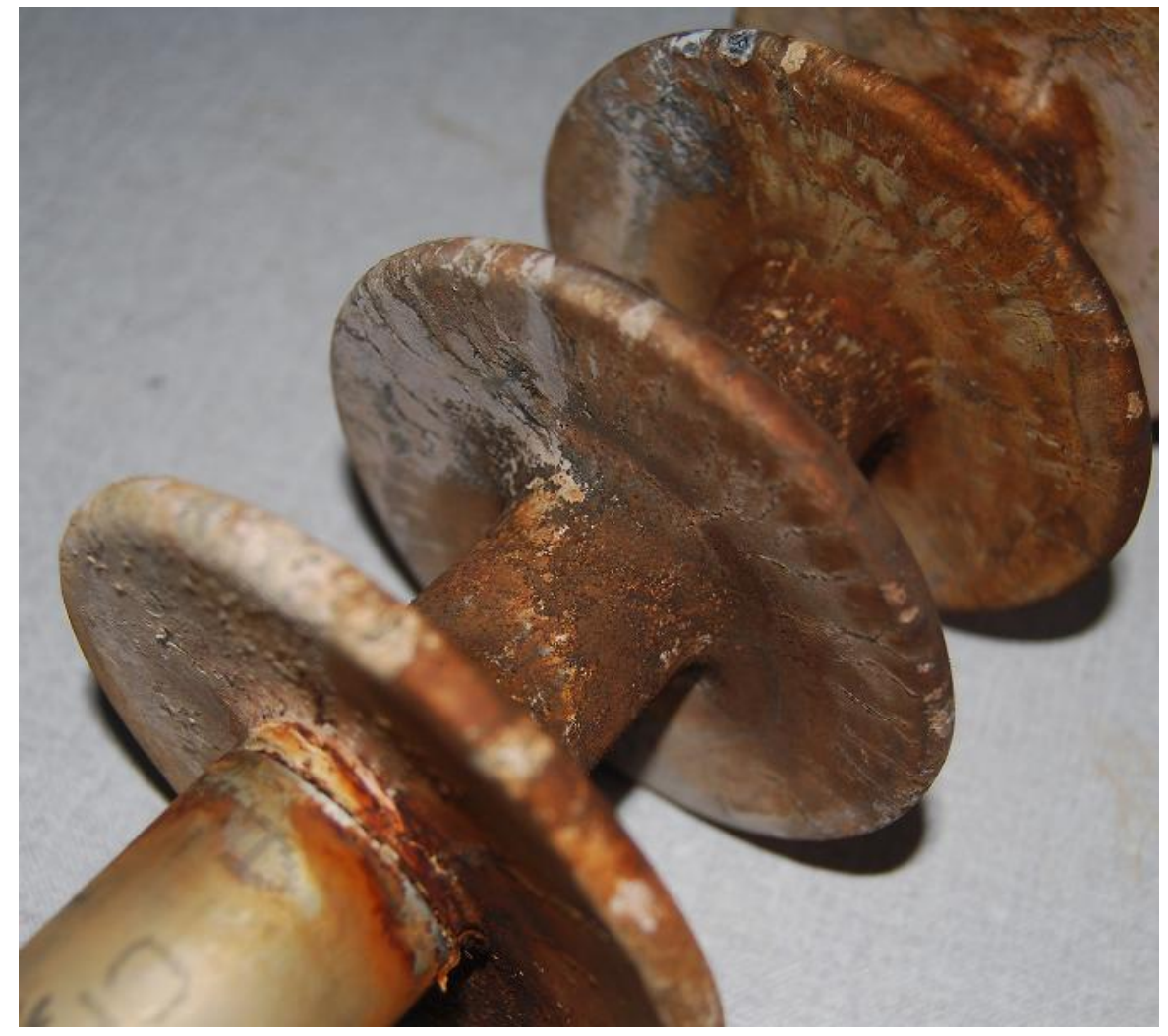

Figure 4.85: Detailed view of aging of the RTV coated porcelain insulator for negative polarity HVDC excitation

Figure 4.86 compares the reference porcelain insulator (Channel 5) and the RTV SR coated porcelain insulator (Channel 6). The porcelain insulator did not age much, while RTV SR coated porcelain insulator aged severely, with severe tracking and erosion. The porcelain insulator has a brown discoloration, while the RTV SR coated porcelain insulator has a brown/reddish discoloration. 

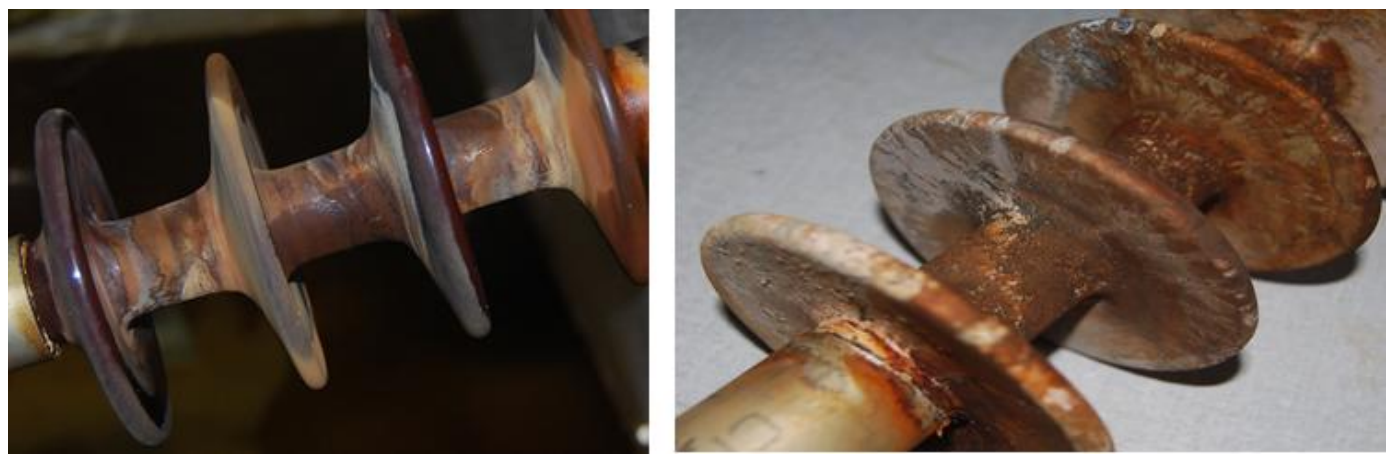

Figure 4.86: Channel 5 and Channel 6 after the test with negative polarity HVDC excitation

\subsubsection{Hydrophobic properties}

Figure 4.87 compares the hydrophobicity of the EPDM insulator (Channel 1) and the HTV SR insulator (Channel 2). The EPDM insulator as well as the HTV SR insulator has a wettability class of 5 . Thus, both insulators were becoming almost hydrophilic.
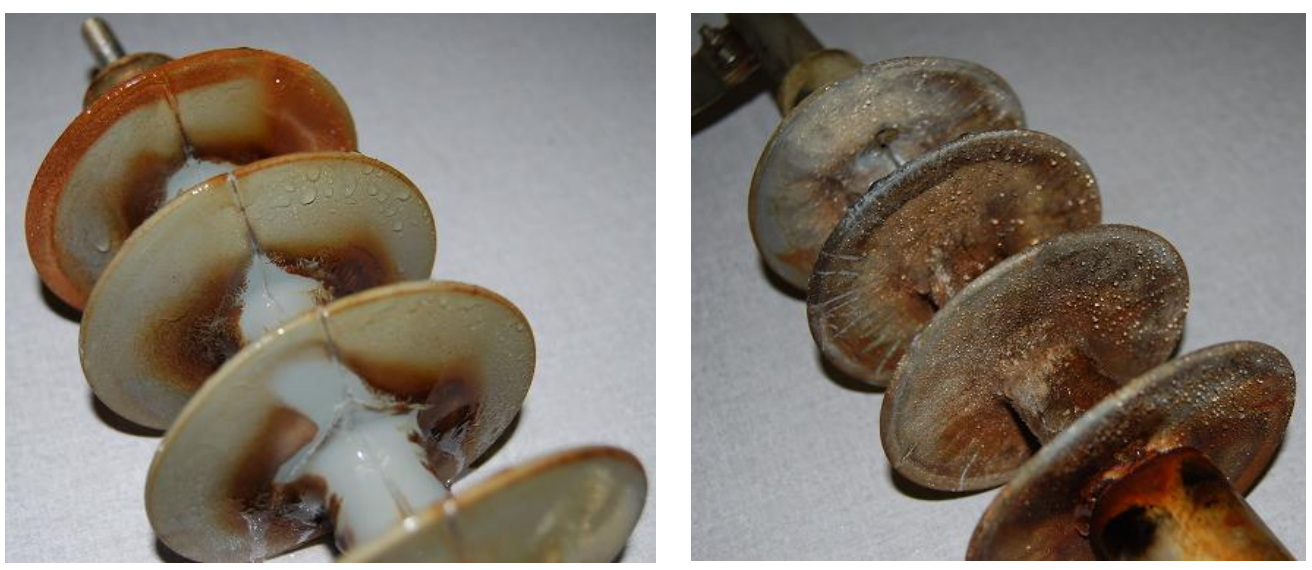

Figure 4.87: Wettability of the EPDM insulator (Channel 1) and the HTV SR insulator (Channel 2), both with a unified $S_{C D}$ of $28 \mathrm{~mm} / \mathrm{kV}$

Figure 4.88 compares the hydrophobicity of the HTV SR insulators with a unified SCDof $28 \mathrm{~mm} / \mathrm{kV}$ from manufacturer A (Channel 2) and from manufacturer B (Channel 3). Channel 2 has a wettability class of 5, while Channel 3 had a wettability class of 4 . 

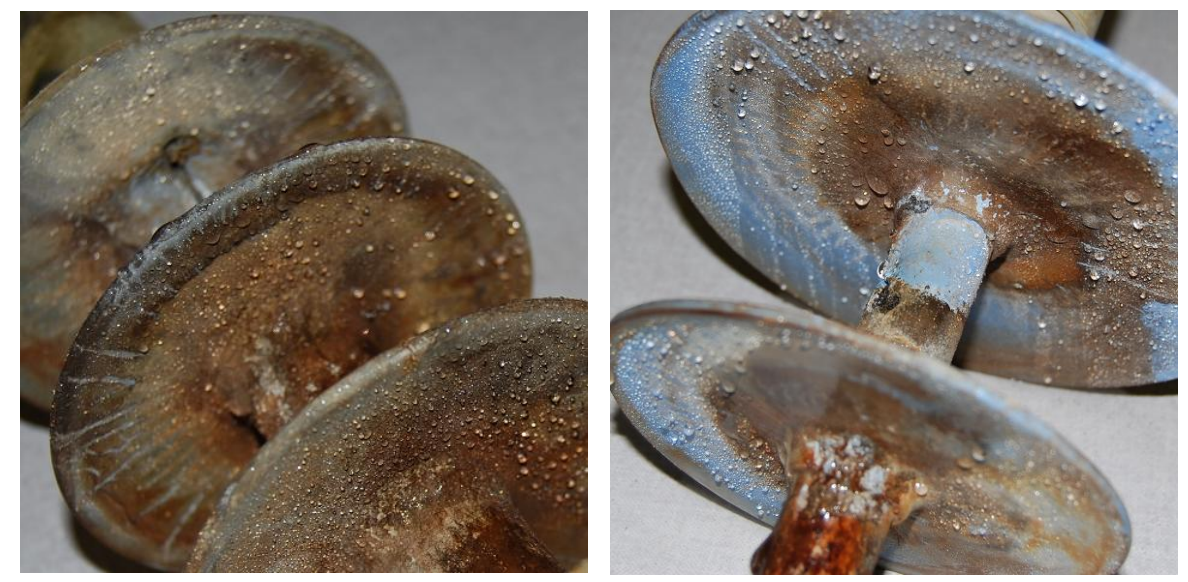

Figure 4.88: Wettability of the HTV SR insulators with a unified SCD of $28 \mathrm{~mm} / \mathrm{kV}$ from manufacturer A (Channel 2) and from manufacturer B (Channel 3)

Figure 4.89 compares the hydrophobicity of the HTV SR insulator with a unified SCDof $28 \mathrm{~mm} / \mathrm{kV}$ (Channel 3) and the HTV SR insulator with a unified SCDof 35 $\mathrm{mm} / \mathrm{kV}$ (Channel 4). Channel 3 has a wettability class of 4 and Channel 4 has a wettability class of 5 . Thus, Channel 3 seems to retain hydrophobicity better compared to Channel 4.
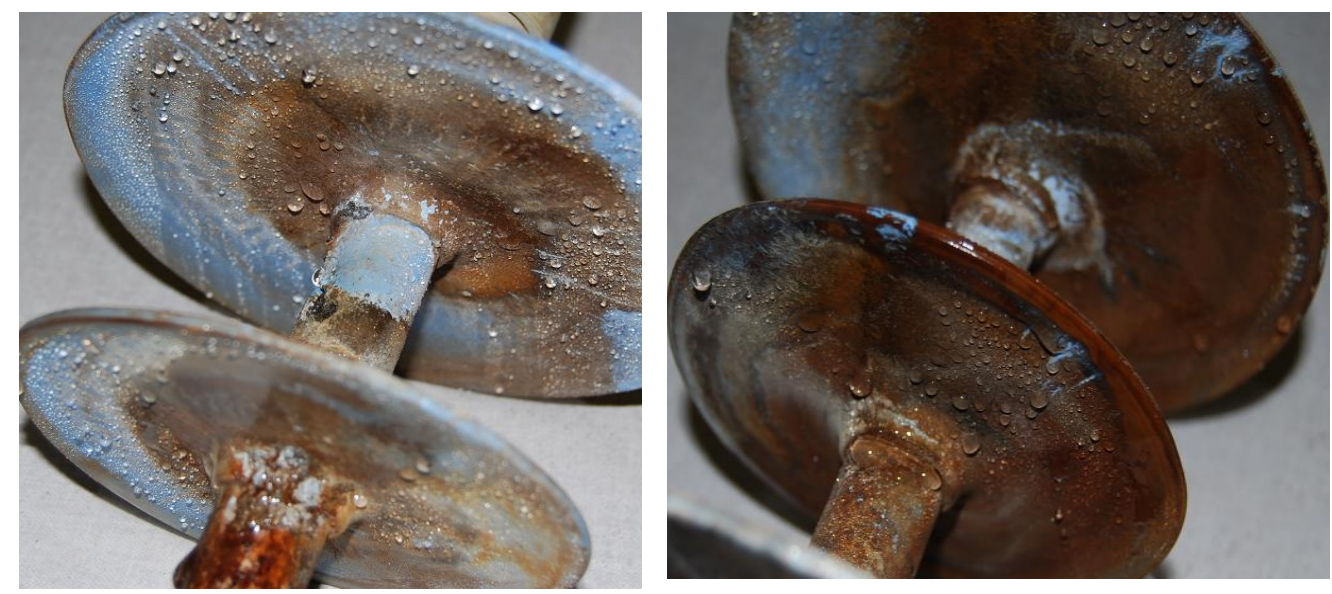

Figure 4.89: Wettability of the HTV SR insulators with a unified SCD of $28 \mathrm{~mm} / \mathrm{kV}$ (Channel 3) and a unified SCD of $35 \mathrm{~mm} / \mathrm{kV}$ (Channel 4)

Figure 4.90 compares the hydrophobicity of the porcelain insulator (Channel 5) and the RTV SR coated porcelain insulator (Channel 6). The reference porcelain insulator has a wettability class of 6 and is completely hydrophilic. The RTV SR coated porcelain insulator has a wettability class of 4 and still retains hydrophobicity. Thus, the coating does improve the hydrophobicity or wetting class of the insulators. 

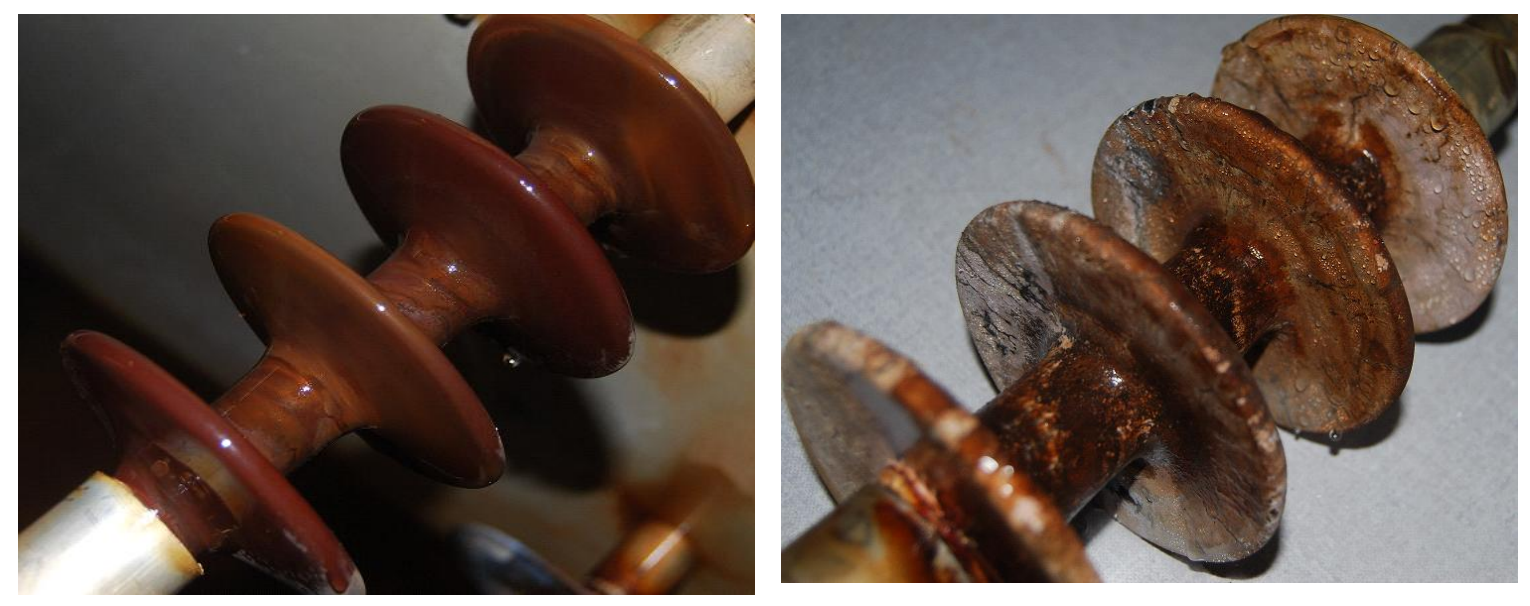

Figure 4.90: Wettability of porcelain insulator (Channel 5) and the RTV SR coated porcelain insulator (Channel 6) 


\section{Comparison of Results for Different Excitation Types}

\subsection{Overview}

The aging results for the different insulator samples of series two for HVAC and positive and negative polarity HVDC excitation is to be compared. The objective of the comparison is to determine how the type of excitation affected the aging performance of similar samples. The criteria addressed in the comparison are visual degradation, peak current and hydrophobicity.

\subsubsection{Results obtained for the EPDM insulator (channel 1) with a unified specific creepage distance of $28 \mathrm{~mm} / \mathrm{kV}$ from manufacturer $\mathrm{A}$}

Figure 5.1shows the material aging of the EPDM insulator (Channel 1) for HVAC and positive and negative polarity HVDC excitation. The EPDM insulator under HVAC excitation had discoloration, widening on the mould lines, and little brown bubbles on the mould lines. The EPDM insulator under positive HVDC excitation had discoloration and severe erosion localised to the mould lines. The EPDM insulator under negative HVDC excitation had less erosion along the mould lines, with sharp erosion on the sheds along the mould lines. The diagram shows that the EPDM aged the worst for the positive polarity HVDC excitation, followed by the negative HVDC excitation and finally the HVAC excitation.
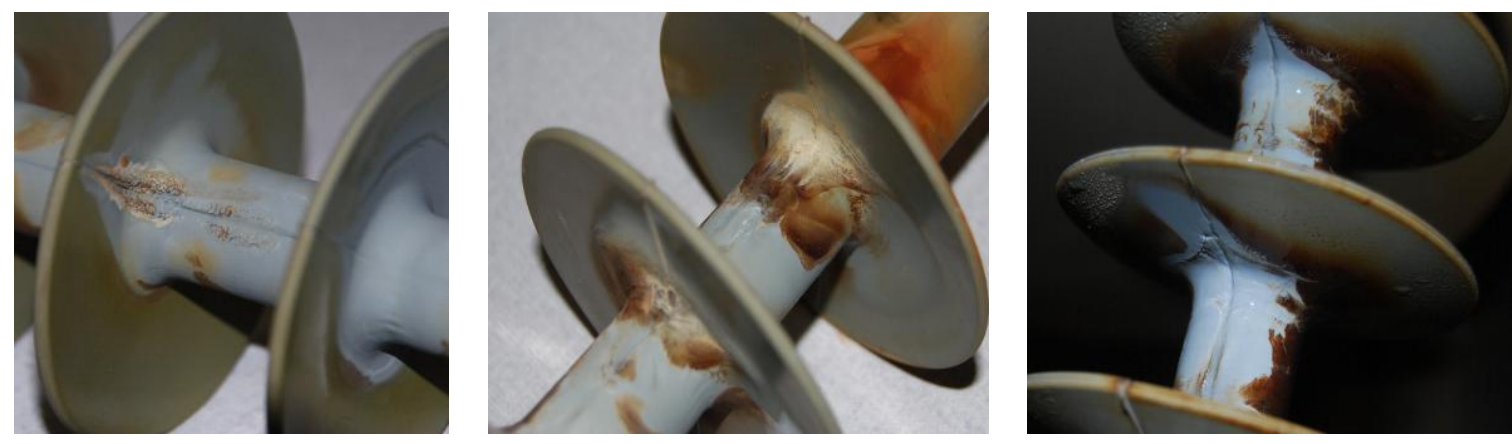

Figure 5.1: Comparison of material aging of the EPDM insulator for HVAC and positive and negative polarity HVDC excitation

Figure 5.2, Figure 5.3 and Figure 5.4 shows the peak currents for the EPDM insulator (channel 1) for HVAC and positive and negative polarity HVDC excitation respectively. Figure 5.2 shows the positive and negative peak currents for the EPDM insulators for HVAC excitation. The initial maximum peak current is $45 \mathrm{~mA}$, whereas the maximum peak current was $55 \mathrm{~mA}$ when the test was stopped after 54 days. Figure 5.3 shows the peak currents for the EPDM insulator (channel 1) for 
positive polarity HVDC excitation. Initially the maximum positive peak current was $35 \mathrm{~mA}$, whereas the maximum peak current was $78 \mathrm{~mA}$ when the test was stopped after 48 days. This experiment ran for a total of 41 days. Figure 5.4 shows the peak currents for the EPDM insulator (channel 1) for negative polarity HVDC excitation. Initially the maximum negative peak current was $20 \mathrm{~mA}$, whereas the maximum peak current was $55 \mathrm{~mA}$ when the test was stopped after 43 days. This experiment ran for approximately 41 days.

Table 5.1: Peak currents for the EPDM insulator (channel 1) for HVAC and positive and negative polarity HVDC excitation

\begin{tabular}{|l|l|l|l|}
\hline Insulator type & $\begin{array}{l}\text { EPDM insulator } \\
\text { (channel 1); HVAC } \\
\text { excitation }\end{array}$ & $\begin{array}{l}\text { EPDM insulator } \\
\text { (channel 1); positive } \\
\text { HVDC excitation }\end{array}$ & $\begin{array}{l}\text { EPDM insulator } \\
\text { (channel 1); negative } \\
\text { HVDC excitation }\end{array}$ \\
\hline $\begin{array}{l}\text { Peak current [mA] in } \\
\text { the beginning }\end{array}$ & $45 \mathrm{~mA}$ & $20 \mathrm{~mA}$ \\
\hline $\begin{array}{l}\text { Peak current [mA] in } \\
\text { the end }\end{array}$ & $55 \mathrm{~mA}$ & $78 \mathrm{~mA}$ & $55 \mathrm{~mA}$ \\
\hline $\begin{array}{l}\text { Hydrophobicity lost } \\
\text { Duration of the } \\
\text { experiment }\end{array}$ & $\begin{array}{l}\text { Immediately after test } \\
\text { voltage is applied. }\end{array}$ & $\begin{array}{l}\text { Immediately after test } \\
\text { voltage is applied. }\end{array}$ & $\begin{array}{l}\text { Immediately after test } \\
\text { voltage is applied. }\end{array}$ \\
\hline
\end{tabular}

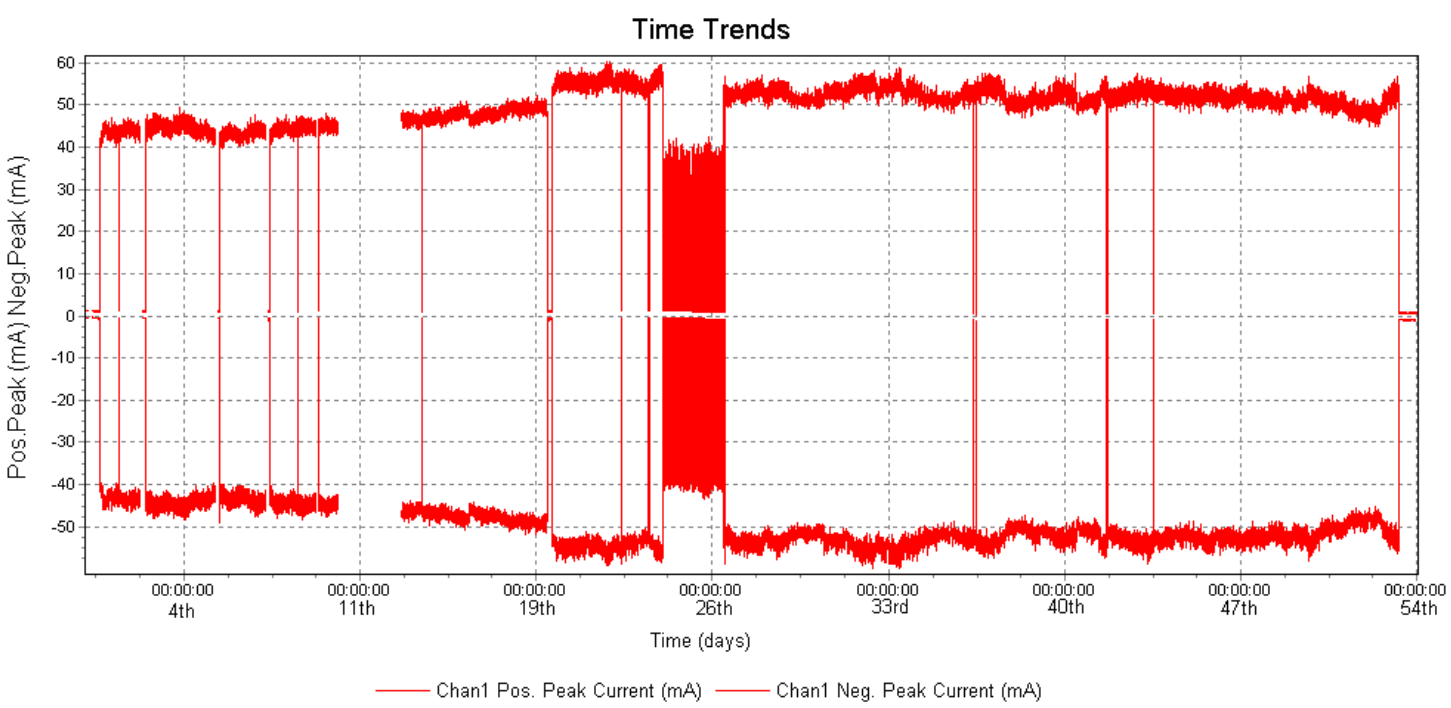

Figure 5.2: Peak currents for the EPDM insulator (channel 1) for HVAC excitation 


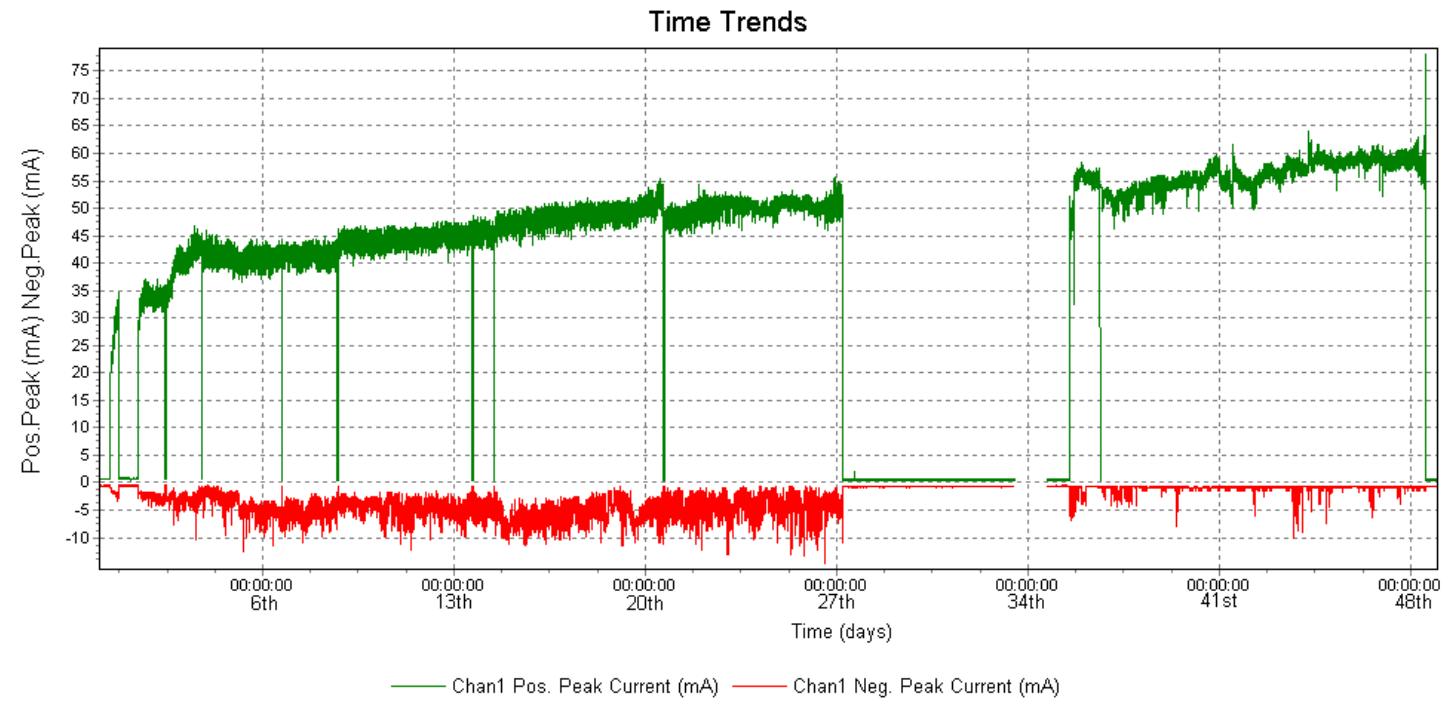

Figure 5.3: Peak currents for the EPDM insulator (channel 1) for positive polarityHVDC excitation.

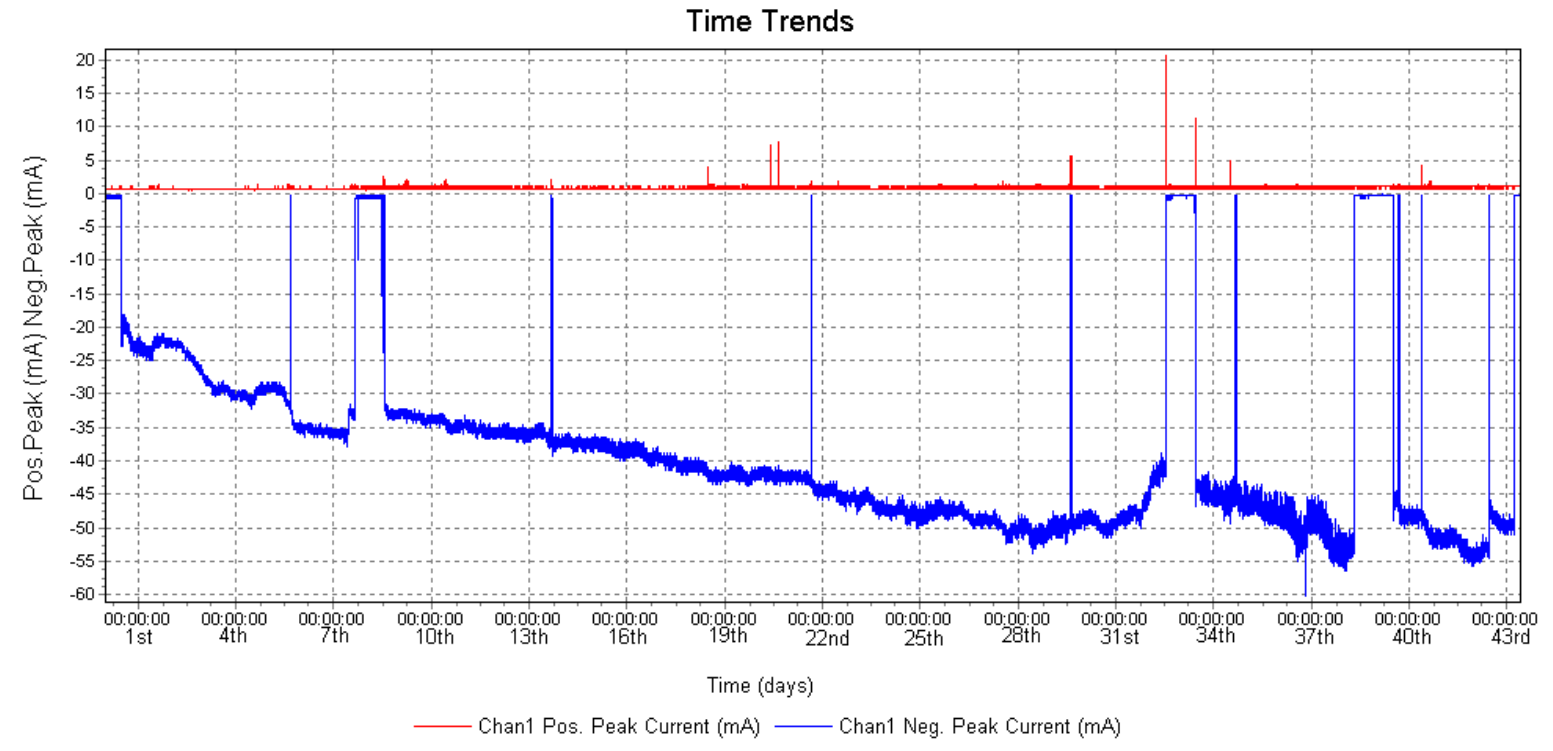

Figure 5.4: Peak currents for the EPDM insulator (channel 1) for negative HVDC excitation

Figure 5.5 shows the hydrophobicity of the EPDM insulator (channel 1) for HVAC and positive and negative polarity HVDC excitation. The EPDM insulator fell within a wettability class of 5 with the HVAC and positive and negative polarity HVDC excitation. The EPDM insulator is almost hydrophilic and did not recover its hydrophobicity for any of the excitation types. 

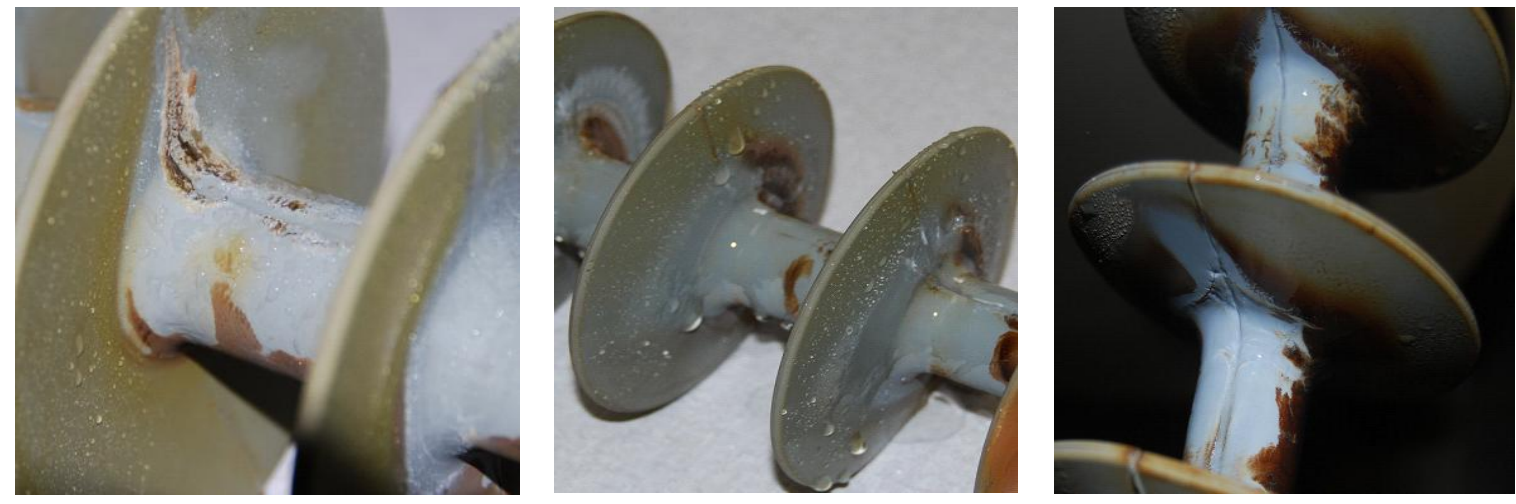

Figure 5.5: Comparison of hydrophobicity for the EPDM insulator (channel 1) for HVAC and positive and negative polarity HVDC excitation

\subsubsection{Results obtained for the HTV SR insulator (channel 2) with a unified specific creepage distance of $28 \mathrm{~mm} / \mathrm{kV}$ from manufacturer $A$}

Figure 5.6 shows the HTV SR insulator (channel 2) for HVAC and positive and negative polarity HVDC excitation. The HTV SR insulator aged the slowest for HVAC excitation, as it showed only a brown discoloration as well as light tracking on the surface. The HTV SR insulator aged severely for positive polarity HVDC excitation. It had severe tracks and erosion in the form of a star shape. This insulator showed heavy discoloration, flaking of material, as well as a puncture. The HTV SR insulator had aged faster for negative polarity HVDC excitation than for HVAC excitation, but not as severely as for the positive polarity HVDC excitation. The HTV SR insulator for negative polarity HVDC excitation also showed brown discoloration, severe erosion and tracks in the form of the star. The HTV SR insulator, therefore, had aged most severely for positive polarity HVDC excitation, followed by the negative polarity excitation and had aged very slowly for HVAC excitation.
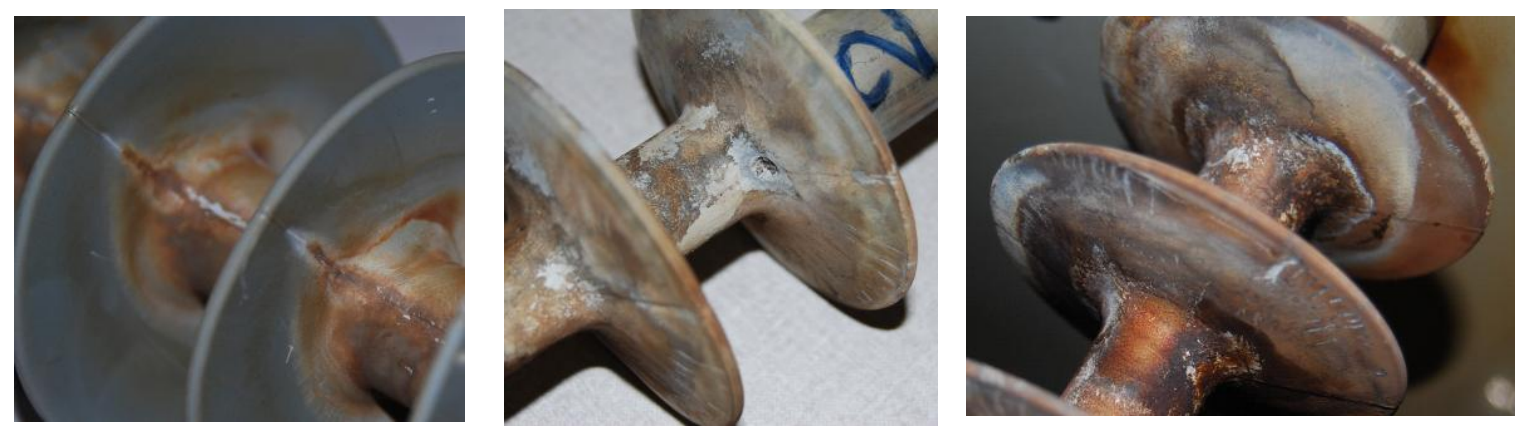

Figure 5.6: Comparison of aging for the HTV SR insulator (channel 2) for HVAC and positive and negative polarity HVDC excitation

Figure 5.7 shows the peak currents for HVAC excitation. Initially the maximum peak current was $40 \mathrm{~mA}$, whereas the maximum peak current was $60 \mathrm{~mA}$ when the test was stopped on the $53^{\text {rd }}$ day. Figure 5.8 shows the peak currents for the HTV SR insulator 
(channel 2) for positive polarity HVDC excitation. Initially the maximum positive peak current was $30 \mathrm{~mA}$, whereas the maximum peak current was $151 \mathrm{~mA}$ when the test was stopped. Figure 5.9 shows the peak currents for the HTV SR insulator for negative HVDC excitation. Initially the maximum negative peak current was $20 \mathrm{~mA}$, whereas the maximum peak current was $110 \mathrm{~mA}$ when the test was stopped on the $43^{\text {rd }}$ day.

Table 5.2: Peak currents for the HTV SR insulator (channel 2) for HVAC and positive and negative polarity HVDC excitation

\begin{tabular}{|l|l|l|l|}
\hline Insulator type & $\begin{array}{l}\text { HTV SR insulator } \\
\text { (channel 2) with } \\
\text { HVAC excitation }\end{array}$ & $\begin{array}{l}\text { HTV SR insulator } \\
\text { (channel 2) with } \\
\text { positive HVDC } \\
\text { excitation }\end{array}$ & $\begin{array}{l}\text { HTV SR insulator } \\
\text { (channel 2) with } \\
\text { negative HVDC } \\
\text { excitation }\end{array}$ \\
\hline $\begin{array}{l}\text { Peak current [mA] in } \\
\text { the beginning }\end{array}$ & $40 \mathrm{~mA}$ & $20 \mathrm{~mA}$ \\
\hline $\begin{array}{l}\text { Peak current [mA] in } \\
\text { the end }\end{array}$ & $60 \mathrm{~mA}$ & $151 \mathrm{~mA}$ & $110 \mathrm{~mA}$ \\
\hline $\begin{array}{l}\text { Hydrophobicity lost } \\
\text { Duration of the } \\
\text { experiment for } \\
\text { insulator }\end{array}$ & $\begin{array}{l}\text { Immediately after test } \\
\text { voltage is applied. }\end{array}$ & $\begin{array}{l}\text { Immediately after test } \\
\text { voltage is applied. }\end{array}$ & $\begin{array}{l}\text { Immediately after test } \\
\text { voltage is applied. }\end{array}$ \\
\hline
\end{tabular}

Initially, the HTV SR insulator had the highest peak current for HVAC excitation, followed by positive polarity HVDC excitation and then negative polarity HVDC excitation. At the end of the experiment, the HTV SR insulator with positive polarity HVDC excitation showed the highest peak current, followed by the HTV SR insulator with negative polarity HVDC excitation and then the HVAC excitation.

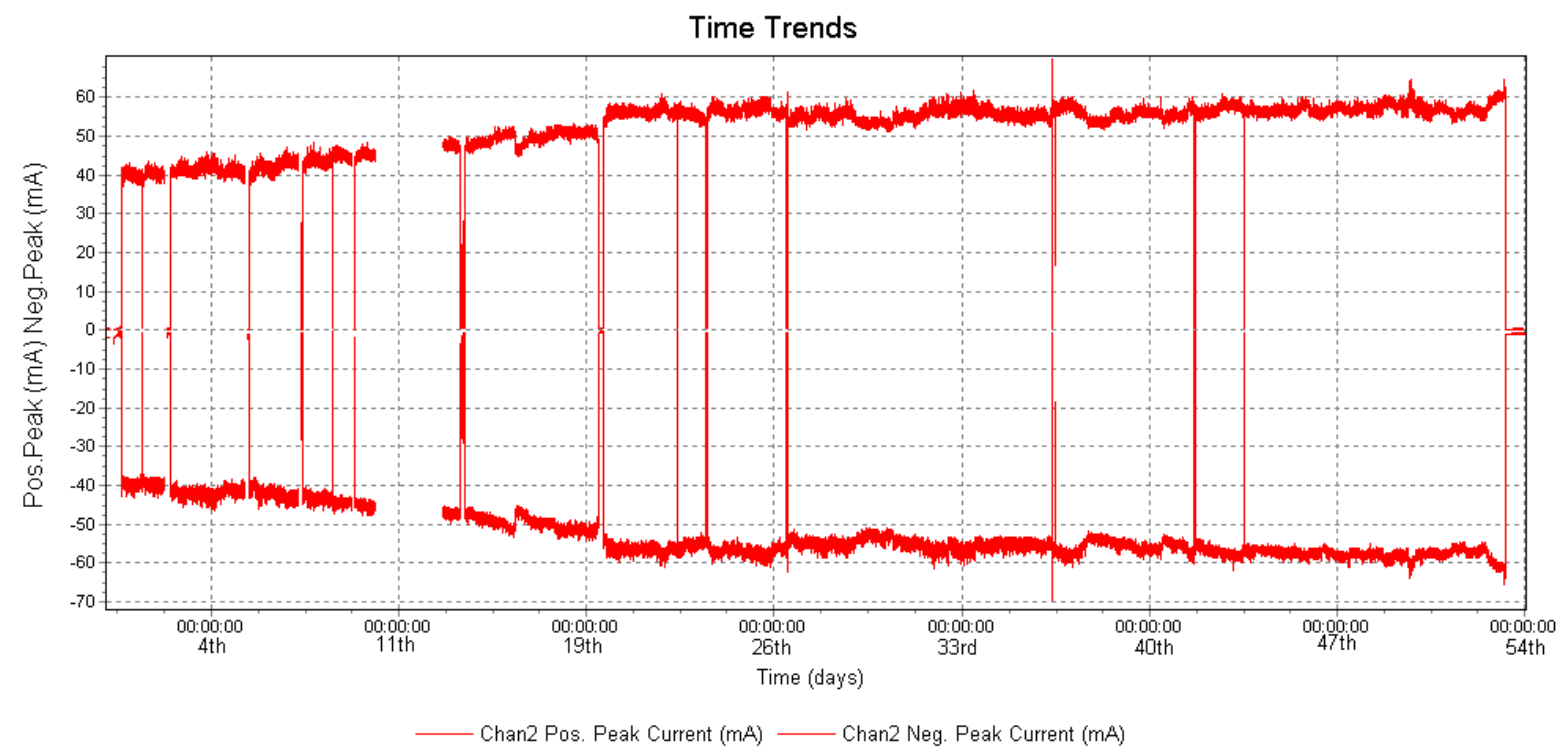

Figure 5.7: Peak currents for the HTV SR insulator (channel 2) for HVAC excitation 


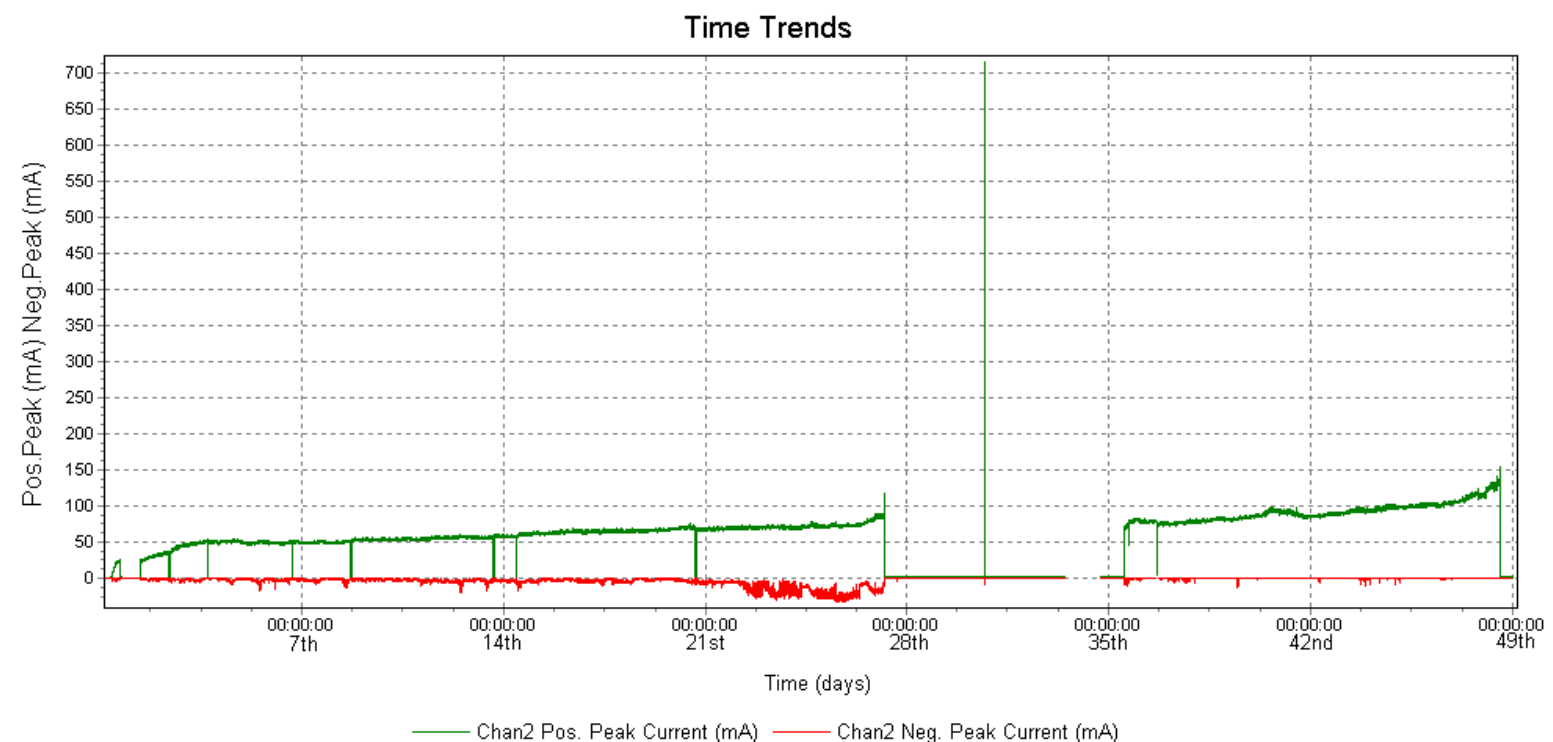

Figure 5.8: Peak currents for the HTV SR insulator (channel 2) for positive polarity HVDC excitation

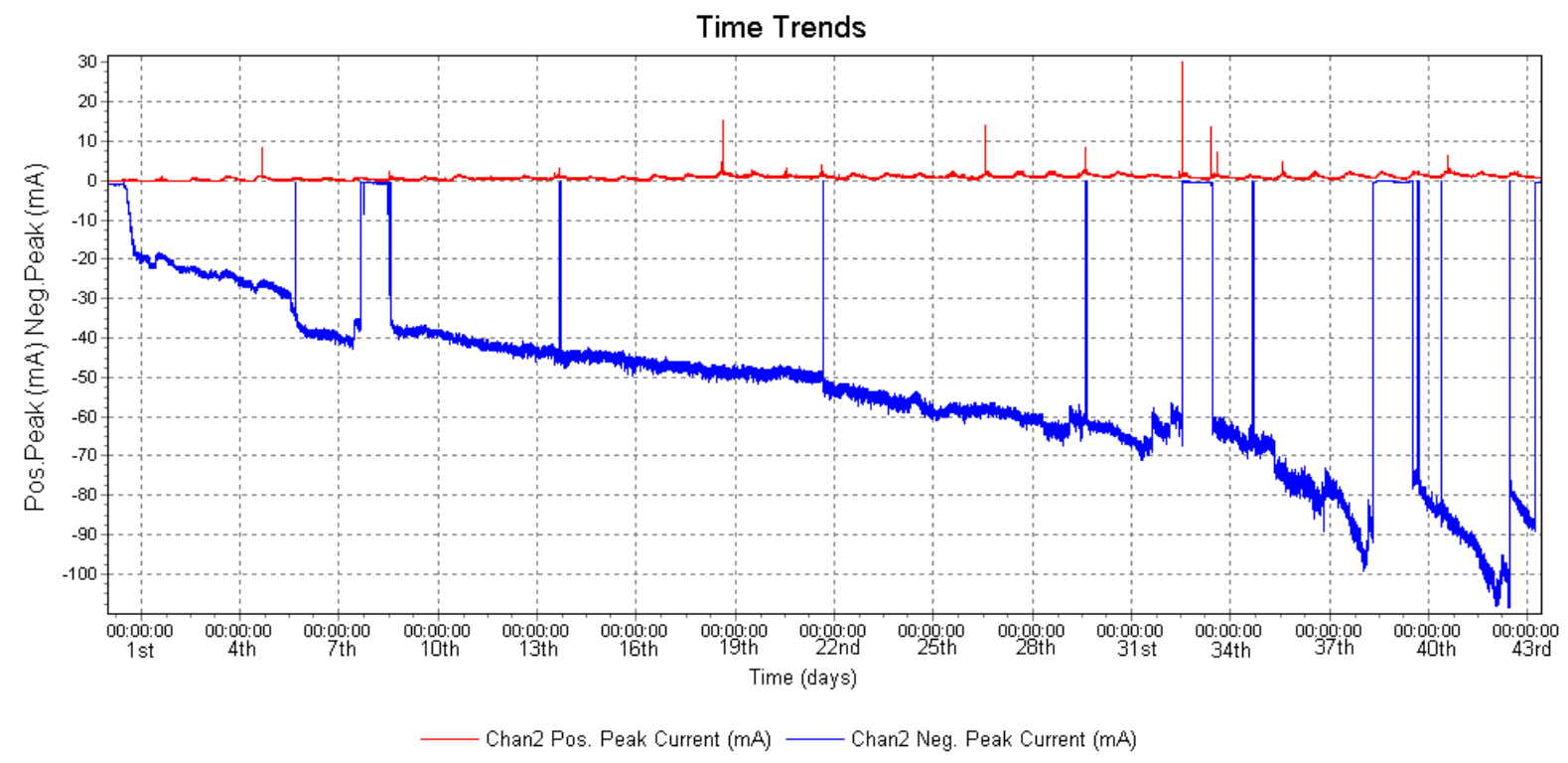

Figure 5.9: Peak currents for the HTV SR insulator (channel 2) for negative polarity HVDC excitation

Figure 5.10 shows the hydrophobicity for the HTV SR insulator (channel 2) of manufacturer A for HVAC and positive and negative polarity HVDC excitation. The HTV SR insulator (channel 2) fell within a wettability class of 1 for HVAC excitation, thus having recovered its hydrophovicity. The HTV SR insulator (channel 2) had a wettability class of 3 for positive polarity HVDC excitation excitation, implying that the insulator was still slightly hydrophobic. The HTV SR insulator had a wettability 
class of 5 for negative polarity HVDC excitation, showing that the material did not recover and became almost completely hydrophilic.
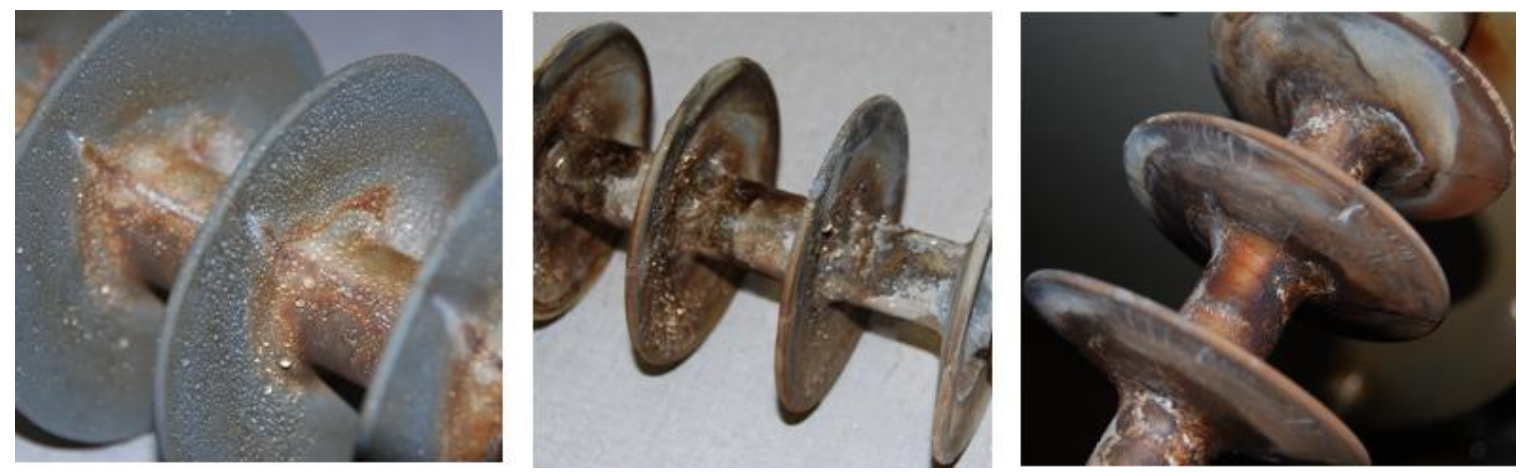

Figure 5.10: Comparison of the the hydrophobicity of the HTV SR insulator (channel 2) for HVAC and positive and negative polarity HVDC excitation

\subsubsection{Results obtained for the HTV SR insulator (channel 3) with a unified specific creepage distance of $28 \mathrm{~mm} / \mathrm{kV}$ from manufacturer $B$}

Figure 5.11 compares the material aging of the HTV SR insulator (channel 3) with unified SCDof $28[\mathrm{~mm} / \mathrm{kV}]$ from manufacturer B for HVAC excitation with that of the positive and negative polarity HVDC excitation respectively. The HTV SR insulator aged severely for HVAC excitation, but not as severe as for the other two cases. The HTV SR insulator had eight punctures, discoloration and small tracks on the surface for HVAC excitation. The HTV SR insulator showed some material and part of the shed removed from the insulator for positive polarity HVDC excitation. The HTV SR insulator had a part of the rod burned, turning it black, in colour for negative HVDC excitation. The HTV SR insulator with unified SCDof 28 [mm/kV] from manufacturer B performed the worst for positive HVDC excitation, followed by negative polarity HVDC excitation and performed the best with HVAC excitation.
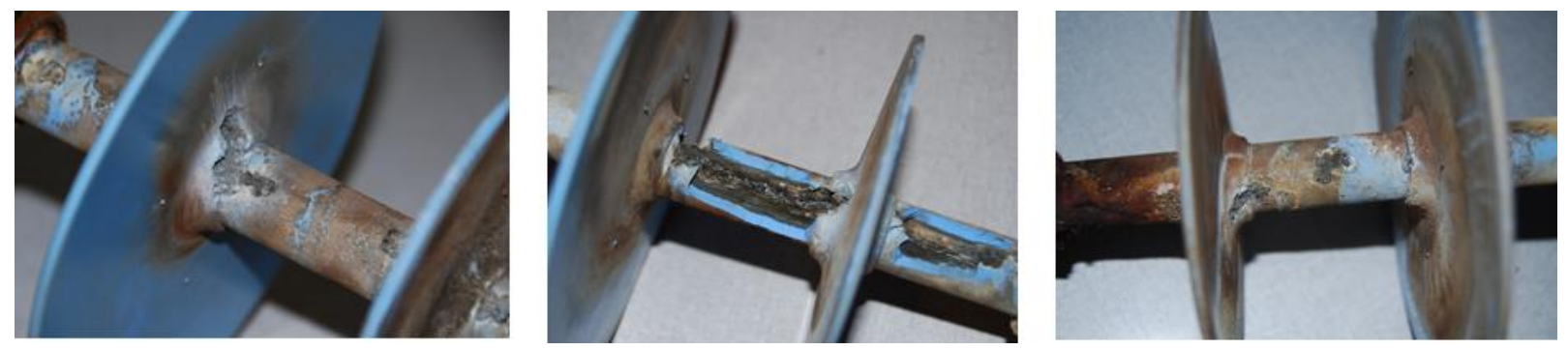

Figure 5.11: Comparison of aging for the HTV SR insulator (channel 3) with a unified SCD of $28 \mathrm{~mm} / \mathrm{kV}$ for HVAC and positive and negative polarity HVDC excitation

Figure 5.12 shows the initial peak current for HVAC excitation to be $40 \mathrm{~mA}$, with the peak current when the insulator failed on the $53^{\text {rd }}$ day equal to $450 \mathrm{~mA}$. Figure 5.13 shows the initial peak current for positive polarity HVDC excitation to be $30 \mathrm{~mA}$, 
with the peak current when the insulator failed on the $25^{\text {th }}$ day equal to $725 \mathrm{~mA}$. Figure 5.14 shows the initial peak current for negative polarity HVDC excitation to be $20 \mathrm{~mA}$, with the peak current when the insulator failed on the $38^{\text {th }}$ day equal to 544 $\mathrm{mA}$.

Initially, the HTV SR insulator (channel 3) had a lower peak current for negative HVDC excitation, followed by positive polarity HVDC excitation and the highest peak current for HVAC excitation. The HTV SR insulator (channel 3) took a very short time before the insulator tripped for positive polarity HVDC excitation, a longer time to trip for HVAC excitation and even longer for negative polarity HVDC excitation.

Table 5.3: Peak currents for the HTV SR insulator (channel 3) for HVAC and positive and negative polarity HVDC excitation

\begin{tabular}{|l|l|l|l|}
\hline Insulator type & $\begin{array}{l}\text { HTV SR insulator } \\
\text { (channel 3) with } \\
\text { HVAC excitation }\end{array}$ & $\begin{array}{l}\text { HTV SR insulator } \\
\text { (channel 3) with } \\
\text { positive HVDC } \\
\text { excitation }\end{array}$ & $\begin{array}{l}\text { HTV SR insulator } \\
\text { (channel 3) with } \\
\text { negative HVDC } \\
\text { excitation }\end{array}$ \\
\hline $\begin{array}{l}\text { Peak current [mA] in } \\
\text { the beginning }\end{array}$ & $40 \mathrm{~mA}$ & $20 \mathrm{~mA}$ \\
\hline $\begin{array}{l}\text { Peak current [mA] in } \\
\text { the end }\end{array}$ & $\begin{array}{l}450 \mathrm{~mA} \\
\text { "tripped" }\end{array}$ & $\begin{array}{l}724 \mathrm{~mA} \\
\text { "tripped" }\end{array}$ & $\begin{array}{l}544 \mathrm{~mA} \\
\text { "tripped" }\end{array}$ \\
\hline $\begin{array}{l}\text { Hydrophobicity lost } \\
\text { Immediately after test } \\
\text { voltage is applied. }\end{array}$ & $2 \mathrm{~h} 30$ minutes later & 3 days \\
\hline $\begin{array}{l}\text { Duration of the } \\
\text { experiment }\end{array}$ & 52 days & 38 days \\
\hline
\end{tabular}

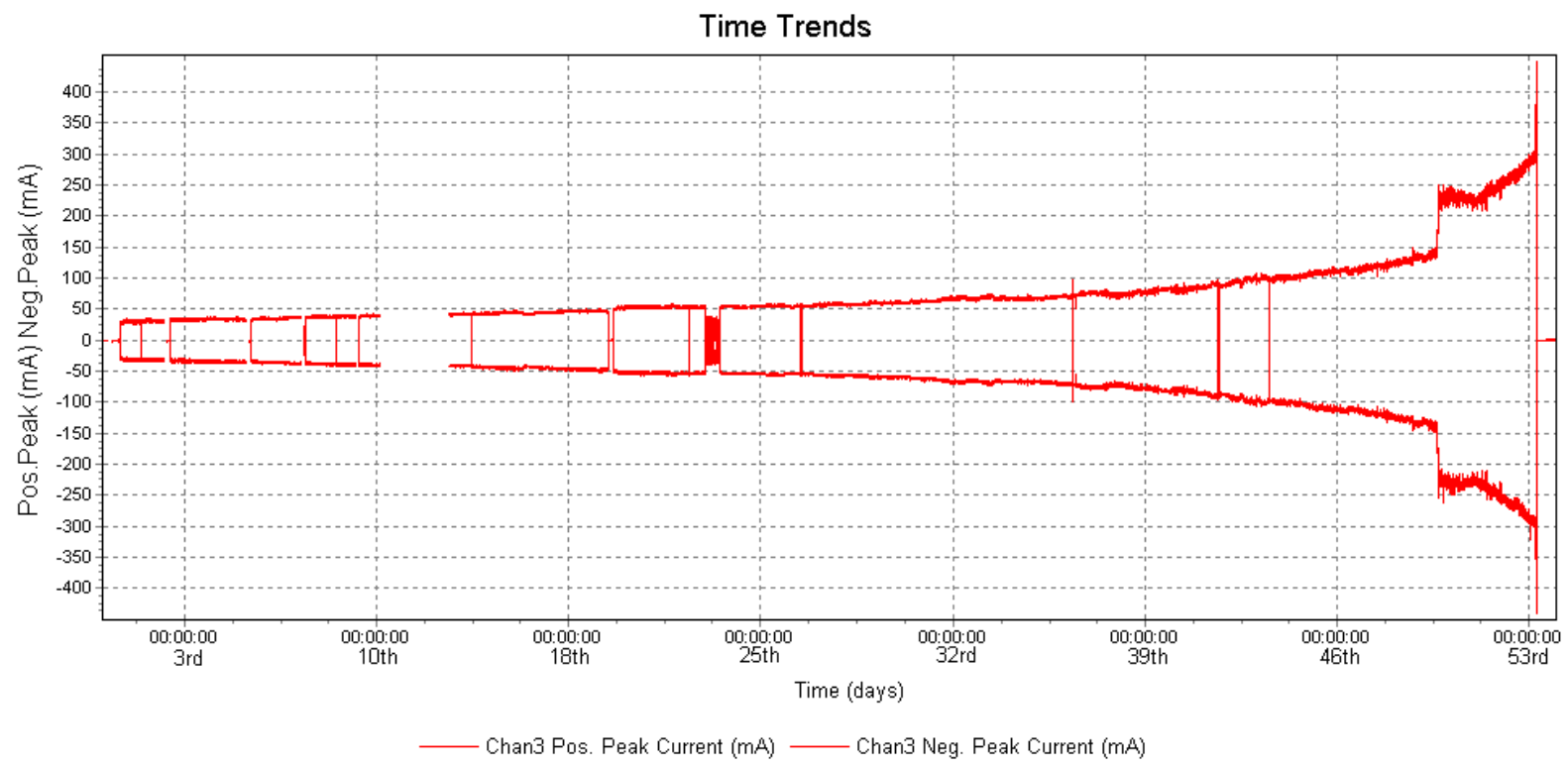


Figure 5.12: Peak currents for HTV SR insulator (channel 3) with a unified SCD of 28 $\mathrm{mm} / \mathrm{kV}$ for HVAC excitation

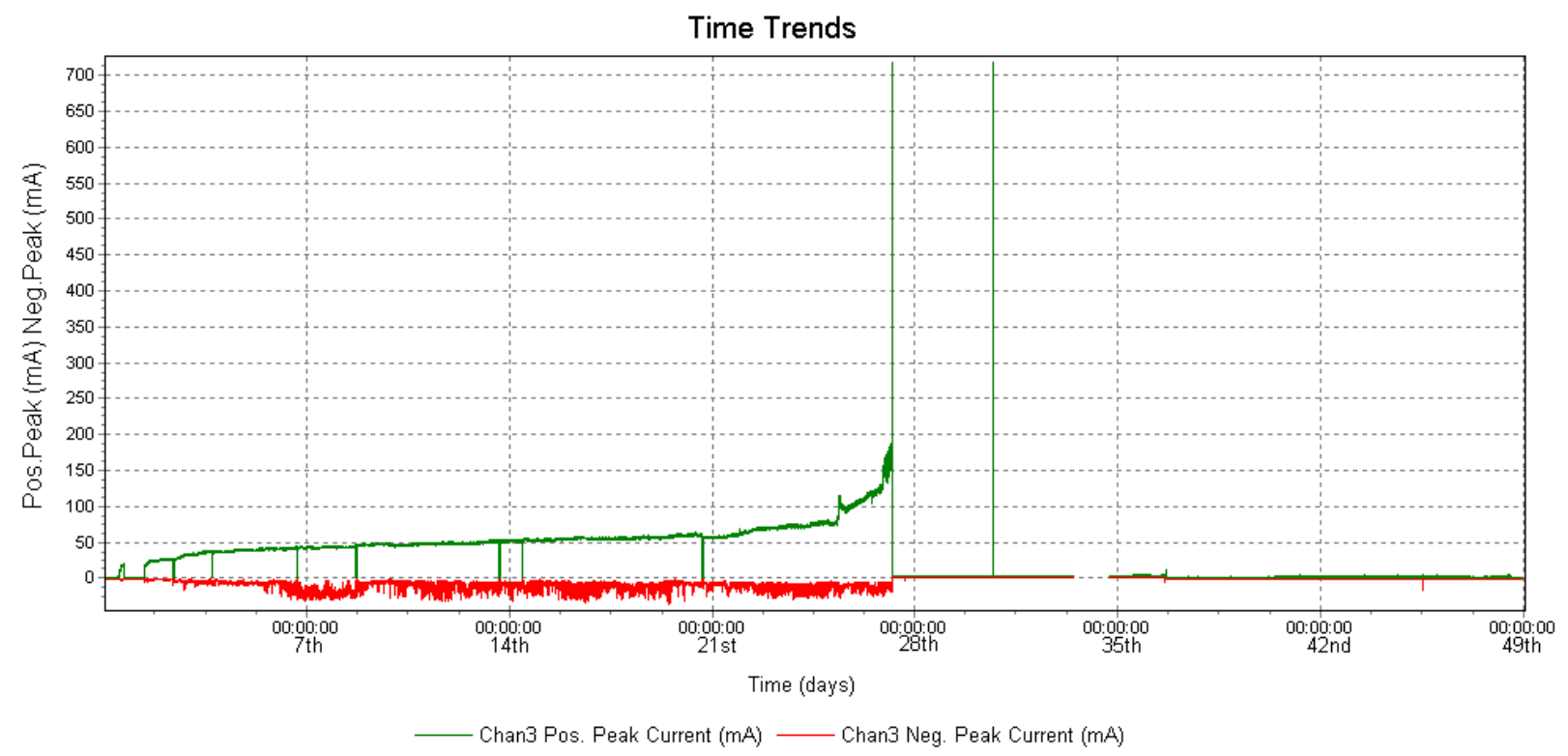

Figure 5.13: Peak currents for HTV SR insulator (channel 3) with a unified SCD of 28 $\mathrm{mm} / \mathrm{kV}$ for positive polarity HVDC excitation

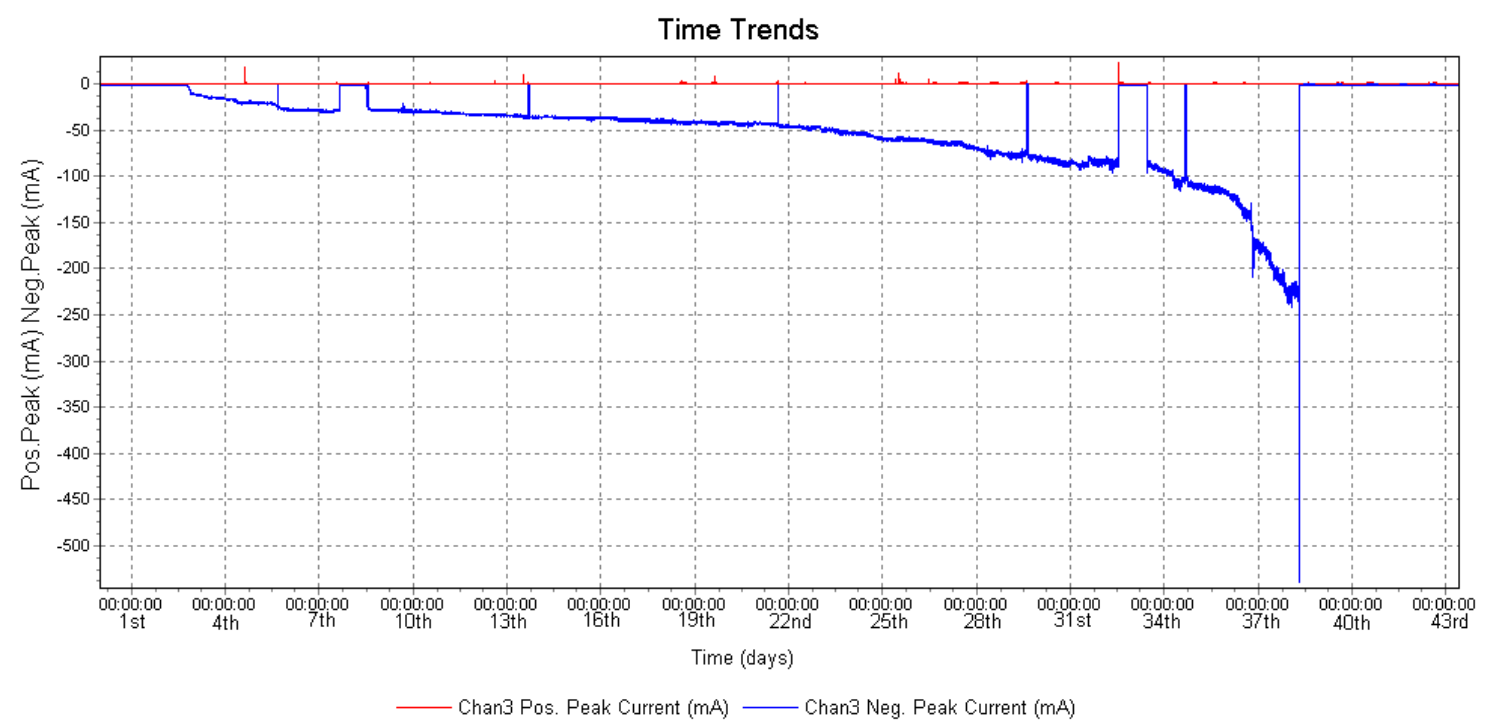

Figure 5.14: Peak currents for HTV SR insulator (channel 3) with a unified SCD of 28 $\mathrm{mm} / \mathrm{kV}$ for negative polarity HVDC excitation

Figure 5.15 compares the hydrophobicity of the HTV SR insulator (channel 3) for HVAC and positive and negative polarity HVDC excitation. The HTV SR insulator fell within a wettability class of 2 for HVAC excitation, a wettability class of 3 for positive polarity HVDC excitation and a wettability class of 4 for negative polarity HVDC excitation. The HTV SR insulator seems to retain its hydrophobicity better for HVAC excitation, followed by positive and then negative polarity HVDC excitation. 

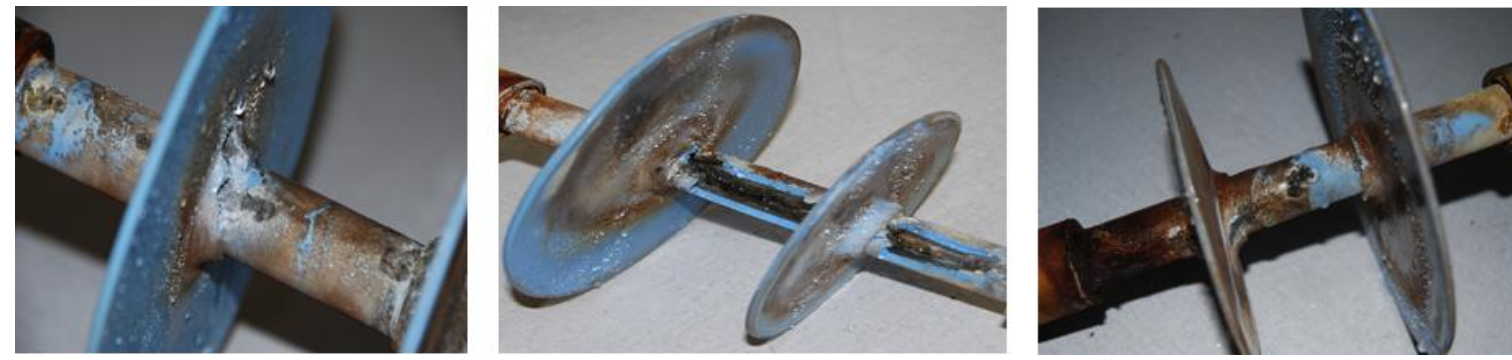

Figure 5.15: Comparison of hydrophobicity for the HTV SR insulator (channel 3) with a unified SCD of $28 \mathrm{~mm} / \mathrm{kV}$ for HVAC and positive and negative polarity HVDC excitation

\subsubsection{Results obtained for the HTV SR insulator (channel 4) with a unified specific creepage distance of $35 \mathrm{~mm} / \mathrm{kV}$ from manufacturer $B$}

Figure 5.16 compares the material aging of the HTV SR insulator (channel 3) with a unified SCDof $35 \mathrm{~mm} / \mathrm{kV}$ from manufacturer B for HVAC excitation with that for positive and negative polarity HVDC excitation respectively. The HTV SR insulator (channel 4) did not age significantly for HVAC excitation and showed only slight discoloration and light tracking on the surface. The HTV SR insulator (Channel 4) had degraded severely for positive polarity HVDC excitation. The insulator had a lot of punctures, tracks, erosion in the form of a star and was heavily discoloured. The HTV SR insulator had aged severely for negative polarity HVDC excitation. It had punctures, tracks on the surface and was heavily discoloured.

Upon comparison of the three cases, it was found that the HTV SR insulator (Channel 4) did not age significantly for HVAC excitation, but aged severely for HVDC excitation, with the positive polarity excitation showing the most severe signs of aging.
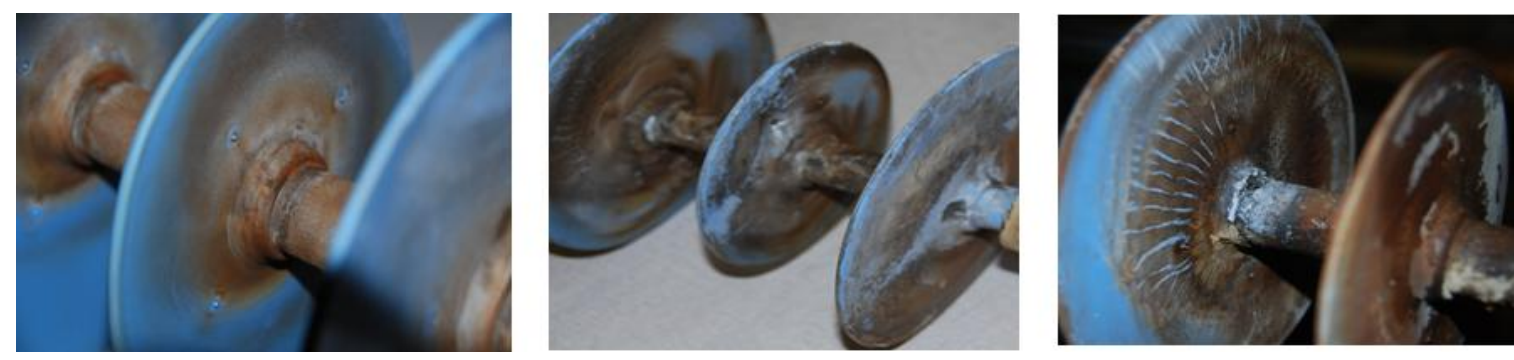

Figure 5.16: Comparison of material aging of the HTV SR insulator (channel 4) for HVAC and positive and negative polarity HVDC excitation

Figure 5.17 shows the initial peak current for HVAC excitation to be $25 \mathrm{~mA}$, with the peak current on the last $\left(53^{\text {rd }}\right)$ day of the test equal to $65 \mathrm{~mA}$. Figure 5.18 shows the initial peak current for positive HVDC excitation to be $20 \mathrm{~mA}$, with the peak current when the insulator failed on the $53^{\text {rd }}$ day equal to $420 \mathrm{~mA}$. Figure 5.19 shows the 
initial peak current for negative HVDC excitation to be $8 \mathrm{~mA}$, with the peak current on the last $\left(53^{\text {rd }}\right)$ day of the test equal to $75 \mathrm{~mA}$. Initially, the peak current for the HTV SR insulator (channel 4) was the highest for HVAC excitation followed by positive and then negative polarity HVDC excitation. The leakage current at the end of each test was the highest for positive polarity HVDC excitation, followed by negative polarity HVDC excitation and then HVAC excitation.

Table 5.4: Peak currents for the HTV SR insulator (channel 4) for HVAC and positive and negative polarity HVDC excitation

\begin{tabular}{|l|l|l|l|}
\hline Insulator type & $\begin{array}{l}\text { HTV SR insulator } \\
\text { (channel 4) with } \\
\text { HVAC excitation }\end{array}$ & $\begin{array}{l}\text { HTV SR insulator } \\
\text { (channel 4) with } \\
\text { positive HVDC } \\
\text { excitation }\end{array}$ & $\begin{array}{l}\text { HTV SR insulator } \\
\text { (channel 4) with } \\
\text { negative HVDC } \\
\text { excitation }\end{array}$ \\
\hline $\begin{array}{l}\text { Peak current [mA] in } \\
\text { the beginning }\end{array}$ & $25 \mathrm{~mA}$ & $20 \mathrm{~mA}$ & $20 \mathrm{~mA}$ \\
\hline $\begin{array}{l}\text { Peak current [mA] in } \\
\text { the end }\end{array}$ & $65 \mathrm{~mA}$ & $\begin{array}{l}420 \mathrm{~mA} \\
\text { "tripped" }\end{array}$ & $\begin{array}{l}700 \mathrm{~mA} \\
\text { "tripped" }\end{array}$ \\
\hline $\begin{array}{l}\text { Hydrophobicity lost } \\
\text { Immediately after test } \\
\text { voltage is applied. }\end{array}$ & $2 \mathrm{~h} 30$ minutes later & 3 days \\
\hline $\begin{array}{l}\text { Duration of the } \\
\text { experiment }\end{array}$ & 52 days & 41 days & 41 days \\
\hline
\end{tabular}

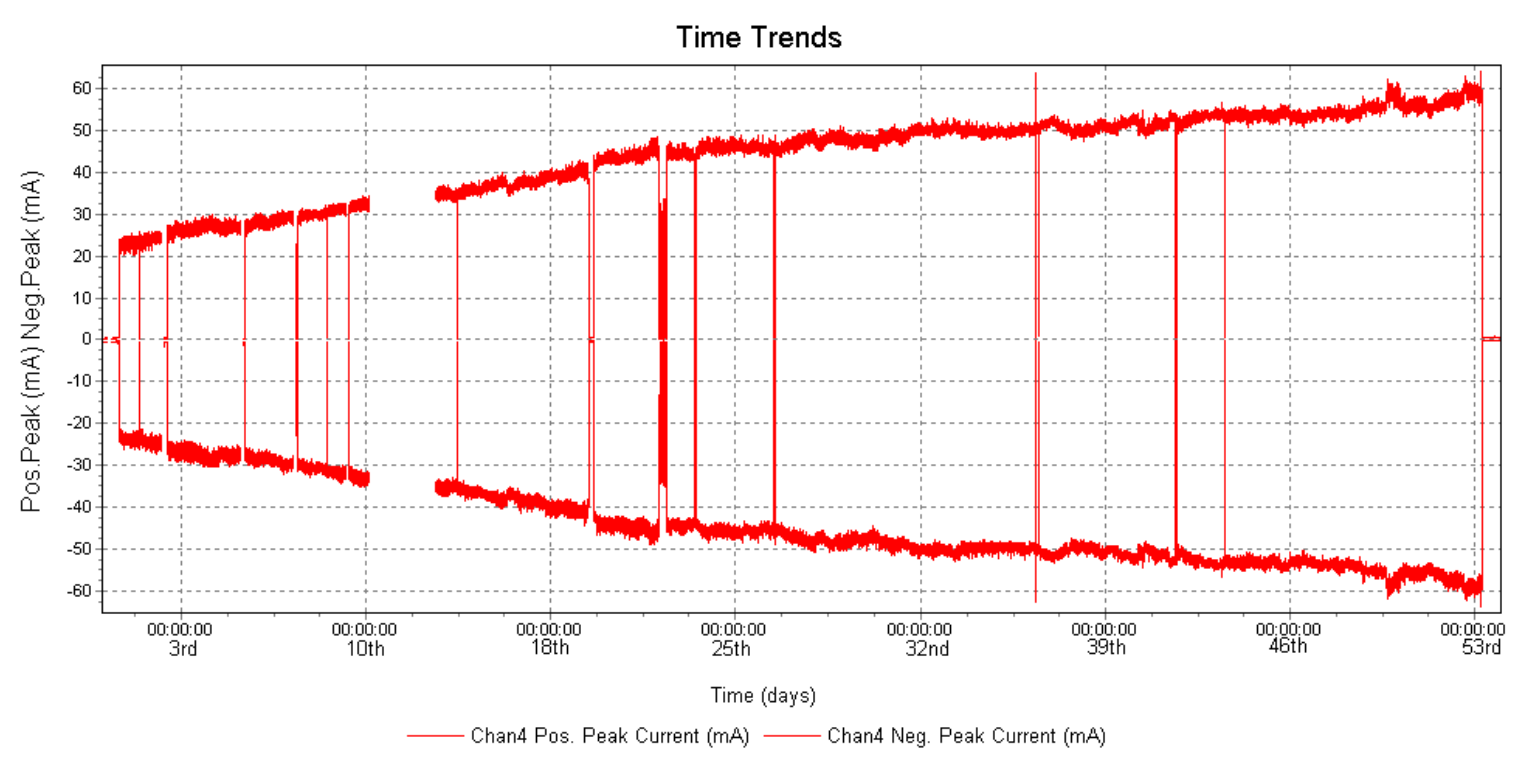

Figure 5.17: Peak current for the HTV SR insulator (channel 4) for HVAC excitation 


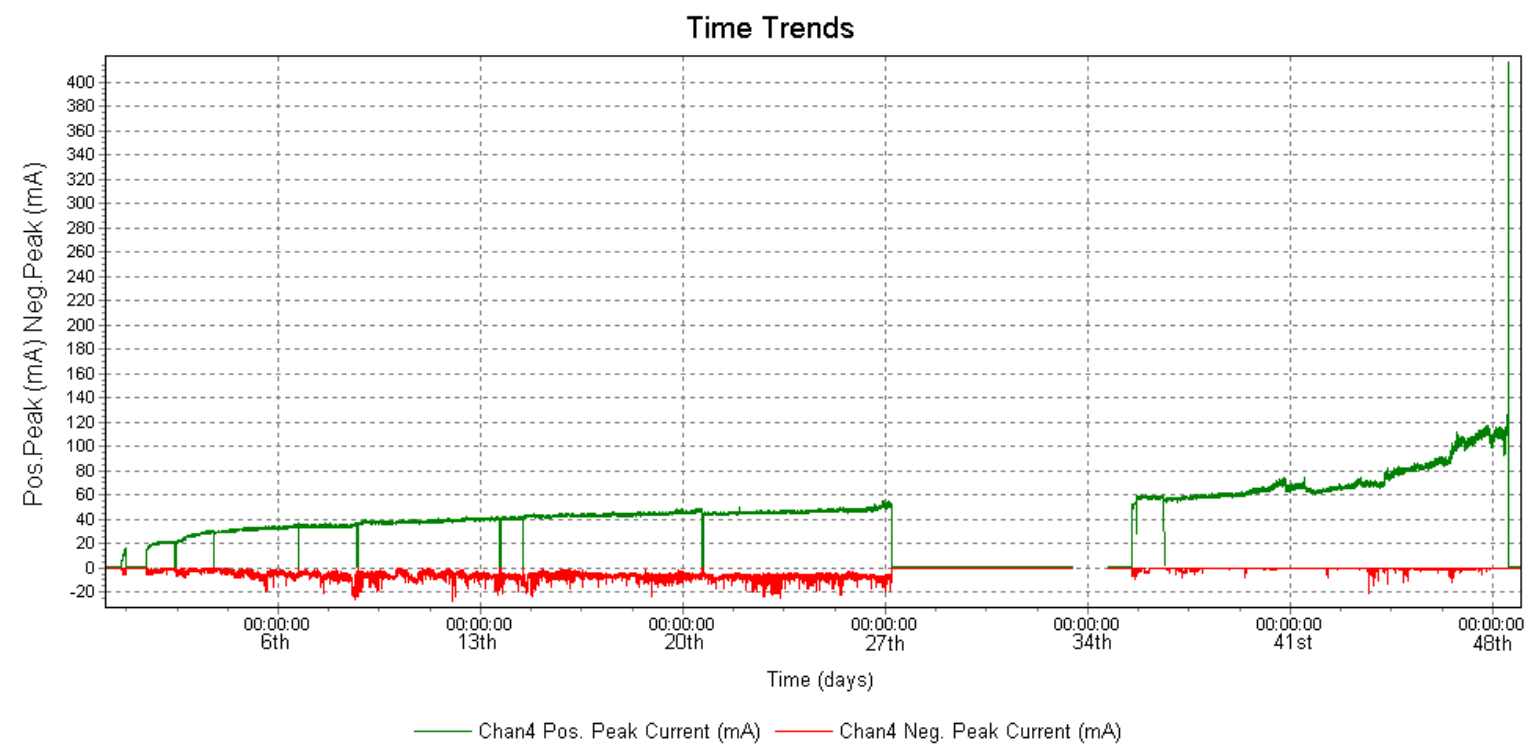

Figure 5.18: Peak current for the HTV SR insulator (channel 4) for positive polarity HVDC excitation

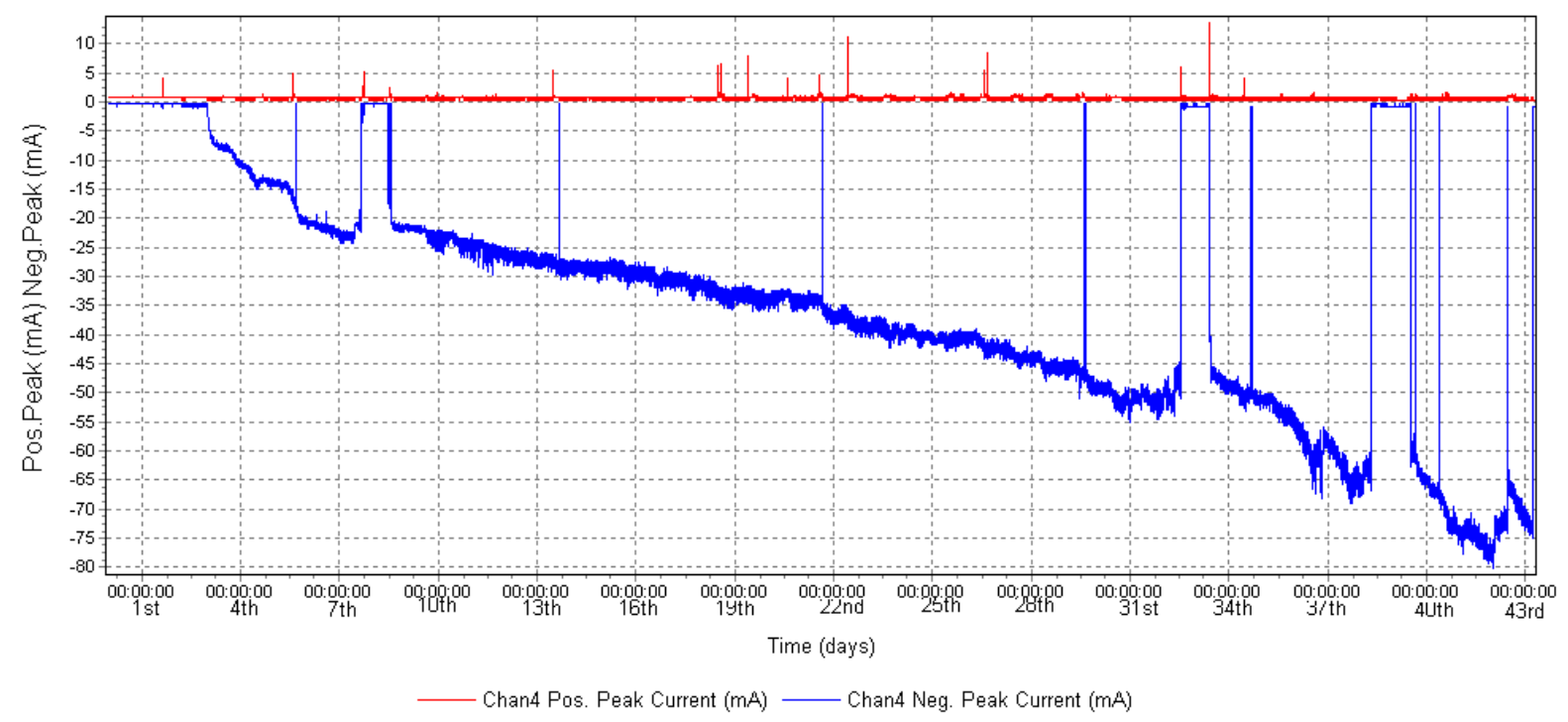

Figure 5.19: Peak current for the HTV SR insulator (channel 4) for negative HVDC excitation

Figure 5.20 compares the hydrophobicity of the HTV SR insulator (channel 4) with a unified SCDof $35 \mathrm{~mm} / \mathrm{kV}$ for HVAC and positive and negative polarity HVDC excitation. The HTV SR insulator (channel 4) fell within a wettability class of 1 for HVAC excitation, a wettability class of 3 for positive polarity HVDC excitation and fell within a wettability class of 5 for negative polarity HVDC excitation. Upon comparison it is shown that the HTV SR insulator (channel 4) retained its hydrophobicity for HVAC excitation, retained part of its hydrophobic properties for 
positive polarity HVDC excitation, but had lost most of its hydrophobic properties for negative polarity HVDC excitation.
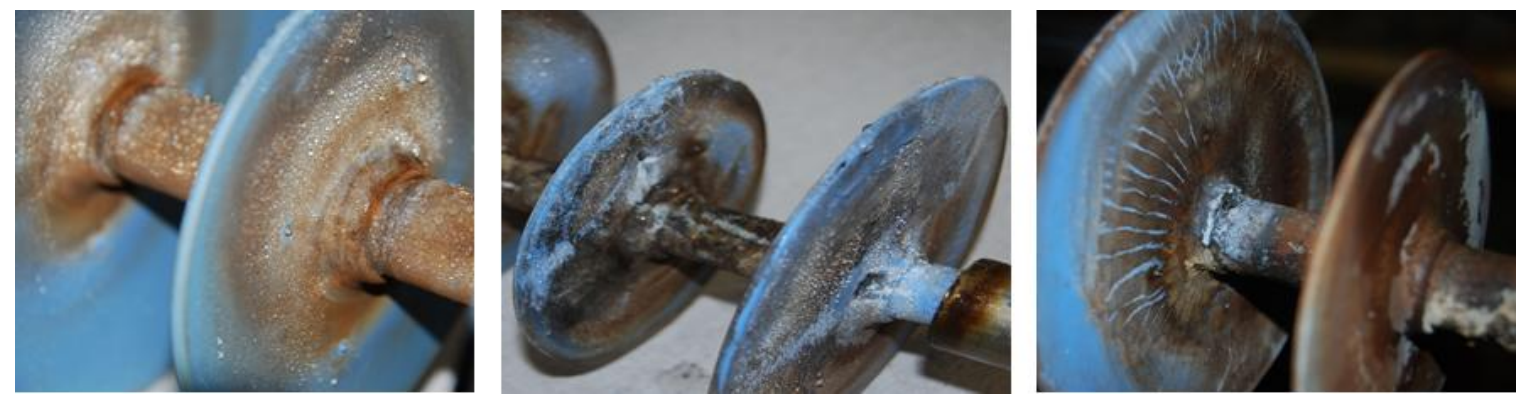

Figure 5.20: Comparison of hydrophobicity for the HTV SR insulator (channel 4) with a unified SCD of $35 \mathrm{~mm} / \mathrm{kV}$ for HVAC and positive and negative polarity HVDC excitation

\subsubsection{Results obtained for the porcelain insulator}

Figure 5.21 compares the material aging of the porcelain insulator (channel 5) with a unified SCDof $28 \mathrm{~mm} / \mathrm{kV}$ for HVAC excitation with that of positive and negative polarity HVDC excitation respectively. The porcelain insulator did not show any significant signs of degradation for HVAC excitation, nor for positive or the negative polarity HVDC excitation. The insulator showed minor discoloration for HVAC excitation, brown discoloration for positive polarity HVDC excitation and reddishbrown discoloration for negative polarity HVDC excitation.
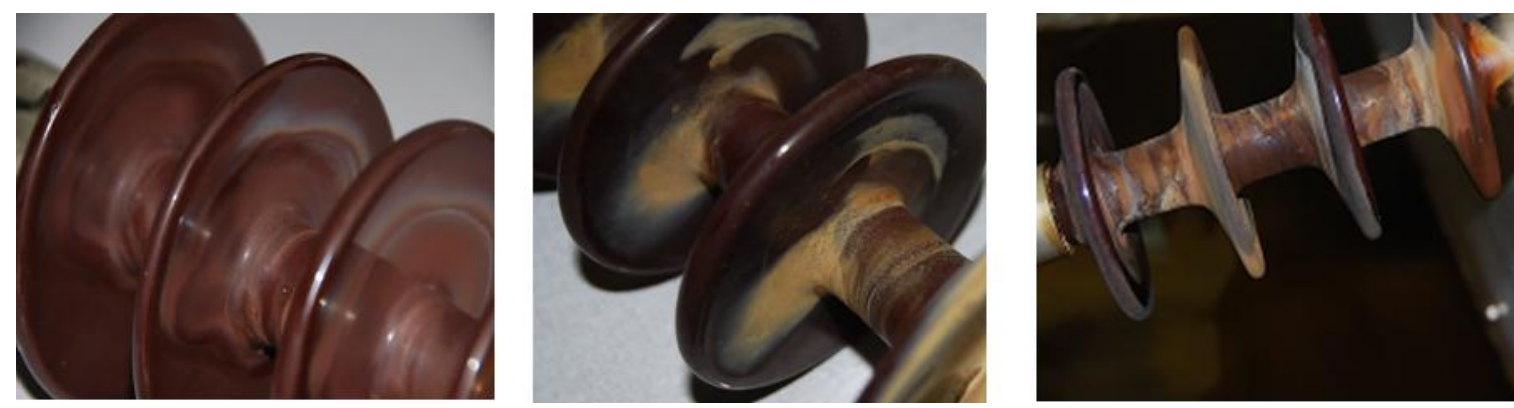

Figure 5.21: Comparison of material aging of the Porcelain insulator (channel 5) for HVAC and positive and negative polarity HVDC excitation

Figure 5.22 shows the initial peak current for the porcelain insulator for HVAC excitation to be $50 \mathrm{~mA}$, with the peak current on the last $\left(53^{\text {rd }}\right)$ day of the test equal to $65 \mathrm{~mA}$ Figure 5.23 shows the initial peak current for the porcelain insulator with positive polarity HVDC excitation to be $37 \mathrm{~mA}$, with the peak current on the last $\left(53^{\text {rd }}\right)$ day of the test equal to $86 \mathrm{~mA}$. Figure 5.24 shows the initial peak current for the porcelain insulator with negative polarity HVDC excitation to be $14 \mathrm{~mA}$, with the peak current on the last $\left(43^{\text {rd }}\right)$ day of the test equal to $60 \mathrm{~mA}$. Initially the porcelain 
insulator had the lowest peak current for negative polarity HVDC excitation, followed by positive polarity HVDC excitation and then HVAC excitation. The leakage current for the porcelain insulator at the end of each test was the highest for positive polarity HVDC excitation, followed by HVAC excitation and then tnegative polarity HVDC excitation.

Table 5.5: Peak currents for the porcelain insulator (channel 5) for HVAC and positive and negative polarity HVDC excitation

\begin{tabular}{|c|c|c|c|}
\hline Insulator type & $\begin{array}{l}\text { Porcelain insulator } \\
\text { (channel 5) with } \\
\text { HVAC excitation }\end{array}$ & $\begin{array}{l}\text { Porcelain insulator } \\
\text { (channel 5) with } \\
\text { positive HVDC } \\
\text { excitation }\end{array}$ & $\begin{array}{l}\text { Porcelain insulator } \\
\text { (channel 5) with } \\
\text { negative HVDC } \\
\text { excitation }\end{array}$ \\
\hline $\begin{array}{l}\text { Peak current }[\mathrm{mA}] \text { in } \\
\text { the beginning }\end{array}$ & $50 \mathrm{~mA}$ & $37 \mathrm{~mA}$ & $14 \mathrm{~mA}$ \\
\hline $\begin{array}{l}\text { Peak current }[\mathrm{mA}] \text { in } \\
\text { the end }\end{array}$ & $65 \mathrm{~mA}$ & $86 \mathrm{~mA}$ & $60 \mathrm{~mA}$ \\
\hline Hydrophobicity lost & $\begin{array}{l}\text { Immediately after test } \\
\text { voltage is applied. }\end{array}$ & $2 \mathrm{~h} 30$ minutes later & 2 days later \\
\hline $\begin{array}{lrr}\begin{array}{l}\text { Duration of } \\
\text { experiment } \\
\text { insulator trip }\end{array} & \begin{array}{r}\text { the } \\
\text { before }\end{array} \\
\end{array}$ & 52 days & 41 days & 41 days \\
\hline
\end{tabular}

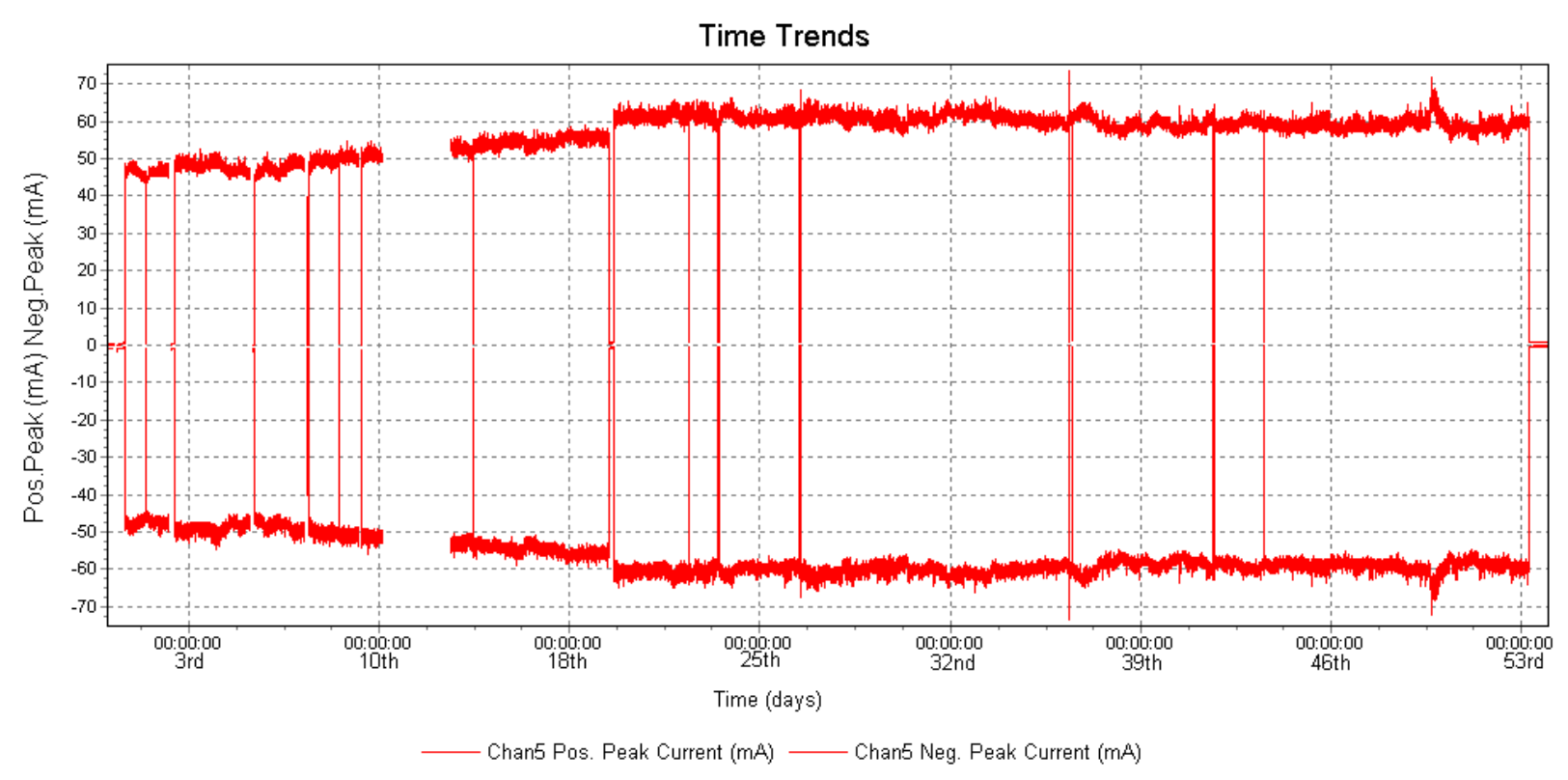

Figure 5.22: Peak current for the porcelain insulator (channel 5) for HVAC excitation 


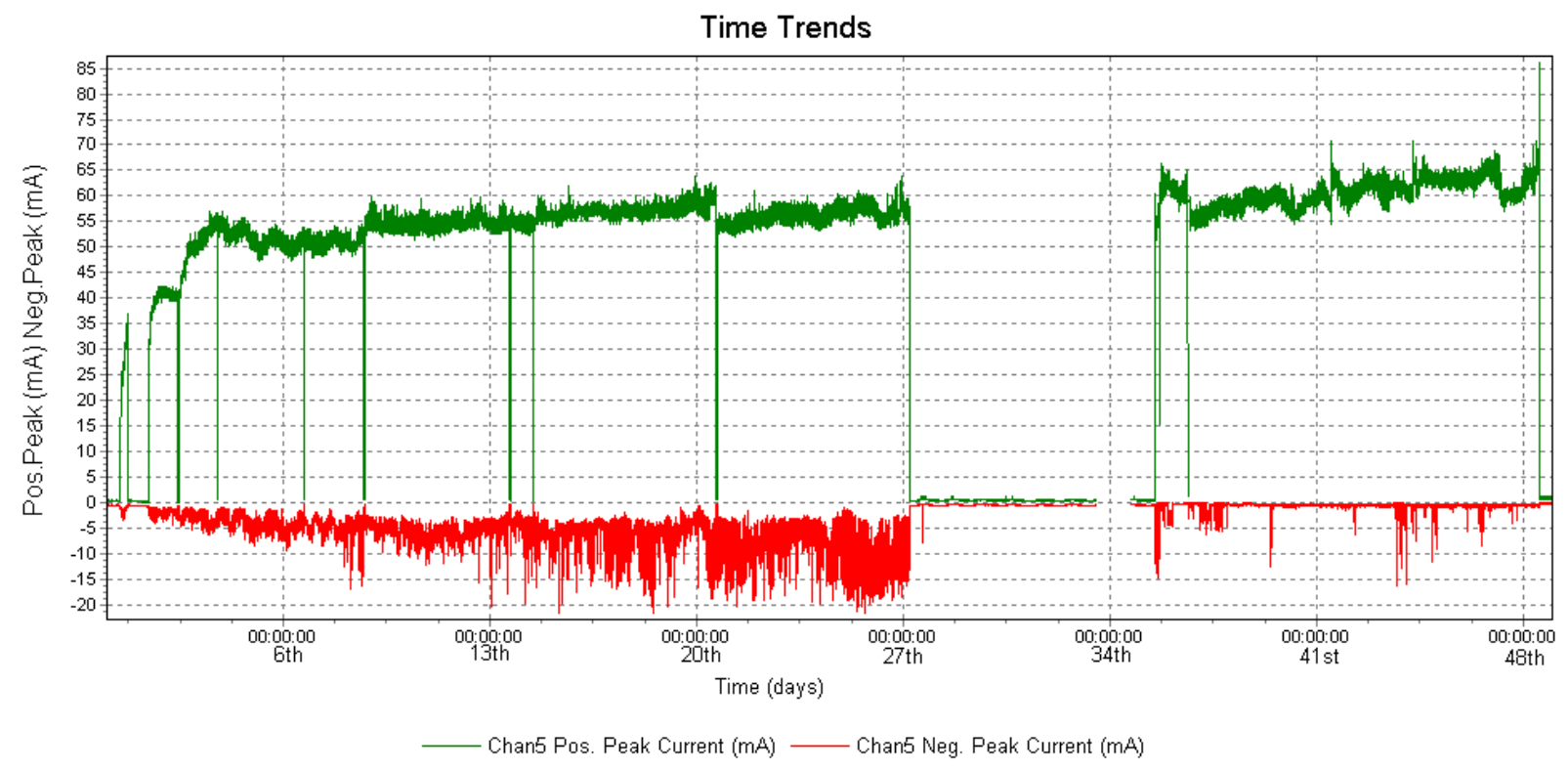

Figure 5.23: Peak current for the porcelain insulator (channel 5) for positive polarity HVDC excitation

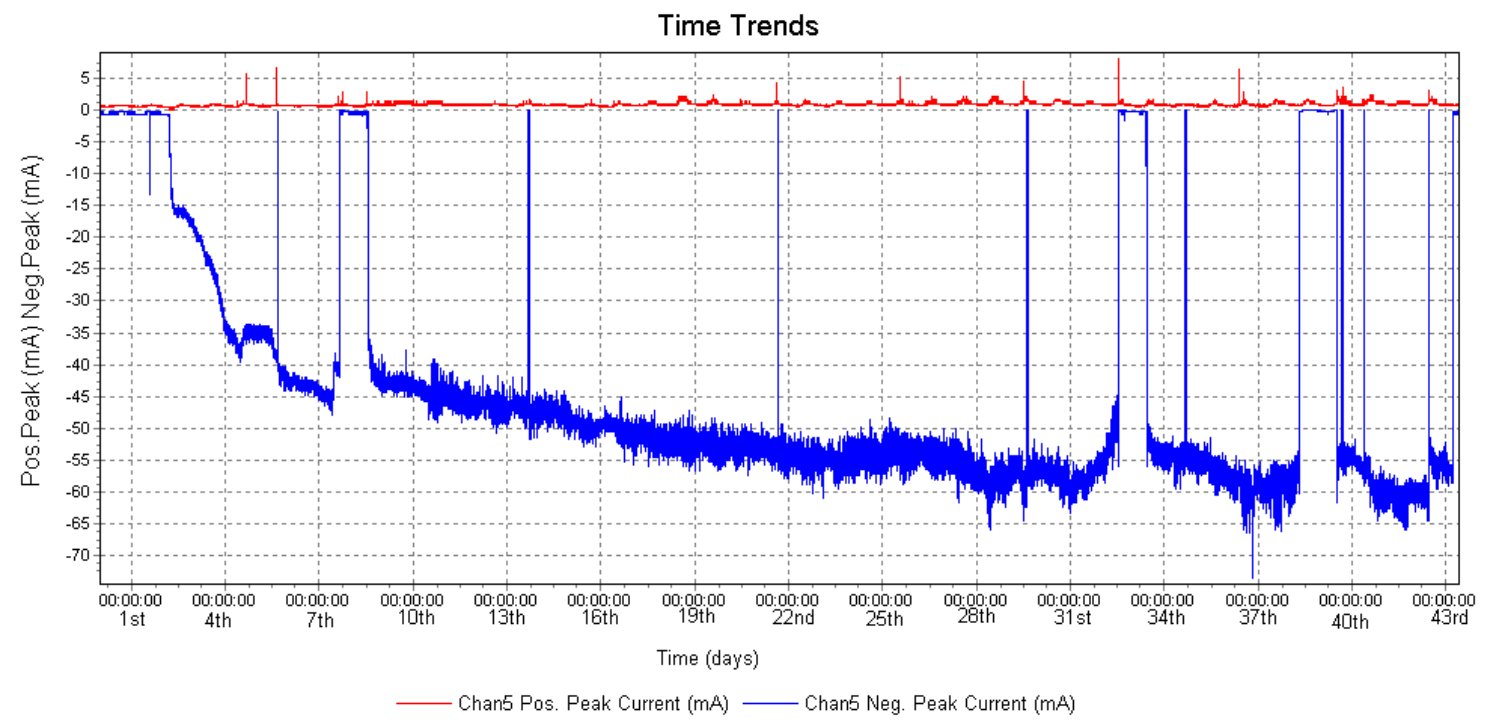

Figure 5.24: Peak current for the porcelain insulator (channel 5) for negative polarity HVDC excitation

Figure 5.25 compares the hydrophobicity of the porcelain insulator (channel 5) with a unified SCDof $28 \mathrm{~mm} / \mathrm{kV}$ for HVAC and positive and negative polarity HVDC excitation. The porcelain insulator (Channel 5) fell within a wettability class of 6 for the HVAC and positive and negative polarity HVDC excitation. The insulator remained completely hydrophilic, irrespective of the type of excitation. 

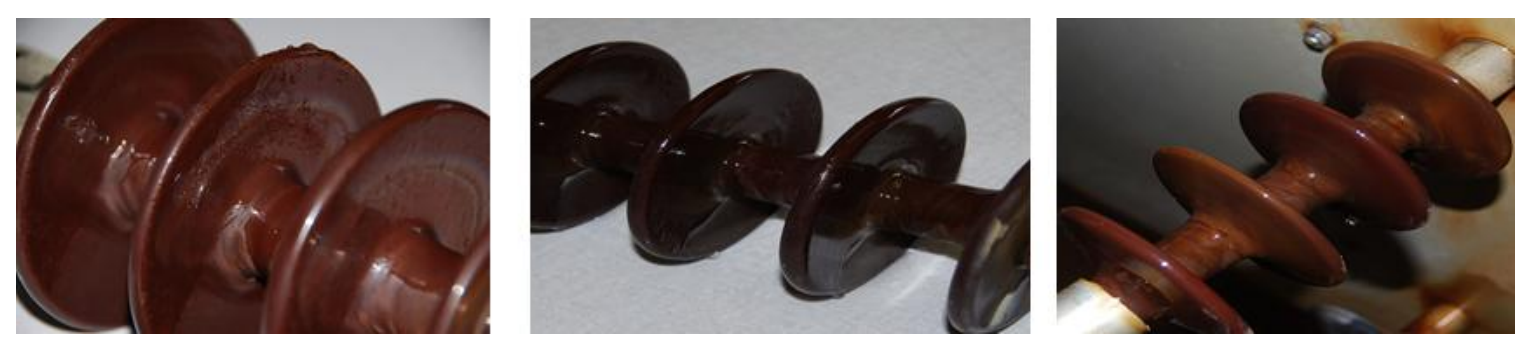

Figure 5.25: Comparison of hydrophobicity for the porcelain insulator (channel 5) with a unified SCD of $28 \mathrm{~mm} / \mathrm{kV}$ for HVAC and positive and negative polarity HVDC excitation

\subsubsection{Results obtained for the RTV SR coated porcelain insulator}

Figure 5.26 compares the material aging of the RTV SR coated porcelain insulator (channel 6) with a unified SCDof $28 \mathrm{~mm} / \mathrm{kV}$ for HVAC excitation with that for positive and negative polarity HVDC excitation respectively. The RTV SR coated porcelain insulator had severe tracks, erosion and discoloration on the insulator for HVAC excitation. The coating material had been completely removed from the rods, with some parts of the coating on the sheds remaining intact. The RTV SR coated porcelain insulator (Channel 6) showed severe tracks, discoloration, as well as erosion to the coating material for positive polarity HVDC excitation. The coating had been peeled from the surface. The RTV SR coated porcelain insulator (Channel 6) had less tracks and erosion, as well as slight discoloration of the surface for negative polarity HVDC excitation. The peeling of the coating was not as severe as for positive polarity HVDC and the HVAC excitation. The RTV SR coated porcelain aged the worst for HVAC excitation, followed by positive polarity HVDC excitation and then negative polarity HVDC excitation.
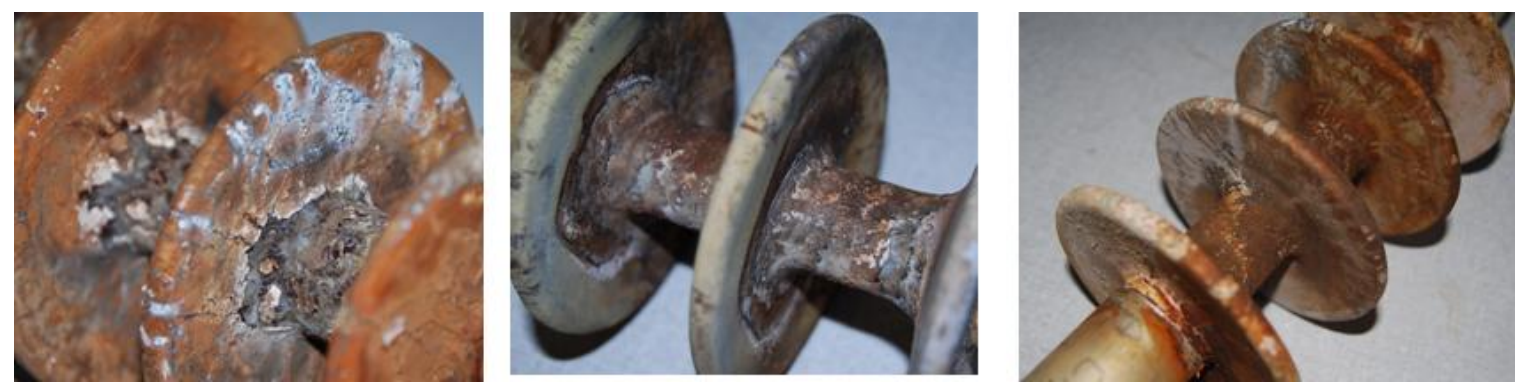

Figure 5.26: Comparison of material aging of the RTV SR coated porcelain insulator (channel 6) for HVAC and positive and negative polarity HVDC excitation

Figure 5.27 shows the initial peak current for the RTV SR coated porcelain insulator for HVAC excitation to be $60 \mathrm{~mA}$, with the peak current on the last $\left(53^{\text {rd }}\right)$ day of the test equal to $220 \mathrm{~mA}$. Figure 5.28 shows the initial peak current for the RTV SR coated porcelain insulator for positive polarity HVDC excitation to be $50 \mathrm{~mA}$, with 
the peak current on the last $\left(28^{\text {th }}\right)$ day of the test equal to $725 \mathrm{~mA}$. Figure 5.29 shows the initial peak current for the RTV SR coated porcelain insulator for negative polarity HVDC excitation to be $40 \mathrm{~mA}$, with the peak current on the last $\left(32^{\text {nd }}\right)$ day of the test equal to $700 \mathrm{~mA}$. Initially, the negative polarity HVDC excitation showed the lowest leakage current value (current only started to show on the $6^{\text {th }}$ day), followed by the positive polarity HVDC excitation and then the HVAC excitation. The leakage current for the RTV SR coated porcelain insulator at the end of the HVAC test rose steadily to a value of $220 \mathrm{~mA}$ after 53 days. For positive polarity HVDC excitation a value of $724 \mathrm{~mA}$ was reached when the insulator failed after 28 days. For negative polarity HVDC excitation a value of $700 \mathrm{~mA}$ was reached when the insulator failed after 31 days.

Table 5.6: Peak currents for the RTV SR coated porcelain insulator (channel 6) for HVAC and positive and negative polarity HVDC excitation

\begin{tabular}{|l|l|l|l|}
\hline Insulator type & $\begin{array}{l}\text { RTV SR coated } \\
\text { porcelain insulator } \\
\text { (channel 6) with } \\
\text { HVAC excitation }\end{array}$ & $\begin{array}{l}\text { RTV SR coated } \\
\text { porcelain insulator } \\
\text { (channel 6) with } \\
\text { positive HVDC } \\
\text { excitation }\end{array}$ & $\begin{array}{l}\text { RTV SR coated } \\
\text { porcelain insulator } \\
\text { channel 6) with } \\
\text { negative HVDC } \\
\text { excitation }\end{array}$ \\
\hline $\begin{array}{l}\text { Peak current [mA] in } \\
\text { the beginning }\end{array}$ & $60 \mathrm{~mA}$ & $40 \mathrm{~mA}$ \\
\hline $\begin{array}{l}\text { Peak current [mA] in } \\
\text { the end }\end{array}$ & $220 \mathrm{~mA}$ & $\begin{array}{l}725 \mathrm{~mA} \\
\text { "tripped" }\end{array}$ & "tripped" \\
\hline $\begin{array}{l}\text { Hydrophobicity lost } \\
\text { the days later }\end{array}$ & Immediately after test \\
voltage is applied. & days later & 31 days \\
\hline $\begin{array}{l}\text { Duration oxperiment before } \\
\text { insulator trip }\end{array}$ & 52 days & 28 days & \\
\hline
\end{tabular}

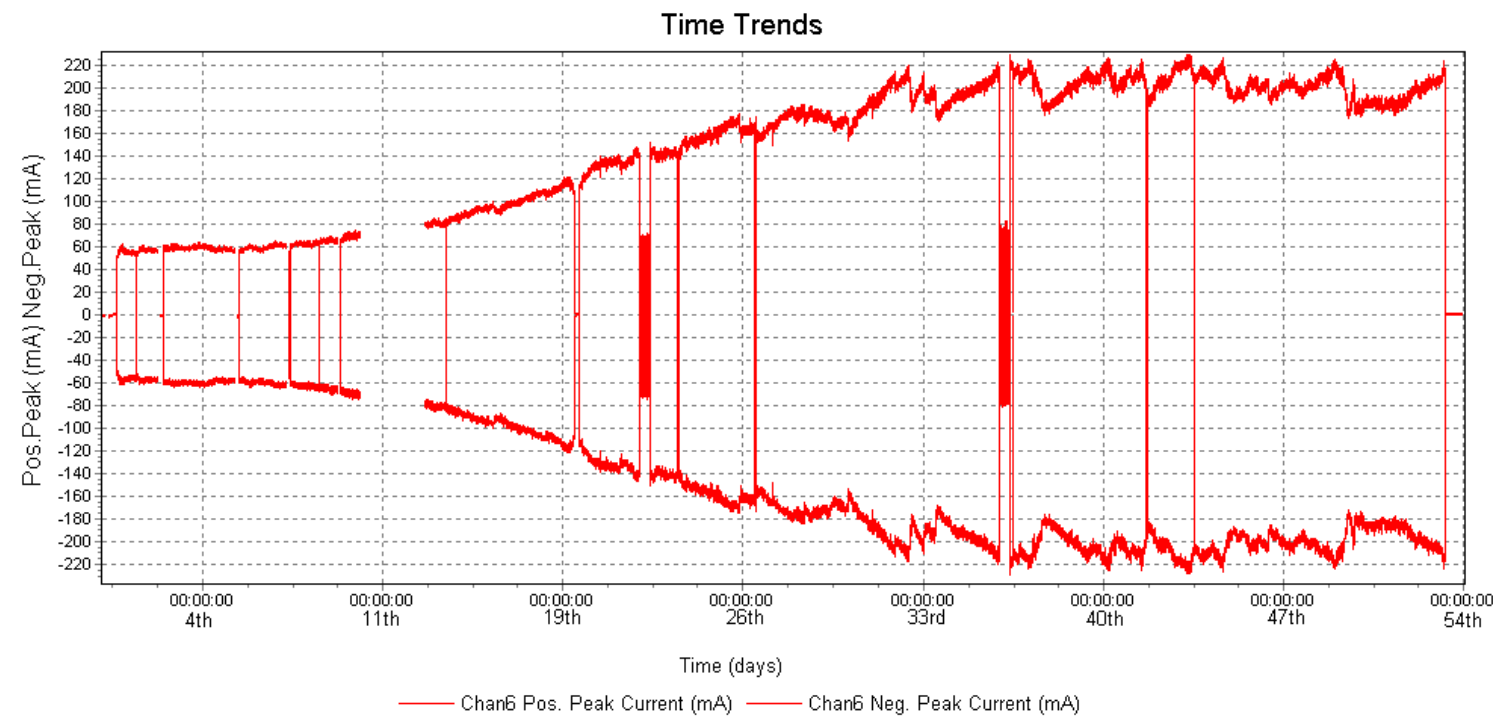


Figure 5.27: Peak current for the RTV SR coated porcelain insulator (channel 6) for HVAC excitation

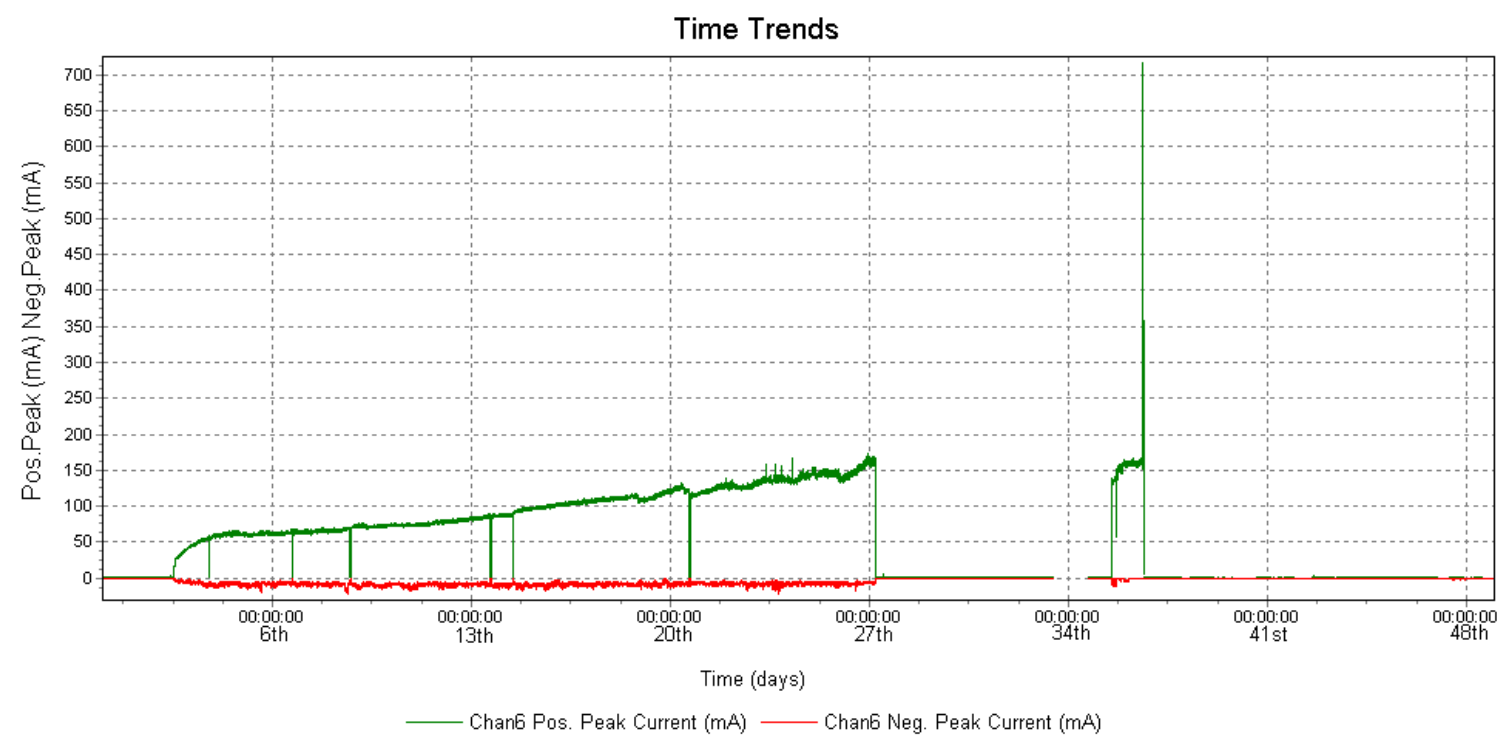

Figure 5.28: Peak current for the RTV SR coated porcelain insulator (channel 6) for positive polarity HVDC excitation

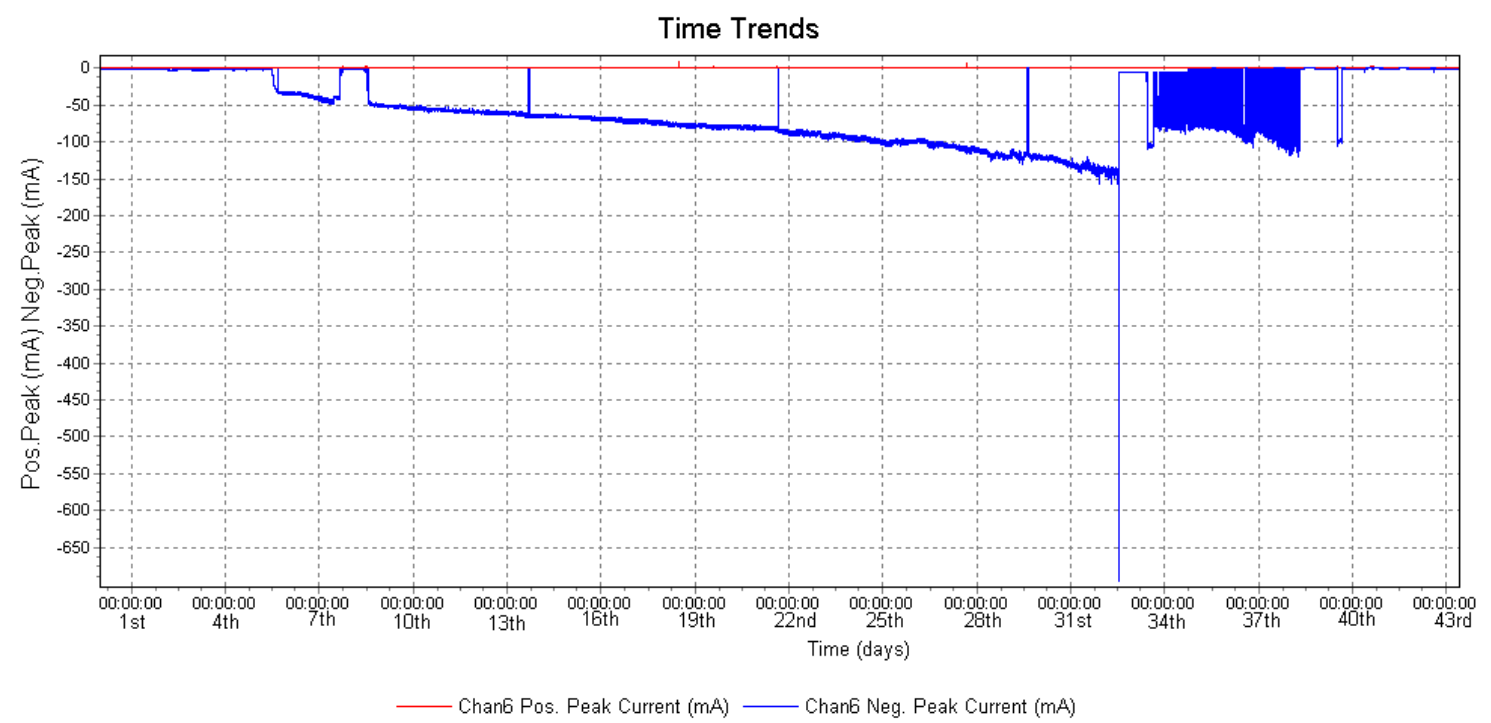

Figure 5.29: Peak current for the RTV SR coated porcelain insulator (channel 6) for negative polarity HVDC excitation

Figure 5.30 compares the hydrophobicity of the RTV SR coated porcelain insulator (channel 6) with a unified SCDof $28 \mathrm{~mm} / \mathrm{kV}$ for HVAC and positive and negative polarity HVDC excitation. The RTV SR coated porcelain insulator (channel 6) fell within a wettability class of 3 for HVAC excitation, a wettability class of 4 for positive HVDC excitation and a wettability class of 4 for negative HVDC excitation. 

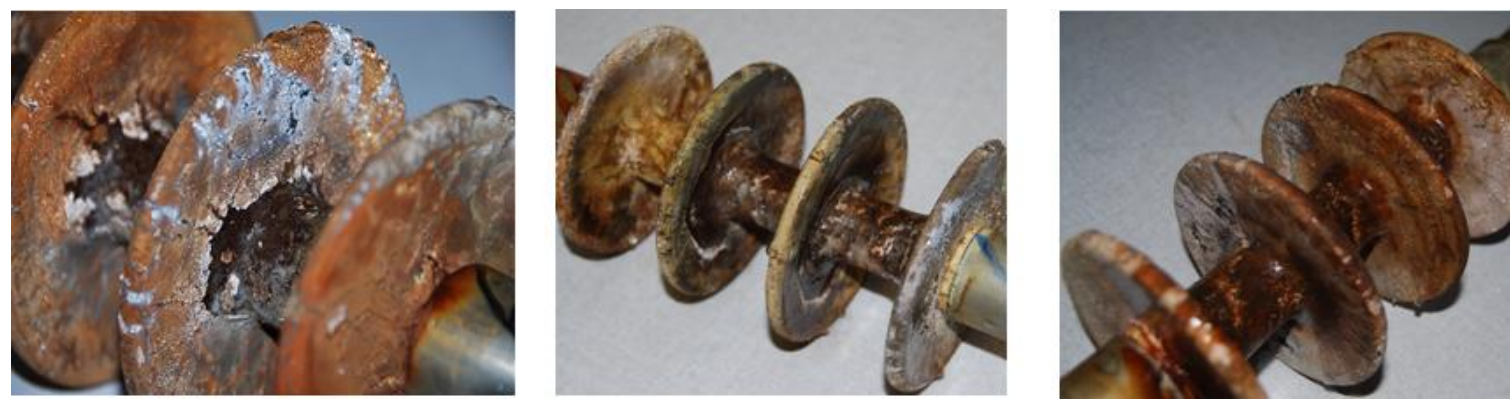

Figure 5.30: Comparison of hydrophobicity for the RTV SR coated porcelain insulator (channel 6) with a unified SCD of $28 \mathrm{~mm} / \mathrm{kV}$ for HVAC and positive and negative polarity HVDC excitation 


\section{Conclusions and Recommendations}

\subsection{Introduction}

The conclusions and recommendations based on each section will be discussed in detail in this chapter. These conclusions and recommendations are based on the results obtained with HVAC excitation for test series 1 and with HVAC and positive and negative polarity HVDC excitation for test series 2 .

\subsection{Conclusions and recommendations for test series 1 with HVAC excitation}

The results obtained from the first experiment with the TWT with HVAC excitation give rise to the following conclusions:

- As expected, the insulators with the shortest creepage distance have the highest maximum peak current for RTV SR coated glass and glass samples.

- $\quad$ For the glass samples, there is a visible reduction in peak currents whenever the salt-water solution level decreases.

- For the RTV SR coated glass samples, the peak currents remain fairly constant, even when the salt-water level drops.

- $\quad$ For a creepage distance of $277 \mathrm{~mm}$, the glass insulator (channel 1) has a higher peak current when compared to the RTV SR coated glass insulator (channel 2).

- $\quad$ For a creepage distance of $346 \mathrm{~mm}$, the RTV SR coated glass sample (channel 4) has lower initial peak currents compared to the glass insulator (channel 3). The maximum peak currents for the RTV SR coated glass increase as the test progresses. Towards the end, however, there is a small decrease in the maximum peak currents when compared to the glass insulator.

- $\quad$ For a creepage distance of $433 \mathrm{~mm}$, the RTV SR coated glass sample (channel 5) has higher peak currents compared to glass insulator (channel 6) throughout the test duration.

- It is recommended that a temperature controller be designed to keep the temperature constant at $25 \pm 5^{\circ} \mathrm{C}$.

- A water system, designed to regulate the level of the salt-water solution, is required. 


\subsection{Conclusions and recommendations for test series 2 with HVAC excitation}

The following conclusions were made based on the peak leakage currents for the modified HVAC test:

- $\quad$ The EPDM insulator (channel 1) had higher peak currents than the HTV SR insulator (channel 2) in the beginning. As the hydrophobicity was lost, however, the HTV SR insulator began to have higher peak currents than the EPDM insulator (Channel 1).

- $\quad$ The EPDM insulator (channel 1) showed better overall performance compared to the HTV SR insulator (channel 2) in terms of leakage currents.

- $\quad$ The HTV SR insulator with a USCDof $28 \mathrm{~mm} / \mathrm{kV}$ (channel 3) performed much worse than the HTV SR insulator with a USCDof $35 \mathrm{~mm} / \mathrm{kV}$ in terms of leakage currents. This showed that the insulators with a shorter creepage distance have higher peak currents than the insulators with longer creepage distances.

- $\quad$ The occurrence of each new puncture hole through the HTV SR insulator sheds (Channel 3) brought about increasingly higher peak currents, causing a fast rise in the peak current and eventual failure of the sample.

- The presence of a coating seemed to increase the leakage current as soon as loss of hydrophobicity occurred. It appears that the RTV SR coated porcelain (Channel 6) performed much worse than the porcelain insulator (Channel 5) in terms of peak leakage currents.

There following conclusions were made based on material aging:

- $\quad$ The EPDM insulator (channel 1) and the HTV SR insulator (channel 2) aged in differently. Both the HTV SR insulator and the EPDM insulator showed signs of brown discoloration. The EPDM insulator (Channel 1) showed erosion and small brown bubbles across the mould lines. The HTV SR insulator (Channel 2) had darkening as well as localised erosion on the mould lines.

- $\quad$ The HTV SR insulator with a USCDof $28 \mathrm{~mm} / \mathrm{kV}$ (channel 3) experienced five punctures, discoloration, and had tracking like marks on its surface. The HTV SR insulator with a USCDof $35 \mathrm{~mm} / \mathrm{kV}$ (channel 4) showed discolouration as well as tracking like marks on its surface. Both these insulators showed less 
severe discolouration and surface markings than that of the moulded EPDM and HTV SR insulators with the same profile (channels 1 and 2)

- The RTV SR coated porcelain (channel 6) had reddish brown surface discolouration with the coating severely degraded and partly stripped from the surface of the insulator. The reference porcelain insulator (channel 5) showed only slight surface discoloration.

The following conclusions were made based on the hydrophobicity assessment:

- The HTV SR insulator (Channel 2) retained its hydrophobicity as it had recovered to a wettability class of 1 . The EPDM insulator (channel 1) remained hydrophilic, falling within a wettability class of 5 .

- The HTV SR insulator with a USCDof $35 \mathrm{~mm} / \mathrm{kV}$ (channel 4) retained its hydrophobicity much better when compared to the HTV SR insulator with USCD of $28 \mathrm{~mm} / \mathrm{kV}$ (channel 3). The HTV SR insulator with the shorter creepage distance (channel 3) had recovered to a wettability class of either 3 or 4, whereas the insulator with the longer creeepage distance recovered to a wettability class of 1 .

- The porcelain insulator remained completely hydrophilic (wettability class of 6), whereas the RTV SR coated porcelain insulator (channel 6) had recovered to a wettability class of 4 . It is observed that coating improves the hydrophobicity of the insulator. This type of coating cannot endure the continued high stresses created by the tracking wheel tester.

\subsection{Conclusions and recommendations for test series 2 with positive polarity HVDC excitation}

The following conclusions were made based on the peak leakage currents for the positive polarity HVDC test:

- $\quad$ The HTV SR insulator (Channel 2) performed much better than the EPDM (Channel 1) insulator up to the point when its hydrophobicity had been lost.

- The EPDM insulator (Channel 1) performed much better than the HTV SR insulator (Channel 2), once the hydrophobicity of the HTV SR insulator was lost. This showed that insulators made of different materials perform 
differently in terms of leakage current. The state of hydrophobicity governs the leakage current performance of the insulators.

- The HTV SR insulator with a USCDof $28 \mathrm{~mm} / \mathrm{kV}$ from manufacturer A (channel 2) was compared with the HTV SR insulator with a USCDof 28 $\mathrm{mm} / \mathrm{kV}$ from manufacturer B (channel 3) to determine the effect that different profiles have on the performance of the insulators. It was shown that the HTV SR insulator of manufacturer A performed better than that of manufacturer B, showing that profile plays an important role in the performance of the insulators under HVDC stresses of positive polarity.

- The effect of USCD on the performance of insulators for positive polarity HVDC stress was investigated. The HTV SR insulator (channel 3) with a USCDof $28 \mathrm{~mm} / \mathrm{kV}$ performed much better than the (HTV SR insulator (channel 4) with a USCDof $35 \mathrm{~mm} / \mathrm{kV}$. By evaluation of the data it was concluded that the insulator with the shortest USCD (Channel 3) performed worse than the insulator with the longer USCD (Channel 4).

- The effect of a RTV SR coating on the performance of insulators for positive polarity HVDC stress was evaluated. It was found that coating the insulator with a RTV SR coating improved the initial performance of the insulator with regards to leakage current, however after the hydrophobicity of the coating had been lost, the RTV SR coated porcelain insulator (channel 6) performed worse than the uncoated porcelain insulator.

There following conclusions were made based on material aging for the positive polarity HVDC test:

- When comparing the EPDM insulator (channel 1) with the HTV SR insulator (channel 2), it is shown that the two insulators, being of different materials having the same profile, have different aging modes. The EPDM insulator (channel 1) showed signs of heavy erosion and discoloration, whereas the HTV SR insulator (channel 2) showed heavier erosion in a tracking like pattern penetrating deep into the material. The HTV SR insulator (channel 2) experienced a puncture toward the end of the test cycle. Both insulators showed signs of discoloration. It appears as if the HTV SR insulator (channel 2) had aged much worse when compared to the EPDM insulator (channel 2). 
- The effect of different profiles and manufacturers, hence material formulations, on the performance of insulators under positive HVDC stress was evaluated. It may be shown that the HTV SR insulator (channel 2) from manufacturer A performed much better than the HTV SR insulator (channel 3) from manufacturer $B$ in terms of material aging. The HTV SR insulator of manufacturer A (channel 2) had a puncture, severe erosion and tracking like discolouration on its surface, whereas the HTV SR insulator of manufacturer B (channel 3) had lots of punctures with part of the rod exposed due to erosion caused by the insulator flashing over.

- The effect of different USCD on the performance of insulators under positive polarity HVDC stress was evaluated. The HTV SR insulator (channel 3) with a USCDof $28 \mathrm{~mm} / \mathrm{kV}$ performed worse than the HTV SR insulator (channel 4) with a USCDof $35 \mathrm{~mm} / \mathrm{kV}$. The HTV SR insulator with a USCD of $28 \mathrm{~mm} / \mathrm{kV}$ showed continual degradation in the form of puncture marks until the insulator failed exposing the rod section core. The HTV SR insulator with a USCD of 35 $\mathrm{mm} / \mathrm{kV}$ had approximately eight puncture marks.

- $\quad$ The effect of a RTV SR coating on the performance of insulators for positive polarity HVDC stress was evaluated. It was found that the RTV SR coated porcelain insulator (channel 6) seemed to age faster than the uncoated porcelain insulator (Channel 5), which did not show any signs of degradation. Both the coated and the uncoated insulators showed signs of discolouration, whereas the RTV SR coated porcelain insulator (channel 6) showed parts of the coating to have been cleared off or removed from the insulator surface.

The following conclusions were made based on the hydrophobicity assessment for the positive polarity HVDC test:

- The effect of different materials on the hydrophobicity performance was evaluated. The EPDM insulator had completely lost its hydrophobicity, whereas the HTV SR insulator retained some of its hydrophobic properties.

- When evaluating the effect of different profiles on the hydrophobicity performance of insulators it was shown that the HTV SR insulator (channel 2) from manufacturer A retained its hydrophobicity much better than the HTV SR insulator from manufacturer B (channel 3). 
- The effect of different USCD on the hydrophobicity performance of insulators under positive polarity HVDC stress was evaluated. It is shown that the HTV SR insulator with an USCDof $35[\mathrm{~mm} / \mathrm{kV}]$ (channel 4), retained its hydrophobicity much better than HTV SR insulator with an USCD of 28 $[\mathrm{mm} / \mathrm{kV}]$ (channel 3).

- The effect of a RTV SR coating on the hydrophobicity performance of insulators was evaluated. The RTV SR coated porcelain insulator (channel 6) had retained some of its hydrophobic properties, whereas the uncoated porcelain insulator (channel 5), being of a hydrophilic nature, showed no change.

\subsection{Conclusions and recommendations for test series 2 with negative polarity HVDC excitation}

The following conclusions were made based on the peak leakage currents for the negative polarity HVDC test:

- The HTV SR insulator (channel 2) had lower initial peak currents when compared to the EPDM insulator (Channel 1). After the hydrophobicity of the HTV SR insulator was lost, however, the EPDM insulator (channel 1) had lower peak currents compared to the HTV SR insulator.

- $\quad$ The effects of different manufacturers, hence material formulations, and profiles on the leakage current performance of insulators were evaluated. It was shown that the HTV SR insulator of manufacturer A (channel 2) had higher currents than the HTV SR insulator of manufacturer B (channel 3) in the beginning, but at the end the HTV SR insulator of manufacturer B (channel 3) showed much higher leakage currents when compared to the insulator of manufacturer B, having a different profile and material formulation.

- The effect of different USCDon the performance of insulators of the same material type and manufacturer, using leakage current data was evaluated. The insulator with the shortest USCD (Channel 3) had higher peak currents when compared to the insulator with the longer USCD (Channel 4) throughout the duration of the experiment. The insulator with the longer USCD (channel 4) took longer to develop a measureable leakage current when compared to the insulator with the shorter USCD (channel 3). 
- The effect of a RTV SR coating on the leakage current performance of insulators was evaluated. It is concluded that once the hydrophobic property of the RTV SR coated porcelain insulator (channel 6) had been lost, the peak currents had risen to a higher level when compared to the porcelain insulator (channel 5).

There following conclusions were made based on material aging for the negative polarity HVDC test:

- The effect of materials and the different aging modes of insulators was evaluated. The HTV SR insulator (channel 2) performed worse when compared to the EPDM insulator (channel 1). The HTV SR insulator had severe erosion and tracking like discoloration on its surface layer with narrow yet deep erosion on its mould lines. The EPDM insulator (channel 1) showed signs of discoloration and severe erosion, especially on the mould lines.

- The effect of different profiles and manufacturers, hence material formulations, on the aging performance of insulators was evaluated. The HTV SR insulator from manufacturer A (channel 2) aged relatively slow when compared to the HTV SR insulator from manufacturer B (channel 3). The insulator from manufacturer A (channel 2) had one puncture mark, whereas the insulator from manufacturer B (channel 3) had about 4 puncture marks. Both insulators had tracking like patterns in the form of a star on their surface layers, with slight erosion visible along these patterns.

- Insulators of the same manufacturer and material type having different USCD were evaluated with regards to their aging performance. The HTV SR insulator with a USCD of $28 \mathrm{~mm} / \mathrm{kV}$ (channel 3) seemed to have aged worse when compared to the HTV SR insulator with a USCD of $35 \mathrm{~mm} / \mathrm{kV}$. Both the insulators had puncture marks and tracking like discoloration in the form of a star on the surface layer. The insulator with the shorter USCD (channel 3) had been severely damaged by the fault current when it failed exposing the rod core of the insulator.

- The effect of a RTV SR coating on the aging performance of insulators was evaluated. The uncoated porcelain insulator (channel 5) showed no signs of degradation other than light brown discoloration on the surface. The RTV SR 
coated porcelain insulator (channel 6) showed signs of severe erosion with tracking like patterns and a light brown discoloration on the surface. The RTV SR coating showed severe aging, whereas the uncoated insulator showed no degradation.

The following conclusions were made based on the hydrophobicity assessment for the negative polarity HVDC test:

- The effect of different materials on the hydrophobicity of insulators having the same profile was evaluated. Both the EPDM insulator (channel 1) and the HTV SR insulator (channel 2) reflect a wettability class of 5, implying that both insulators are almost completely hydrophilic.

- The effect of different profiles and manufacturers, hence material formulations, on the hydrophobicity performance of insulators was evaluated. The HTV SR insulator of manufacturer A (channel 2) had a wettability class of 5, whereas the HTV SR insulator from manufacturer B (channel 3) had a wettability class of 4. This means that the insulator of manufacturer B (channel 3) had retained its hydrophobic properties better than the HTV SR insulator from manufacturer A (channel 2). This shows that different profiles and manufacturers have an effect on the hydrophobicity performance of insulators.

- $\quad$ Insulators of the same material type and manufacturer, having different $\mathrm{US}_{C D}$, were evaluated with regard to their hydrophobicity performance. The HTV SR insulator with a USCDof $28 \mathrm{~mm} / \mathrm{kV}$ (channel 3) had a wettability class of 4 , whereas the HTV SR insulator with a USCDof $35 \mathrm{~mm} / \mathrm{kV}$ (channel 4) had a wettability class of 5 . This showed that the insulator with the shorter USCDrecovers its hydrophobicity much better than the insulator with the longer USCD.

- The effect of an RTV SR coating on the hydrophobicity performance of porcelain insulators was evaluated. The uncoated insulator (channel 5) had a wettability class of 6 (completely hydrophilic), whereas the coated insulator (channel 6) had a wettability class of 4 (aproaching a hydrophilic state). It is concluded that a RTV SR coating improves the hydrophobicity of the insulator. 


\subsection{Conclusions and recommendations based on comparison between the results for different excitation voltages}

This section gives conclusions and recommendations based upon a comparison of the tests conducted for different excitation voltages:

- $\quad$ The aging performance of the EPDM insulator (Channel 1) for HVAC and positive and negative polarity HVDC excitation was evaluated. It can be concluded that the EPDM insulator (channel 1) aged the worst for the positive polarity HVDC stress, followed by the negative polarity HVDC stress and finally the HVAC excitation.

- In terms of peak current, the EPDM insulator had the highest peak current for negative polarity HVDC stress in the beginning, followed by positive polarity HVDC stress and then HVAC stress. Toward the end of the experiments, the positive polarity HVDC stress had the highest peak currents, followed by the negative polarity HVDC stress and HVAC stress.

- The EPDM insulators had attained a wettability class of 5 for all the excitation types, i.e. HVAC, positive polarity HVDC and negative polarity HVDC.

- The HTV SR insulator showed higher initial leakage currents for HVAC stress, followed by the positive polarity HVDC stress and then negative polarity HVDC stress. Toward the end of the experiments, the HTV SR insulator with positive polarity HVDC stress test had the highest peak current, followed by HTV SR insulator for the negative polarity HVDC stress and then the HVAC stress.

- The HTV SR insulator (channel 2) had a wettability class of 1 for HVAC excitation, with a wettability class of 3 for positive polarity HVDC excitation and a wettability class of 5 for negative polarity HVDC excitation. This meant that the HTV SR insulator retained its hydrophobic properties better for HVAC excitation, followed by positive polarity HVDC excitation and then negative polarity HVDC excitation.

- In terms of material aging, the HTV SR insulator from manufacturer B (channel 3) performed worst for positive polarity HVDC excitation, followed by negative polarity HVDC excitation, and the best for the HVAC excitation. 
- The HTV SR insulator with a USCD of $28 \mathrm{~mm} / \mathrm{kV}$ (Channel 3) had the lowest peak currents for HVAC excitation, followed by negative polarity HVDC excitation and eventually positive polarity HVDC excitation. Furthermore, the HTV SR insulator took a longer time to trip for HVAC excitation than for negative polarity HVDC excitation. It took a very short time before the insulator tripped for positive polarity HVDC excitation.

- The HTV SR insulator had a wettability class of 2 for HVAC excitation, a wettability class of 3 for positive polarity HVDC excitation and a wettability class of 4 for negative polarity HVDC excitation. The HTV SR insulator seems to retain its hydrophobicity better for HVAC excitation, followed by positive polarity HVDC excitation and finally negative polarity HVDC excitation.

- The HTV SR insulator with a USCDof $35 \mathrm{~mm} / \mathrm{kV}$ aged the slowest for HVAC excitation. It aged severely for positive polarity HVDC excitation, followed by negative polarity HVDC excitation.

- The HTV SR insulator (channel 4) had the highest initial peak current for HVAC excitation, followed by positive polarity HVDC excitation and then negative polarity HVDC excitation. However, the highest maximum peak current at the end of the experiment occurred for positive polarity HVDC excitation $\left(48^{\text {th }}\right.$ day), followed by negative polarity HVDC excitation $\left(43^{\text {rd }}\right.$ day) and finally HVAC excitation ( $53^{\text {rd }}$ day).

- The HTV SR insulator had a wettability class of 1 for HVAC excitation, a wettability class of 3 for positive polarity HVDC excitation and a wettability class of 5 for negative polarity HVDC excitation. This comparison showed that the HTV SR insulator (Channel 4) retained its hydrophobicity better for HVAC excitation, followed by positive polarity HVDC excitation and finally negative polarity HVDC.

- In terms of the material aging performance of the porcelain insulator (channel 5), no signs of degradation were visible for HVAC excitation or HVDC excitation. Only minor discoloration of the surface resulted from all three test conditions. 
- The porcelain insulator had the lowest initial peak current for negative polarity HVDC excitation, with a higher initial leakage current for positive polarity HVDC excitation and the highest initial leakage current for HVAC excitation.

- Toward the end of the experiment, the porcelain insulator had the highest peak leakage current for positive polarity HVDC excitation followed by HVAC excitation and finally negative polarity HVDC excitation.

- $\quad$ The porcelain insulator fell within a wettability class of 6 for HVAC excitation and positive and negative polarity HVDC excitation, therefore remaining hydrophilic throughout all the experiments.

- In terms of the material aging performance of the RTV SR coated porcelain insulator (Channel 6), the insulator showed tracking marks and severe erosion for positive polarity HVDC excitation. The RTV SR coated porcelain insulator aged the worst for HVAC stress, followed by positive polarity HVDC excitation and finally negative polarity HVDC excitation.

- $\quad$ Negative polarity HVDC excitation showed the lowest initial peak currents and it took six days before any leakage currents were measureable. It took 53 days for the insulator to reach $220 \mathrm{~mA}$ for HVAC excitation, 31 days for the insulator to fail and reach $724 \mathrm{~mA}$ for positive polarity HVDC excitation and 31 days for the insulator to fail and reach $700 \mathrm{~mA}$ for negative polarity HVDC excitation.

- $\quad$ The RTV SR coated porcelain insulator fell within a wettability class of 3 for HVAC excitation and a wettability class of 4 for both positive and negative polarity HVDC excitation. The RTV SR coated porcelain insulator (Channel 6) retained some of its hydrophobic properties for HVAC excitation and fared slightly worse for positive and negative polarity HVDC excitation. 


\section{REFERENCES}

[1] Vosloo W.L, Macey R.E, de Tourreil C, The Practical Guide to: Outdoor High Voltage Insulators, Crown Publications cc. Johannesburg, July 2004.

[2] Schwardt W.S, Insulator Pollution Monitoring Device: Development, Calibration and Field Evaluation, University of Stellenbosch, December 2004.

[3] Vosloo W.L, A comparison of the Performance of High-Voltage insulator materials in a severely polluted coastal environment thesis, University of Stellenbosch, March 2002.

[4] Holtzhausen J.P, Vosloo W.L, High Voltage Engineering Notes, University of Stellenbosch, 2007.

[5] Mehl E, Design, Commissioning and Evaluation of a Tracking Wheel Tester using Insulator Rod Samples thesis, University of Stellenbosch, October 2006.

[6] Schwardt W.H, Vosloo W.L, Holtzhausen J.P, Risk of Flashover Prediction Based on Site Severity Measurements, SAUPEC 2005, Johannesburg, January 2005.

[7] Schwardt W.H, Holtzhausen J.P, Vosloo W.L, Determination of a Calibration Curve for an Insulator Pollution Monitoring Relay, SAUPEC 2002, Vanderbijlpark, January 2002.

[8] Vosloo W.L, Holtzhausen J.P, The Electric Field of Polluted Insulators, Africon, George, October 2002.

[9] Vosloo W.L, Holtzhausen J.P, The Effect of Thermal Characteristics of Power Line Insulators on Pollution Performance, Africon, George, October 2002.

[10] Vosloo W.L, Holtzhausen J.P, The Prediction of Insulator Leakage Currents from Environmental Data, Africon, George, October 2002.

[11] Pietersen D, Hotzhausen J.P, Vosloo W.L, An Investigation Into the Methodology to Develop an Insulator Pollution Severity Application Map for South Africa, $7^{\text {th }}$ IEEE Africon Conference, Gaborone, Botswana, September 2004.

[12] Schwardt W.H, Holtzhausen J.P, Vosloo W.L, A Comparison Between Measured Leakage Current and Surface Conductivity During Salt Fog Tests, $7^{\text {th }}$ IEEE Africon Conference, Gaborone, Botswana, September 2004. 
[13] Holtzhausen J.P, Vosloo W.L, An Analysis of Leakage Current Waveforms, Measured On-Site, With Reference to Insulator Pollution Flashover Models, Thirteenth International Symposium on High Voltage Engineering, Delft, Netherlands, August 2003.

[14] Holtzhausen J.P, Vosloo W.L, Insulator Pollution and Wetting Processes At a Severe Coastal Site, Thirteenth International Symposium on High Voltage Engineering, Delft, Netherlands, August 2003.

[15] Holtzhausen J.P, Vosloo W.L, Obsevation of Discharge Development and Surface Charges to Evaluate the Performance of Different Outdoor Insulator Materials at a Severe Coastal Site, Thirteenth International Symposium on High Voltage Engineering, Delft, Netherlands, August 2003.

[16] High Voltage Direct Current Transmission, http://en.wikipedia.org/wiki/HVDC

[17] High Voltage Direct Current Transmission, http:www.siemens.com/hvdc

[18] Woodford D.A, HVDC Transmission, Manitoba HVDC Research Centre, Canada, March 1998.

[19] K Folly, EEE490F Power System Analysis Operation and Control Class Notes, UCT, Cape Town, 2006.

[20] Rudervall R, Charpentier J.P, Sharma R, High Voltage Direct Current (HVDC) Transmission Systems Technology Review Paper, March 2000.

[21] Kuffel E, Zaengl W.S, Kuffel J, High Voltage Engineering Fundamentals, Butterworth-Heinemann Publication, 2001.

[22] IEC Publication 60815, Guide for the selection of insulators in respect of polluted conditions, 1986.

[23] IEC 62073, Guidance on the measurement of wettability of insulator surfaces, 2003.

[24] IEC 60507, Artificial pollution tests on high-voltage insulators to be used on a.c. systems, 1991.

[25] Gustavsson T.G, Silicone Rubber Insulators: Impacts of Material Formulation in Coastal Environment, Chalmers University of Technology Göteborg, Sweden, April 2002.

[26] Pietersen D, The development of a methodology to compile insulator pollution severity application map for South Africa, University of Stellenbosch, April 2005. 
[27] IEC 61302, Electrical insulating materials - Method to evaluate the resistance to tracking and erosion - Rotating wheel dip test, 1995.

[28] Telcon HTP 25 Specification, http://www.arthurbehrens.biz/data/stromwandler/HTP25.pdf

[29] Nampower Tender No:NPWR/2006/22, Caprivi Link Interconnector: Converter Stations Project, Namibia, Nampower, 2006.

[30] Ma W.M, etal, Preliminary Recommendations on the Suitable Shed Profile for HVDC Station Insulators with Silicone Rubber Housing, EPRI

[31] Wikström D, Öhlen C, Composite Insulation for Reliability Centered Design of compact HVAC \& HVDC, STRI, 2004.

[32] Chandrasekar S, Sarathi R, Danikas M.G, Analysis of surface degradation of silicone rubber insulation due to tracking under different voltage profiles, May 2006.

[33] Seifert J. M, Bärsch, Design Evaluation of Silicone Rubber Composite Insulators under the Aspect of Surface Pollution Stress, XVth International Symposium on High Voltage Engineering, University of Ljubljana, Slovenia, August 2007.

[34] Jiang X, etal, Comparison of DC Pollution Flashover Performances of Various Types of Porcelain, Glass, and Composite Insulators, IEEE Transactions on Power Delivery, Vol 23, No 2, April 2008.

[35] Pargamin L, Huc J, Tartier S, Considerations on the choice of the insulators for HVDC overhead lines, International Conference on Large High Voltage Electric Systems, France, 1984.

[36] Zhiyi S, etal, Natural contamination performances on insulators under DC stress and the pollution deposit density ration of $D C$ to $A C$, China Electric ;Power Research Institute, Beijing, China, 2007.

[37] Agrawal L.N, Kapur A.K, Sachdeva M.L, Insulation Performance of $\pm 500 \mathrm{kV}$ HVDC Rihand - Dadri Line in India, Powergrid Corporation of India Ltd, India, 2000 .

[38] Jun Z, etal, Study on the Various Pollution Performance of DC post Insulators under High Altitude area, Tsinghua University, China, August 2005.

[39] Zhiyi S, etal, Outdoor Insulation Selection Method of HVDC lines, Tsinghua University, China, August 2005. 
[40] IEC 62217, Polymeric insulators for indoor and outdoor use with a nominal voltage $>1000 \mathrm{~V}$ - General definitions, test methods and acceptance area, 2005.

[41] Galvanic Corrosion

http://en.wikipedia.org/wiki/Galvanic _corrosion 


\section{Appendix A}

\section{A1: Design of the protection circuit}

Fig A.1 shows the protection circuit that was used in the experiment. There protection circuit was described in detail in Chapter: 3. However, the simulation program, as well as the calculation of how the resistor values were chosen are given in this section.

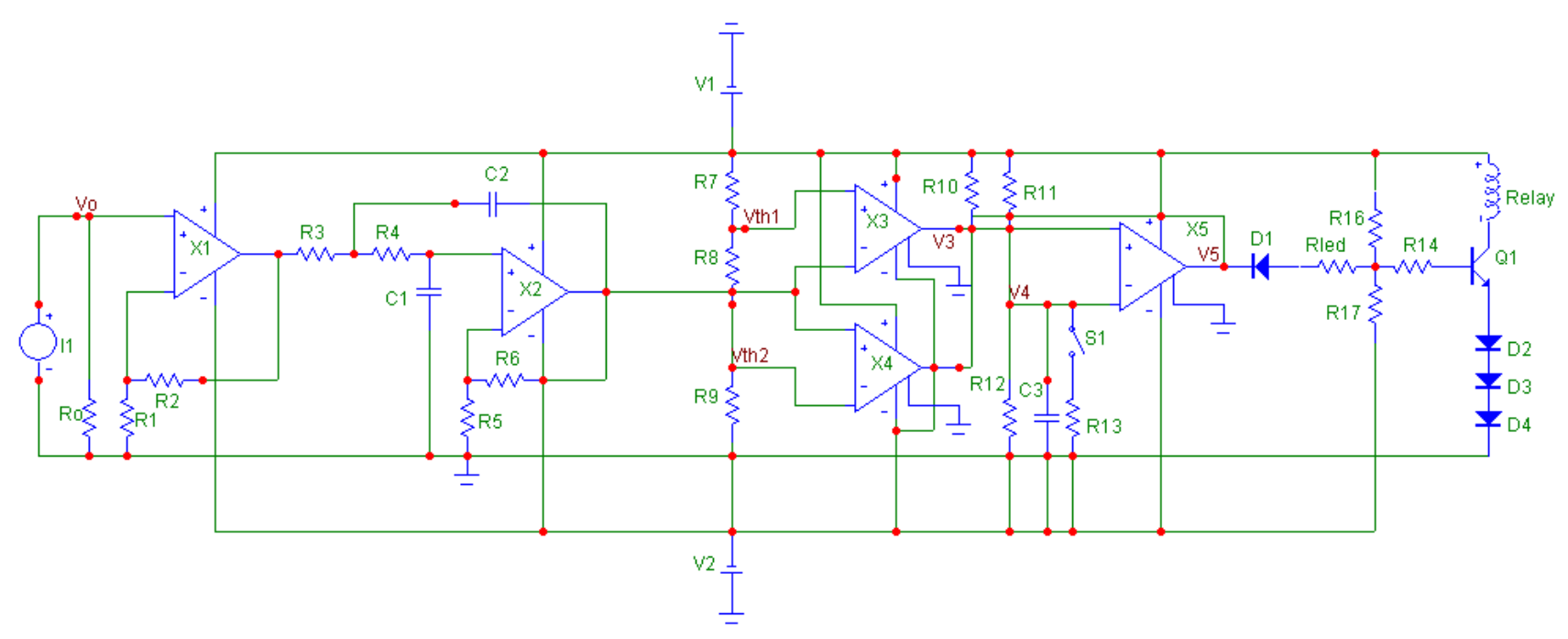

Figure A. 1: The protection circuit showing the electronic circuit.

\section{Non - Inverting Buffer/Amplifier}

The Telcon HTP 25 was used as a current transformer. It is a closed loop Hall Effect Current Transformer that provides an output current into an external load resistance. It has a ratio of 1000:1. The specification for the Telcon HTP 25 is given in below.

\section{The Telcon HTP 25 has the following features and benefits:}

- $\quad$ High accuracy.

- Galvanic isolation between the primary and secondary circuit.

- $\quad$ High Reliability.

- $\quad$ Fast Response.

- Wide dynamic range.

The leakage current of each of the rod specimens is measured individually using halleffect current transducers. Each current signal is fed through a separate buffer 
amplifier, low pass filter, level comparator and latching circuit. All channels share a common relay driver or tripping circuit.

The non-inverting buffer amplifies the input voltage from the current transducer and reflects a high input impedance to limit the loading on the transducer output.

$$
\mathrm{S}_{\text {base3థ }}=100 \mathrm{kVA} ; \mathrm{V}_{\text {baseLL1 }}=22 \mathrm{kV} ; \mathrm{Z}_{p u}=0.0429 \text { (Impedance voltage) }
$$

$\mathrm{Z}_{\text {basel }}=\frac{\left(V_{\text {baseLL1 }}\right)^{2}}{S_{\text {base3 } \Phi}}$

$$
\begin{aligned}
& =\frac{(22000 \mathrm{~V})^{2}}{100000 V A} \\
& =4840 \Omega
\end{aligned}
$$

$\mathrm{Z}_{\text {actual }}=\mathrm{Z}_{p u} \times \mathrm{Z}_{\text {base }}$

$$
=0.0429_{p u} \times 4840 \Omega
$$$$
=207.636 \Omega
$$

$$
\begin{aligned}
I_{s c 1} & =\frac{V_{L N 1}}{Z_{\text {actual }}} \\
& =\frac{(22000 / \sqrt{3} V)}{207.636 \Omega} \\
& =61.17 \mathrm{~A}
\end{aligned}
$$

The short circuit current on the secondary of T3 is calculated as follows, given the fact that $\mathrm{V}_{L L 2}=10 \mathrm{kV}$ :

$$
\begin{aligned}
I_{s c 2} & =\frac{V_{L N 2}}{Z_{\text {actual }}} \\
& =\frac{(10000 / \sqrt{3} V)}{207.636 \Omega} \\
& =27.806 \mathrm{~A} \\
& =27.81 \mathrm{~A}
\end{aligned}
$$


The calculation shows that the transformer has a flashover current of 27.806A.This is why the Telcon with a nominal primary current of $25 \mathrm{~A}$ and a Linearity Limit of $\pm 55 \mathrm{~A}$ peak was chosen. Telcon HTP 25 has a nominal power supply of $\pm 15 \mathrm{~V}$ with $150 \Omega$ minimum load resistances. It has a $3 \mathrm{kV}$ proof stress voltage. With the turns ratio being 1000:1, you can calculate the secondary short circuit current if the primary short circuit current $\left(\mathrm{I}_{p s c}\right)$ is $28 \mathrm{~A}$. Therefore the secondary short circuit current $\left(\mathrm{I}_{s s c}\right)$ works out as $28 \mathrm{~mA}$. Whereby a threshold r.m.s leakage current $\left(\mathrm{I}_{p t h}\right)$ of $300 \mathrm{~mA}$ will give a secondary threshold current $\left(\mathrm{I}_{s t h}\right)$ of $0.3 \mathrm{~mA}$. Because $\mathrm{I}_{p t h}$ was so low, 5 turns were added on the primary side to increase the leakage current.

$$
\begin{aligned}
I_{\text {sthnew }} & =\frac{\left(I_{p t h} \times 31\right)}{1000} \\
& =9.3 \mathrm{~mA} \\
I_{p \text { sthnew }} & =9.3 \mathrm{~mA} \times \sqrt{2}=13.15 \mathrm{~mA} \\
I_{\text {sscnew }} & =\frac{\left(I_{p s c} \times 31\right)}{1000} \\
= & 0.868 \mathrm{~A}
\end{aligned}
$$

With $\mathrm{V}_{t h 1}=10 \mathrm{~V}$ at the window comparator and the non-inverting buffer/amplifier with a gain of 2 will give the following value for $\mathrm{R}_{o}$ :

$$
\mathrm{V}_{o}=\frac{V_{t h 1}}{4}
$$

$$
\begin{aligned}
& =2.5 \mathrm{~V} \\
\mathrm{R}_{o} & =\frac{V_{o}}{I_{\text {sthnew }}} \\
& =\frac{2.5 \mathrm{~V}}{13.15 m A} \\
& =197 \Omega
\end{aligned}
$$


When dealing with short circuit conditions:

$$
\begin{aligned}
\mathrm{V}_{0} & =\mathrm{I}_{\text {sscnew }} \times \mathrm{R}_{o} \\
& =0.868 \mathrm{~A} \times 200 \Omega \\
& =173.6 \mathrm{~V}
\end{aligned}
$$

The non-inverting buffer consists of the following components. It is made of a LF411 opamp with dual power supply of \pm 15 Volts. The non-inverting input has a current source input with resistance of $200 \Omega$. The inverting input is connected to resistances $\mathrm{R} 1$ and R2 with a voltage gain of 2 .

\section{H-spice simulation program Non-Inverting Amplifier}

\section{A NONINVERTING AMPLIFIER}

\section{.OPTIONS LIST NODE POST}

vinput $10 \operatorname{SIN}(02.55000)$

R2 $324.7 k$

R1 $204.7 \mathrm{k}$

e1 $3012999 \mathrm{meg}$

.TRAN $.5 \mathrm{~m} 25 \mathrm{~m}$

.PLOT TRAN V(1,0) V(3,0)

END 


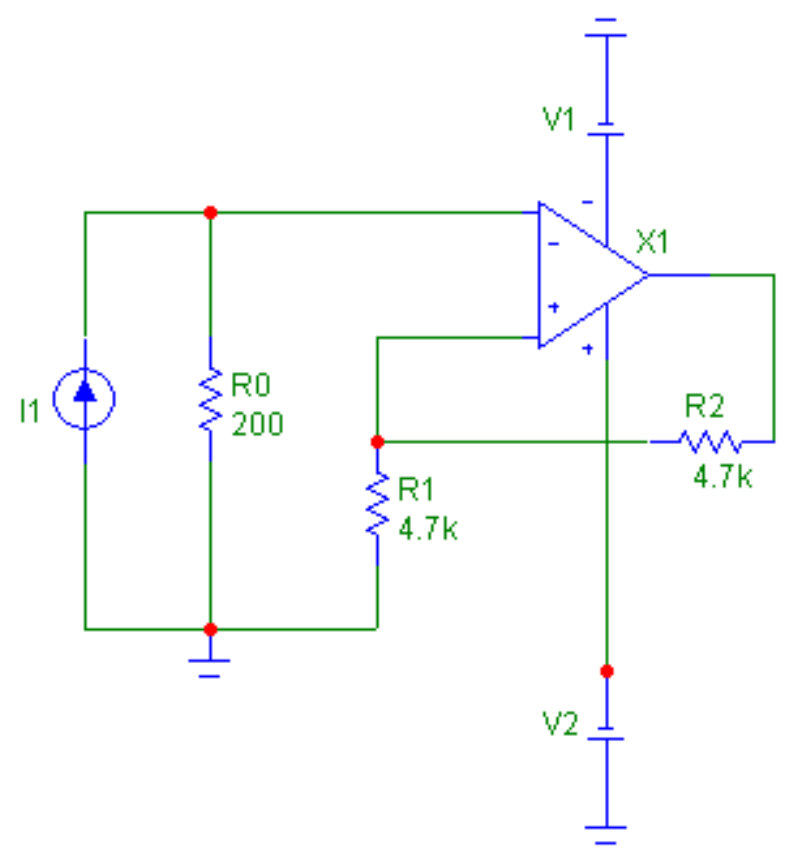

Figure A. 2: The Non-Inverting Amplifier Circuit.

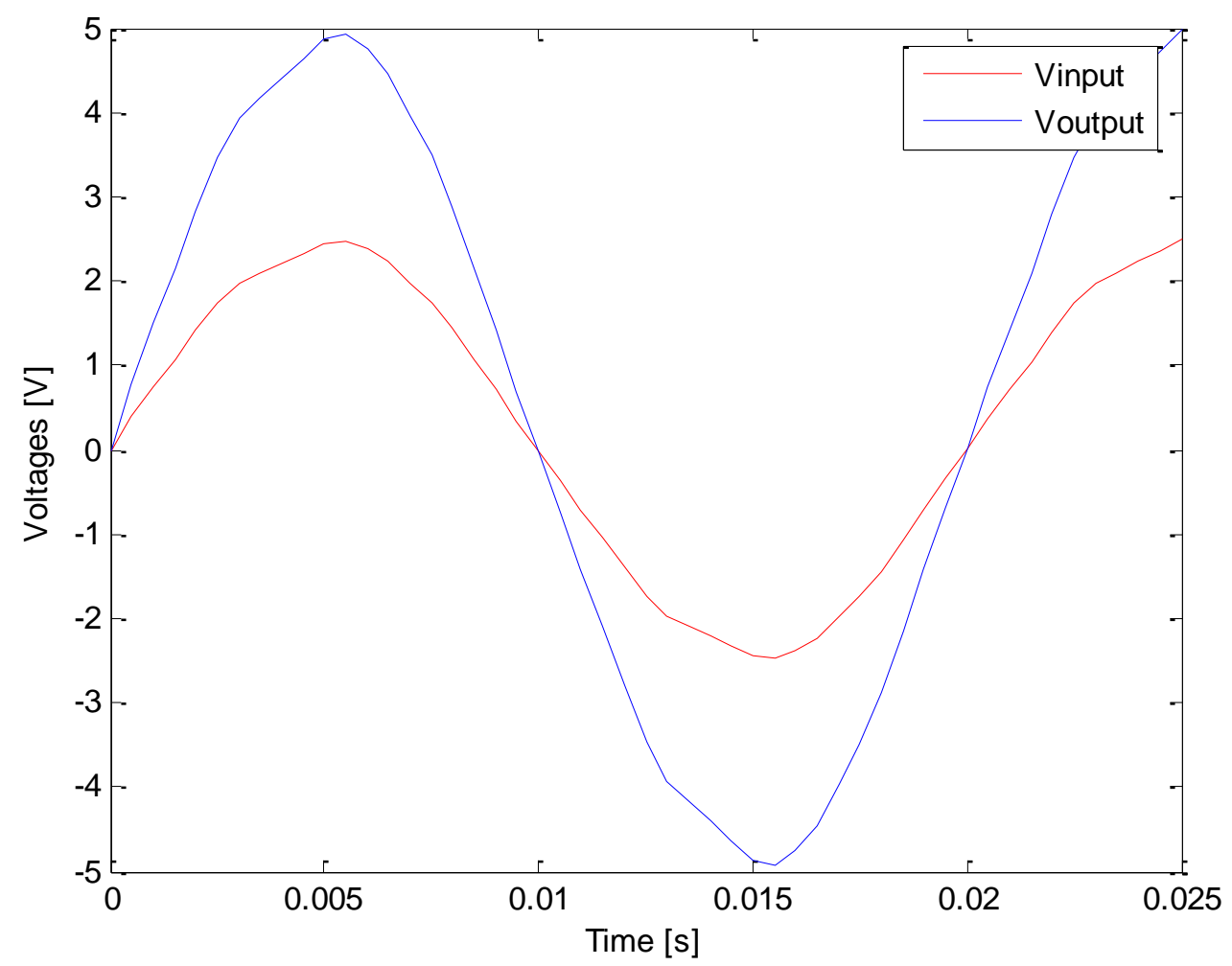

Figure A. 3: The Non-Inverting Amplifier with a gain of 2. 
The Current Transformer was modeled by a current source in series with a $200 \Omega$ resistor. The value of the current source will be $1.5 \mathrm{~mA}$, which will give a voltage input of $5 \mathrm{~V}$.

\section{Sallen-Key $2^{\text {nd }}$ order Low Pass Filter}

Fig. A. 4 shows the electronic circuit of the Sallen-Key $2^{\text {nd }}$ order Low Pass Filter. This section will also give the simulation program.

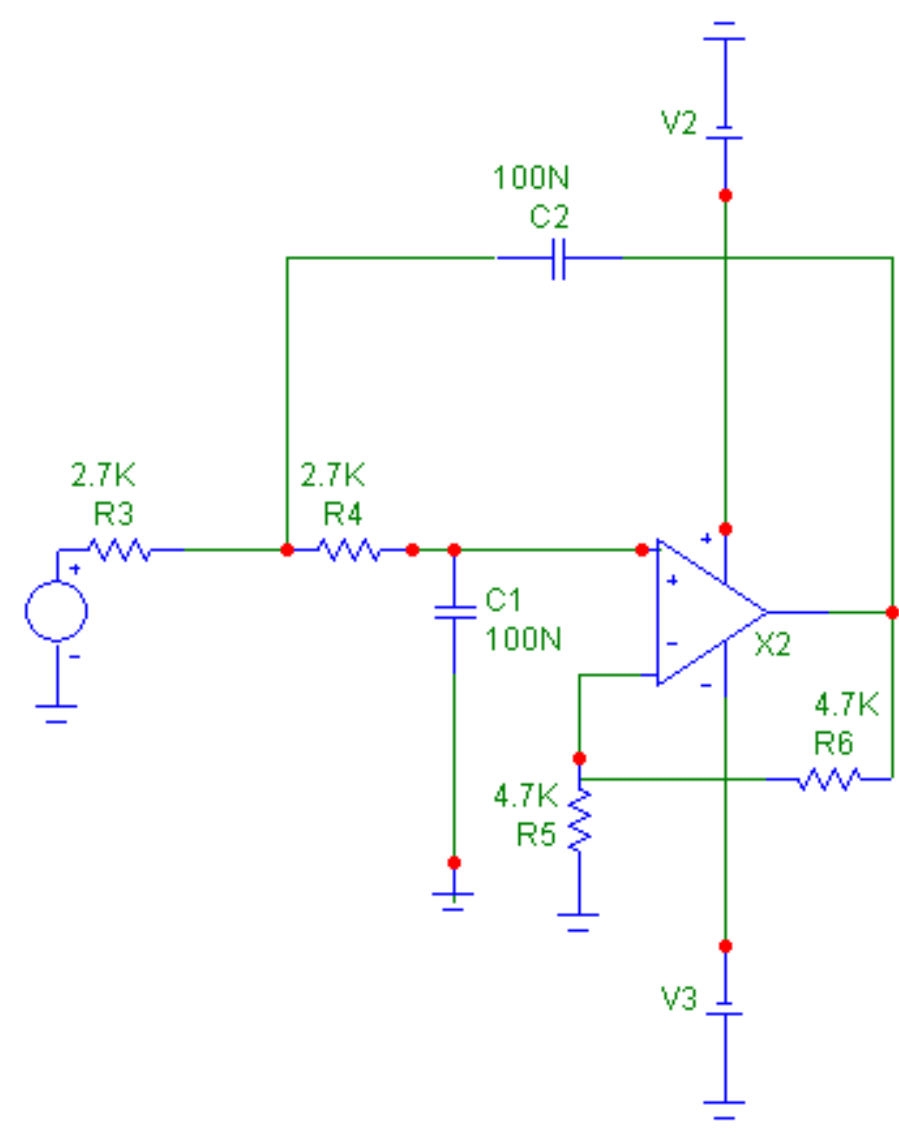

Figure A. 4: The electronic circuit of the Sallen-Key 2nd order Low Pass Filter.

The Sallen-Key Second Order Low Pass Filter Simulation

OPSALKEY1.CIR - OPAMP SALLEN-KEY LOW-PASS FILTER

* 2ND-ORDER BUTTERWORTH

$*$

$\begin{array}{lllll}\mathrm{VS} & 1 & 0 & \mathrm{AC} & 5\end{array}$

R1 $1 \quad 2 \quad 2.7 \mathrm{~K}$ 
$\begin{array}{llll}\mathrm{R} 2 & 2 & 3 & 2.7 \mathrm{~K}\end{array}$

$\begin{array}{llll}\mathrm{C} 1 & 2 & 5 & 100 \mathrm{NF}\end{array}$

$\begin{array}{llll}\mathrm{C} 2 & 3 & 0 & 100 \mathrm{NF}\end{array}$

$*$

* UNITY GAIN AMPLIFIER, RA=OPEN, RB=SHORT

RA $4 \quad 0 \quad 4.7 \mathrm{~K}$

$\begin{array}{llll}\mathrm{RB} & 4 & 5 & 4.7 \mathrm{~K}\end{array}$

$\begin{array}{llll}\text { XOP } & 34 & 5 & \text { OPAMP1 }\end{array}$

$*$

* SINGLE RC FILTER FOR COMPARISON

*R10 $11 \quad 10 \quad 15.9 \mathrm{~K}$

${ }^{*} \mathrm{C} 10 \quad 10 \quad 0 \quad 1000 \mathrm{PF}$

$*$

* OPAMP MACRO MODEL, SINGLE-POLE

* connections: non-inverting input

* $\quad$ inverting input

$* \quad \quad||$ output

* $\quad$ | | |

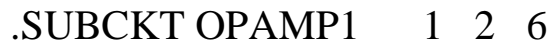

* INPUT IMPEDANCE

RIN $1 \quad 2 \quad 10 M E G$

* DC GAIN (100K) AND POLE 1 (100HZ)

$*$ GBWP $=10 \mathrm{MHz}$

EGAIN $30 \quad 12 \quad 100 \mathrm{~K}$

$\begin{array}{llll}\mathrm{RP} 1 & 3 & 4 & 1 \mathrm{~K}\end{array}$

$\begin{array}{llll}\mathrm{CP} 1 & 4 & 0 & 1.5915 \mathrm{UF}\end{array}$ 
* OUTPUT BUFFER AND RESISTANCE

EBUFFER $50 \quad 40 \quad 1$

$\begin{array}{llll}\text { ROUT } & 5 & 6 & 10\end{array}$

ENDS

$*$

* ANALYSIS

.AC DEC $101010 \mathrm{~K}$

* VIEW RESULTS

.PLOT AC V(5)

.PROBE

END

\section{Deriving the $2^{\text {nd }}$ order Sallen-Key Low Pass Filter}

The Sallen-Key $2^{\text {nd }}$ order Low Pass filter is chosen because of its flexibility and ease of design. The equation of the Sallen-Key $2^{\text {nd }}$ order Low Pass will be derived in the following way.

Important things to note:

- The input voltage of the amplifier will be equal to the output voltage divided by the gain $\mathrm{K}$.

- The gain $\mathrm{K}$ represents a non-inverting amplifier. There is an input signal on your left, which is amplified by your gain $\mathrm{K}$ and outputted to the right.

- The output is a perfect voltage source since the input of the amplifier will not draw any current.

- The impedance of the capacitance is $\mathrm{X}_{c}=\frac{1}{S x C}$

The standard second order filter equation is in the following form: 


$$
\frac{V_{\text {out }}}{V_{\text {in }}}=\mathrm{K} \times \frac{\left(W_{o}\right)^{2}}{s+s \frac{w_{o}}{Q}+\left(W_{o}\right)^{2}}
$$

Where: $W_{o}$ is the cut - off frequency of the filter.

$\mathrm{Q}$ is a factor that determined the shape of the curve.

The damping factor is the inverse of $\mathrm{Q}$.

- At low frequencies Equation [3.1] approaches $\mathrm{K}$ since the $\mathrm{S}$ terms will be so small, almost approaching 0 .

- $\quad$ At high frequencies equation [3.1] will approach zero since the $S$ terms will become predominantly large.

From the Current across each node, you will get the following equations:

$$
\begin{aligned}
& \frac{V_{\text {in }}-V_{1}}{R_{1}}+\frac{V_{\text {out }}-V_{1}}{\frac{1}{s x C_{2}}}+\frac{\frac{V_{\text {out }}}{K}-V_{1}}{R_{2}}=0 \\
& \frac{V_{1}-\frac{V_{\text {out }}}{K}}{R_{2}}-\frac{\frac{V_{\text {out }}}{K}}{\frac{1}{s x C_{2}}}=0
\end{aligned}
$$

Solving for $\mathrm{V}_{1}$ in equation [A.9] and substituting it in equation [A.10] will give you the final equation for the $2^{\text {nd }}$ order Sallen-Key Low Pass filter.

$$
\frac{V_{\text {out }}}{V_{\text {in }}}=K \times \frac{\frac{1}{R_{1} \times R_{2} \times R_{3} \times R_{4}}}{s+s\left[\frac{1}{R_{1} \times C_{1}}+\frac{1}{R_{2} \times C_{1}}+\frac{1}{R_{2} \times C_{2}}(1-K)\right]+\frac{1}{R_{1} \times R_{2} \times C_{1} \times C_{2}}}
$$

We can see from equation [A.8] and [A.11] that the cut-off frequency is given by:

$$
\mathrm{W}_{o}=\frac{1}{\sqrt{R_{1} \times R_{2} \times C_{1} \times C_{2}}}
$$

If the middle term of equation [A.9] is taken and divided by the cut-off frequency, then the damping factor is obtained. The inverse of the damping factor is known as the $\mathrm{Q}$ factor. It is given by the following formula: 


$$
\mathrm{Q}=\frac{1}{\sqrt{\frac{R_{2} \times C_{2}}{R_{1} \times C_{1}}}+\sqrt{\frac{R_{1} \times C_{2}}{R_{2} \times C_{1}}}+(1-K) \sqrt{\frac{R_{1} \times C_{1}}{R_{2} \times C_{2}}}}
$$

Equation [A.11] and [A.12] shows that there are five variables. But the filter can be further simplified by making a few assumptions. The assumptions are that $\mathrm{R}_{1}=\mathrm{R}_{2}=\mathrm{R}$ and $C{ }_{1}=C_{2}=C$. The gain $\mathrm{K}=2$ is used.

With the assumptions made equation [A.12] and [A.13] will simplify to:

$$
\begin{aligned}
& \mathrm{W}_{o}=\frac{1}{R C} \\
& \mathrm{Q}=\frac{1}{3-K}
\end{aligned}
$$

The gain $\mathrm{K}$ is given by the following equation:

$$
\begin{aligned}
\mathrm{K} & =1+\frac{R_{6}}{R_{5}} \\
& =1+\frac{4 k 7}{4 k 7} \\
& =2
\end{aligned}
$$

The values of the Resistors are $R_{6}=R_{5}=4 k 7 \Omega$ and $R_{4}=R_{3}=2 k 7 \Omega$. The value of the capacitor is $\mathrm{C}_{1}=C_{2}=0.1 \mu \mathrm{F}$.

The cut-off frequency for this particular filter is:

$$
\begin{aligned}
\mathrm{F}_{c} & =\frac{1}{2 \pi R C} \\
& =\frac{1}{2 \pi\left(2.7 \times 10^{3}\right)\left(0.1 \times 10^{-6}\right)} \\
& =589.46 \mathrm{~Hz}
\end{aligned}
$$




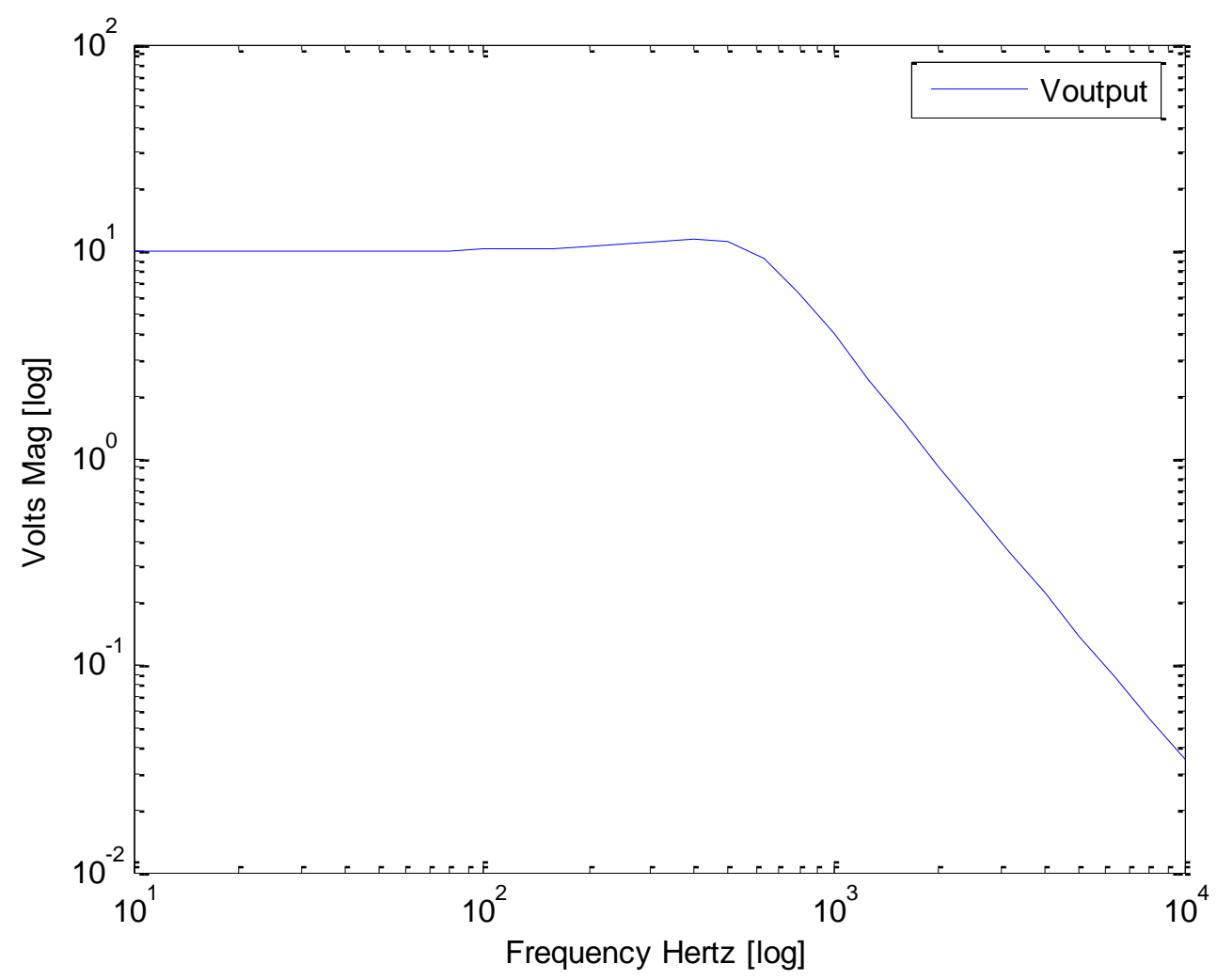

Figure A. 5: The output of the Sallen-key 2nd order Low Pass filter.

\section{The Window Comparator}

The LM311 comparator was used. The LM311 Comparator was chosen because of its unique features. The LM311 is a fast comparator which can amplify any small signal with input noise. This is done without any oscillations and requirements for positive feedback. 


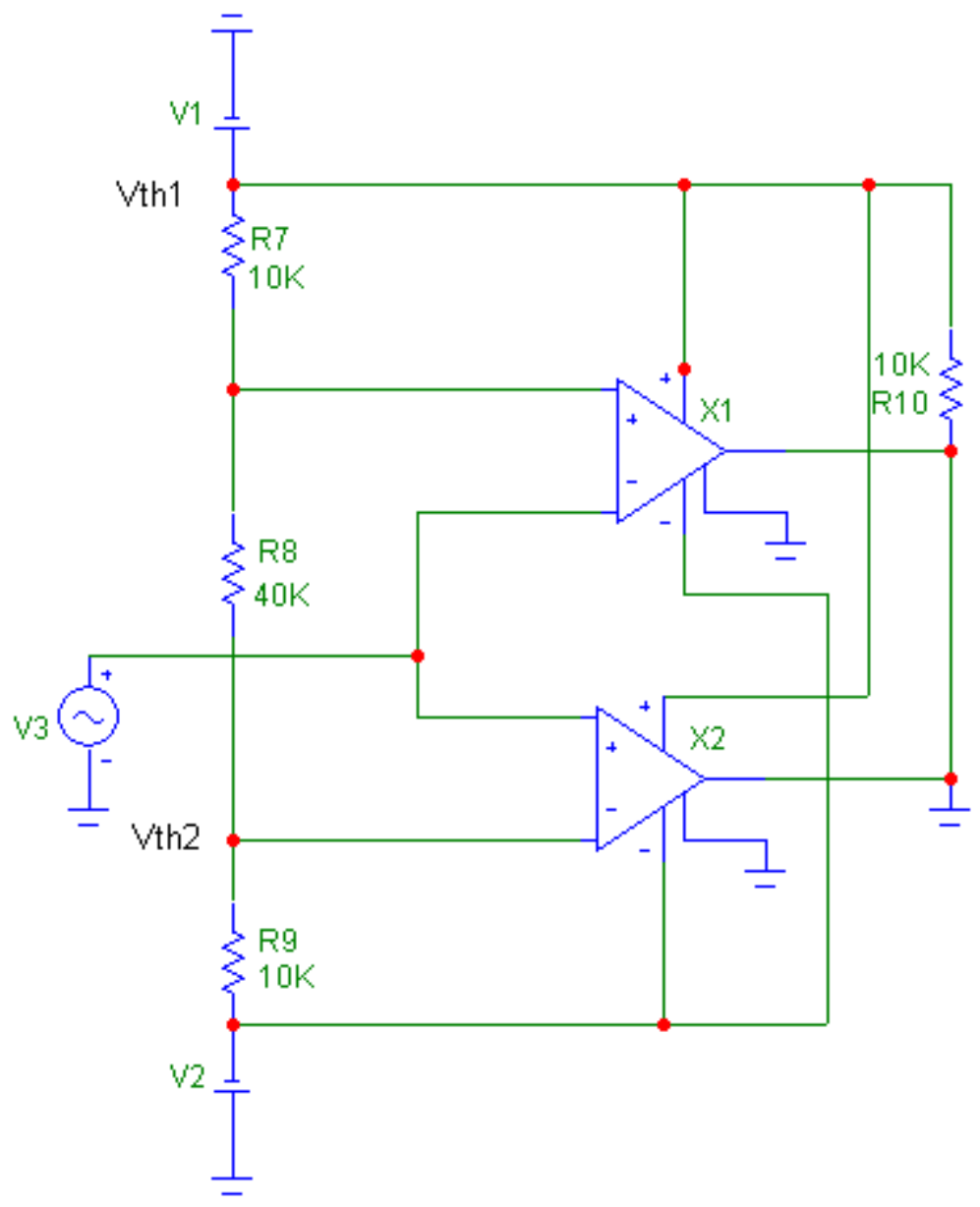

Figure A. 6: The Electronic Circuit showing the Window Comparator.

\section{Deriving the Resistor Values}

Vth1 is given as $10 \mathrm{~V}$, whereas Vth2 is given as $-10 \mathrm{~V}$. The Voltage across R7 then becomes:

$$
\begin{aligned}
\mathrm{V}_{7} & =\mathrm{Vcc}-\mathrm{Vth} 1 \\
& =15 \mathrm{~V}-10 \mathrm{~V} \\
= & 5 \mathrm{~V}
\end{aligned}
$$

The Voltage $\left(\mathrm{V}_{9}\right)$ across $\mathrm{R}_{9}$ is equal to $\mathrm{V}_{7}$. Therefore the voltage across $\mathrm{R}_{8}$ becomes:

$$
\begin{aligned}
\mathrm{V}_{8} & =2 \times \mathrm{Vcc}-\mathrm{V}_{7}-\mathrm{V}_{9} \\
& =30 \mathrm{~V}-5 \mathrm{~V}-5 \mathrm{~V} \\
& =20 \mathrm{~V}
\end{aligned}
$$

There resistor values are then obtained from the following formula, assuming that the current flowing through the resistors is the same: 
$\frac{V_{8}}{R_{8}}=\frac{V_{7}}{R_{7}}=\frac{V_{9}}{R_{9}}$

This current is chosen to be $1 \mathrm{~mA}$ :

$\frac{R_{7}}{R_{8}}=\frac{V_{7}}{V_{8}}=\frac{5}{20}=0.25$

Taking into consideration the fact that $\mathrm{R}_{7}=\mathrm{R}_{9}=\mathrm{R}_{8}(0.25) . \mathrm{R}_{7}$ is chosen to be $10 \mathrm{k} \Omega$, therefore $\mathrm{R}_{9}$ also becomes $10 \mathrm{k} \Omega$. $\mathrm{R}_{8}$ becomes $40 \mathrm{k} \Omega$ from equation [A.20].

\section{Simulation Program for the Window Comparator}

A WINDOW COMPARATOR

.OPTIONS LIST NODE POST

Vin $50 \operatorname{SIN}(0125000)$

R7 $1210 \mathrm{~K}$

R8 $2340 \mathrm{~K}$

R9 $3410 \mathrm{~K}$

VS1 1015

vs2 0415

R10 71 10K

X1251470 LM311

X2531470 LM311

* LM311 VOLTAGE COMPARATOR "MACROMODEL" SUBCIRCUIT

* CREATED USING PARTS VERSION 4.03 ON 03/07/90 AT 08:15

$* \operatorname{REV}(\mathrm{N} / \mathrm{A})$

* CONNECTIONS: NON-INVERTING INPUT

* $\quad$ INVERTING INPUT

* | | POSITIVE POWER SUPPLY 


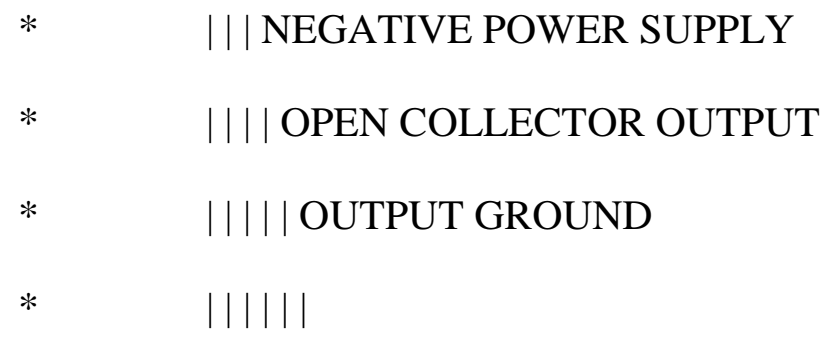

\section{.SUBCKT LM311 123456}

$*$

F1 93 V1 1

IEE 37 DC 100.0E-6

VI1 211 DC .45

VI2 222 DC .45

Q1 9217 QIN

Q2 8227 QIN

Q3 $98 \quad 4$ QMO

Q4 8884 QMI

.MODEL QIN PNP(IS=800.0E-18 BF=500)

.MODEL QMI NPN(IS=800.0E-18 BF=1002)

.MODEL QMO NPN(IS=800.0E-18 BF=1000 CJC=1E-15 TR=102.5E-9)

E1 $10 \begin{array}{llll}10 & 6 & 9 & 4\end{array}$

V1 1011 DC 0

Q5 5116 QOC

.MODEL QOC NPN(IS=800.0E-18 $\quad$ BF=103.5E3 $\quad$ CJC=1E-15 TF=11.60E-12 $\mathrm{TR}=48.19 \mathrm{E}-9$ )

DP $43 \mathrm{DX}$

RP $3 \quad 46.667 \mathrm{E} 3$

.MODEL DX D(IS=800.0E-18) 


\section{.ENDS}

.TRAN $.5 \mathrm{~m} 25 \mathrm{~m}$

.PLOT TRAN V(2) V(5) V(3) v(7)

END

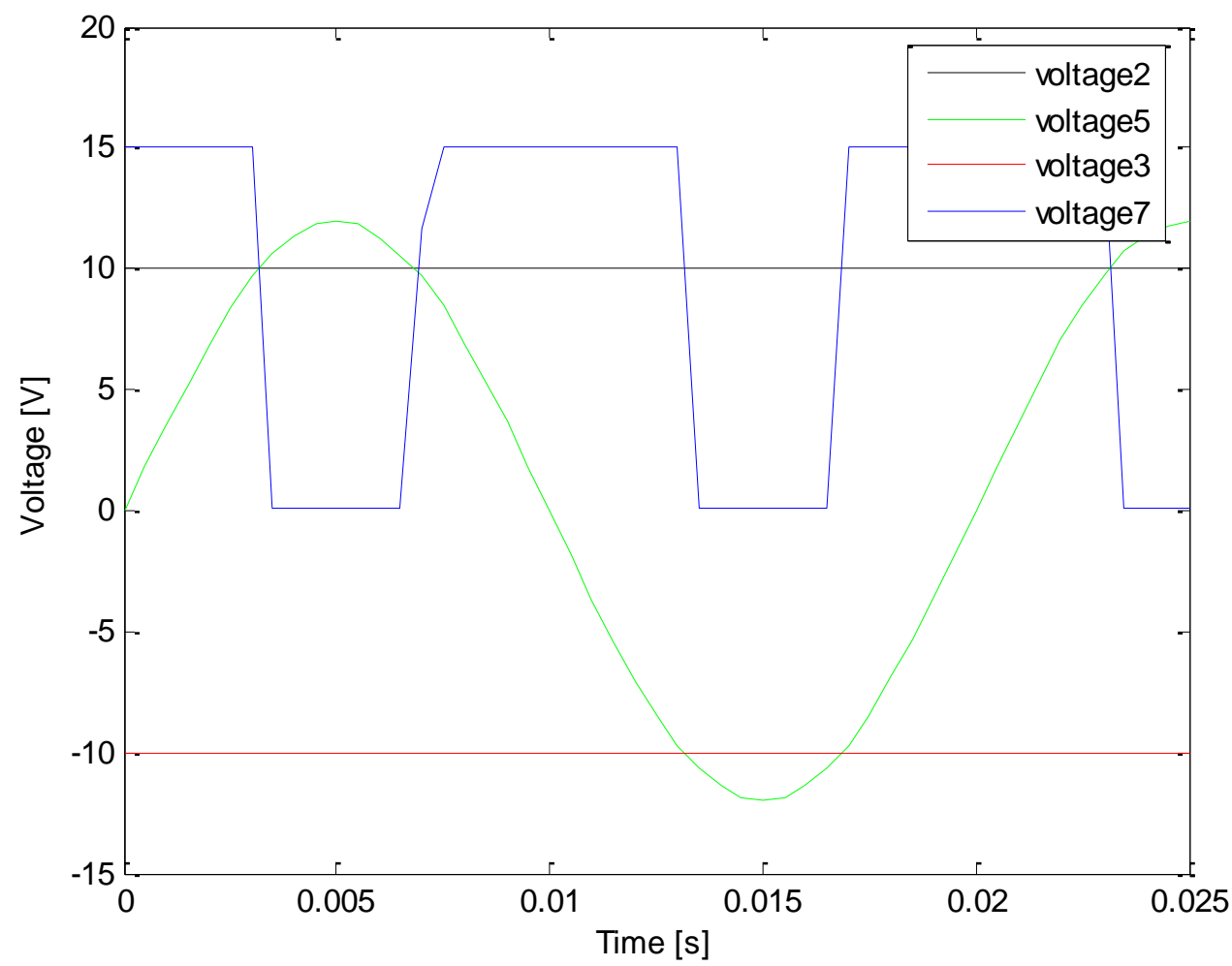

Figure A. 7: The operation of the Window Comparator.

\section{The Latching Circuit}

There latching circuit and the simulation program are given below. 


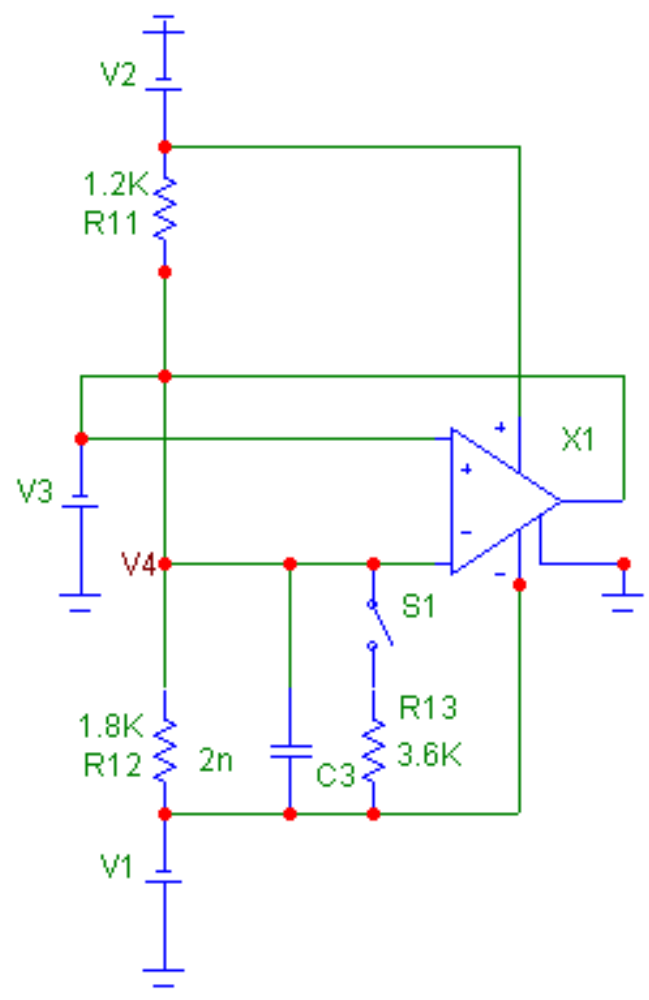

Figure A. 8: The electronic circuit showing the latching circuit.

\section{Derivation of Component Values}

During normal operation, $\mathrm{V}_{4}$ is chosen to be $3 \mathrm{~V}$. The following formal is applied to get the resistor values:

$\frac{V_{c c}-V_{4}}{R_{11}}+\frac{V_{c c-}-V_{4}}{R_{12}}=0$

$\frac{15 V-3 V}{R_{11}}=\frac{15 V+3 V}{R_{12}}$

If $R_{12}$ is chosen to be $1.8 \mathrm{k} \Omega$, then $R_{11}$ becomes $1.2 \mathrm{k} \Omega$.

To reset the Latching Circuit, $\mathrm{V}_{4}$ is chosen as $0 \mathrm{~V}$ and $\left(\mathrm{R}_{13} \| \mathrm{R}_{12}\right)=\mathrm{R}_{11}=1.2 \mathrm{k} \Omega$ :

$\frac{R_{12} \times R_{13}}{R_{12}+R_{13}}=\mathrm{R}_{11}$

The equation is solved for $\mathrm{R}_{13}$, and it becomes:

$\mathrm{R}_{13}=\frac{R_{12} \times R_{11}}{R_{12}-R_{11}}$ 


$$
\begin{aligned}
& =\frac{1.8 \mathrm{k} \Omega \times 1.2 \mathrm{k} \Omega}{1.8 \mathrm{k} \Omega-1.2 \mathrm{k} \Omega} \\
& =3.6 \mathrm{k} \Omega
\end{aligned}
$$

H-Spice Simulation Program for the Latching Circuit

\begin{abstract}
A WINDOW COMPARATOR
.OPTIONS LIST NODE POST
\end{abstract}

Vin $50 \mathrm{SIN}(0125000)$

R7 12 10K

R8 $2340 \mathrm{~K}$

R9 34 10K

VS1 1015

vs2 0415

R10 $7110 \mathrm{~K}$

X1251470 LM311

X2531470 LM311

$*$

*LATCHING CIRCUIT

R11 $181.2 \mathrm{~K}$

R12 $841.8 \mathrm{~K}$

C3 $842 \mathrm{NF}$

*R13 $843.6 \mathrm{~K}$

X378 1470 LM311

* LM311 VOLTAGE COMPARATOR "MACROMODEL" SUBCIRCUIT

* CREATED USING PARTS VERSION 4.03 ON 03/07/90 AT 08:15

* REV (N/A) 
* CONNECTIONS: NON-INVERTING INPUT

* $\quad$ INVERTING INPUT

* | | POSITIVE POWER SUPPLY

* $\quad$ || NEGATIVE POWER SUPPLY

* $\quad$ || || OPEN COLLECTOR OUTPUT

* $\quad$ || || $\mid$ OUTPUT GROUND

* $\quad\|\| \|$

.SUBCKT LM311 123456

$*$

F1 93 V1 1

IEE 37 DC 100.0E-6

VI1 211 DC .45

VI2 222 DC .45

Q1 9217 QIN

Q2 8227 QIN

Q3 984 QMO

Q4 8884 QMI

.MODEL QIN PNP(IS=800.0E-18 BF=500)

.MODEL QMI NPN(IS=800.0E-18 BF=1002)

.MODEL QMO NPN(IS=800.0E-18 BF=1000 CJC=1E-15 TR=102.5E-9)

E1 110606104

V1 1011 DC 0

Q5 5116 QOC

.MODEL QOC NPN(IS=800.0E-18 $\quad$ BF=103.5E3 $\quad$ CJC=1E-15 TF=11.60E-12 $\mathrm{TR}=48.19 \mathrm{E}-9$ )

DP $43 \mathrm{DX}$ 
RP $346.667 \mathrm{E} 3$

.MODEL DX D(IS=800.0E-18)

$*$

ENDS

TRAN $.5 \mathrm{~m} 25 \mathrm{~m}$

.PLOT TRAN V(2) V(5) V(3) v(7) V(8)

END

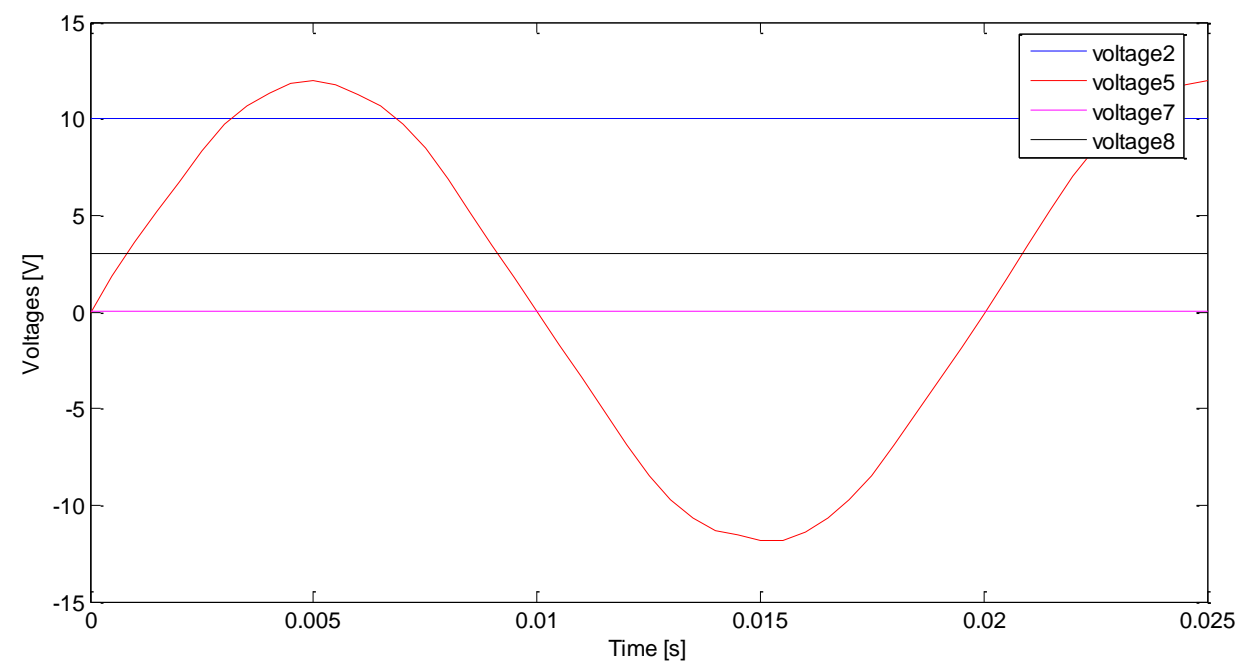

Figure A. 9: The Latching circuit before the reset button is pressed.

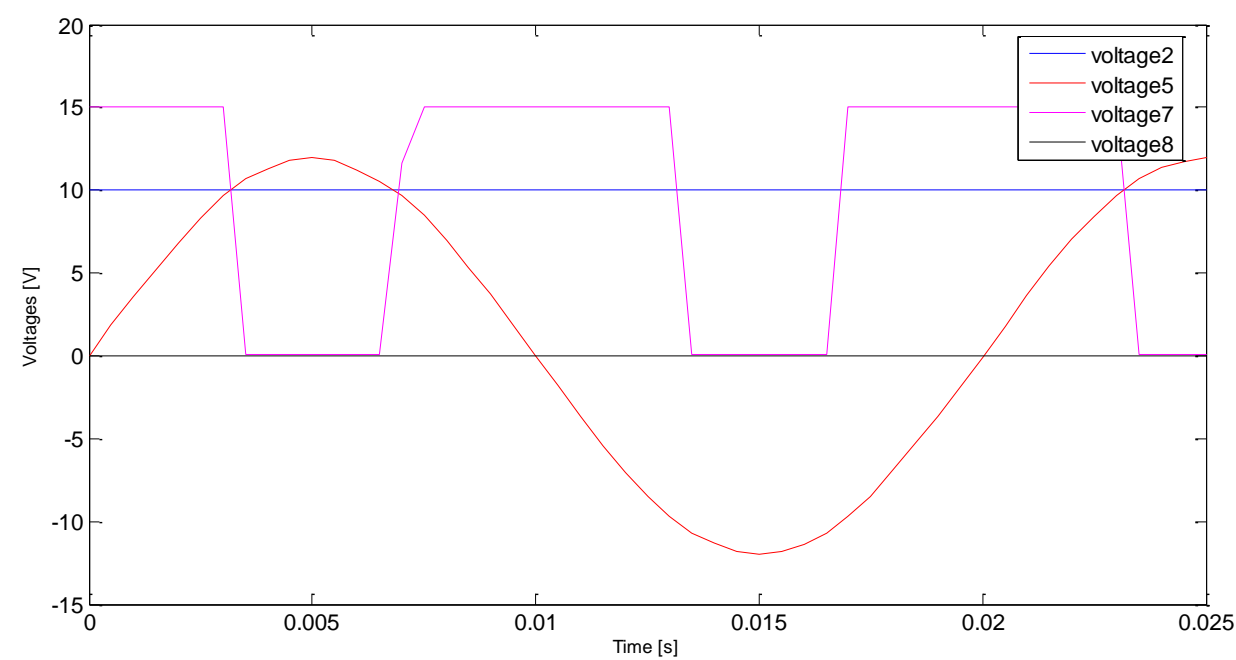

Figure A. 10: The Latching Circuit when the reset button is pressed. 


\section{The Relay Driver}

The circuit diagram for the relay driver is given below. Furthermore, the resistor values and how they were obtained will be given. The operation of the relay driver, and there part it plays is also outlined in the protection circuit.

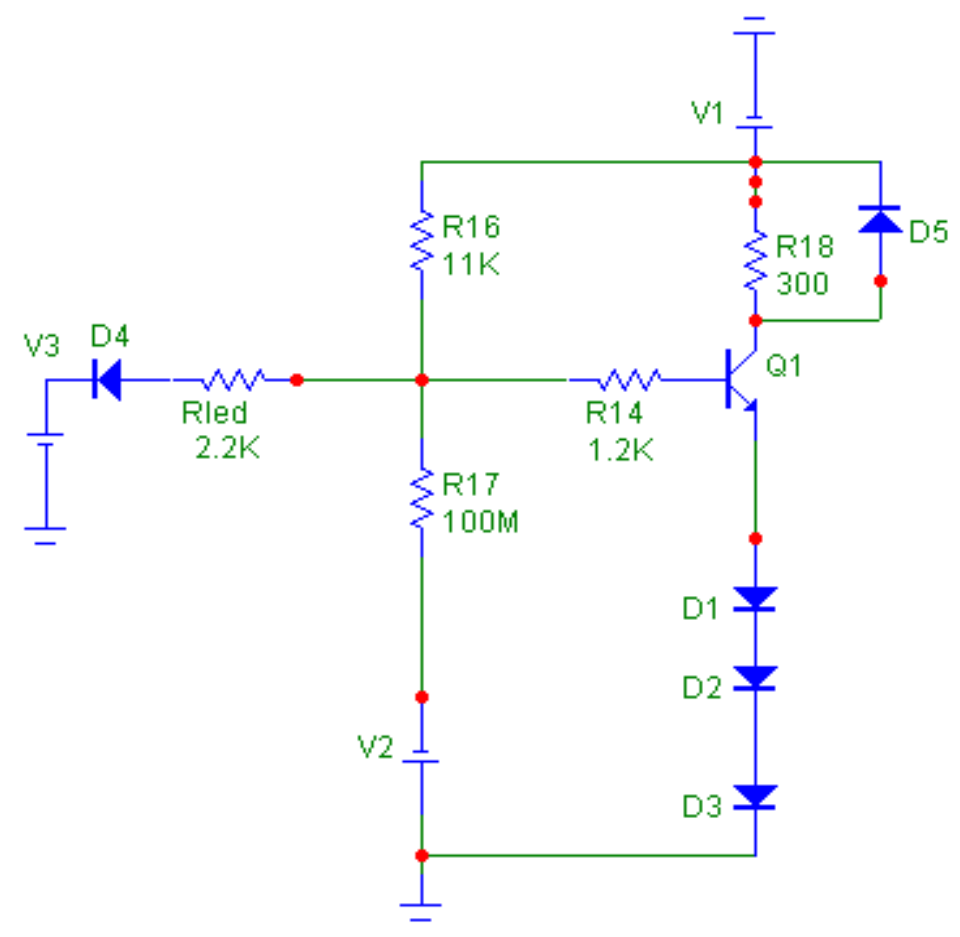

Figure A. 11: The operation of the Relay Driver.

\section{How to derive the Component Values}

According to the transistor datasheet $\mathrm{V}_{c e}$ (sat) is approximately $0.2 \mathrm{~V}$. The forward voltage across the diode is equal to $0.7 \mathrm{~V}$. Therefore, the current in the Relay becomes:

$$
\begin{aligned}
\mathrm{I}_{c} & =\frac{V_{\text {coil }}}{R_{\text {coil }}} \\
& =\frac{V_{c c}-V_{c e}-3\left(V_{d}\right)}{R_{c o i l}} \\
& =\frac{15 \mathrm{~V}-0.2 \mathrm{~V}-2.1 \mathrm{~V}}{300 \Omega} \\
& =42.3 \mathrm{~mA}
\end{aligned}
$$

The formula below is then used to determine the base current. Beta is chosen to 
be 50:

$$
\begin{aligned}
\mathrm{I}_{B} & =\frac{I_{c}}{\beta} \\
& =\frac{42.3 m A}{50} \\
& =0.846 \mathrm{~mA}
\end{aligned}
$$

Therefore we choose $I_{B}$ to be $1 \mathrm{~mA}$. Under normal operation the relay is switched and the LEDs are off.

$$
\begin{aligned}
\mathrm{I}_{B}=\frac{V_{c c}-V_{11}}{R_{16}+R_{14}} \\
\mathrm{R}_{16}+\mathrm{R}_{14}=\frac{15 \mathrm{~V}-2.8 \mathrm{~V}}{1 \mathrm{~mA}} \\
=12.2 \mathrm{k} \Omega
\end{aligned}
$$

$\mathrm{R}_{14}$ is chosen as $1.2 \mathrm{k} \Omega$, and therefore $\mathrm{R}_{16}$ becomes $11 \mathrm{k} \Omega . \mathrm{I}_{L E D}$ is chosen to be $2 \mathrm{~mA}$. When the base current becomes zero, the value of $\mathrm{R}_{L E D}$ becomes:

$$
\begin{aligned}
\mathrm{I}_{L E D} & =\frac{V_{c c}-V_{L E D}}{R_{L E D}+R_{16}} \\
& =\frac{15 \mathrm{~V}-1.8 \mathrm{~V}}{R_{L E D}+11 \mathrm{k} \Omega} \\
\mathrm{R}_{L E D} & =2.2 \mathrm{k} \Omega
\end{aligned}
$$

To get the value of $\mathrm{V}_{c}$ when the LED's are off, the following equation is applied:

$$
\begin{aligned}
\mathrm{V}_{c} & =\mathrm{V}_{c c}-\left(\mathrm{I}_{B} \times \mathrm{R}_{14}\right) \\
& =4 \mathrm{~V}
\end{aligned}
$$

When the base current is 0 and the transistor switches off, $\mathrm{V}_{c}$ becomes:

$$
\begin{aligned}
\mathrm{V}_{c} & =\frac{R_{L E D} \times V_{c c}}{R_{L E D}+R_{16}} \\
& =2.5 \mathrm{~V}
\end{aligned}
$$

\section{Simulation Program for the Relay Driver}

A WINDOW COMPARATOR

.OPTIONS LIST NODE POST 
Vin 50 SIN(0 125000 )

R7 12 10K

R8 $2340 \mathrm{~K}$

R9 34 10K

VS1 1015

vs2 0415

R10 71 10K

X12 51470 LM311

X25 31470 LM311

*LATCHING CIRCUIT

R11 $181.2 \mathrm{~K}$

R12 $841.8 \mathrm{~K}$

C3 $842 \mathrm{NF}$

*R13 $843.6 \mathrm{~K}$

X378 1470 LM311

* LM311 VOLTAGE COMPARATOR "MACROMODEL" SUBCIRCUIT

* CREATED USING PARTS VERSION 4.03 ON 03/07/90 AT 08:15

$* \mathrm{REV}(\mathrm{N} / \mathrm{A})$

* CONNECTIONS: NON-INVERTING INPUT

* I INVERTING INPUT

* || POSITIVE POWER SUPPLY

* $\quad$ || | NEGATIVE POWER SUPPLY

* $\quad$ || || OPEN COLLECTOR OUTPUT

* $\quad$ || ||| OUTPUT GROUND

* $\quad\|\| \|$ 
.SUBCKT LM311 123456

F1 93 V1 1

IEE 37 DC 100.0E-6

VI1 211 DC .45

VI2 222 DC .45

Q1 9217 QIN

Q2 8227 QIN

Q3 $984 \mathrm{QMO}$

Q4 $88 \quad 4$ QMI

.MODEL QIN PNP(IS=800.0E-18 BF=500)

.MODEL QMI NPN(IS=800.0E-18 BF=1002)

.MODEL QMO NPN(IS=800.0E-18 BF=1000 CJC=1E-15 TR=102.5E-9)

E1 11066961

V1 1011 DC 0

Q5 5116 QOC

.MODEL QOC NPN(IS=800.0E-18 $\quad$ BF=103.5E3 $\quad$ CJC=1E-15 TF=11.60E-12 $\mathrm{TR}=48.19 \mathrm{E}-9$ )

DP $43 \mathrm{DX}$

RP $3 \quad 46.667 \mathrm{E} 3$

.MODEL DX D(IS=800.0E-18)

ENDS

$*$

*A RELAY DRIVER

R1 110300

D5 101 D1N4148 
Q1 101112 Q2N2222A

D1 1213 D1N4148

D2 1314 D1N4148

D3 140 D1N4148

R14 $11151.2 \mathrm{~K}$

Rled $15162.2 \mathrm{~K}$

$\mathrm{R} 1615111 \mathrm{~K}$

R17 154 100meg

D4 167 D1N4148

.model D1N4148 D (IS=0.1PA, RS=16 CJO=2PF TT=12N BV=100 IBV=0.1PA)

*1N2222A NPN Transistor

. model Q2N2222A NPN $(\mathrm{IS}=14.34 \mathrm{~F}$ XTI=3 $\mathrm{EG}=1.11 \mathrm{VAF}=74.03 \quad \mathrm{BF}=255.9$

$+\mathrm{NE}=1.307 \mathrm{ISE}=14.34 \mathrm{~F} \quad \mathrm{IKF}=.2847 \mathrm{XTB}=1.5 \mathrm{BR}=6.092 \mathrm{NC}=2 \quad \mathrm{ISC}=0 \quad \mathrm{IKR}=0$

$+\mathrm{RC}=1 \mathrm{CJC}=7.306 \mathrm{P}$ MJC=.3416 $\mathrm{VJC}=.75 \mathrm{FC}=.5 \mathrm{CJE}=22.01 \mathrm{P} \mathrm{MJE}=.377$

$+\mathrm{VJE}=.75 \quad \mathrm{TR}=46.91 \mathrm{~N} \quad \mathrm{TF}=411.1 \mathrm{P} \quad \mathrm{ITF}=.6 \quad \mathrm{VTF}=1.7 \quad \mathrm{XTF}=3 \quad \mathrm{RB}=10)$

.TRAN $.5 \mathrm{~m} 25 \mathrm{~m}$

.PLOT TRAN V(2) V(5) V(3) v(7) V(8) V(15) V(12) V(10) V(7) V(11,12) V(11) END 


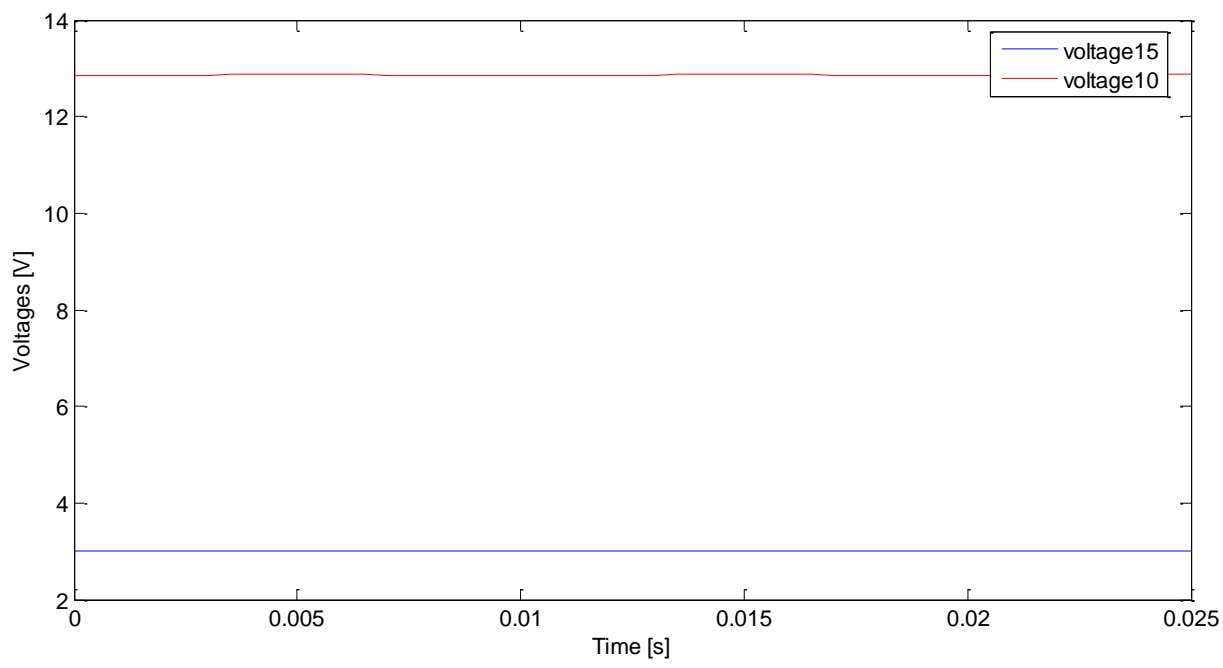

Figure A. 12: The Relay Driver before the reset button is pressed.

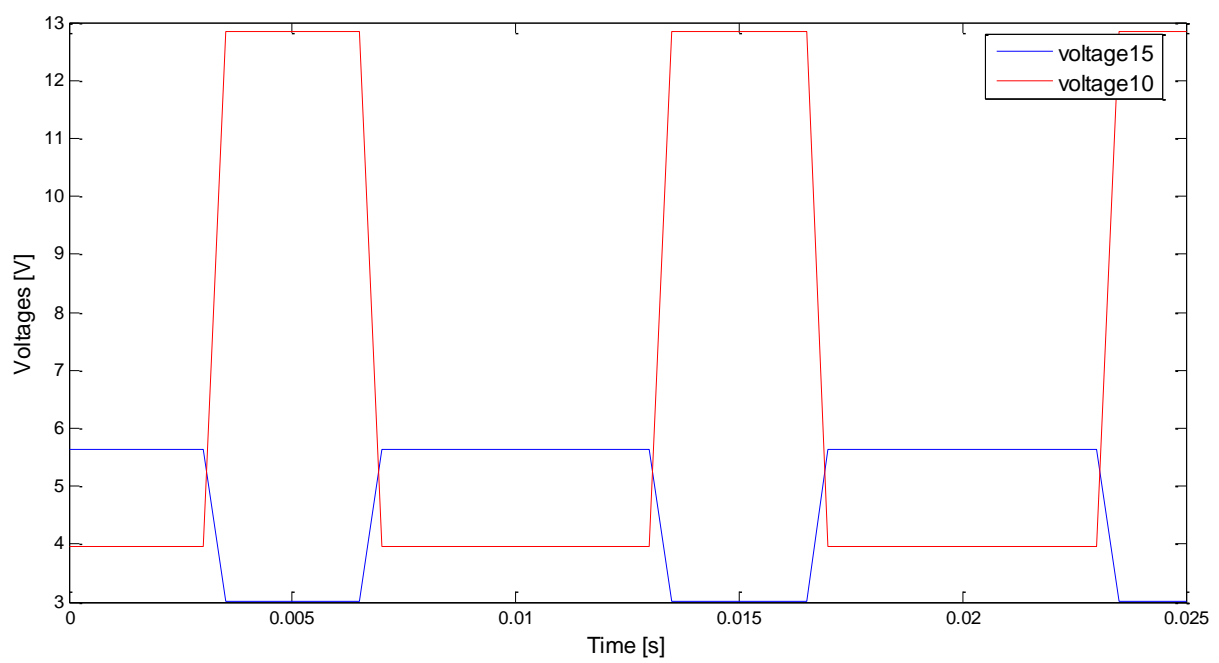

Figure A. 13: The Relay Driver when the reset button is pressed. 


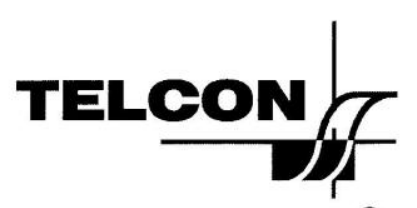

Speciality Magnetic Components

QUALIFIED TO ISO 9002

\section{PCB Mounting Hall Effect Current Transformer Type HTP25}

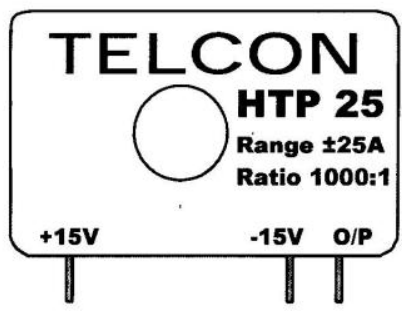

The HTP25 is a closed loop Hall Effect Current Transformer suitable for measuring currents up to 25A. The product provides an output current into an external load resistance.

\section{Features}

- High Accuracy

- 3kV Proof Stress

- Fast Response

- Designed In Quality

Applications

- Variable Speed Drives

- UPS Systems

- D.C Power Supplies

- Low Frequency Current Measurement

\section{Benefits}

- Galvanic Isolation

- Wide Dynamic Range

- Non - Invasive

- High Reliability.

- Overcurrent Protection

- Robotics

- Frequency Inverters

- Power Factor Monitoring

\begin{tabular}{ll} 
TELCON LIMITED & Manor Royal \\
Telephone: $\quad+441293528800$ & Crawley \\
Fax: $\quad+441293524466$ & West Sussex RH10 2PY \\
e-mail: general@telcon.co.uk & England \\
www.telcon.co.uk & \\
\hline SALES Phone: +441293558800 & Fax: +44 1293 523883 \\
\hline
\end{tabular}

Registered Office: Hill House, Little New Street, LONDON EC4A 3TR, England No 1741741 


\section{TECHNICAL DATA}

Nominal Primary Current

Turns Ratio

25A

Nominal Power Supply

Supply Current

Minimum Load Resistance

Operating Temperature Range

Storage Temperature Range

1000:1

$\pm 15 \mathrm{~V} \pm 5 \%$

$16 \mathrm{~mA}$ per rail + output current

$150 \Omega$

0 to $+70^{\circ} \mathrm{C}$

$-25^{\circ} \mathrm{C}$ to $+85^{\circ} \mathrm{C}$

\section{SPECIFICATION}

Linearity

Limit of linearity

Overall accuracy

Output Offset Current

Zero Offset /Temperature

Zero Offset/Supply Variation

Coil Resistance

Bandwidth (-1dB)

di/dt following

Delay time

dV/dt Immunity

Proof Stress Voltage
$0.1 \%$ of nominal primary current

$\pm 55 \mathrm{~A}$ peak

$0.5 \%$ of nominal primary current

$< \pm 200 \mu \mathrm{A}$ at primary current $=0 \mathrm{~A}$

$<5 \mu \mathrm{A} /{ }^{\circ} \mathrm{C}$

$<5 \mu \mathrm{A} N$

$52 \Omega$

dc to $200 \mathrm{kHz}$

$>200 \mathrm{~A} / \mu \mathrm{s}$

$0.1 \mu \mathrm{S}$

$10 \mathrm{kV} / \mu \mathrm{s}$

$3 \mathrm{kV}$ a.c., r.m.s, $50 \mathrm{~Hz}$ for 1 minute

\section{GENERAL DATA}

Weight

Housing

Mounting

Signal Sense

Conductor Temperature

Conductor Position
$17 \mathrm{~g}$ nominal

Modified Polyphenylene Oxide

Direct mounting to PCB by 3 pins

Positive output obtained when current flows in the direction of the arrow

The temperature of the primary conductor should not exceed $100^{\circ} \mathrm{C}$

Optimum dynamic performance is achieved with a single conductor filling the bore

\section{DIMENSIONS}
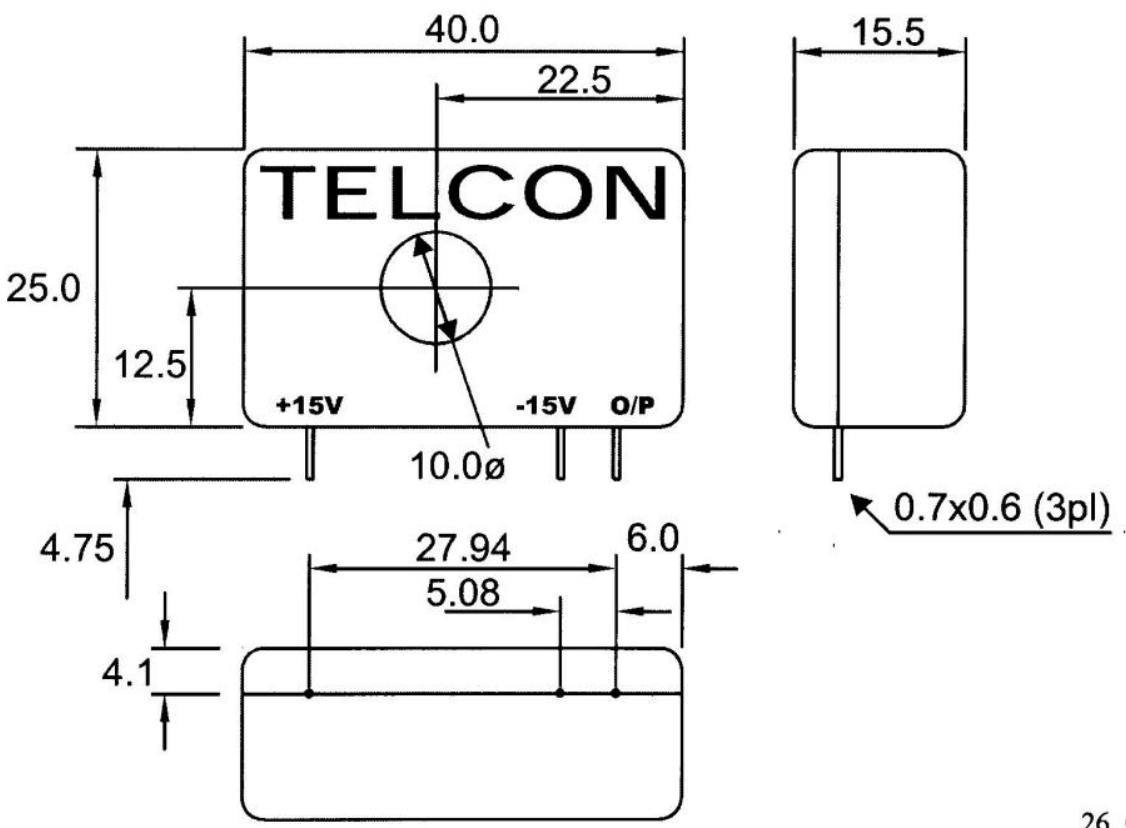


\section{Appendix B}

\section{Electric Field Simulation of a Silicone Rubber Insulator}

The electric field distributions for three Silicone Rubber (SR) insulator rods were simulated using the Electro finite-boundary element simulation software program as part of the preliminary investigation for the project. Three creepage distances are used, namely $277 \mathrm{~mm}, 346 \mathrm{~mm}$ and $433 \mathrm{~mm}$. The conducting end-pieces on the live side and earth side are $30 \mathrm{~mm}$ and $34 \mathrm{~mm}$ respectively. A $10 \mathrm{kV}$ r.m.s voltage is applied on the live side of the insulator rod.

There following conditions were investigated:

- The maximum electric field strength for a clean Insulator.

- $\quad$ The maximum electric field strength for a polluted and conductive insulator.

- $\quad$ The maximum electric field strength for an insulator with dry band activity.

There electro simulation for a clean insulator in Fig B.1 shows that the maximum electric field is concentrated at the sharp ends (signified by the red color). Furthermore, the closer the contours are, the higher the electric field. There maximum electric field is given as $0.4204 \mathrm{kV} / \mathrm{mm}$.
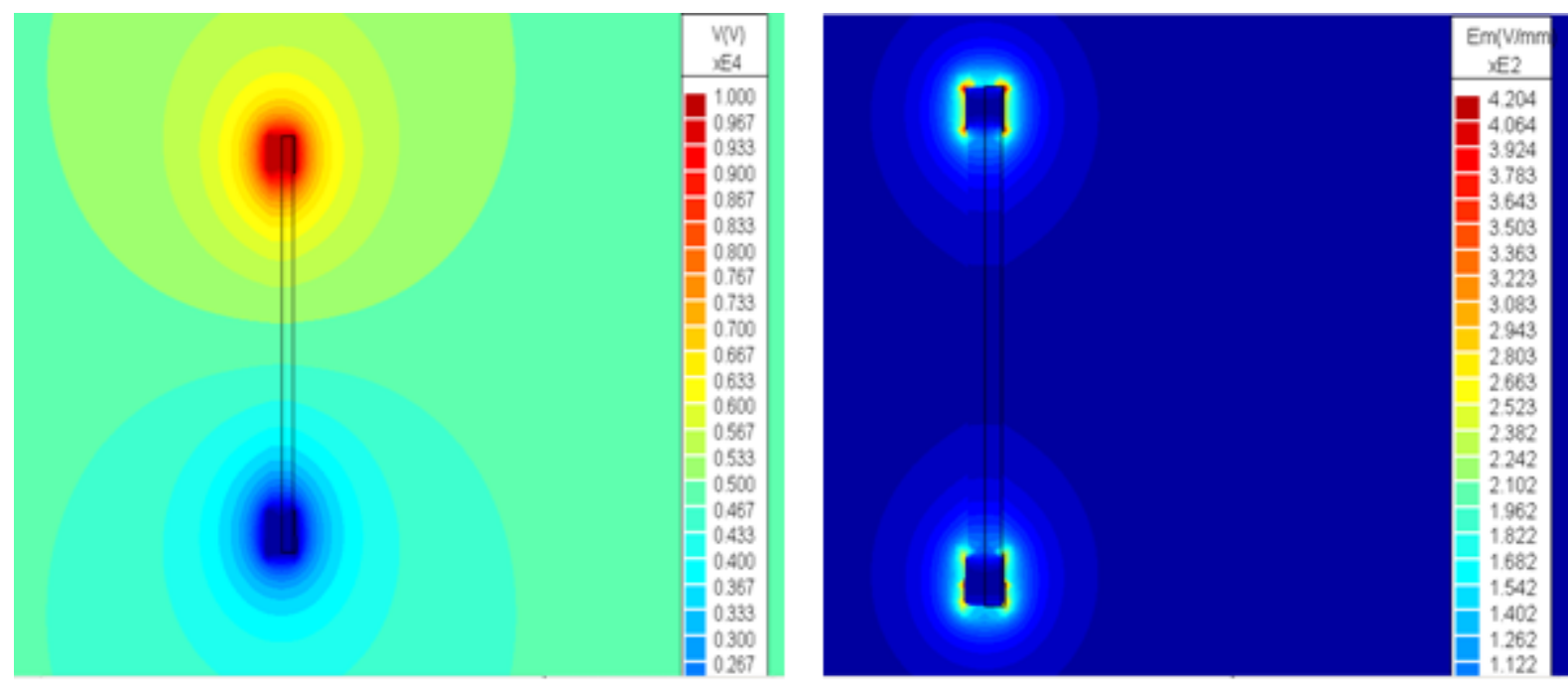

Figure B. 1: Potential and field strength contours for a clean insulator with creepage distance of $28 \mathrm{~mm} / \mathrm{kV}$

However, when the insulator gets polluted with a conductive layer (shown in Fig B.2), the electric field becomes more uniform more evenly spread. Even though, the maximum electric field is still at the sharp ends (signified by the red color). There 
max electric field is given as $0.3282[\mathrm{kV} / \mathrm{mm}]$. In other words, there is a decrease in the maximum electric field value when the insulator gets polluted.
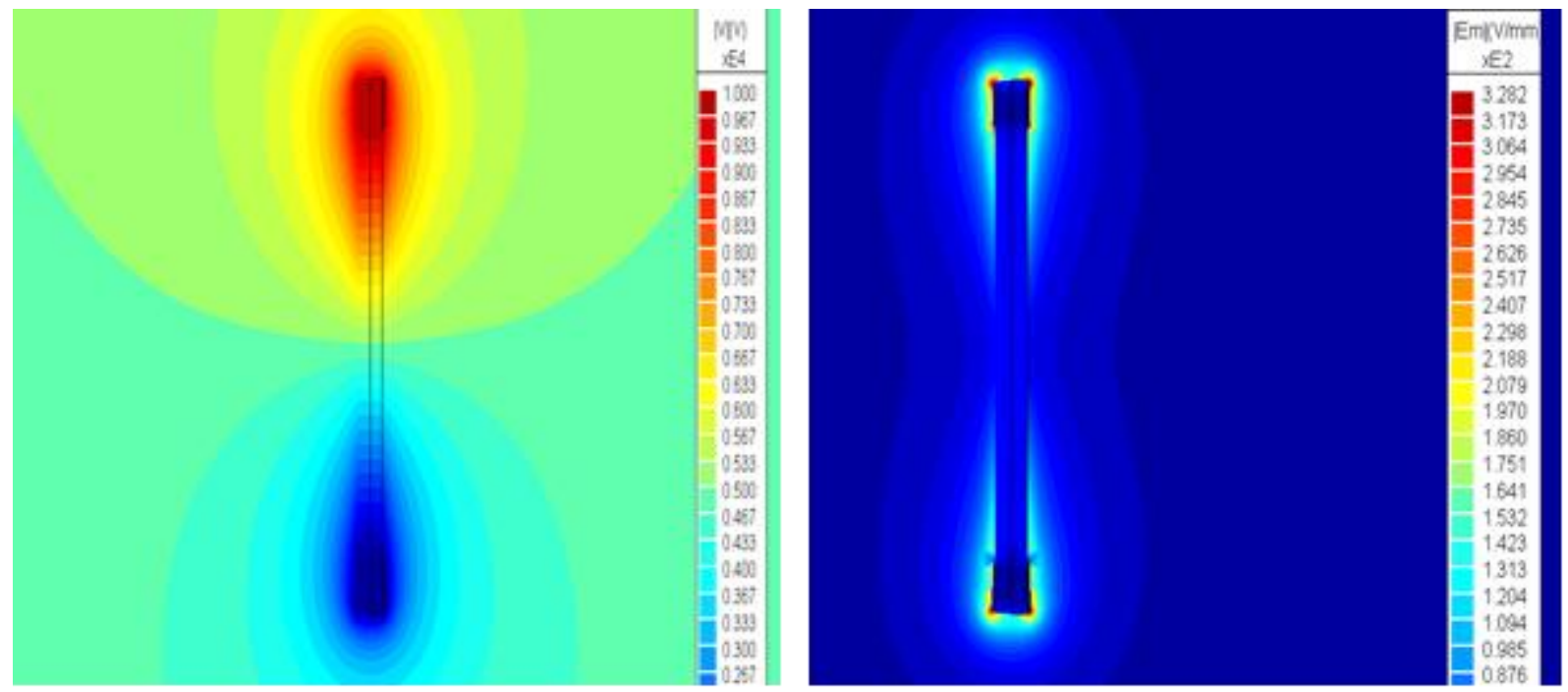

Figure B. 2: Potential and field strength contours for a polluted insulator with creepage distance of $28 \mathrm{~mm} / \mathrm{kV}$

However, when the insulator becomes conductive and has dry band activity, the electric field is concentrated in the region of the dry band (shown in Fig B.3). There maximum electric field is given as $0.6875 \mathrm{kv} / \mathrm{mm}$. The value of the maximum electric field increases. These simulations were done for different $\mathrm{S}_{C D}$, but no correlation was found. There simulation for the other creepage distances is given in Appendix B, but it is also summarized in Table B.1.
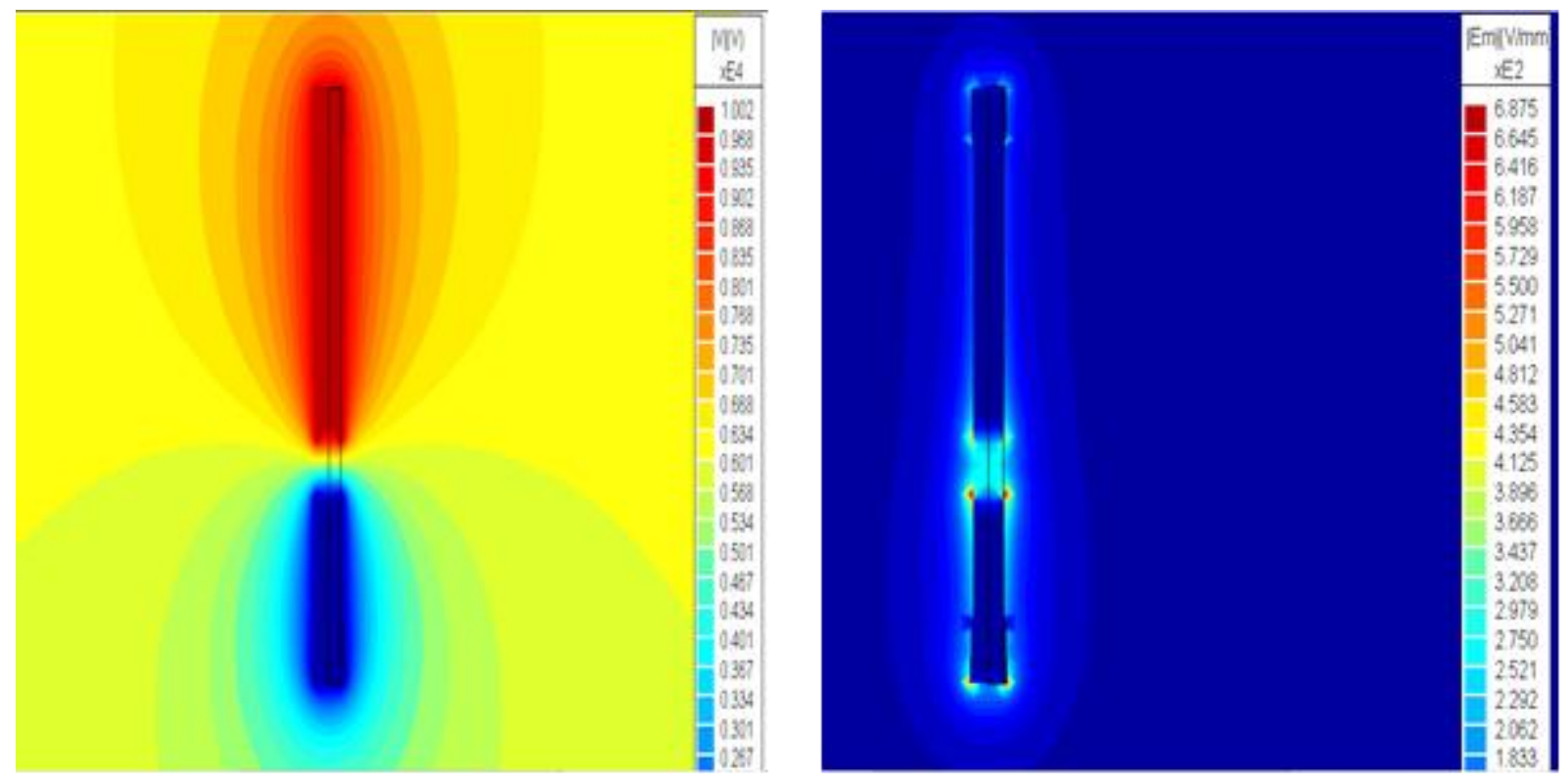

Figure B. 3: Potential and field strength contours for a conductive insulator with a dryband with creepage distance of $28 \mathrm{~mm} / \mathrm{kV}$ 
The electro simulation for the insulator with different $S_{C D}$ is given below, starting with the $\mathrm{S}_{C D}$ of $35[\mathrm{~mm} / \mathrm{kV}]$ below.
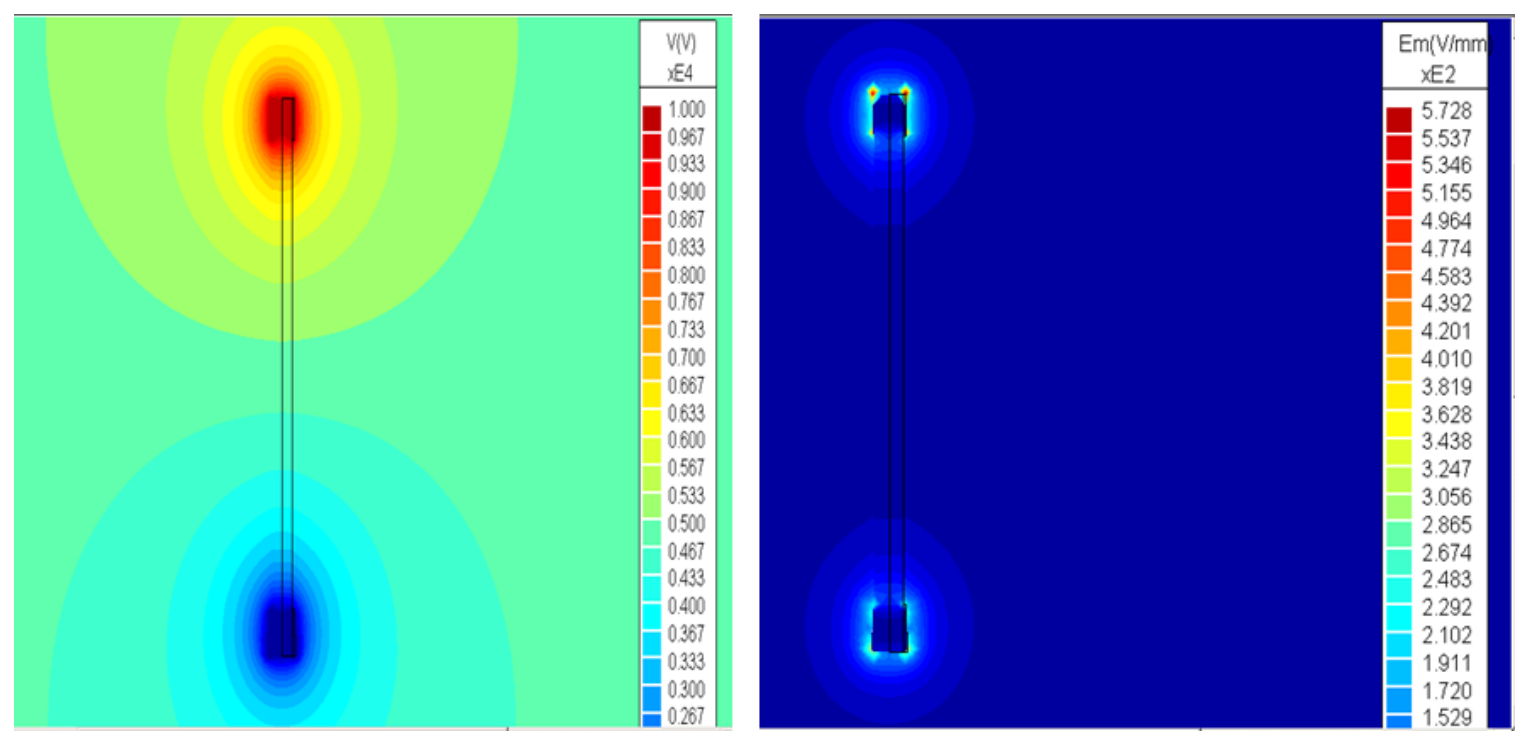

Figure B. 4: Potential and field strength contours for a clean insulator with creepage distance of $35 \mathrm{~mm} / \mathrm{kV}$
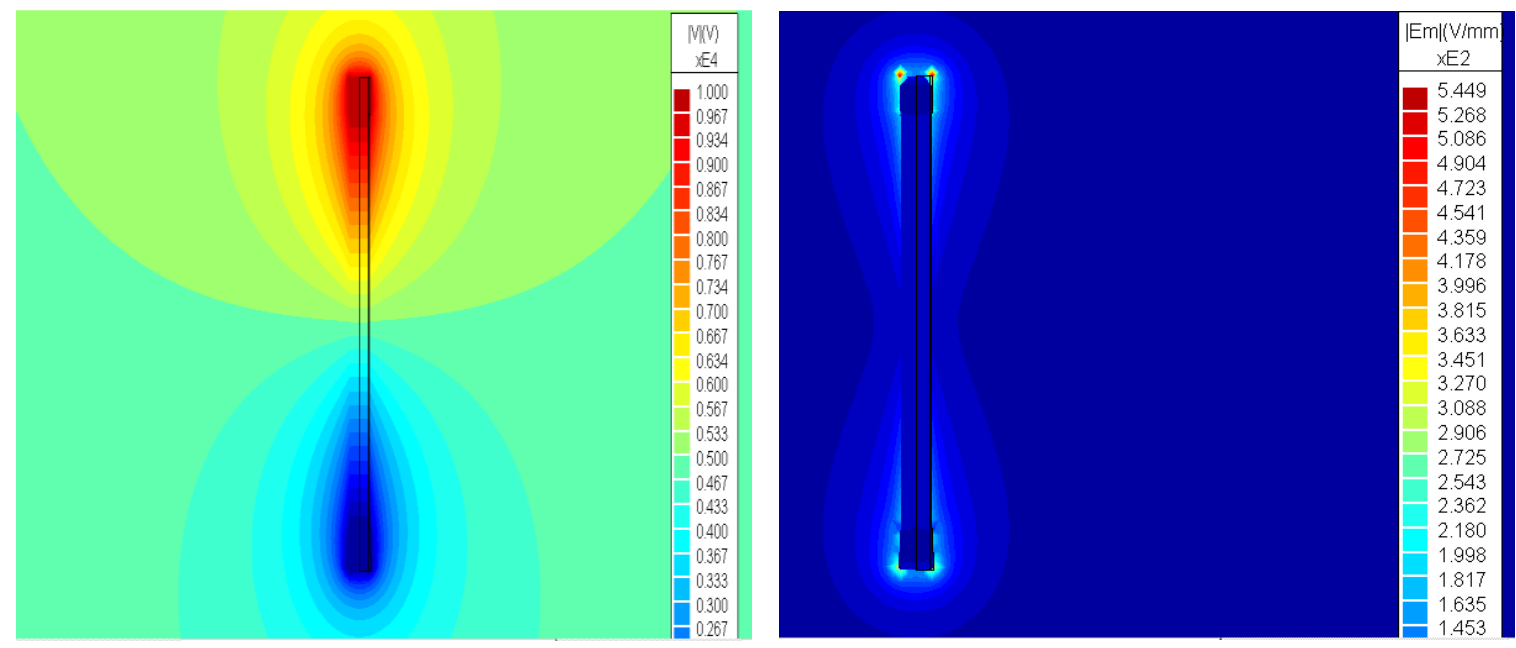

Figure B. 5: Potential and field strength contours for a polluted insulator with creepage distance of $35 \mathrm{~mm} / \mathrm{kV}$ 

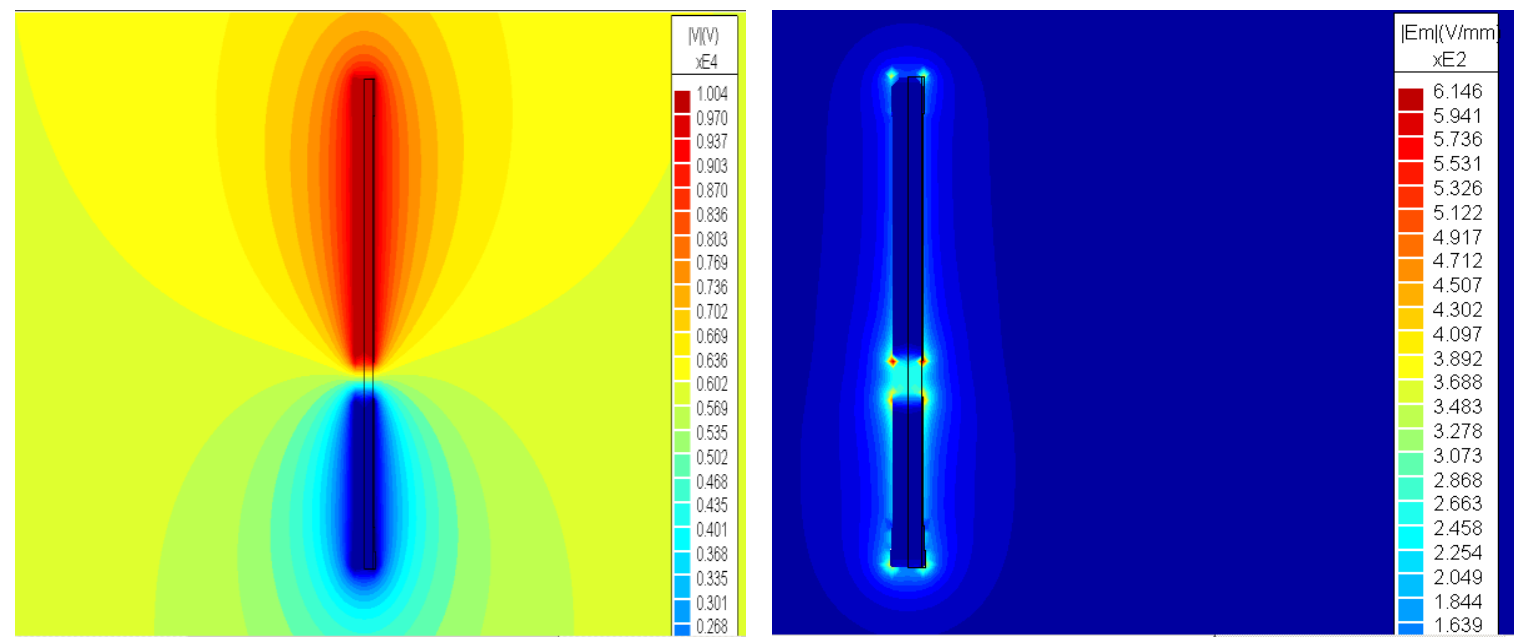

Figure B. 6: Potential and field strength contours for a conductive insulator with a dryband with creepage distance of $35 \mathrm{~mm} / \mathrm{kV}$ ].
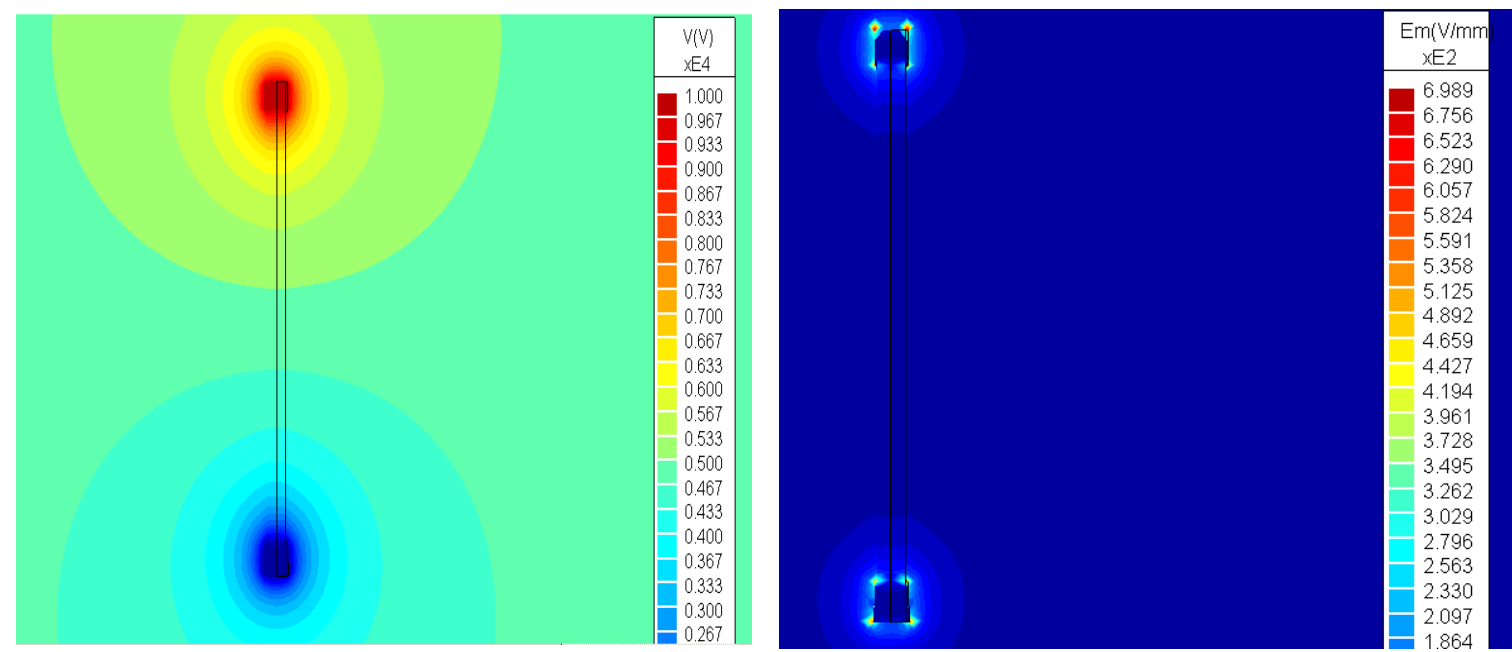

Figure B. 7: Potential and field strength contours for a clean insulator with creepage distance of $43 \mathrm{~mm} / \mathrm{kV}$ 

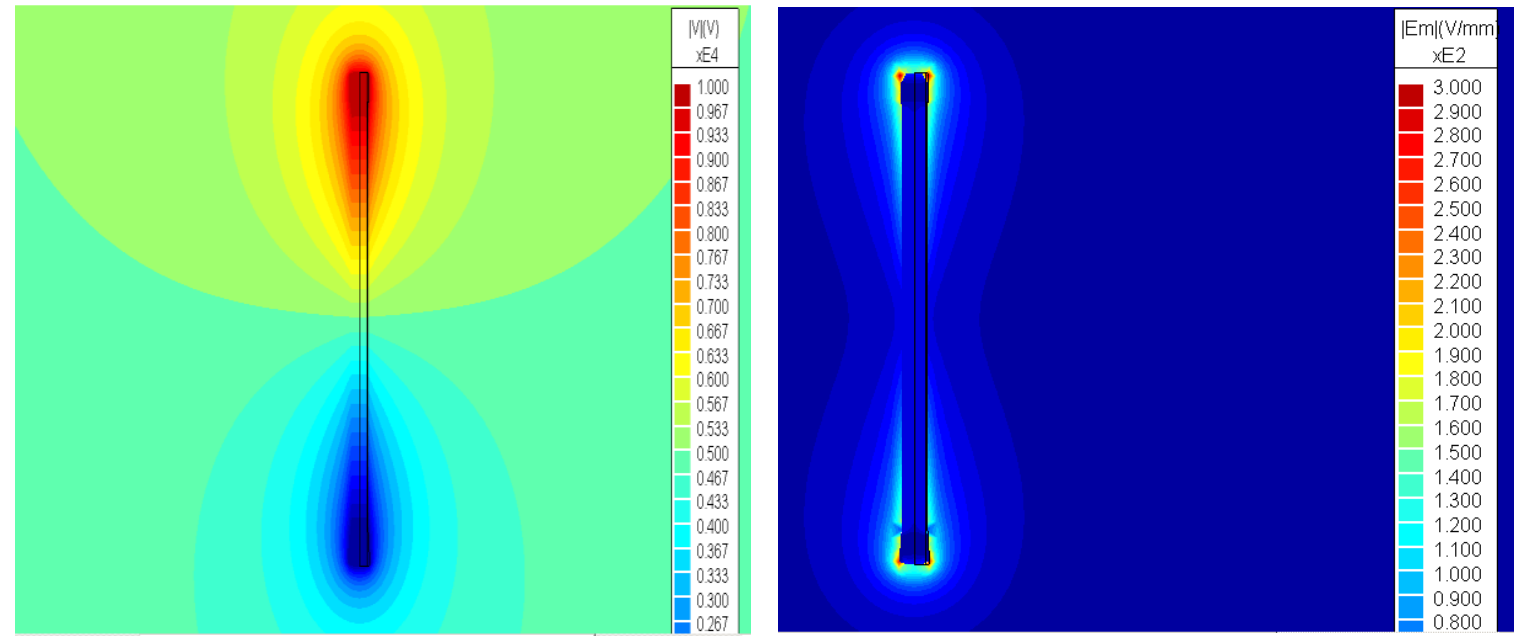

Figure B. 8: Potential and field strength contours for a polluted insulator with creepage distance of $43 \mathrm{~mm} / \mathrm{kV}$
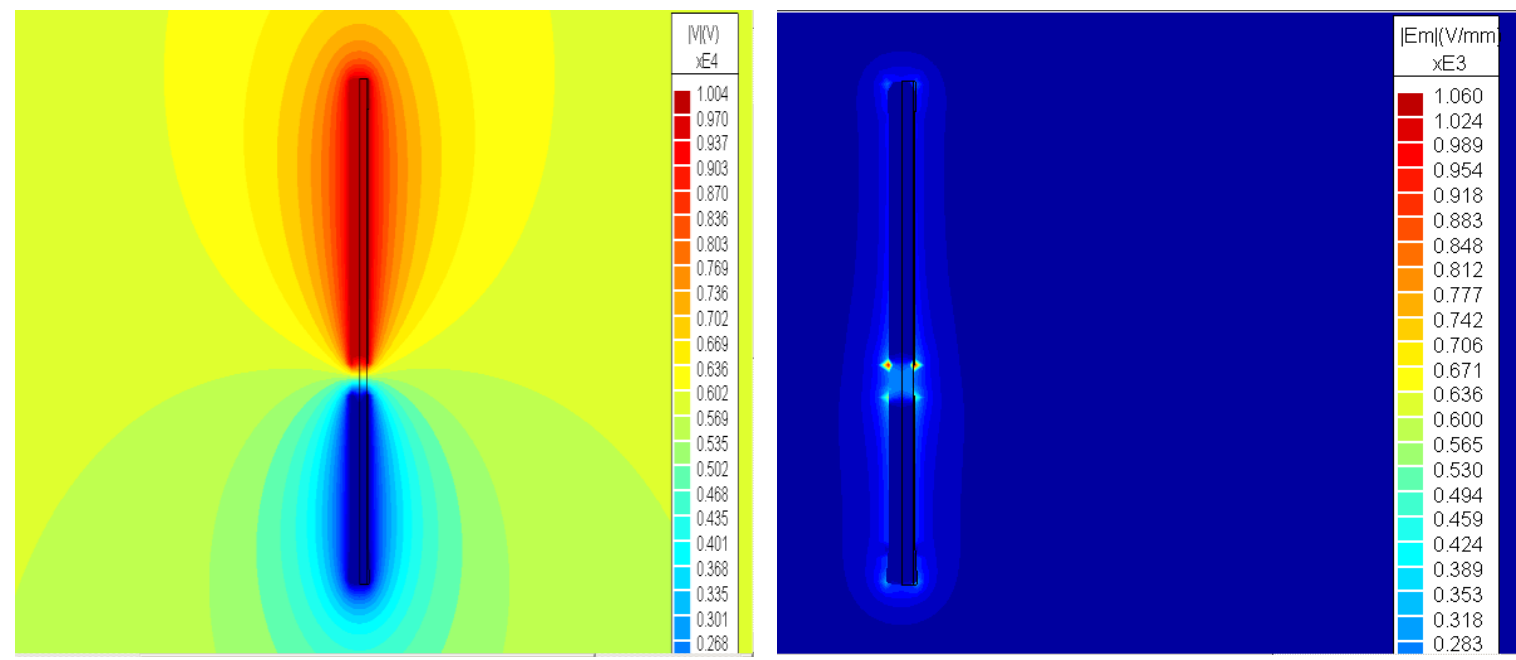

Figure B. 9: Potential and field strength contours for a conductive insulator with a dryband with creepage distance of $43 \mathrm{~mm} / \mathrm{kV}$

Table B.1 summarizes the results for the different creepage. 
Table B.6.1: Table for Electro Field Simulation

\begin{tabular}{|r|l|l|l|}
\hline $\begin{array}{l}\mathbf{S}_{C D} \\
{[\mathrm{~mm} / \mathrm{kV}]}\end{array}$ & $\begin{array}{l}\text { Creepage distance } \\
{[\mathrm{mm}]}\end{array}$ & Status of the insulator & $\begin{array}{l}\text { Maximum Electric Field } \\
{[\mathrm{kV} / \mathrm{mm}]}\end{array}$ \\
\hline 28 & 277 & Clean & 0.4204 \\
\hline & & Polluted and conductive & 0.3282 \\
\cline { 3 - 5 } & & Dry band and conductive & 0.6875 \\
\hline 35 & 346 & Clean & 0.5728 \\
\hline & & Polluted and conductive & 0.5449 \\
\cline { 3 - 5 } & & Dry band and conductive & 0.6146 \\
\hline 43 & & Clean & 0.6989 \\
\hline & & Polluted and conductive & 0.3 \\
\cline { 3 - 4 } & & Dry band and conductive & 1.06 \\
\hline
\end{tabular}




\section{Appendix C}

\section{The Tracking Wheel Test Standards}

\section{Annex A}

(normative)

\section{Wheel test}

\section{A.1 Test specimens}

Two test insulators of identical design with a creepage distance between $500 \mathrm{~mm}$ and $800 \mathrm{~mm}$ shall be taken from the production line. If such insulators cannot be taken from the production line, special test specimens shall be made from other insulators so that the creepage distance falls between the given values. These special test specimens shall be fitted with standard production end fittings.

Up to two pairs of test specimens can be tested simultaneously on one wheel. It is recommended not to mix widely differing materials on the same wheel.

\section{A.2 Procedure}

The test specimens shall be cleaned with de-ionized water before starting the test. The test specimens are mounted on the wheel as shown in Figure A.1 below. They go through four positions in one cycle. Each test specimen remains stationary for about $40 \mathrm{~s}$ in each of the four positions. The $90^{\circ}$ rotation from one position to the next takes about $8 \mathrm{~s}$. In the first part of the cycle the insulator is dipped into a saline solution. The second part of the test cycle permits the excess saline solution to drip off the specimen ensuring that the light wetting of the surface gives rise to sparking across dry bands that will form during the third part of the cycle. In that part the specimen is submitted to a power frequency voltage. In the last part of the cycle the surface of the specimen that had been heated by the dry band sparking is allowed to cool.

The test voltage is supplied by a test transformer. When loaded with a resistive current of $250 \mathrm{~mA}$ on the high voltage side the test circuit shall exhibit a maximum drop of $5 \%$ in its output voltage.

The salt solution shall be replaced weekly. Weekly interruptions of the test for inspection purposes, each of these not exceeding $1 \mathrm{~h}$ are permissible. Interruption periods will not be counted in the test duration. One longer interruption up to $60 \mathrm{~h}$ is allowed. An additional testing time of three times the duration of the interruption period shall be added. The final test report shall include details of all interruptions.

\section{Á.3 Test conditions}

Electrical stress:

$\mathrm{NaCl}$ content of de-ionized water: Ambient temperature:

Test duration:
The power frequency test voltage in $\mathrm{kV}$ is determined by dividing the actual creepage distance in millimetres by 28,6 .

$1,40 \mathrm{~kg} / \mathrm{m}^{3} \pm 0,06 \mathrm{~kg} / \mathrm{m}^{3}$

$20{ }^{\circ} \mathrm{C} \pm 5 \mathrm{~K}$

30000 cycles

\section{A.4 Acceptance criteria}

The test specimens of identical design shall be assessed together. Pairs of test specimens of different design shall be assessed separately. The numbers of flashovers and trip-outs shall be recorded and noted in the test report. 
The test is regarded as passed, if on both test specimens:

- no tracking occurs, (a megohmmeter shall be applied along any suspect path, using $1 \mathrm{kV}$ DC or higher. The probes shall be between $5 \mathrm{~mm}$ to $10 \mathrm{~mm}$ apart. A resistance of less than $2 \mathrm{M} \Omega$ shall constitute failure);

- for composite insulators: erosion depth is less than $3 \mathrm{~mm}$ and does not reach the core, if applicable;

- for resin insulators: erosion depth is less than $3 \mathrm{~mm}$;

- no shed, housing or interface is punctured.

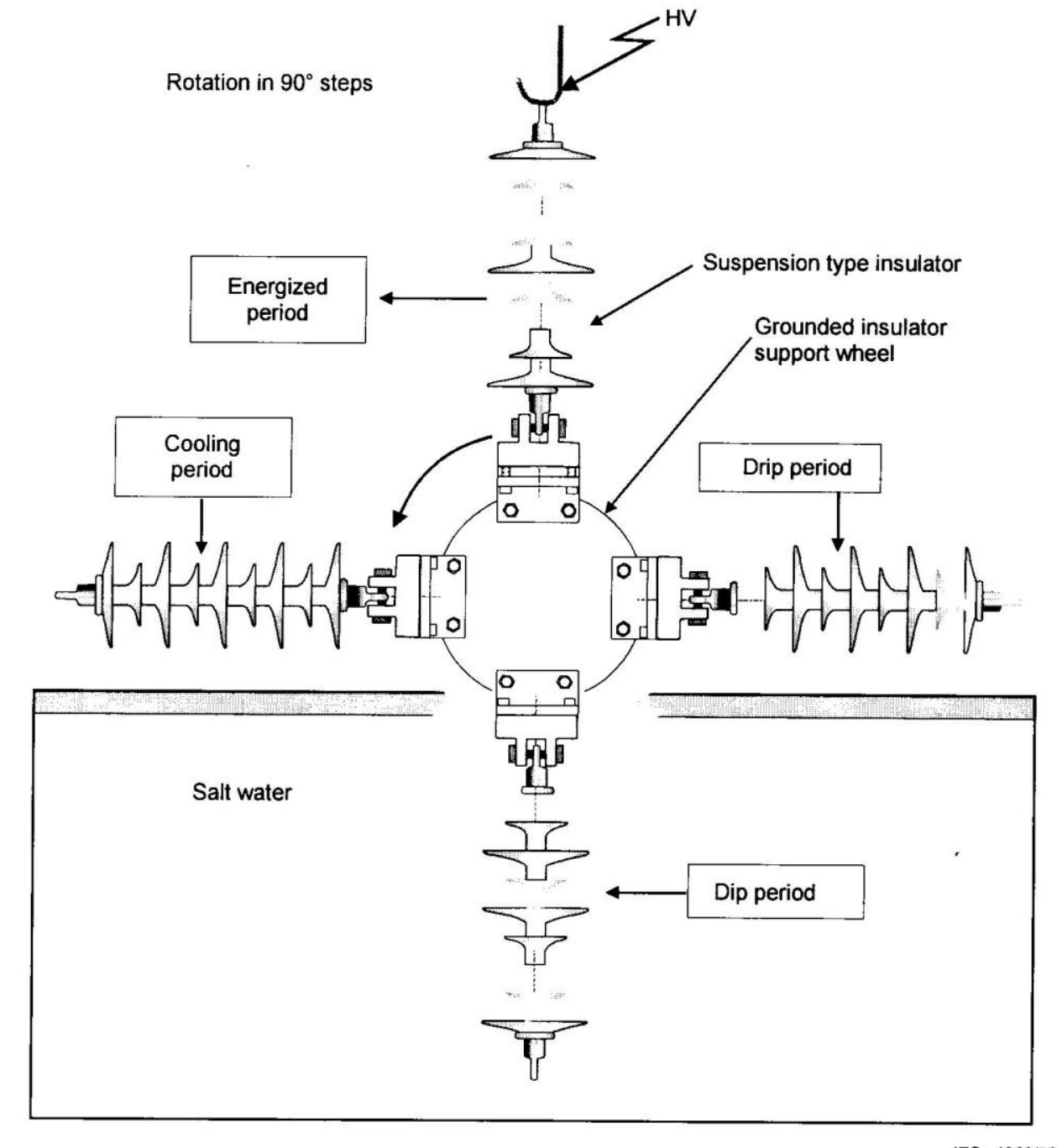

IEC 1960/05

Figure A.1 - Test arrangement of the tracking wheel test 


\section{Appendix D}

\section{Design of the DC Source}

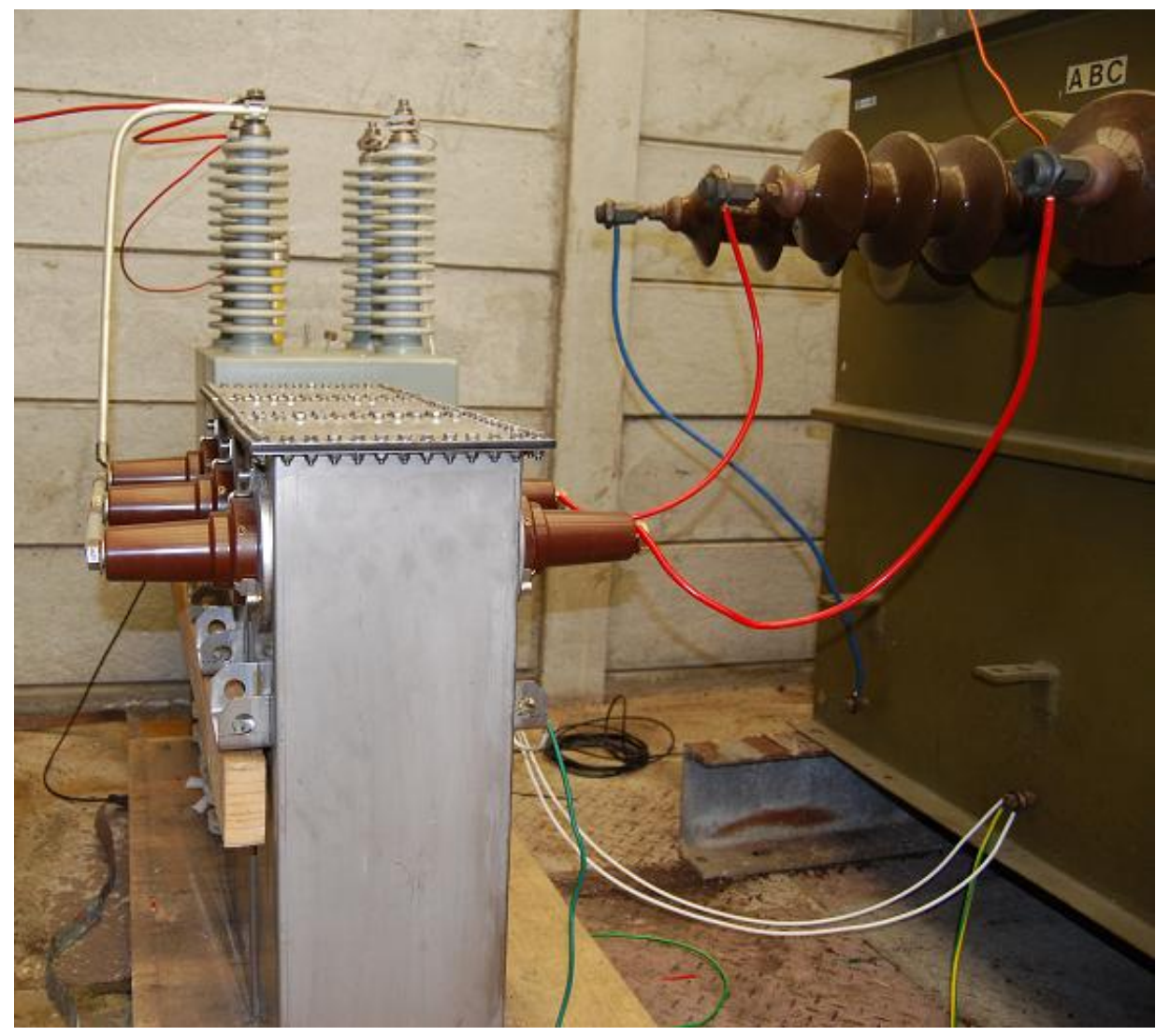

Figure D. 1: The DC Source used for 2nd, 3rd and 4th TWT experiment

Table D. 1: Specification for the Capacitor Bank

\begin{tabular}{|l|l|l|l|}
\hline Serial Number & Style Number & Type & Impregnant \\
$071 Z 001$ & GMA682R & FILMVAR & NON PCB \\
\hline Rated Voltage & Rated Output & Rated Cap. & Actual Cap. \\
$12500 \mathrm{~V}$ & $429.51 \mathrm{kVAR}$ & $8.75 \mathrm{mfd}$ & $8.54 \mathrm{mfd}$ \\
\hline Phase & INT Connection & Connection & Insulation \\
1 & $\mathrm{~S}=7 \mathrm{P}=4$ & 1 & $50 / 200 \mathrm{kV}$ \\
\hline Frequency & Discharge Res. & Fluid Volume & Unit Mass \\
$50 \mathrm{~Hz}$ & $4.6 \mathrm{MOHM}$ & $15 \mathrm{~L}$ & $64 \mathrm{~kg}$ \\
\hline Fuse & Temp Cat & Specification & Date \\
EXT & $-25 /+50 \mathrm{C}$ & IEC $60871-1$ & $19 / 2 / 08$ \\
\hline
\end{tabular}


Table D. 2: Transformer Specification (cbayn configuration)

\begin{tabular}{|l|l|}
\hline Rated Power & $100 \mathrm{kVA}$ \\
\hline Rated Voltages & $22000 / 400 \mathrm{~V}$ \\
\hline Rated Currents & $2.62 / 144.3 \mathrm{~A}$ \\
\hline Impedance Voltages & $4.29 \%$ \\
\hline Core \& Windings & $340 \mathrm{~kg}$ \\
\hline Oil & $228 \mathrm{~kg}$ \\
\hline Total Mass & $732 \mathrm{~kg}$ \\
\hline Conductor Material & $\mathrm{Al} / \mathrm{Al}$ \\
\hline
\end{tabular}

Table D. 3: Conductivity Table for the 1st HVAC experiment

\begin{tabular}{|c|c|c|c|c|c|c|}
\hline \begin{tabular}{|l} 
Solution Temperature \\
{$\left[\begin{array}{ll}{ }^{\circ} \mathrm{C} & ]\end{array}\right.$}
\end{tabular} & $\mathrm{Ts}^{\wedge} \mathbf{2}$ & $\mathrm{Ts}^{\wedge} 3$ & $\mathrm{Kt}$ & $\left.\begin{array}{c}\sigma \\
\sigma[\mathrm{mS} / \mathrm{cm}]\end{array}\right]$ & $\begin{array}{c}\sigma_{20} \\
{[\mathrm{~ms} / \mathrm{cm}]}\end{array}$ & \\
\hline 10 & 100 & 1000 & 0.028168 & 0.930247 & & \\
\hline 11 & 121 & 1331 & 0.027547 & 0.955411 & & \\
\hline 12 & 144 & 1728 & 0.026944 & 0.980851 & & \\
\hline 13 & 169 & 2197 & 0.02636 & 1.006549 & & \\
\hline$\overline{14}$ & 196 & 2744 & 0.025794 & 1.032486 & & \\
\hline 15 & 225 & 3375 & 0.025246 & 1.058646 & & \\
\hline 16 & 256 & 4096 & 0.024716 & \begin{tabular}{|l|}
1.085011 \\
\end{tabular} & & \\
\hline 17 & 289 & 4913 & 0.024203 & 1.111569 & & \\
\hline 18 & 324 & 5832 & 0.023707 & 1.138306 & & \\
\hline 19 & 361 & 6859 & 0.023229 & 1.165212 & & \\
\hline 20 & 400 & 8000 & 0.022768 & \begin{tabular}{|l|}
1.192279 \\
\end{tabular} & & \\
\hline 21 & 441 & 9261 & 0.022324 & 1.219502 & & \\
\hline 22 & 484 & 10648 & 0.021896 & 1.246881 & & \\
\hline 23 & 529 & \begin{tabular}{c|c|}
12167 \\
\end{tabular} & 0.021484 & 1.274419 & & \\
\hline 24 & 576 & 13824 & 0.021089 & 1.302121 & & \\
\hline 25 & 625 & 15625 & 0.02071 & 1.33 & & 1.1922785 \\
\hline 26 & 676 & 17576 & 0.020347 & 1.358072 & & \\
\hline 27 & 729 & 19683 & 0.019999 & 1.386359 & & \\
\hline 28 & 784 & 21952 & 0.019667 & 1.414889 & & \\
\hline 29 & 841 & 24389 & 0.01935 & \begin{tabular}{|l|}
1.443697 \\
\end{tabular} & & \\
\hline 30 & 900 & 27000 & 0.019048 & 1.472822 & & \\
\hline
\end{tabular}


Table D. 4: Conductivity Table for the 2nd, 3rd and 4th experiment

\begin{tabular}{|c|c|c|c|c|c|}
\hline $\begin{array}{l}\text { Solution Temperature } \\
{\left[\begin{array}{ll}{ }^{\circ} C & ]\end{array}\right.}\end{array}$ & $T s^{\wedge} 2$ & $T s^{\wedge} 3$ & Kt & $\begin{array}{ll}\sigma & t \\
{[\mathrm{mS} / \mathrm{cm}]}\end{array}$ & $\left.\begin{array}{c}\sigma_{20} \\
{[\mathrm{~ms} / \mathrm{cm}]}\end{array}\right]$ \\
\hline 10 & 100 & 1000 & 0.028168 & 1.897656 & \\
\hline 11 & 121 & 1331 & 0.027547 & 1.948989 & \\
\hline 12 & 144 & 1728 & 0.026944 & 2.000886 & \\
\hline 13 & 169 & 2197 & 0.02636 & 2.053308 & \\
\hline 14 & 196 & 2744 & 0.025794 & 2.106219 & \\
\hline 15 & 225 & 3375 & 0.025246 & 2.159583 & \\
\hline 16 & 256 & 4096 & 0.024716 & 2.213368 & \\
\hline 17 & 289 & 4913 & 0.024203 & 2.267544 & \\
\hline 18 & 324 & 5832 & 0.023707 & 2.322086 & \\
\hline 19 & 361 & 6859 & 0.023229 & 2.376972 & \\
\hline 20 & 400 & 8000 & 0.022768 & 2.432187 & \\
\hline 21 & 441 & 9261 & 0.022324 & 2.487722 & \\
\hline 22 & 484 & 10648 & 0.021896 & 2.543574 & \\
\hline 23 & 529 & 12167 & 0.021484 & 2.599749 & \\
\hline 24 & 576 & 13824 & 0.021089 & 2.65626 & \\
\hline 25 & 625 & 15625 & 0.02071 & 2.713132 & 2.43218734 \\
\hline 26 & 676 & 17576 & 0.020347 & 2.770398 & \\
\hline 27 & 729 & 19683 & 0.019999 & 2.828102 & \\
\hline 28 & 784 & 21952 & 0.019667 & 2.886302 & \\
\hline 29 & 841 & 24389 & 0.01935 & 2.945067 & \\
\hline 30 & 900 & 27000 & 0.019048 & 3.004481 & \\
\hline 31 & 961 & 29791 & 0.018761 & 3.064641 & \\
\hline 32 & 1024 & 32768 & 0.018489 & 3.12566 & \\
\hline 33 & 1089 & 35937 & 0.018231 & 3.187671 & \\
\hline 34 & 1156 & 39304 & 0.017987 & 3.25082 & \\
\hline 35 & 1225 & 42875 & 0.017758 & 3.315278 & \\
\hline
\end{tabular}

Table D. 5: The Salinity $\left[\mathrm{kg} / \mathrm{m}^{\wedge} 3\right]$ vs Volume Conductivity $\sigma_{20}[\mathbf{S} / \mathbf{m}]$

\begin{tabular}{|r|r|l|}
\hline $\begin{array}{l}\text { Salinity (Sa) } \\
{\left[\mathrm{kg} / \mathrm{m}^{\wedge} 3\right]}\end{array}$ & $\begin{array}{l}\text { Volume Conductivity } \\
\sigma_{20}[\mathrm{~S} / \mathrm{m}]\end{array}$ & $\begin{array}{l}\text { Pollution Severity } \\
\text { Class }\end{array}$ \\
\hline 2.5 & 0.43 & Light \\
\hline 3.5 & 0.6 & Light \\
\hline 5 & 0.83 & Light \\
\hline 7 & 1.15 & Light \\
\hline 10 & 1.6 & Light \\
\hline 14 & 2.2 & Light \\
\hline 20 & 3 & Medium \\
\hline 28 & 4.1 & Medium \\
\hline 40 & 5.6 & Medium \\
\hline 56 & 7.6 & Heavy \\
\hline 80 & 10 & Heavy \\
\hline 112 & 13 & Heavy \\
\hline 160 & 17 & Very heavy \\
\hline 224 & 20 & Very heavy \\
\hline
\end{tabular}




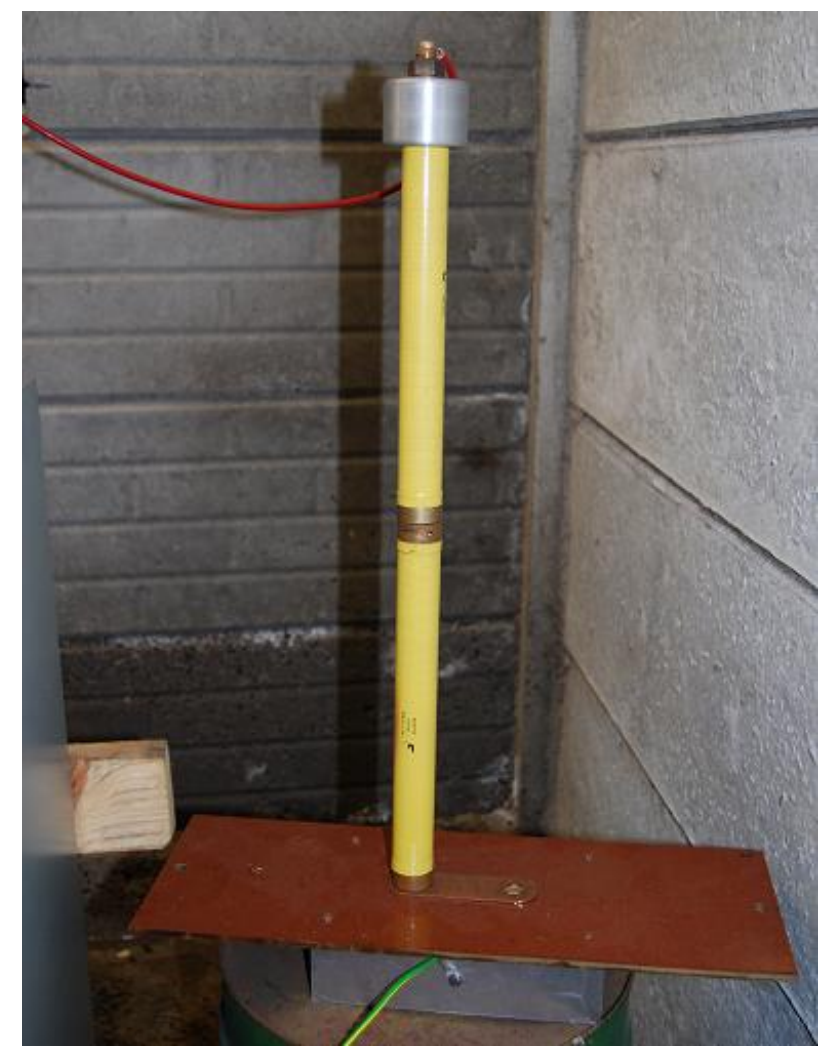

Figure D. 2: The voltage divider that was connected to the DC Source

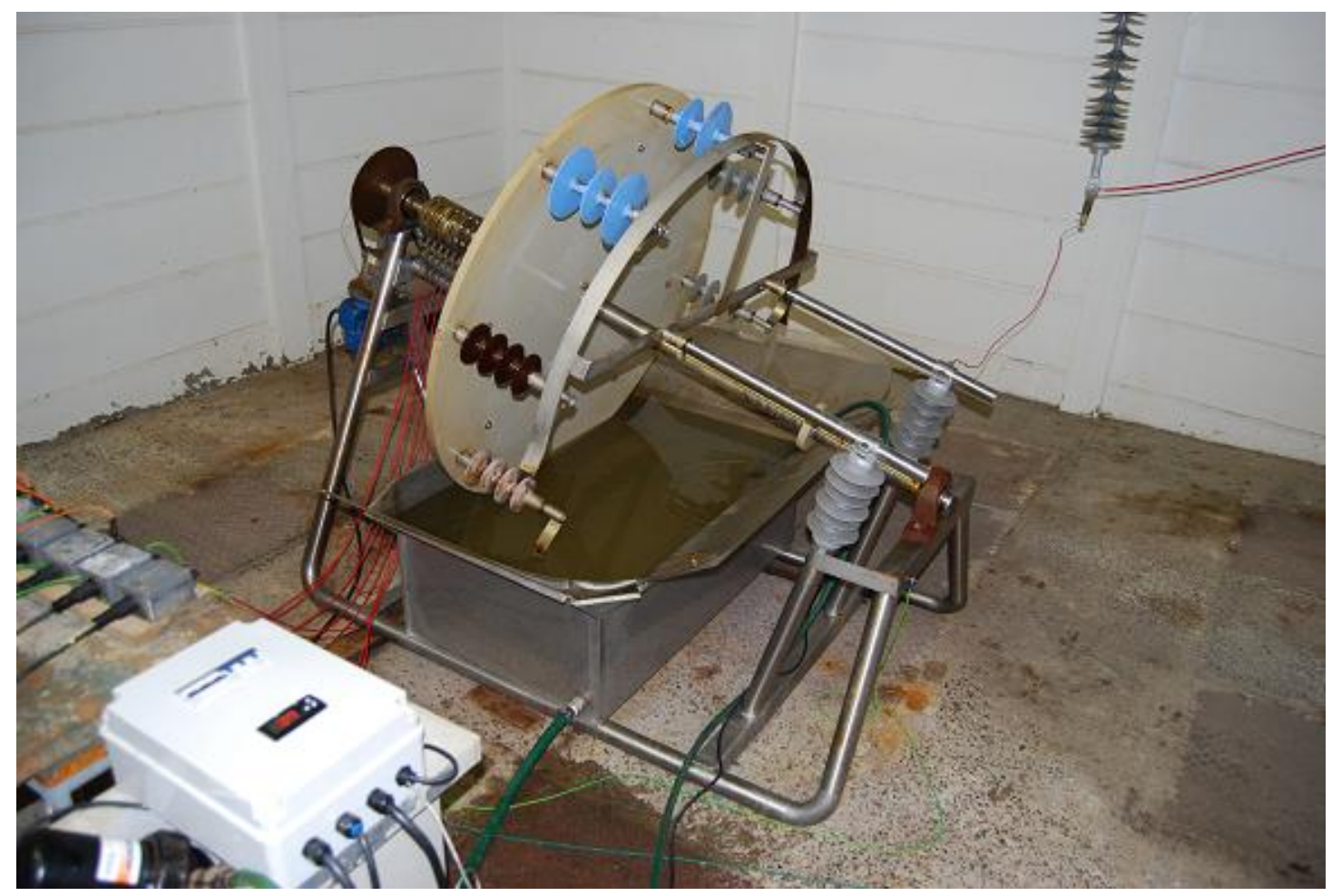

Figure D. 3: The Tracking Wheel Tester (TWT) with the fuse setup 